Universidade de São Paulo

Instituto de Física

\title{
Nanodispositivos Baseados em Grafeno
}

\author{
José Eduardo Padilha de Sousa
}

Orientador: Prof. Dr. Adalberto Fazzio 
"We can't solve problems by using the same kind of thinking we used when we created them."

Albert Einstein 


\section{Agradecimentos}

Gostaria de agradecer a todas as pessoas que me apoiaram nesta etapa importante da minha formação acadêmica. É impossível enumerar o nome de todas as pessoas que merecem os meus sinceros agradecimentos, mas fica a todos o meu muito obrigado. Em especial gostaria de agradecer:

Os meus pais, José Borges e Celeida, pelo amor incondicional e por todo apoio dedicado à minha formação.

A minha esposa, Ana Paula, por todo amor, carinho, dedicação, paciência e apoio dedicado em todo o meu doutorado.

Ao professor Adalberto Fazzio, pela incrível dedicação tanto na orientação de minha formação acadêmica quanto na minha formação pessoal.

Ao professor Antônio José Roque da Silva, por todas as discussões sobre física, que só fizeram aumentar o meu conhecimento.

A todos os colegas do grupo SAMPA, pelo incentivo, discussões, cafés e diversões.

Ao $\mathrm{CNPq}$ pelo apoio financeiro.

E principalmente a DEUS, que me deu muita saúde, força e dedicação. 


\section{Resumo}

Nesta tese investigamos a partir de cálculos de primeiros princípios, dispositivos e componentes de dispositivos baseados em grafeno. Abordamos os campos da nanoeletrônica e da spintrônica. Dentro da nanoeletrônica investigamos: (i) propriedades de transporte de um nanotransistor de bicamada de grafeno na presença de um gate duplo. Demonstramos que sobre a ação de um campo elétrico externo, mesmo utilizando um gate da ordem de $10 \mathrm{~nm}$, à temperatura ambiente e $4.5 \mathrm{~K}$ uma corrente nula nunca é exibida. Esses resultados são explicados por um regime de tunelamento; (ii) propriedades eletrônicas e de transporte de multicamadas de grafeno em função do número de camadas e tipo de empilhamento entre elas. Mostramos que a estrutura eletrônica do sistema depende fortemente desse novo grau de liberdade de empilhamento. Na presença de um campo elétrico externo aplicado perpendicular ao sistema, o empilhamento do tipo Bernal nunca exibe um gap de energia, ao contrário do empilhamento romboédrico que exige um gap ajustável através da intensidade do campo. Mostramos também que é possível diferenciar os tipos de empilhamentos através da resistência do sistema e variando-se a temperatura;(iii) dentro das componentes de um nanotransistor mais realista, estudamos as propriedades eletrônicas e estruturais de: (a) bicamadas de grafeno sobre um substrato de nitreto de boro hexagonal. Neste sistema o limite de voltagens que podem ser aplicadas depende fortemente do número de camadas de h-BN e da direção do campo, onde quanto menos camadas maior é a voltagem que pode ser aplicada; (b) heteroestruturas compostas de bicamadas de grafeno, nitreto de boro hexagonal e cobre. Demonstramos que para uma aplicação direta em um dispositivo a configuração com uma bicamada de grafeno depositada sobre um substrato de h-BN e este conjunto sobre a superfície de cobre é a mais favorável. Nessa configuração é possível tanto controlar o gap na bicamada como a dopagem do sistema, sem a abertura de canais de condução através do dielétrico (h-BN). Dentro do campo da spintrônica estudamos: (i) propriedades de transporte das nanofitas de grafeno (GNR) $(3,0)$ pristinas e dopadas com boro e nitrogênio. Para as GNR pristinas mostramos com os eletrodos em um alinhamento de spin anti-paralelo o sistema apresenta um comportamento de filtro de spin, onde para tensões de bias positivos/negativos somente o canal up/down conduz. Para as GNR dopadas com boro e nitrogênio, mostramos que as correntes para os diferentes canais de spin são não degeneradas ao longo de todo o intervalo de tensões aplicadas, apresentando desse modo um comportamento de filtro de spin; (ii) finalmente estudamos as propriedades de transporte de uma junção túnel magnética, composta de GNR intercaladas por uma nanofita de nitreto de boro hexagonal. Mostramos que esse sistema pode ser utilizado tanto como filtros de spin como elementos para dispositivos de magnetoresistência gigante, onde para este último a sua eficiência é muito mais pronunciada. 


\section{Abstract}

In this thesis we investigated by first principle calculations, devices and components of devices based on graphene. We covered the fields of nanoelectronics and spintronics. On the field of nanoelectronics we investigated: (i) the transport properties of a dual gate bilayer graphene nanotransistor. We showed that under the action of an external electrical field, even with a gate length of $10 \mathrm{~nm}$, at room temperature and $4.5 \mathrm{~K}$ a zero current is never exhibited. These results could be explained by a tunneling regime; (ii) the electronic and transport properties of few layer graphene, as function of the number and type of stacking of the layers. We show that the electronic structure strong deppends of the stacking order. On the presence of a external electrical field applied to the system, the Bernal stacking never presents a gap, contrary to the rombohedrical one, that posses a tuneable energy gap. Also we showed that is possible to differentiate the types of stacking by the resistance of the system and varying the temperature;(iii) for the components of a more realistic nanodevice, we study the structural and electronic properties of: (a) bilayer graphene over a hexagonal boron nitride substrate. We show that the voltages that could be applied to the system strongly depends of the number o layers and the direction of the field, where with more layers, smaller is the field; (b) heterostructures composed with bilayer graphene, hexagonal boron nitride and cooper. We show that for a direct application on a device, the better configuration is with a bilayer graphene over the hexagonal boron nitride, and this set over a cooper. In this configuration is possible to control both the gap and the doping of the system, without the creation of conducting channels through the dielectric (h-BN). On the field of spintronics, we study: (i) the transport properties $(3,0)$ graphene nanoribbons pristines and doped with nitrogen and boron. For the pristine GNR we show that for the electrodes in an anti-parallel alignment the system presents a spin filter behavior, where for positive/negative bias the transport is only by up/down channel. For the GNR doped with nitrogen and boron we show that the current is non-degenerated in all range of voltages applied, presenting a spin filter behavior; (ii) finally, we study the transport properties of a magnetic tunnel junction, consisting of a GNR intercalated with a hexagonal boron nitride nanoribbon. We show that such system could be used both as a spin filter as a device that uses the the giant magnetoresistance effect, where for the last the system if more efficient. 


\section{Sumário}

1 Introdução 1

2 Grafeno 5

3 Fundamentação Teórica 17

3.1 Teoria do Funcional da Densidade - DFT . . . . . . . . . . . . . . . 17

3.1 .1 Formulação do Problema . . . . . . . . . . . . . . . . . . . . . 17

3.1 .2 Teoremas de Hohenberg-Kohn . . . . . . . . . . . . . . . 18

3.1.3 Equações de Kohn-Sham . . . . . . . . . . . . . . . . . . . . . 19

3.2 Formalismo de Funções de Green: Transporte Eletrônico . . . . . . . . . . . . 21

3.2.1 Funções de Green no equilíbrio . . . . . . . . . . . . . . . . . . . . 22

3.2.2 Matriz densidade do sistema e grandezas físicas observáveis . . . . . . 26

3.2 .3 Matriz densidade no equilíbrio . . . . . . . . . . . . . . . . . 27

3.2.4 Matriz densidade fora do equilíbrio . . . . . . . . . . . . . . . . 29

3.2 .5 Análise e grandezas físicas . . . . . . . . . . . . . . . . . . 32

3.2.6 Representação esquemática do ciclo auto-consistente . . . . . . . . . . 33

3.3 Implementação de multigrid . . . . . . . . . . . . . . . . . 34

3.3.1 Detalhes da implementação de multigrid . . . . . . . . . . . . . 35

3.3.2 Testes da implementação de multigrid . . . . . . . . . . . . . . 36

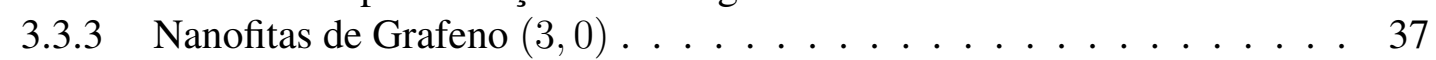

3.3.4 Grafeno e Bicamadas de Grafeno . . . . . . . . . . . . . . 38

4 Nanotransistor de bicamada de grafeno 41

4.1 Propriedades estruturais e eletrônicas da bicamada de grafeno . . . . . . . . . . 41

4.2 Transistor de bicamada de grafeno . . . . . . . . . . . . . . . . . 43

4.2.1 Procedimento de cálculo e setup utilizado . . . . . . . . . . . . . . . 45

4.2 .2 Resultados . . . . . . . . . . . . . . . . 47

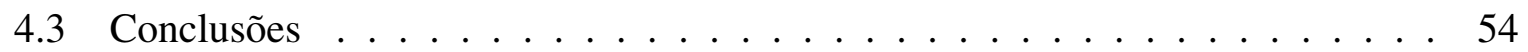

5 Propriedades Eletrônicas e de Transporte de Multicamadas de Grafeno 55

5.1 Energética dos possíveis empilhamentos das FLG . . . . . . . . . . 56

5.2 Importância do empilhamento na estrutura eletrônica do sistema . . . . . . . . 58

5.3 Influência do empilhamento nas propriedades de transporte das FLG . . . . . . 64

5.4 Conclusões . . . . . . . . . . . . . . . . . . . . . . 73 
6 Grafeno sobre substratos isolantes de h-BN $\quad 74$

6.1 O Nitreto de Boro Hexagonal - h-BN . . . . . . . . . . . . . . . . . . . 76

6.2 Bicamadas de Grafeno Sobre Substrato de Nitreto de Boro Hexagonal - BG+h-

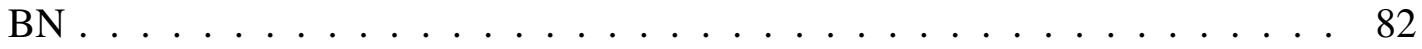

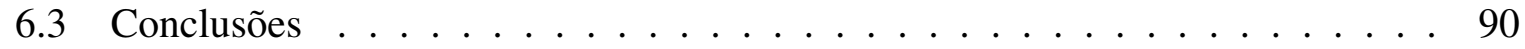

7 Interações entre canal e dielétrico com Cobre(111) 92

7.1 Bicamada de grafeno sobre $\mathrm{Cu}(111) \ldots \ldots \ldots$. . . . . . . . . . 95

7.2 Nitreto de Boro Hexagonal sobre $\mathrm{Cu}(111) \ldots \ldots . \ldots . \ldots . . \ldots 97$

7.3 Interações entre BG/Cobre(111)/h-BN . . . . . . . . . . . . . . . . . . . . 99

7.3.1 $\mathrm{Cu}(111) / \mathrm{h}-\mathrm{BN} /$ Bicamada . . . . . . . . . . . . . . 100

7.3.2 $\mathrm{Cu}(111) /$ Bicamada/h-BN . . . . . . . . . . . . . . . . . 102

7.3.3 $\mathrm{Cu}(111) / \mathrm{hBN} /$ Bicamada/hBN . . . . . . . . . . . . 103

7.4 Conclusões . . . . . . . . . . . . . . . . . . . 105

8 Propriedades de transporte de nanofitas de grafeno: pristinas e dopadas com boro

$\begin{array}{ll}\text { e nitrogênio } & 108\end{array}$

8.1 Metodologia . . . . . . . . . . . . . . . . . . . 109

8.1.1 Transporte dependente do spin . . . . . . . . . . . . . . 113

8.2 Resultados e Discussões . . . . . . . . . . . . . . . . . . . . . . . . 114

8.3 Nanofita de grafeno $(3,0)$ pristina . . . . . . . . . . . . . . . . 114

8.4 Dopagem da nanofita com boro e nitrogênio . . . . . . . . . . . . . . 117

8.5 Junção túnel magnética . . . . . . . . . . . . . . . . . . . . . . 122

8.6 Conclusões . . . . . . . . . . . . . . . . . . . . . 128

9 Conclusões 131

$\begin{array}{ll}\text { A Multigrid } & 134\end{array}$

A.1 Discretização do Problema . . . . . . . . . . . . . . . . . . . . . . . . 134

A.1.1 Problema Modelo 1 - Caso Unidimensional . . . . . . . . . . . . . . 134

A.1.2 Problema Modelo 2 - Caso Bidimensional . . . . . . . . . . . . . . . 137

A.2 Métodos Iterativos para Sistemas Lineares . . . . . . . . . . . . 138

A.2.1 Erro e Resíduo . . . . . . . . . . . . . . . . . . . . . . . 138

A.2.2 Método de Jacobi . . . . . . . . . . . . . . . . . . . . . . . . 139

A.2.3 Iteração de Gauss-Seidel . . . . . . . . . . . . . . . . . . . . 143

A.3 Exemplos da atuação dos métodos iterativos - Jacobi e Gauss-Seidel . . . . . 145

A.4 Observações antes de entrar no método de multigrid . . . . . . . . . . . . 147

A.5 Operadores de transferência entre grids . . . . . . . . . . . . . . 150

A.5.1 Operadores de interpolação . . . . . . . . . . . . . . . . . . 150

A.5.2 Operadores de restrição . . . . . . . . . . . . . . . . . . . . . . . . 152

A.6 Esquema de correção de dois grids $-\mathbf{v}^{h} \leftarrow M G\left(\mathbf{v}^{h}, \mathbf{f}^{h}\right) \ldots \ldots \ldots$

A.7 Ciclos Multigrid . . . . . . . . . . . . . . . . . . 155

A.7.1 Ciclo $V-\mathbf{v}^{h} \leftarrow V^{h}\left(\mathbf{v}^{h}, \mathbf{f}^{h}\right) \ldots \ldots \ldots \ldots 155$

A.7.2 Ciclo full-multigrid $-\mathrm{v}^{h} \leftarrow F M G^{h}\left(f^{h}\right) \ldots \ldots \ldots 156$

$\begin{array}{lll}B & \text { Lista de Publicações } & 158\end{array}$ 
Referências Bibliográficas

159 


\section{Lista de Figuras}

2.1 Formas alotrópicas do carbono. . . . . . . . . . . . . 6

2.2 Grafeno, um cristal de uma única camada: o mais fino material já isolado na natureza. (a) Imagem de microscopia de força atômica de um grafeno. (b) Uma única folha de grafeno suspensa livremente entre contatos metálicos. (c) Imagem de microscopia eletrônica de varredura de um único cristal de grafeno. Esta imagem mostra que a maioria das bordas desse sistema possui uma orientação zig-zag e arm-chair, indicada pelas linhas em azul e vermelho, respectivamente. Imagem retirada da referência [1] . . . . . . . . . . . . .

2.3 (a) Rede hexagonal do grafeno com dois átomos por célula unitária, A e B. (b) Estrutura de bandas $3 D$ do grafeno. (c) Estrutura de bandas do grafeno. (d) Aproximação de baixas energias para a estrutura de bandas do grafeno, onde dois cones tocam-se no ponto de Dirac. A posição do nível de Fermi em relação ao ponto de Dirac determina o tipo de dopagem no grafeno. Imagem retirada na referência [2]. . . . . . . . . . . . . . . . . . . .

2.4 (a) Condutância de uma única camada de grafeno utilizada como canal em um transistor de efeito de campo, em função da voltagem de top gate, para diferentes voltagens de back gate. $\mathrm{O}$ inset mostra esquematicamente o modelo do dispositivo utilizado. Imagem retirada da referência [3]. (b) Mobilidades para elétrons e buracos de uma única camada de grafeno exfoliada e incorporada como canal de um transistor com um substrato de $\mathrm{SiO}_{2}$. Imagem retirada da

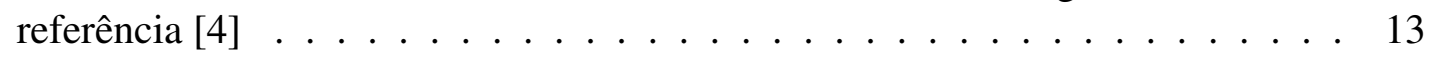

2.5 Visão esquemática da bicamada de grafeno com empilhamento de Bernal AB. . 15

2.6 (a) Bandas de energia para uma bicamada de grafeno. Linha tracejada/cheia corresponde ao sistema na ausência/presença de campo elétrico aplicado. Imagem retirada da referência [5]. (b) Comportamento da corrente fonte-dreno para um transistor de bicamada de grafeno, em função da voltagem de top-gate.

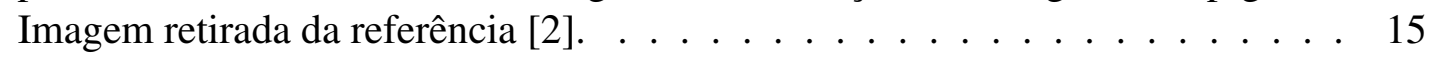

3.1 Esquema das componentes do sistema utilizado nos cálculos de transporte. . . . 22

3.2 (a) Configuração do sistema tratado pelo método de funções de Green fora do equilíbrio (NEGF), contendo os eletrodos semi-infinitos esquerdo (L) e direito (R) ao longo do eixo $a$. Esses eletrodos podem possuir condições periódicas de contorno no plano bc. (b) Sistema unidimensional compactado a partir da configuração (a) utilizando a condição de periodicidade no plano $b c$, onde a região $C$ é a região central estendida consistindo de $C_{0}, L_{0}$ e $R_{0} \ldots \ldots 23$

3.3 Contorno fechado para integração no equilíbrio. Os termos $\gamma, \theta, R C, C C, C L$ são parâmetros utilizados no método de integração de Gauss-Legengre [57]. . . 
3.4 Representação esquemática das condições de contorno para os cálculos fora do equilíbrio. (a-1) potencial de Hartree médio para o sistema pristino. (a-2) Potencial de Hartree do sistema (a-1) sujeita à rampa de potencial. (b) Esquema para as condições de contorno no cálculo multigrid. . . . . . . . . . . . . 30

3.5 Contorno fechado para integração fora do equilíbrio. Os termos $\gamma, \theta, R C, C C$, $C L$ são parâmetros utilizados no método de integração de Gauss-Legendre [57]. 31

3.6 Ciclo auto-consistente do código TRANSAMPA2.0 . . . . . . . . . . . . . 34

3.7 Estrutura de bandas e densidade de estados para a nanofita zig-zag $(3,0)$ nas configurações:(a) Acoplamento anti-ferromagnético entre as bordas e (b) Acoplamento ferromagnético. As linhas cheias são cálculos com o SIESTA-FFT e as pontilhadas com a implementação do Multigrid. . . . . . . . . . . . . . . . . .

3.8 Densidade de estados e estrutura de bandas para a monocamada de grafeno. As linhas cheias são cálculos com o SIESTA-FFT e as pontilhadas com a implementação do Multigrid. . . . . . . . . . . . . . . . . . . . . .

3.9 Densidade de estados e estrutura de bandas para a bicamada de grafeno. As linhas cheias são cálculos com o SIESTA-FFT e as pontilhadas com a implementação do Multigrid. . . . . . . . . . . . . . . . . . . . . . . .

3.10 (a) Representação esquemática da bicamada de grafeno na presença do campo elétrico e/ou gate aplicado. O campo é aplicado no sistema inteiro, onde este é periódico em todas as direções. Estrutura de bandas para a bicamada na presença de um campo elétrico de intensidade (a) $0.1 \mathrm{~V} / \AA ̊$ e (b) $0.2 \mathrm{~V} / \AA$, calculadas com o SIESTA-FFT (linhas pretas contínuas) e SIESTA-MG (linhas pontilhadas vermelhas $) \ldots \ldots \ldots$. . . . . . . . . . . . 40

4.1 Visão esquemática da bicamada de grafeno com empilhamento de Bernal AB. . 42

4.2 Estrutura de bandas para a bicamada de grafeno com as aproximações (a) LDA e (b) GGA+vdW.

4.3 (a)Estrutura de bandas para uma bicamada de grafeno na presença de um campo elétrico de $5 \mathrm{~V} / \mathrm{nm}$, mostrando o gap no ponto $K, \Delta_{K}$, e o gap homo-lumo, $\Delta_{g}$. (b)Gap de energia em função do campo aplicado para a BG. . . . . . . . . . . .

4.4 (a)Micrografia eletrônica de varredura, colorida artificialmente, de uma bicamada de grafeno suspensa entre eletrodos de uma liga de $\mathrm{Cr} / \mathrm{Au}$ e na presença de um top gate. Imagem retirada da referência [69]. (b)Imagem de microscopia ótica de uma bicamada de grafeno suportada sobre um substrato de $\mathrm{SiO}_{2}$, entre dois eletrodos de ouro e na presença de um eletrodo de top gate. Imagem retirada da referência $[7] \ldots \ldots \ldots \ldots$

4.5 Visão esquemática do nanotransistor de bicamada de grafeno com duplo gate que consideramos em nossas simulações. $V_{d s}, V_{b g}, V_{t g}, L_{s c a t t}, L_{\text {gate }}$ correspondem a voltagem fonte-dreno, voltagem de back-gate, voltagem de top-gate, comprimento da região de espalhamento e comprimento do gate, respectivamente. 45

4.6 Seção longitudinal da região do sistema na presença dos potenciais de back/top gate. A imagem mostra a diferença entre o potencial eletrostático de um sistema na presença de campo e o sistema sem campo. . . . . . . . . . . . . .

4.7 Em (a) apresentamos as curvas $I_{d s} \times V_{d s}$ variando $\Delta V$, para $300 K$ e $L_{\text {gate }}=$ $5 \mathrm{~nm}$. Em (b) apresentamos as curvas $I_{d s} \times V_{d s}$ variando $L_{\text {gate }}$ para $300 \mathrm{~K}$ e

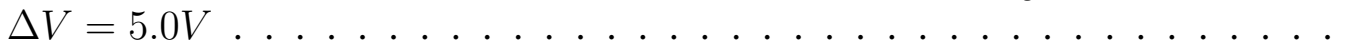


4.8 Características do transporte eletrônico de um FET de bicamada de grafeno com duplo gate, para $L_{\text {gate }}=5 \mathrm{~nm}$ e $V_{b g}=+2.5 \mathrm{~V}$. A corrente (quadrados azuis) e carga induzida (círculos pretos) à (a) Temperatura ambiente e (b) $\mathrm{T}=4.5 \mathrm{~K} .49$

$4.9 I_{d s}$ em função de $V_{t g}$ variando-se $L_{\text {gate }}$ à temperatura (a) $300 \mathrm{~K}$ e (b) $4.5 \mathrm{~K}$. A voltagem fonte-dreno foi mantida em $10 \mathrm{mV}$ e $V_{b g}=+2.5 \mathrm{~V} \ldots \ldots \ldots$. . . . 50

4.10 (a) Transmitâncias para $L_{\text {gate }}=2 n \mathrm{~m}$, variando-se $V_{t g}$. Inset(a) mostra a diferença das funções de Fermi-Dirac dos eletrodos para $4.5 \mathrm{~K}$ e $300 \mathrm{~K}$ com uma tensão de bias de $10 \mathrm{mV}$. (b) Transmitância para $L_{\text {gate }}=10 \mathrm{~nm}$, variando-se $V_{t g}$. Inset(b) Detalhe da transmitância para $L_{\text {gate }}=10 \mathrm{~nm}$ no ponto de neutralidade de carga, em uma região ao redor do nível de Fermi. . . . . . . . . . . . .

4.11 (a) Diferença local entre as cargas de um sistema sem gate com um sistema na presença de um gate. Como pode ser visto, existe uma transferência de elétrons a partir da camada superior para a inferior(Unidades em $e / b o h r^{3}$ ). (b) Densidade de estados projetada a cada quatro átomos (equivalentes à célula unitária da BG) ao longo da direção z. O sistema considerado aqui possui $L_{\text {gate }}=10 \mathrm{~nm}$ e está no ponto de neutralidade de carga. . . . . . . . . . . 52

4.12 Curvas $I_{d s} \times L_{\text {gate }}$ para $\Delta V=5.0 \mathrm{~V}$ e $V_{d s}=10 \mathrm{mV}$, para $300 \mathrm{~K}$ e $4.5 \mathrm{~K}$. Para todos os gráficos, $\Delta V=2\left|V_{t g}\right|$, com $V_{b g}=-V_{t g} \ldots \ldots \ldots \ldots$

5.1 Representação esquemática dos empilhamentos Bernal ( $\mathrm{AB}$ ) e Romboédrico (ABC) para: (a-1) Tricamadas AB; (b-1) Tetracamadas AB; (c-1) Pentacamadas AB; (a-2) Tricamdas ABC; (b-2) Tetracamadas ABC; (c-2) Pentacamadas ABC. . . . . . . . . . . . . . . . . . . . .

5.2 Estrutura de bandas para as tricamadas $\mathrm{ABA}$ e $\mathrm{ABC}$; tetracamadas $\mathrm{ABAB}$ e ABCA; e pentacamadas ABABA e ABCAB; utilizando (a) LDA e (b) GGA+vdW. 59

5.3 Estruturas de bandas para as FLG na presença de um campo elétrico externo de $3 \mathrm{~V} / \mathrm{nm}$ para as: Tricamadas (a) ABA e (b) ABC; Tetracamadas (c) ABAA e (d) ABCA; Pentacamadas (e) ABABA e (f) ABCAB. Nos insets de cada figura, temos a estrutura de bandas para cada sistema sem a presença de campo. . . . .

5.4 Estrutura de bandas para as FLG no empilhamento de Bernal perto do ponto K. Em: (a) Tetracamada - campo elétrico nulo; (b) Tetracamada - campo elétrico $0.3 \mathrm{~V} / \mathrm{nm}$; (c) Esquema de divisão dos subsistemas da tetracamada. Em: (d) Pentacamada - campo elétrico nulo; (e) Pentacamada - campo elétrico $0.3 \mathrm{~V} / \mathrm{nm}$; (f) Esquema de divisão dos subsistemas da pentacamada. . . . . . . . . . . . . .

5.5 Estrutura de bandas para as FLG no empilhamento romboédrico perto do ponto K. Em: (a) Tetracamada - campo elétrico nulo; (b) Tetracamada - campo elétrico $0.3 \mathrm{~V} / \mathrm{nm}$; (c) Esquema de divisão dos subsistemas da tetracamada. Em: (d) Pentacamada - campo elétrico nulo; (e) Pentacamada - campo elétrico $0.3 \mathrm{~V} / \mathrm{nm}$; (f) Esquema de divisão dos subsistemas da pentacamada. . . . . . . . . . . . 63

5.6 Gap de energia em função do campo elétrico externo para as bicamadas, tricamadas, tetracamadas e pentacamadas, no empilhamento ABC. . . . . . . . . . 64

5.7 Representação esquemática do sistema utilizado nos cálculos de transporte das FLG. . . . . . . . . . . . . . . . . . . . 65 
5.8 Estrutura de bandas, densidade de estados (DOS), transmitância $(T(E)$ ) e densidade local de estados (LDOS), para as FLG: (a) Tricamada; (b) Tetracamada; (c) Pentacamada; (d) Hexacamada. Nos quadros superiores em cada figura temos o empilhamento de Bernal e nos quadros inferiores o empilhamento romboédrico.

5.9 (a) Estrutura de bandas para hexacamada no empilhamento romboédrico mostrando os intervalos de integração para a densidade local de estados. (b) Densidade local de estados para $0.2 \mathrm{eV}$ de integração ao redor do nível de Fermi. (c) Densidade local de estados para $0.6 \mathrm{eV}$ de integração ao redor do nível de Fermi. 67

5.10 Densidade local de estados - LDOS - para (a) tricamada; (b) tetracamada; (c) pentacamada. O intervalo de integração foi de $0.2 \mathrm{eV}$ ao redor do nível de Fermi. 68

5.11 Correntes fonte-dreno para as FLG de 1 até 12 camadas para as FLG em ambos os empilhamentos, respectivamente. . . . . . . . . . . . . . . 68

5.12 (a) Resistência para as FLG em ambos os empilhamentos e em função do número de camadas de grafeno. (b) Resistência para as FLG em ambos os empilhamentos em função do inverso do número de camadas. . . . . . . . . . . 70

5.13 Corrente em função da temperatura para os empilhamento de Bernal e romboédrico, de 3 até 12 camadas. . . . . . . . . . . . . . . .

5.14 (a,b) Comportamento da estrutura de bandas para o caso romboédrico em função do número de camadas no sistema. (c) Comportamento da distância entre os estados do interior da FLG em função do número de camadas. . . . . . . . . . . .

6.1 Comparação entre a topografia e densidades de carga para grafeno sobre h-BN versus grafeno sobre $\mathrm{SiO}_{2}$.(a,b) Imagens das topografia obtidas por STM para (a) Grafeno/h-BN e (b) Grafeno/ $\mathrm{SiO}_{2}$. (c,d) Imagens das densidades de carga (a) Grafeno/h-BN e (b) Grafeno/SiO $\mathrm{S}_{2}$. Nestas iamgens é possível observar a inhomogeneidade da topografia e da densidade de carga no grafeno sobre o $\mathrm{SiO}_{2}$, e como esse problema é praticamente inexistente sobre o substrato de nitreto de boro hexagonal. Figura retirada da referência [100]. . . . . . . . . .

6.2 (a) Geometria de uma única camada de h-BN, mostrando os átomos de boro e nitrogêncio ocupando as subredes A e B da estrutura hexagonal. (b) Empilhamento $A A^{\prime}$ das monocamadas de h-BN (estrutura encontrada na natureza). (c) Nitreto de Boro Hexagonal vendido comercialmente (conhecido como grafite braco) . . . . . . . . . . . . . . . . . . . 76

6.3 Estrutura de bandas para o Nitreto de Boro Hexagonal utilizando o parâmetro de rede do (a) h-BN e (b) do grafeno. . . . . . . . . . . . . . . . . . . .

6.4 Estrutura de bandas para o Nitreto de Boro Hexagonal para 2 h-BN - (a1) sem campo e (a2) com campo de $2 \mathrm{~V} / \mathrm{nm}$, e $5 \mathrm{~h}$-BN - (b1) sem campo e (b2) com campo de $2 \mathrm{~V} / \mathrm{nm}$.

6.5 (a) Média planar da densidade eletrônica induzida para cinco camadas de h-BN na presença de um campo de $1 \mathrm{~V} / \mathrm{nm}$. (b) Constante dielétrica média em função do campo elétrico externo para 2, 3, 4 e 6 camadas de h-BN. (c) Constante dielétrica média em função do número de camadas de h-BN para um campo elétrico de $1 \mathrm{~V} / \mathrm{nm}$.

6.6 Representação estrutural dos possíveis empilhamentos da bicamada de grafeno em relação ao substrato de h-BN. . . . . . . . . . . . . . . . . . . . 
6.7 Energia total em função da distância $d$ de separação entre a camada superior de h-BN com a camada inferior para (a) grafeno e (b) bicamada de grafeno, nos empilhamentos mostrados na figura 6.6. Os valores das energias são dados em relação à configuração com menor energia para cada sistema. Essas curvas foram construídas utilizando a aproximação LDA para o termo de troca e correlação. . . . . . . . . . . . . . . . . . . . . .

6.8 Estrutura de bandas para as bicamadas de grafeno sobre h-BN(AA'), em função do número de camadas de h-BN para: (a) 1 h-BN; (b) 5 h-BN; (c) 12 h-BN e (d) $18 \mathrm{~h}-\mathrm{BN}$, na presença de um campo elétrico perpendicular, variando de $+1 \mathrm{~V} / \mathrm{nm}$ a $+5 \mathrm{~V} / \mathrm{nm}$ painéis superiores e $-1.0 \mathrm{~V} / \mathrm{nm}$ a $-5.0 \mathrm{~V} / \mathrm{nm}$ painéis inferiores. O nível de Fermi do sistema está no zero. . . . . . . . . . . . . . .

6.9 (a) Diferença entre as densidades de carga na presença de um campo elétrico de $+2 \mathrm{~V} / \mathrm{nm}$ e sem campo, $\Delta \rho=\rho_{E}-\rho_{0}$. Vermelho indica $\Delta \rho$ positivo e amarelo indica $\Delta \rho$ negativo. $\mathrm{O}$ valor da isosuperfície utilizada foi de $1 \times 10^{-4} \mathrm{e} / \mathrm{bohr}$. (b) Visão esquemática dos gaps de energia $\Delta K$ e $\Delta g$, na bicamada de grafeno. (c) Gaps de energia nas bicamadas de grafeno em função do número de camadas de h-BN e do campo elétrico aplicado. . . . . . . . . . . . . . .

6.10 Densidade de estados projetada para uma bicamada de grafeno sobre 5 camadas de nitreto de boro hexagonal, (a) sem campo elétrico, (b) com um campo elétrico positivo de $+4.0 \mathrm{~V} / \mathrm{nm}$ e (c) com um campo elétrico negativo de $-4.0 \mathrm{~V} / \mathrm{nm}$. As linhas pontilhadas em (b) e (c) representam a região próxima ao nível de Fermi onde utilizamos para plotar a LDOS (d,e). O nível de Fermi do sistema foi definido como sendo zero na figura. . . . . . . . . . . . . . . 88

6.11 Tipos de empilhamentos possíveis para o cristal de h-BN . . . . . . . . . . . . 89

6.12 Estruturas de bandas para a bicamada de grafeno sobre h-BN. Apresentamos os seis possíveis empilhamentos na presença de um campo elétrico aplicado de (a) $+2 V / n m$ e (b) $-2 V / n m \ldots \ldots \ldots \ldots \ldots$. . . . . . . . . . . . . 90

6.13 (a) Visão esquemática da bicamada de grafeno sobre h-BN no empilhamento $A B / A B / A B$, para 5 e 12 camadas; (b) Estrutura de bandas para os sistemas em (a), em função do campo elétrico aplicado, variando de $\pm 1 \mathrm{~V} / \mathrm{nm}$ até $\pm 5 \mathrm{~V} / \mathrm{nm}$. Como verificado para o empilhamento $A A^{\prime}$, quanto menos camadas presente, maior fica a intensidade do campo elétrico necessário para criar um canal de condução através do substrato.

7.1 Fotografias do processo de fabricação de filmes de grafeno em larga escala. Imagem retirada da referência $[118] . \ldots \ldots \ldots \ldots$

7.2 (a) Representação esquemática de um nanotransistor de bicamada de grafeno, onde são utilizados o nitreto de boro hexagonal como substrato e dielétrico e os eletrodos do sistema são constituídos por cobre. (b,c,d) Representação esquemática de possíveis configurações entre cobre/h-BN/BG que podem ser encontradas em um nanodispositivo. Estas seções serão estudadas separadamente. 94

7.3 Empilhamentos utilizados nos cálculos das bicamadas de grafeno sobre a superfície (111) do Cobre. Para este sistema existem 3 possíveis configurações :(a) átomo de cobre no meio da ligação entre dois átomos de carbono; (b) átomo de cobre no meio do hexágono da camada de grafeno inferior; (c) átomo de cobre abaixo de um átomo de carbono da camada inferior. . . . . . . . . . . . . . . . 
7.4 Curvas de energia potencial para os três possíveis empilhamentos das bicamadas de grafeno sobre a superfície (111) do cobre. . . . . . . . . . . . . . 96

7.5 Evolução da estrutura de bandas de uma bicamada de grafeno sobre cobre(111). Estruturas de bandas para o sistema (a,d) sem campo elétrico externo e com campo elétrico externo aplicado de (b) $+2.0 \mathrm{~V} / \mathrm{nm}$, (c) $+5.0 \mathrm{~V} / \mathrm{nm}$, (e) $-2.0 \mathrm{~V} / \mathrm{nm}$ e (f) $-5.0 \mathrm{~V} / \mathrm{nm} . \ldots \ldots \ldots \ldots \ldots$. . . . . . . . . . . . . . . . 97

7.6 Gap de energia induzido na bicamada de grafeno. Em (a) $\Delta_{g}$, menor distância entre as bandas das bicamadas; (b) $\Delta_{K}$, gap no ponto de alta simetria $K$ do grafeno. . . . . . . . . . . . . . . . . .

7.7 Empilhamentos utilizados nos cálculos do h-BN sobre a superfície (111) do Cobre. (a) Centro do hexágono do h-BN sobre um átomo de cobre; (b) átomo de nitrogênio sobre um átomo de cobre; (c) átomo de boro sobre um átomo de cobre; (d) vista lateral da estrutura contendo uma única camada de h-BN depositada sobre uma superfície de cobre com 7 planos atômicos de cobre. . . . 98

7.8 Curvas de energia potencial para os três possíveis empilhamentos do h-BN sobre a superfície (111) do cobre. . . . . . . . . . . . . . . . . . . . . . . . 99

7.9 Evolução da estrutura de bandas para o h-BN sobre cobre(111) para (a) 1 camada de h-BN; (b) 2 camadas de h-BN; (c) 3 camadas de h-BN; (d) 4 camadas de h-BN. Em todas as figuras apresentamos os resultados para o sistema na ausência de campo elétrico externo e na presença de campo - quadros superiores campos positivos e quadros inferiores campos negativos. . . . . . . . . . 100

7.10 Representação das geometrias utilizadas. (a) 1 camada de h-BN; (b) 2 camadas de h-BN (c) 3 camadas de h-BN; (d) 4 camadas de h-BN. . . . . . . . . . . . . 101

7.11 Evolução da estrutura de bandas para o sistema $\mathrm{Cu}(111) / \mathrm{h}-\mathrm{BN} / \mathrm{BG}$ para: (a) 1 camada de h-BN; (b) 2 camadas de h-BN; (c) 3 camadas de h-BN; (d) 4 camadas de h-BN. Em todas as figuras apresentamos os resultados para o sistema na ausência de campo elétrico externo e na presença de campo - quadros superiores campos positivos e quadros inferiores campos negativos. . . . . . 102

7.12 Gap de energia induzido na bicamada de grafeno em função do campo elétrico externo para: (a) $\mathrm{Cu}(111) / 1 \mathrm{~h}-\mathrm{BN} / \mathrm{BG}$; (b) $\mathrm{Cu}(111) / 2 \mathrm{~h}-\mathrm{BN} / \mathrm{BG}$; (c) $\mathrm{Cu}(111) / 3 \mathrm{~h}-$ BN/BG; (d) $\mathrm{Cu}(111) / 4 h-B N / B G$. Este gap é calculado como sendo a menor distância entre as bandas das bicamadas de grafeno. . . . . . . . . . . . . . 103

7.13 Representação das geometrias utilizadas. (a) 1 camada de h-BN; (b) 2 camadas de h-BN; (c) 3 camadas de h-BN; (d) 4 camadas de h-BN. . . . . . . . . . . . 104

7.14 Evolução da estrutura de bandas para o sistema $\mathrm{Cu}(111) / \mathrm{BG} / \mathrm{h}-\mathrm{BN}$ para: (a) 1 camada de h-BN; (b) 2 camadas de h-BN; (c) 3 camadas de h-BN; (d) 4 camadas de h-BN. Em todas as figuras apresentamos os resultados para o sistema na ausência de campo elétrico externo e na presença de campo - quadros superiores campos positivos e quadros inferiores campos negativos. . . . . . 105

7.15 Evolução da estrutura de bandas para o sistema $\mathrm{Cu}(111) / \mathrm{h}-\mathrm{BN} / \mathrm{BG} / \mathrm{h}-\mathrm{BN}$ em função do campo elétrico aplicado para: (a) 1 camada de h-BN; (b) 3 camadas de h-BN. Em todas as figuras apresentamos os resultados para o sistema na ausência de campo elétrico externo e na presença de campo - quadros superiores campos positivos e quadros inferiores campos negativos. . . . . . . . . 106 
8.1 (a)Representação esquemática do sistema utilizado nos cálculos de transporte eletrônico. O dispositivo é composto pelos eletrodos direito(R) e esquerdo(L) e uma região de espalhamento $(\mathrm{C})$, que é conectada aos eletrodos pelas regiões de buffer direita e esquerda. O átomo em verde na figura marca a configuração mais estável para os átomos de boro e nitrogênio utilizados na dopagem das nanofitas. (b) Represetação do sistema em termos das matrizes Hamiltonianas de cada bloco utilizado nos cálculos. . . . . . . . . . . . . . . . . . . . . . 110

8.2 Diferença local entre as densidades de carga para os spins up e down, $\rho_{u p}$ $\rho_{\text {down }}$, para a nanofita de grafeno $(3,0)$ nas configurações: (a) Ferromagnética (FM) e (b) Antiferromagnética (AFM). As cores laranja e vermelha correspondem a spin up e down, respectivamente. $\mathrm{O}$ valor da isosuperfície utilizada foi de $0.002 e / b o h r^{3}$

8.3 (a) Curvas $I \times V$ dependentes do spin para uma nanofita de grafeno $(3,0)$ com alinhamento FM dos eletrodos. A curva preta e vermelha correspondem aos spins up e down, respectivamente. (b), (c) e (d) Densidade de estados projetada nos átomos de carbono das bordas e na região de bulk das nanofitas e, curvas de transmitância para diferentes voltages de bias: $+0.1,+0.2 \mathrm{e}+1.0 \mathrm{~V}$, respectivamente. . . . . . . . . . . . . . . .

8.4 (a) Painel superior: Transmitância e função de distribuição de Fermi-Dirac em função da energia para os spins up e down. Painel inferior: Produto da transmitância com a diferença das funções de Fermi-Dirac para os eletrodos esquerdo e direto, para uma voltagem de bias de $+1.0 \mathrm{~V}$. (b) Painel superior: Transmitância e função de distribuição de Fermi-Dirac em função da energia para os spins up e down. Painel inferior: Produto da transmitância com a diferença das funções de Fermi-Dirac para os eletrodos esquerdo e direto, para uma voltagem de bias de $+0.2 V \ldots \ldots \ldots \ldots \ldots . \ldots . \ldots . \ldots$

8.5 (a) Curvas $I \times V$ dependentes do spin para a nanofita de grafeno na configuração dos eletrodos AFM. As curvas em preto e azul são para as correntes up e down, respectivamente. Densidade de estados projetada nos átomos de carbono de borda e bulk, e transmitâncias para diferentes voltagens de bias: (b) $0.0 \mathrm{~V}$; (c) $+0.3 V$; e $(\mathrm{d})+1.1 V \ldots \ldots \ldots \ldots \ldots \ldots$

8.6 Transmitâncias para os canais de spin up e down, em função da voltagem de bias para a configuração AFM dos eletrodos. . . . . . . . . . . . . . . . . 118

8.7 Representação esquemática das nanofitas dopadas com boro e nitrogênio. . . . . 119

8.8 Diferença local entre as densidades de carga para os spins up e down, $\rho_{u p}-$ $\rho_{\text {down }}$, para a nanofita de grafeno $(3,0)$ dopadas com (a) boro e (b) nitrogênio. As cores laranja e vermelha correspondem a spin up e down, respectivamente. $\mathrm{O}$ valor utilizado para a isosuperfície de $0.002 e / b o h r^{3}$.

8.9 (a) Curvas $I \times V$ dependentes do spin para a nanofita de grafeno dopada com boro. As curvas em azul e vermelho são para as correntes com spin up e down, respectivamente. Densidade de estados projetada e curvas de transmitância para diferentes voltagens de bias: (b) $0.0 \mathrm{~V}$; (c) $+1.0 \mathrm{~V}$; e (d) $+1.5 \mathrm{~V} \ldots \ldots$. . . 121

8.10 (a) Curvas $I \times V$ dependentes do spin para a nanofita de grafeno dopada com nitrogênio. As curvas em azul e vermelho são para as correntes com spin up e down, respectivamente. Densidade de estados projetada e curvas de transmitância para diferentes voltagens de bias: (b) $0.0 \mathrm{~V}$; (c) $+0.5 \mathrm{~V}$; e (d) $+1.5 \mathrm{~V} . .122$ 
8.11 (a)Representação esquemática da geometria utilizada nos cálculos de transporte eletrônico. Os sistemas utilizados nos cálculos de transporte correspondem a $N$ uc (célula unitária), com $N(N=1,2, \cdots, 6)$. (b) Representação esquemática da configuração magnética dos eletrodos. Os cálculos foram realizados com um alinhamento paralelo (P) e antiparalelo (AP) dos eletrodos. . . .

8.12 Diferença local entre as densidades de carga para os spins up e down, $\rho_{u p}-$ $\rho_{\text {down }}$, para a nanofita de grafeno $(3,0)$ para os sistemas com: (a) 3 uc de $B N$ com alinhamento paralelo dos eletrodos; (b) 3 uc de $B N$ com alinhamento antiparalelo dos eletrodos. As cores vermelha e amarela correspondem à spin up e down, respectivamente. O valor utilizado para a isosuperfície foi de $0.001 e / b o h r^{3}$.

8.13 Transmitâncias para os sistemas considerados. (a)Curvas tracejadas correspondem ao sistema pristino e as curvas cheias ao sistema com 1uc de BN; (b) 2 uc de BN; (c) 3 uc de BN; (d) 4 uc de BN; (e) 5 uc de BN; (f) 6 uc de BN. Em cada gráfico apresentamos um inset, ampliando uma pequena região ao redor do nível de Fermi, para evidenciar o valor finito da transmitância. . . . . . . . . . 125

8.14 (a) Corrente em função da voltagem de bias para a nanofita de grafeno pristina com alinhamento paralelo dos eletrodos. (b) Polarização da corrente em função da voltagem de bias para a nanofita pristina. . . . . . . . . . . . . 126

8.15 Corrente em função da tensão de bias para: (a) 1, (b) 2, (c) 3, (d) 4, (e) 5 e (f) 6 células unitárias de BN. O eixo da corrente está em escala log para uma melhor visualização.

8.16 Polarização da corrente em função da voltagem de bias, $V_{d s}$, para $N$ uc de $\operatorname{BN}(N=1,2, \cdots, 6)$. A polarização é definida como: $P[\%]=\frac{I_{\uparrow}-I_{\downarrow}}{I_{\uparrow}+I_{\downarrow}} . \ldots 128$

8.17 Magnetoresistência de tunelamento (TMR) em função da voltagem fonte dreno, $V_{d s}$, para $N$ uc de $\mathrm{BN}(N=1,2, \cdots, 6) \ldots \ldots \ldots \ldots$

A.1 Grid unidimensional. . . . . . . . . . . . . . . . . 135

A.2 Grid bidimensional. . . . . . . . . . . . . . . . . . . 137

A.3 Ordenamento dos pontos do grid no método Red-Black Gauss-Seidel. (a) Grid unidimensional. (b) Grid bidimensional. . . . . . . . . . . . . . . . . . . . . . 144

A.4 Erros gerados a partir da aproximação $v_{i}=\sin \left(\frac{i k \pi}{N}\right)$ para os modos $k=$ $1,5,10$ para um grid com $N=64$ pontos. . . . . . . . . . . . . . 145

A.5 Norma dos erros $\left(\|e\|_{2}\right)$ para a aproximação $v_{i}=\operatorname{sen}\left(\frac{i k \pi}{N}\right)$ para os modos $k=$ 1, 5, 10 para um grid com $N=64$ pontos em função do número de iterações.

(a) Método de Jacobi e (b) Método de Gauss-Seidel . . . . . . . . . . . . . . 146

A.6 (a) Erro inicial dado pela equação A.49. (b) Erro após 5 iterações. (c) Erro após 50 iterações. . . . . . . . . . . . . . . . . . . . . . . 147

A.7 Pontos do fine grid $\Omega^{h}$ mapeados para o coarse grid $\Omega^{2 h}$. . . . . . . . . . . 149

A.8 Os valores em pontos do coarse grid, são mapeados para o fine grid sem nenhuma modificação. Os demais valores dos pontos no fine grid que não são pontos do coarse grid, são obtidos através de médias entre os vizinhos dos pontos no coarse grid. . . . . . . . . . . . . . . . . . . . .

A.9 Se o erro exato em $\Omega^{h}$ (Representado pela linha vermelha) é suave, o erro obtido da interpolação do coarse grid $e^{2 h}$ (linha azul) deve fornecer uma boa representação do erro exato no fine grid. 
A.10 Se o erro exato em $\Omega^{h}$ (indicado pela linha vermelha) for oscilatório, o resultado do erro obtido a partir da interpolação no coarse grid ( indicado por azul) não irá fornecer uma boa aproximação para o erro no fine grid. . . . . . . . . . . 152

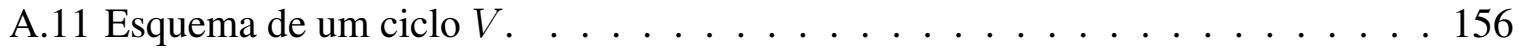

A.12 Esquema de um ciclo $F M G$. . . . . . . . . . . . . . . . . 157 


\section{Lista de Tabelas}

4.1 Energias de formação (eV) e distâncias de equilíbrio $(\AA ̊$ ) da bicamada de grafeno. 42

5.1 Energias de formação e distâncias de equilíbrio das FLG . . . . . . . . . . 58

6.1 Diferenças de energia total para os seis empilhamentos considerados. . . . . . . 89

8.1 Propriedades estruturais das nanofitas de grafeno dopadas com boro e nitrogênio 119 


\section{Capítulo 1}

\section{Introdução}

A combinação da Teoria, Modelagem e Simulação (TMS) desempenha um papel importante em todas as áreas da nanotecnologia. Essas três componentes distintas formam uma das ferramentas mais poderosas no desenvolvimento de novas tecnologias. Uma teoria pode ser definida como um conjunto de princípios científicos - como por exemplo a Teoria do Funcional da Densidade. A modelagem é a aplicação analítica/numérica de uma teoria para resolver problemas específicos. A simulação tem como objetivo tratar fielmente o problema físico com o maior número de detalhes possível, de modo que as características críticas e importantes do problema surjam de forma natural.

Um dos principais desafios na indústria computacional de hoje em dia está na miniaturização dos componentes de um circuito integrado. Muito esforço em pesquisa e desenvolvimento industrial é dedicado à diminuição dos transistores dos chips semicondutores. No entanto, esta tendência de miniaturização não pode durar para sempre dentro do atual paradigma do silício.

Um ponto importante quando atinge-se a escala nanométrica refere-se aos efeitos quânticos que estão relacionados com a estrutura atômica do sistema. Tais efeitos apresentam um sério desafio, pois exigem abordagens radicalmente diferentes das utilizadas na tecnologia do silício. $\mathrm{Na}$ escala onde a configuração espacial dos átomos torna-se importante, um átomo faz toda a diferença. Com o surgimento da nanoeletrônica e da spintrônica, o efeito da estrutura cristalina do material passa a ser utilizado em favor do dispositivo. É neste ponto que a TMS pode ser fundamental, auxiliando no entendimento das propriedades de novos materiais bem como no desenvolvimento de novas abordagens e métodos para serem utilizados na nanoeletrônica e na spintrônica.

No campo da nanoeletrônica, o principal desejo é a obtenção de um nanotransistor que 
substitua os atuais baseados no silício. Porém, para a utilização de um material como um transistor, é desejável que este possua um gap de energia. O gap eletrônico é uma propriedade intrínseca de materiais semicondutores isolados, determinando em grande parte as propriedades óticas e do seu transporte eletrônico. Como tal, o gap eletrônico tem um papel fundamental na física e tecnologia dos materiais semicondutores, pois é ele que regula o funcionamento desses sistemas. Um gap de energia ajustável através de um campo elétrico externo é altamente desejado, pois iria permitir uma grande flexibilidade no projeto e otimização dos dispositivos. No entanto, em matérias-primas convencionais, o gap é determinado pela estrutura cristalina do material, impedindo tal controle. É neste ponto que entra o grafeno, onde recentemente na literatura, foram construídos vários dispositivos usando bicamadas de grafeno. Foi demonstrado que através da aplicação de um campo elétrico externo por um eletrodo de gate, ocorre o surgimento de um gap de energia continuamente ajustável, dependendo somente da intensidade do campo presente na bicamada de grafeno [6,7]. Combinado às notáveis propriedades de transporte eletrônico com o controle do gap de energia, o grafeno torna-se um potencial candidato para a aplicação em novos dispositivos eletrônicos de alto desempenho.

A spintrônica [8] visa combinar os campos tradicionalmente separados do armazenamento de informação magnética e dos semicondutores eletrônicos, para a construção de componentes mais poderosos, que exploram o spin do elétron, além de somente a sua carga. O exemplo que melhor ilustra o potencial da spintrônica é a chamada Magnetic Random Access Memory (MRAM) [9], que combina em um único dispositivo as vantagens convencionais do armazenamento magnético de dados (discos rígidos) e a eletrônica convencional das memórias de acesso randômico (RAM). Estes dispositivos integram ao mesmo tempo, células de memória nãovoláteis com a velocidade das células RAM convencionais. Um ingrediente essencial para a spintrônica é a geração de correntes eletrônicas spin-polarizadas, que normalmente são realizadas pela passagem de correntes elétricas através de um metal ferromagnético.

As válvulas de spin são um outro sistema básico da spintrônica, ou seja, dispositivos que mudam de resistividade em função da polarização do spin da corrente. Um exemplo de válvula de spin real são os materiais que apresentam a chamada magneto-resistência gigante (GMR) [10]. Tais sistemas são constituídos por multicamadas alternadas de um metal magnético e não-magnético, exibindo uma forte sensibilidade da corrente elétrica com a orientação relativa das magnetizações das camadas magnéticas [10]. Logo após sua descoberta em 1988, o efeito 
da GMR foi explorado para melhorar a sensibilidade das cabeças de leitura de discos rígidos. Dessa maneira, a GMR permitiu a diminuição do tamanho dos bits magnéticos, aumentando a densidade de armazenamento de dados nos discos rígidos de forma dramática. A integração dos campos da nanoeletrônica com o da spintrônica é uma abordagem promissora para a realização de dispositivos em nanoescala. Dentre os diversos materiais que podem ser utilizados, o grafeno (tema central da tese) realiza essa integração de maneira natural.

Nesta tese, investigaremos a partir de cálculos de primeiros princípios, dispositivos e componentes de dispositivos baseados em grafeno. Abordaremos tanto os campos da nanoeletrônica como o da spintrônica. O ponto de partida para esta investigação, é a utilização do formalismo de funções de Green fora do equilíbrio (NEGF) juntamente com a teoria do funcional da densidade (DFT) para o estudo das propriedades de transporte eletrônico dos sistemas. O texto da tese é dividido da seguinte maneira. No capítulo 2 apresentaremos uma introdução sobre o grafeno, elucidando o estado da arte das tecnologias atuais que utilizam este material e quais os desafios existentes. No capítulo 3 apresentaremos a fundamentação teórica utilizada no desenvolvimento da tese, onde na seção 3.1, faremos uma breve introdução sobre a Teoria do Funcional da Densidade (DFT), que será a base teórica para todo o nosso estudo e desenvolvimento. Na seção 3.2, apresentaremos de maneira detalhada o formalismo de Funções de Green Fora do Equilíbrio, onde esta técnica acoplada com a DFT foi implementada no código TRANSAMPA para os cálculos de transporte eletrônico. Na seção 3.3 discutiremos os principais aspectos da técnica de multigrid que foi implementada no código SIESTA para a solução da equação de Poisson no espaço real, juntamente com alguns resultados de testes de validação da implementação. Uma descrição detalhada de toda a teoria envolvida neste desenvolvimento foi deixada para o apêndice A. No capítulo 4, iniciaremos com a apresentação dos resultados da tese, onde neste capítulo encontra-se os resultados sobre um nanotransistor de bicamada de grafeno. Seguindo com os resultados, no capítulo 5, apresentaremos os resultados sobre as multicamadas de grafeno, onde elucidamos as propriedades de transporte desse material e a sua dependência com o tipo de empilhamento das camadas de grafeno. No capítulo 6, apresentaremos os resultados para sistemas de grafeno sobre um substrato de nitreto de boro hexagonal (h-BN), onde investigamos tanto o h-BN isolado quanto ele servindo de suporte para bicamadas de grafeno. No capítulo 7, apresentaremos os resultados de um estudo das componentes em separado de um nanotransistor de grafeno, onde são utilizados como canal uma bicamada 
de grafeno, como dielétrico e substrato o h-BN e como contatos o cobre. Neste capítulo estudaremos principalmente as propriedades eletrônicas desse sistema na presença de campos elétricos externos. No capítulo 8, apresentaremos os resultados pertinentes aos dispositivos com aplicação na spintrônica, onde evidenciaremos as propriedades do transporte eletrônico de nanofitas de grafeno pristinas e dopadas com boro e nitrogênio. Dentro ainda deste capítulo, apresentaremos um estudo de uma junção túnel magnética, onde elucidaremos a sua utilização como filtros de spin e elementos para dispositivos de magnetoresistência gigante (GMR). Por fim no capítulo 9, apresentaremos um resumo das conclusões obtidas. 


\section{Capítulo 2}

\section{Grafeno}

'Diamante, grafite, carvão, combustíveis fósseis, dióxido de carbono, DNA, etc...'. Alótropo ou não, o carbono pode ser encontrado em muitas formas - cerca de 10 milhões de compostos. É o elemento mais abundante em nossas vidas. Seu comportamento complexo tem sido amplamente estudado ao longo dos anos. Acredita-se que o conhecimento sobre o diamante ${ }^{1}$ seja muito antigo, entretanto, o grafite não era conhecido antes do século 18. Nos tempos modernos, o carbono foi identificado como um componente chave dos compostos naturais, como a carga genética na forma de DNA e em processos como a fotossíntese. O carbono também participa de reações solares como as colisões de partículas alfa. Na ciência em nanoescala, o carbono e suas formas alotrópicas possuem uma área exclusiva para a sua pesquisa. Uma infinidade de estudos foram elaborados sobre as propriedades de alótropos de carbono em nanoescala. O mundo foi levado a uma nova percepção, com importantes repercussões na forma de aplicações, em particular transistores, com a descoberta dos fulerenos [11] e nanotubos de carbono [12]. Na figura 2.1 mostramos imagens das formas alotrópicas do carbono descritas anteriormente.

A história de grafeno pode ser considerada como velha e nova. Primeiramente foi postulado em 1947 por J.C. Wallace [13] como algo puramente teórico, construído para resolver o problema do cálculo da estrutura de bandas do grafite. Em 1984, G. Semenoff [14], utilizou o modelo proposto por Wallace, como um modelo em matéria condensada para o entendimento de uma anomalia em três dimensões e em 1988 D. Haldane [15] utilizou como modelo para um Efeito Hall Quântico (QHE) sem níveis de Landau. Na década de 90 foi utilizado como ponto de partida para o cálculo da estrutura de bandas de nanotubos de carbono [16]. Mas ninguém até o momento havia pensado que um dia seria possível fabricar este material livre.

\footnotetext{
${ }^{1}$ do grego Adamas - o indestrutível
} 


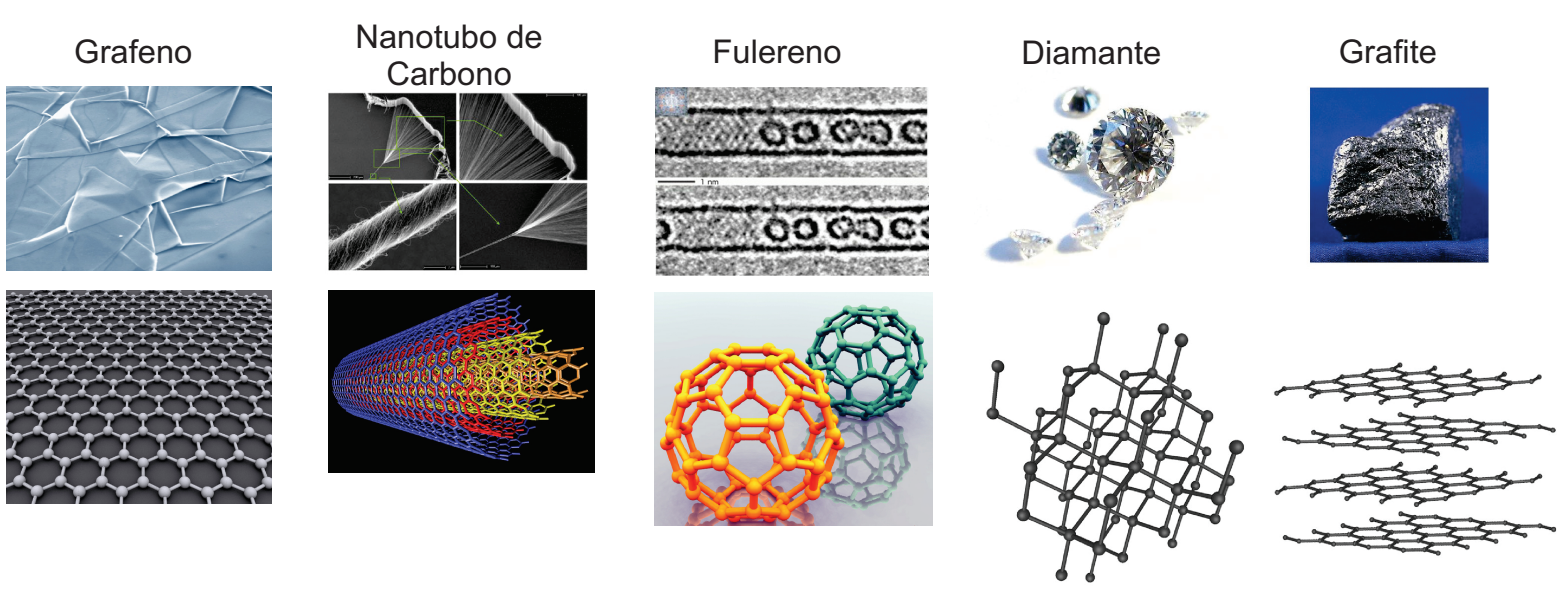

Figura 2.1: Formas alotrópicas do carbono.

Tal ceticismo provinha do influente teorema Mermin-Wagner [17], que durante a última parte do século passado foi interpretado com o significado que os cristais $2 \mathrm{D}$ não poderiam existir na natureza. Na verdade não se encontra naturalmente cristais 2D livres, e simulações computacionais mostram que eles não se formam espontaneamente, pois são termodinamicamente instáveis [18]. É neste cenário que a realização de grafenos livres veio como uma surpresa enorme. Mas em um exame mais atento não deve ter sido. O teorema Mermin-Wagner não exclui a existência de cristais de tamanho finito em 2D: sua validade é limitada a sistemas infinitos com interações de curto alcance no estado fundamental. Embora cristais 2D não se formem espontaneamente, eles podem existir e são perfeitamente estáveis quando empilhados e mantidos juntos por forças de Van der Waals, como parte de uma estrutura 3D como o grafite.

Em 2004, uma equipe da Universidade de Manchester liderada por Andre Geim demonstrou um processo de esfoliação mecânica simples [19], pela qual o grafeno, um cristal bidimensional (2D) de somente um átomo de espessura, formado somente de átomos de carbono, arranjados em uma rede hexagonal [5], pode ser isolado a partir do grafite. Na figura 2.2 mostramos imagens do grafeno isolado experimentalmente.

$\mathrm{O}$ isolamento do grafeno e as medidas subsequentes que revelaram as suas extraordinárias propriedades eletrônicas [20], desencadeou um frenesi de atividade científica com uma magnitude que nunca foi vista. Ele rapidamente atravessou fronteiras disciplinares e, em maio de 2010, o simpósio do Nobel em Estocolmo, foi sobre o grafeno. Neste evento histórico, o grafeno era a peça central de animadas interações entre os pesquisadores que raramente compartilham um terreno comum: físicos, químicos, biólogos, engenheiros e físicos em teoria de 


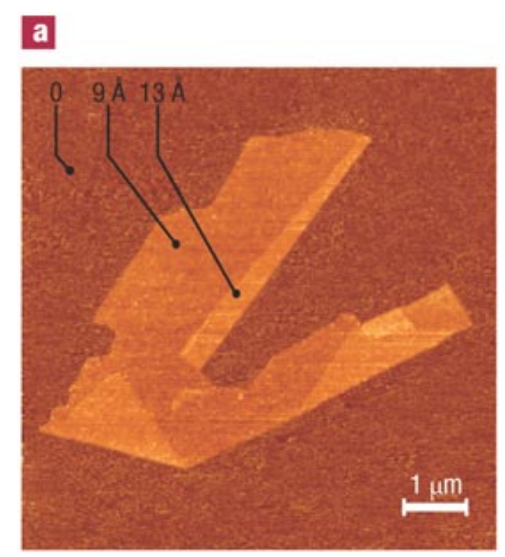

b

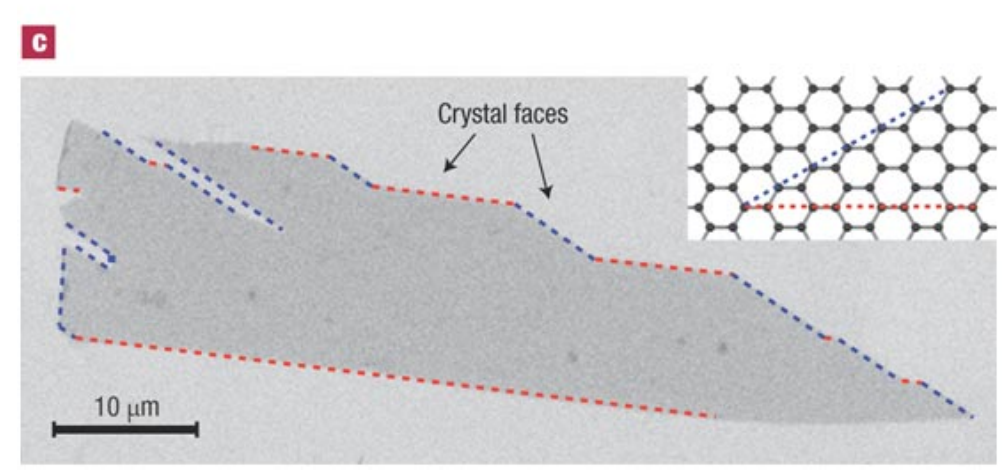

Figura 2.2: Grafeno, um cristal de uma única camada: o mais fino material já isolado na natureza. (a) Imagem de microscopia de força atômica de um grafeno. (b) Uma única folha de grafeno suspensa livremente entre contatos metálicos. (c) Imagem de microscopia eletrônica de varredura de um único cristal de grafeno. Esta imagem mostra que a maioria das bordas desse sistema possui uma orientação zig-zag e arm-chair, indicada pelas linhas em azul e vermelho, respectivamente. Imagem retirada da referência [1].

campo. O entusiasmo sobre o grafeno se estende para além das suas propriedades eletrônicas incomuns. Tudo sobre o grafeno - sua composição química, propriedades mecânicas, térmicas e ópticas - é diferente de maneiras interessantes.

Estruturalmente, o grafeno é definido como uma folha planar de somente um átomo de espessura, composta por átomos de carbono arranjados em uma rede cristalina hexagonal, como ilustrado na figura 2.3(a). Cada átomo de carbono no grafeno é ligado três primeiros vizinhos por uma ligação planar forte do tipo $\sigma$, que envolve três dos seus elétrons de valência ocupando os orbitais híbridos $s p^{2}$. No equilíbrio a ligação $\sigma$ carbono-carbono possui um comprimento de $0.142 n m$ e um ângulo de $120^{\circ}$. Essas ligações são responsáveis pela estrutura planar do grafeno e por suas propriedades mecânicas e térmicas. O quarto elétron de valência que sobra no orbital semi-preenchido $2 p_{z}$, ortogonal ao plano do grafeno, forma uma ligação fraca do tipo $\pi$, através do overlap com outros orbitais $2 p_{z}$. Os elétrons $\pi$ são delocalizados e determinam as 
propriedades de transporte do grafeno.
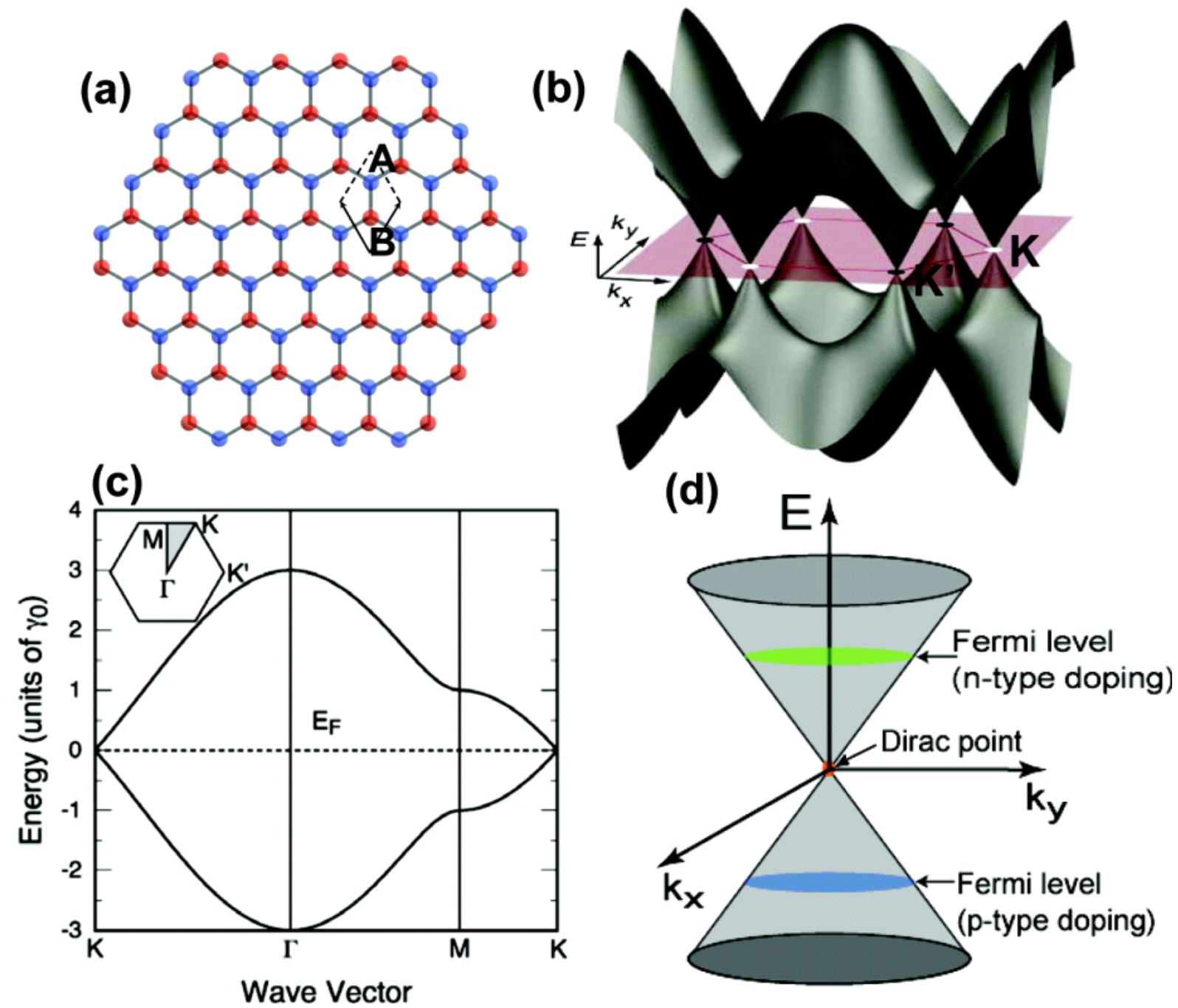

Figura 2.3: (a) Rede hexagonal do grafeno com dois átomos por célula unitária, A e B. (b) Estrutura de bandas $3 D$ do grafeno. (c) Estrutura de bandas do grafeno. (d) Aproximação de baixas energias para a estrutura de bandas do grafeno, onde dois cones tocam-se no ponto de Dirac. A posição do nível de Fermi em relação ao ponto de Dirac determina o tipo de dopagem no grafeno. Imagem retirada na referência [2].

Três ingredientes são utilizados para produzir as propriedades eletrônicas incomuns do grafeno: a sua estrutura 2D, a rede cristalina hexagonal e o fato de que todos os sítios na rede são ocupados pelos mesmos átomos, o que introduz uma simetria de inversão. Notamos que a rede hexagonal não é uma rede Bravais. Em vez disso, ela pode ser vista como uma rede bipartida, composta de duas subredes triangulares interpenetrantes, A e B, e com cada átomo na subrede A tendo apenas átomos vizinhos da subrede $B$ e vice-versa. No caso do grafeno, os átomos que ocupam as duas subredes são idênticos e, como veremos isto tem implicações importantes para 
a sua estrutura de bandas eletrônicas. Como mostrado na 2.3(a), os átomos de carbono na subrede A estão localizados nas posições $\vec{R}=m \vec{a}_{1}+n \vec{a}_{2}$, onde $m, n$ são inteiros e $\vec{a}_{1}=\frac{a}{2}(3, \sqrt{3})$, $\vec{a}_{2}=\frac{a}{2}(3,-\sqrt{3})$ são os vetores de translação para a subrede A. Os átomos na subrede $B$ estão localizados em $\vec{R}+\vec{\tau}$, onde $\vec{\tau}=\left(\vec{a}_{2}+\vec{a}_{1}\right) / 3$. Os vetores da rede recíproca, $\vec{G}_{1}=\frac{2 \pi}{3 a}(1, \sqrt{3})$, $\vec{G}_{2}=\frac{2 \pi}{3 a}(1,-\sqrt{3})$ e a primeira zona de Brillouin, um hexágono com os vértices nos chamados pontos $K$, são mostrados na figura 2.3b-d. Somente dois dos pontos $K$ são inequivalentes, e os outros são conectados pelos vetores da rede recíproca. As propriedades eletrônicas do grafeno são controladas pela relação de dispersão cônica de baixa energia nesses pontos $K$.

Os estados eletrônicos de baixa energia, que são determinados pelos elétrons que ocupam os orbitais $p_{z}$, podem ser derivadas a partir de uma Hamiltoniana tight-binding [13] no modelo de Huckel para interações de primeiros vizinhos:

$$
H=-t \sum_{|\vec{R}\rangle}\left(|\vec{R}\rangle\langle\vec{R}+\vec{\tau}|+| \vec{R}\rangle\left\langle\vec{R}-\vec{a}_{1}+\vec{\tau}|+| \vec{R}\right\rangle\left\langle\vec{R}-\vec{a}_{2}+\vec{\tau}\right|+\text { h.c. }\right)
$$

onde $\langle\vec{r} \mid \vec{R}\rangle=\Psi_{p_{z}}(\vec{R}-\vec{r})$ é a função de onda do orbital $p_{z}$ de um átomo em uma sub-rede A, $\langle\vec{r} \mid \vec{R}+\vec{\tau}\rangle$ é um estado similar de um átomo em uma sub-rede $\mathrm{B}$, e $t$ é a integral de hopping a partir de um estado em um átomo em A para um átomo adjacente em B. Os elementos de hopping na matriz, acoplam os estados nas subredes A com os estados na subrede B, e viceversa. É escolhido $t \approx 2.7 \mathrm{eV}$, tal que a estrutura de bandas tight-binding perto do ponto $K$ seja igual à obtida de um cálculo de primeiros princípios. Como existem duas sub-redes de Bravais, são necessários dois conjuntos de orbitais de Bloch, um para cada sub-rede, para a construção dos auto-estados de Bloch da Hamiltoniana:

$$
\begin{array}{r}
\left|\vec{k}_{A}\right\rangle=\frac{1}{\sqrt{N}} \sum_{\vec{R}} e^{i \vec{k} \cdot \vec{R}}|\vec{R}\rangle \\
\left|\vec{k}_{B}\right\rangle=\frac{1}{\sqrt{N}} \sum_{\vec{R}} e^{i \vec{k} \cdot \vec{R}}|\vec{R}+\vec{\tau}\rangle .
\end{array}
$$

Essas funções bloco-diagonalizam a hamiltoniana mono-eletrônica em $2 \times 2$ sub-blocos, com os elementos da diagonal nulos e os elementos fora da diagonal dados por:

$$
\left\langle\vec{k}_{A}|H| \vec{k}_{B}\right\rangle=-t e^{i \vec{k} \cdot \vec{\tau}}\left(1+e^{-i \vec{k} \cdot \vec{a}_{1}}+e^{-i \vec{k} \cdot \vec{a}_{2}}\right) \equiv e(\vec{k})
$$

As energias de Bloch de uma partícula $\epsilon(\vec{k})= \pm|e(\vec{k})|$ fornecem a estrutura de bandas na 
figura 2.3(b,c), com $\epsilon(\vec{k})=+|e(\vec{k})|$ correspondendo a banda de condução $\pi^{*}$ e $\epsilon(\vec{k})=-|e(\vec{k})|$ correspondendo a banda de valência $\pi$. No ponto de alta simetria $K \epsilon(\vec{k})=0$. Esses pontos são chamados de pontos de Dirac.

Um ponto importante na discussão da física do grafeno é o comportamento do espectro de energia e das autofunções para cada ponto $k$ perto do ponto de Dirac. Como somente dois pontos $K$ são inequivalentes, necessitamos de focar em somente um deles. Seguindo a convenção, chamados esses pontos de $K$ e $K^{\prime}$. Para o ponto $K$, é conveniente definir um vetor $\vec{q}=\vec{K}-\vec{k}$. Expandindo ao redor de $\vec{q}=0$, e substituindo $\vec{q} \rightarrow-i \hbar\left(\partial_{x}, \partial_{y}\right)$, a equação de auto-valores torna-se:

$$
H_{K} \Psi_{K}=-i \hbar v_{F}\left(\begin{array}{cc}
0 & \partial_{x}-i \partial_{y} \\
\partial_{x}+i \partial_{y} & 0
\end{array}\right)\left(\begin{array}{c}
\psi_{K A} \\
\psi_{K B}
\end{array}\right)=\epsilon\left(\begin{array}{c}
\psi_{K A} \\
\psi_{K B}
\end{array}\right)
$$

onde $v_{F}=\frac{\sqrt{3}}{2} \frac{a t}{\hbar} \approx 10^{6} \mathrm{~m} / \mathrm{s}$ é a velocidade de Fermi das quasipartículas. As duas componentes $\Psi_{K A}$ e $\Psi_{K B}$ fornecem a amplitude da função de onda nas subredes A e B. O operador acopla $\Psi_{K A}$ a $\Psi_{K B}$, mas não a ele mesmo, desde que o hopping de primeiros vizinhos em uma rede hexagonal, acopla somente os sítios A e B. Os autovalores são lineares em magnitudes de $q$ e não dependem da sua direção, $\epsilon(q)= \pm \hbar v_{F}|\vec{q}|$ produzindo uma banda de elétrons e buracos conicamente simétricas, como mostrado na figura 2.3(d). A simetria elétron buraco na relação de dispersão de baixa energia é ligeiramente modificada quando overlaps de segunda, terceira e ordem superiores são incluídos. Entretanto, a degenerescência no ponto de dirac permanece inalterada mesmo quando correções de ordem superior são incluídas. A relação de dispersão linear implica em uma velocidade de grupo independente da energia, $v_{\text {grupo }}=|\partial E / \hbar \partial q|=$ $|\partial E / \hbar \partial q|=v_{F}$, para excitações de baixa energia, $(|E| \ll t)$.

As autofunções descrevendo as excitações de baixa energia perto do ponto $K$ são:

$$
\begin{array}{r}
\Psi_{K}^{ \pm}\left(\theta_{q}\right)=\left(\begin{array}{c}
\psi_{K A} \\
\psi_{K B}
\end{array}\right)=\frac{1}{\sqrt{2}}\left(\begin{array}{c}
e^{i \theta_{q} / 2} \\
\pm e^{-i \theta_{q} / 2}
\end{array}\right), \\
\theta_{q} \equiv \tan ^{-1}\left(q_{x} / q_{y}\right)
\end{array}
$$

Essa representação em duas componentes, que formalmente representam spin, correspondem à projeção da função de onda em cada uma das subredes.

Uma outra forma concisa de escrever a hamiltoniana na equação 2.1 é:

$$
H_{K}=\hbar v_{F} \vec{\sigma} \cdot \vec{p}
$$


onde $\vec{p}=\hbar \vec{q}$ e as componentes do operador $\vec{\sigma}=\left(\sigma_{x}, \sigma_{y}\right)$ são as matrizes usuais de Pauli, que agora operam no grau de liberdade das subredes, no lugar do spin, e por isso chamado de pseudospin. Formalmente, essa é exatamente a equação de Dirac-Weyl em 2D, tal que as excitações de baixa energia são descritas não somente pela equação de Schrödinger, mas no lugar, por uma equação que normalmente é utilizada para a descrição de partículas ultrarelativísticas (ou sem massa), de spin $1 / 2$, com a velocidade da luz substituída pela velocidade de Fermi, $v_{F}$, que é 300 vezes menor que $c$. Consequentemente as quasipartículas no grafeno, no regime de baixas energias, são chamadas de "férmions de Dirac sem massa".

A equação de Dirac-Weyl na eletrodinâmica quântica (QED) surge da equação de Dirac, tomando a massa de repouso da partícula como sendo zero. Isso resulta em duas equações descrevendo partículas com helicidade ou quiralidade opostas. A natureza quiral da equação de Dirac-Weyl é uma consequência direta da Hamiltoniana ser proporcional ao operador de helicidade: $\hat{h}=\frac{1}{2} \vec{\sigma} \cdot \hat{p}$, onde $\hat{p}$ é um vetor unitário na direção do momento. Como $\hat{h}$ comuta com a Hamiltoniana, a projeção do spin é uma quantidade conservada que pode ser tanto positiva quanto negativa, correspondendo ao spin e momento sendo paralelos ou antiparalelos um com o outro.

Em física da matéria condensada, excitações de buracos normalmente são vistas como um equivalente aos pósitrons. Entretanto, elétrons e buracos são normalmente descritos por equações de Schrödinger separadas, que não são de maneira alguma conectadas. Em contraste a isso, elétrons e buracos no grafeno são interconectados, exibindo propriedades análogas à simetria de conjugação de carga na QED. Isso é uma consequência da simetria cristalina, que exige funções de onda de duas componentes para definir as contribuições relativas às quasipartículas nas subredes A e B. A descrição em duas componentes do grafeno é muito similar à funções de onda spinoriais na QED, mas o índice de spin para o grafeno indica a subrede do sistema, no lugar do spin real dos elétrons. Isso permite introduzir a quiralidade no problema como sendo a projeção do pseudospin na direção do momento - que no ponto K, é positivo para elétrons e negativo para buracos. Dessa maneira, cada excitação de quasipartículas no grafeno possui a sua antipartícula. O par partícula-antipartícula corresponde ao par elétron-buraco com mesmo momento mas sinais opostos em energia e com quiralidade oposta. No ponto $K^{\prime}$ a quiralidade dos elétrons e buracos é invertida.

Uma consequência importante dos resultados anteriores é a supressão do retroespalhamento. 
A probabilidade de retroespalhamento pode ser obtida a partir da projeção da função de onda correspondendo a uma partícula se movimento positivamente, $\Psi_{K}^{+}(\vec{q}(\theta))$, na função de onda de uma partícula retroespalhada, $\Psi_{K}^{+}(\vec{q}(\theta+\pi))$. Com o mesmo ponto K nós temos: $\Psi_{K}^{+}(\vec{q}(\theta)) \rightarrow$ $\Psi_{K}^{+}(\vec{q}(\theta+\pi))=i \Psi_{K}^{-}(\vec{q}(\theta))$ que fornece $\left\langle\Psi_{K}^{+}(\vec{q}(\theta)) \mid \Psi_{K}^{-}(\vec{q}(\theta))\right\rangle=0$. Em outras palavras, o retroespalhamento é suprimido. Esta regra de seleção surge do fato que o retroespalhamento com o mesmo ponto $K$ inverte a direção do pseudospin. Podemos considerar também o retroespalhamento entre dois pontos $K$ distintos. Expandindo em $\overrightarrow{q^{\prime}}=\overrightarrow{K^{\prime}}-\vec{k}$ ao redor do segundo ponto de Dirac, $H_{K^{\prime}}=-\hbar v_{F} \overrightarrow{\sigma *} \cdot \vec{p}$, que está relacionado com $H_{K}$ por reversão temporal. A solução em $K^{\prime}$ é obtida tomando $p_{x} \rightarrow-p_{x}$ na equação 2.1 , o que resulta em

$$
\Psi_{K^{\prime}}^{ \pm}=\frac{1}{\sqrt{2}}\left(\begin{array}{c}
e^{-i \theta_{q} / 2} \\
\pm e^{-i \theta_{q} / 2}
\end{array}\right)
$$

Assim, o retroespalhamento entre os dois pontos $K$ diferentes também não é permitido pois ele ainda mantém a transformação $\Psi_{K}^{+}\left(\theta_{q}\right) \rightarrow \Psi_{K^{\prime}}^{+}\left(\theta_{q}+\pi\right)=i \Psi_{K}^{-}\left(\theta_{q}\right)$, que coloca a partícula em um estado que é ortogonal ao original. Essa regra de seleção vem do fato que o retroespalhamento entre dois $K$ distintos inverte a quiralidade da quasipartícula. Essas regras de seleção provenientes do retroespalhamento no grafeno, possuem importantes consequências experimentais, incluindo o transporte balístico em baixas temperaturas e condutividade extremamente alta à temperatura ambiente. Os portadores de carga do grafeno, no regime de transporte balístico, movem-se com uma velocidade de Fermi $v_{F} \approx 10^{6} \mathrm{~ms}^{-1}[21,22]$.

Um fator importante que afeta a mobilidade no grafeno é a densidade de portadores. Aumentando esta densidade na monocamada de grafeno, geralmente a mobilidade decresce, com exatamente o mesmo fator, dependendo da natureza dos centros espalhadores dominantes [23, 24]. Em grafenos suspensos e exfoliados, onde as interações com os substratos são eliminados e a presença de defeitos provenientes do crescimento do material são reduzidas, foram observadas mobilidades ao redor de $200000 \mathrm{~cm}^{2} V^{-1} s^{-1}$ [21,22]. Quando o grafeno está sobre um substrato isolante, como o óxido de silício amorfo, $a-\mathrm{SiO}_{2}$, dependendo da natureza e pureza do isolante, a mobilidade é significantemente menor: apenas algumas milhares a dezenas de milhares $\mathrm{cm}^{2} V^{-1} \mathrm{~s}^{-1}$. Em substratos monocristalinos, como nitreto de boro hexagonal (h-BN), foram encontradas mobilidades muito altas [25]. Atualmente, grafenos crescidos epitaxialmente e por CVD (Chemical Vapor Deposition) mostram mobilidades mais baixas, na ordem de apenas alguns milhares de $\mathrm{cm}^{2} V^{-1} \mathrm{~s}^{-1}[26]$. 
Semicondutores convencionais com gaps de energia $>0.5 \mathrm{eV}$ e nanotubos semicondutores que possuem gaps que escalam inversamente com o diâmetro, propiciam a construção de ótimos dispositivos que são ligados e desligados ${ }^{2}$ através da aplicação de potenciais de gate, onde a razão on/off da corrente chega a valores da ordem de $10^{4}-10^{7}$. Esses valores são apropriados para dispositivos lógicos. O grafeno, por outro lado, não possui um gap de energia e consequentemente não possui essa capacidade. O ligar/desligar do grafeno é controlado unicamente pela variação da densidade de estados (DOS) com a energia de Fermi, $E_{F}$. Experimentalmente, a maioria dos estudos dos transistores de efeito de campo (FET) de grafeno (GFET), obtém razões $I_{o n} / I_{o f f} \leq 10$. Na figura 2.4 mostramos um exemplo da mudança na condutância de um transistor de efeito de campo de grafeno em função do potencial de top-gate/back-gate. Estes potenciais controlam a intensidade do campo elétrico no canal do transistor bem como a posição do ponto de Dirac.

(a)

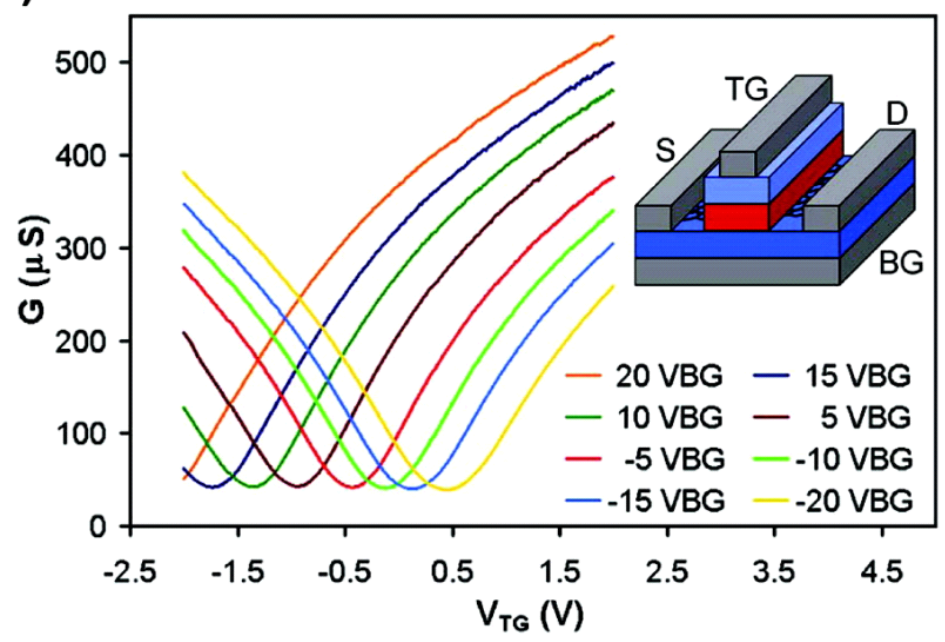

(b)

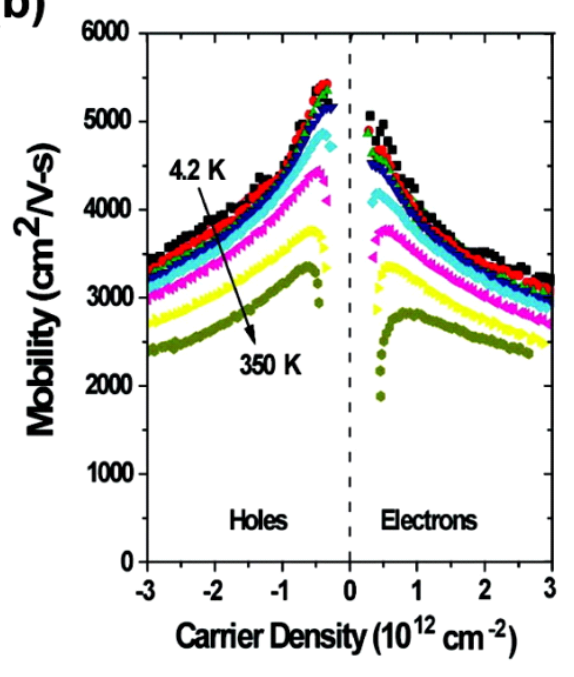

Figura 2.4: (a) Condutância de uma única camada de grafeno utilizada como canal em um transistor de efeito de campo, em função da voltagem de top gate, para diferentes voltagens de back gate. O inset mostra esquematicamente o modelo do dispositivo utilizado. Imagem retirada da referência [3]. (b) Mobilidades para elétrons e buracos de uma única camada de grafeno exfoliada e incorporada como canal de um transistor com um substrato de $\mathrm{SiO}_{2}$. Imagem retirada da referência [4]

Então, se o grafeno não é apropriado para dispositivos lógicos, porque existe esse grande interesse na eletrônica dos dispositivos de grafeno? O ponto fundamental do grafeno está na fantástica mobilidade dos portadores de carga, na ótima transcondutância dos dispositivos de grafeno e sua característica planar (2D). Essas características, sugerem que o grafeno pode ser

\footnotetext{
${ }^{2} \mathrm{O}$ estado ligado/desligado de um transistor corresponde à configuração de corrente máxima/mínima
} 
um material ideal para dispositivos de rádio frequiência. Nesses tipos de dispositivos, não é necessário que o sistema seja desligado, ou seja, apresente um gap de energia [27]. Porém, quando deseja-se utilizar

Para abrir um gap de energia no grafeno, e utilizar este material como um nanotransistor, é necessário a presença de um potencial que quebre a simetria entre os dois átomos na célula unitária. Entretanto, estes átomos estão separados por somente $0.14 \mathrm{~nm}$, e conseqüentemente, técnicas como litografia são impossíveis de serem utilizadas. Uma outra abordagem que vem sendo muito utilizada é a restrição da dimensionalidade do grafeno de $2 D$ para $1 D$, cortando o grafeno em fitas estreitas, as nanofitas de grafeno (GNR). Se as GNR's forem estreitas o suficiente, estados $1 D$ confinados são formados como os de uma partícula em uma caixa estreita e longa. Dessa maneira ocorre a abertura de um gap de energia, que é inversamente proporcional à largura das GNR's. GNR's podem ser feitas a partir de técnicas de litografia [28-31], 'unzipping' de nanotubos de carbono [32], e mesmo a partir de síntese direta [33]. Para a obtenção de um gap de energia razoável, $\geq 0.5 \mathrm{eV}$, é necessário a utilização de nanofitas com uma largura constante entre $2-3 \mathrm{~nm}$. Infelizmente, as técnicas convencionais de litografia não possuem tal precisão. Além disso, problemas com a reprodutibilidade da largura da fita e rugosidade das bordas, tornam a produção em grande escala difícil, limitando o seu uso.

A monocamada de grafeno tem atraído a maior parte da atenção dos pesquisadores, todavia, recentemente tem crescido o interesse nos sistemas consistindo de algumas camadas de grafeno. A estrutura eletrônica e as propriedades das multicamadas de grafeno dependem fortemente do número de camadas empilhadas e do seu tipo de empilhamento. O empilhamento mais estável no grafite é o chamado $A B$, comumente conhecido como Bernal, onde metade dos átomos estão uns sobre os outros, e a outra metade sobre os centros dos hexágonos da rede. Em uma bicamada de grafeno $\mathrm{AB}$, conforme podemos observar na figura 2.5, mesmo com uma interação fraca entre as camadas de grafeno, ocorre a formação de uma estrutura de bandas que é única.

Dois conjuntos de bandas de valência e condução formam uma banda com um formato hiperbólico, conforme podemos observar na figura 2.6(a)(linhas tracejadas). Entretanto, o gap nesse sistema ainda é zero $[34,35]$. Uma abordagem para a abertura deste gap de energia foi proposta inicialmente em 2006 [36]. Ela envolve a aplicação de um campo elétrico externo, perpendicular ao plano das bicamadas. Com a aplicação do campo, ocorre uma quebra de simetria entre as duas camadas gerando a abertura do gap de energia. 

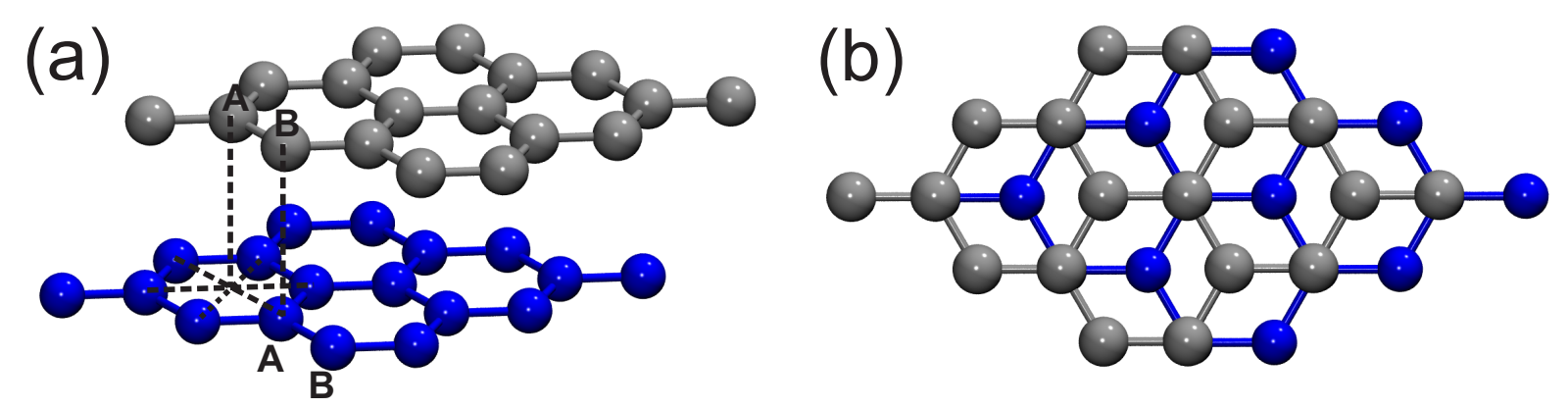

Figura 2.5: Visão esquemática da bicamada de grafeno com empilhamento de Bernal AB.

(a)

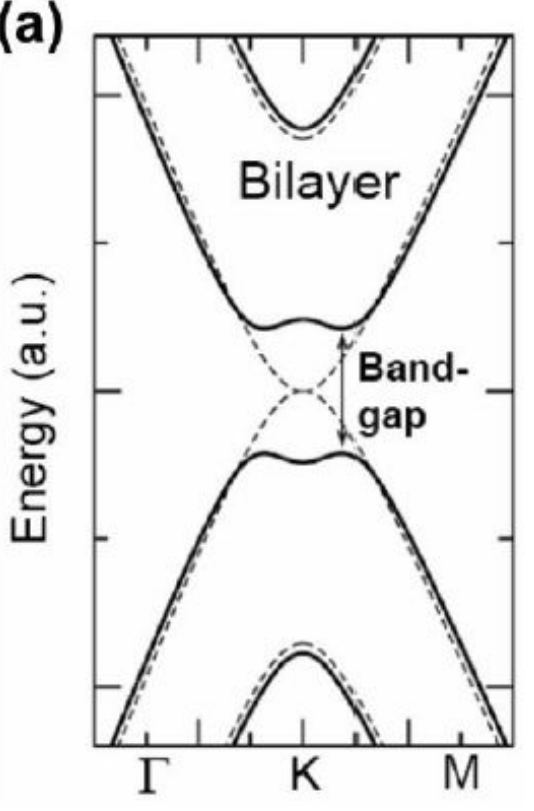

(b)

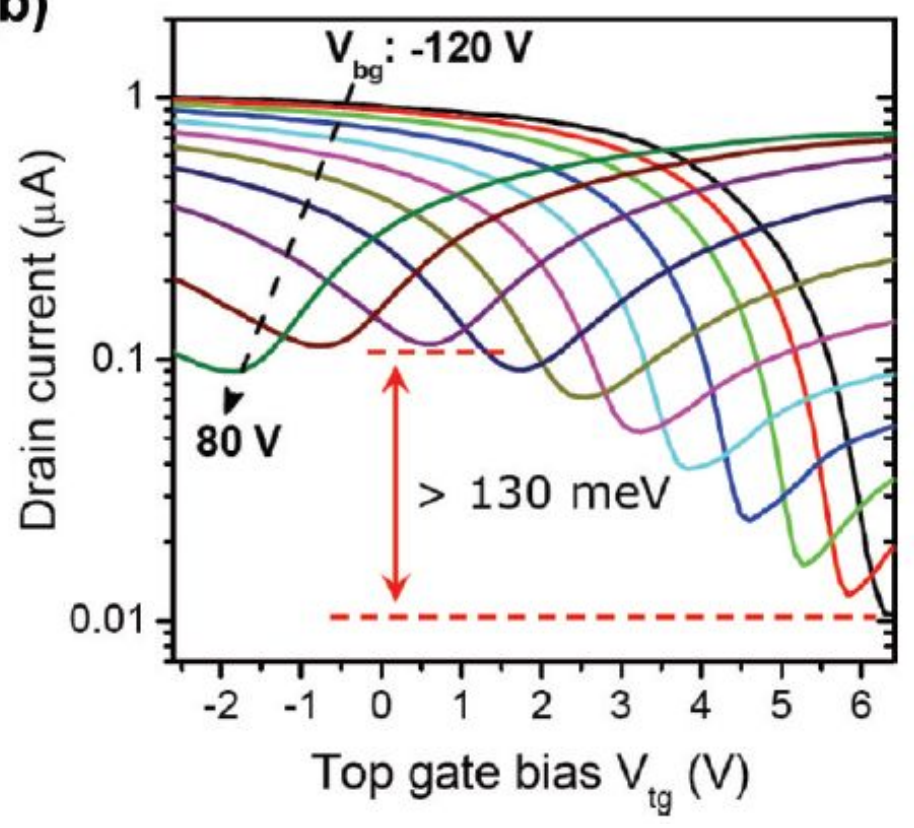

Figura 2.6: (a) Bandas de energia para uma bicamada de grafeno. Linha tracejada/cheia corresponde ao sistema na ausência/presença de campo elétrico aplicado. Imagem retirada da referência [5]. (b) Comportamento da corrente fonte-dreno para um transistor de bicamada de grafeno, em função da voltagem de top-gate. Imagem retirada da referência [2].

Na figura 2.6(a) mostramos esquematicamente como ocorre a abertura deste gap. Neste tipo de sistema, foi obtido razões on/off de aproximadamente 100 para a corrente à temperatura ambiente, enquanto que para a monocamada de grafeno, esta razão não passa de $5 . \mathrm{O}$ comportamento da corrente fonte-dreno em função da voltagem de top gate para um back gate fixo é mostrada na figura 2.6(b).

A partir desta breve introdução, observamos que existe um longo caminho de pesquisa para a utilização do grafeno como componente efetivo de algum dispositivo eletrônico. Do ponto de vista experimental, um requisito para aplicações de ponta, particularmente, em eletrônica e 
fotônica, é o controle completo sobre as propriedades físicas do material, ou seja, o tamanho lateral, homogeneidade na espessura da camada e pureza. O desenvolvimento de técnicas precisas para estabilizar e controlar o tipo de dopagem no grafeno também é muito importante. A preservação das propriedades intrínsecas do grafeno quando incorporado em dielétricos também é crítica, como também é a otimização das propriedades de contato entre o substrato e o grafeno. As multicamadas de grafeno, particularmente as bicamadas, também têm propriedades que podem ser usadas para novas aplicações e tecnologias. Entretanto, os métodos de síntese que produzem as bicamadas e multicamadas ainda são pouco precisos. 


\section{Capítulo 3}

\section{Fundamentação Teórica}

Neste capítulo iremos revisar alguns conceitos básicos fundamentais para o desenvolvimento da tese.

\subsection{Teoria do Funcional da Densidade - DFT}

A teoria do funcional da densidade, desenvolvida por Hohenberg e Kohn em 1964 [37] é um dos métodos de primeiros princípios de maior sucesso na descrição de um sistema de muitos elétrons interagentes. Nesta seção, descreveremos brevemente os aspectos básicos que levam a uma ampla aplicabilidade dessa teoria na física da matéria condensada. Uma revisão mais extensa desse tópico pode ser encontrada em [38, 39].

\subsubsection{Formulação do Problema}

Os elétrons em um sólido, molécula ou átomo, são descritos por uma função de onda de muitos corpos $\Psi\left(\left\{\vec{r}_{i}\right\}\right)$ das coordenadas $\vec{r}_{i}$ dos elétrons, que satisfaz a equação de Schrödinger de muitos corpos independente do tempo:

$$
\hat{H}|\Psi\rangle=E|\Psi\rangle,
$$

onde $\hat{H}$ é a hamiltoniana do sistema eletrônico interagente, contendo a energia cinética, $\hat{T}$, a interação coulombiana dos núcleos atômicos (caroços iônicos) $\hat{V}_{e-i}$ com os elétrons, e as interações elétron-elétron $\hat{V}_{e-e}$, que pode ser escrita como:

$$
\hat{H}=\hat{T}+\hat{V}_{e-i}+\hat{V}_{e-e}
$$


A equação de Schrödinger de muitos corpos descrita em (3.1), pode em princípio ser resolvida expandindo-se as funções de onda em uma base de determinantes de Slater, convertendo dessa maneira o problema em uma série de diagonalizações de matrizes. Na prática, isso pode somente ser feito em sistemas pequenos, consistindo de somente algumas dezenas de átomos. Uma das abordagens padrão para a solução da equação (3.1), é aproximação de Hartree-Fock (HF), que é a base para cálculos mais refinados na química quântica, como teorias de perturbação, ou interações de configurações (CI) [39].

Uma teoria alternativa ao formalismo de HF e a outros formalismos utilizados, é a teoria do funcional da densidade (DFT) onde a variável básica é a densidade eletrônica, no lugar da função de onda eletrônica de muitos corpos $\Psi\left(\left\{\vec{r}_{i}\right\}\right)$ :

$$
\rho(\vec{r})=N \int\left|\Psi\left(\vec{r}, \vec{r}_{2}, \cdots, \vec{r}_{N}\right)\right|^{2} d \vec{r}_{2} \cdots d \vec{r}_{N}
$$

que é uma quantidade muito mais simples para se trabalhar.

\subsubsection{Teoremas de Hohenberg-Kohn}

Os pilares da DFT são os dois teoremas de Hohenberg-Kohn (HK) [37], que reduz o problema interagente de $N$-elétrons a um problema de determinar a densidade eletrônica do estado fundamental $\rho(\vec{r})$. Os teoremas podem ser enunciados da seguinte forma:

Teorema 1 o potencial externo $v(\vec{r})$ sentido pelos elétrons é um funcional único da densidade eletrônica $\rho(\vec{r})$.

Teorema 2 A energia do estado fundamental $E[\rho]$, é mínima para a densidade $\rho(\vec{r})$ exata.

Conhecendo o funcional energia da densidade eletrônica, $E[\rho]$, pode-se então determinar a densidade eletrônica do estado fundamental, simplesmente através da minimização desse funcional da densidade, que pode ser muito mais simples que resolver a equação de Schrödinger de muitos corpos. Algo importante a notar, é que a DFT é em princípio uma teoria exata, que permite calcular a densidade exata do estado fundamental de um sistema de elétrons interagentes. 


\subsubsection{Equações de Kohn-Sham}

Os teoremas de Hohenberg-Kohn não fornecem uma receita prática para o cálculo do funcional energia da densidade eletrônica. Eles apenas declaram que este funcional existe. Uma maneira prática para o cálculo do funcional energia é dada pelas equações de KohnSham(KS) [40], que mapeia o sistema de elétrons interagentes com alguma densidade $\rho(\vec{r})$ em um sistema auxiliar não-interagente com a mesma densidade eletrônica do sistema interagente. Portanto, as equações de KS, são o ponto de partida para qualquer aplicação prática do DFT.

O funcional energia, $E[\rho]$, pode ser decomposto nas seguintes contribuições:

$$
E[\rho]=T[\rho]+E_{H}[\rho]+E_{x c}[\rho]+E_{e x t}[\rho]
$$

onde $T[\rho]$ é a energia cinética para a densidade, $\rho$, e é dada por:

$$
T[\rho]=\langle\Psi|\hat{T}| \Psi\rangle
$$

onde $|\Psi\rangle$ é a função de onda de muitos elétrons correspondente à densidade eletrônica $\rho$ para esse sistema. Note aqui, que não existe nenhuma forma explícita para o cálculo da energia cinética a partir da densidade $\rho$. Essa é uma das razões para não existir uma fórmula explícita para o funcional energia. $E_{H}[\rho]$ é o termo de Hartree que descreve a interação de repulsão coulombiana clássica da nuvem eletrônica. Para esse termo, existe uma fórmula explícita, que pode ser escrita para a sua dependência funcional na densidade eletrônica:

$$
E_{H}[\rho]=\frac{1}{2} \int \frac{\rho(\vec{r}) \rho\left(\vec{r}^{\prime}\right)}{\left|\vec{r}-\vec{r}^{\prime}\right|} d \vec{r} d \vec{r}^{\prime}
$$

$E_{x c}[\rho]$ é o que chamamos de termo de troca e correlação (XC), e novamente, também não existe uma fórmula explícita exata para ele. É, portanto, o outro termo além da energia cinética responsável pela impossibilidade de encontrar uma expressão explícita e exata para o funcional energia total. $\mathrm{O}$ termo de troca e correlação contém os efeitos quânticos puros como a interação de troca, devido ao princípio de exclusão de Pauli e os efeitos de correlação eletrônica. Esta contribuição é normalmente expressa em termos de uma integral sobre a densidade de elétrons e uma densidade de energia desconhecida XC, $\epsilon_{x c}[\rho](\vec{r})$ :

$$
E_{x c}[\rho]=\int d \vec{r} \rho(\vec{r}) \epsilon_{x c}[\rho(\vec{r})]
$$


Finalmente, o último termo $E_{\text {ext }}[\rho]$, representa a interação da nuvem eletrônica com o potencial externo devido aos núcleos atômicos. Esse termo pode ser escrito novamente de maneira explícita como um funcional da densidade eletrônica:

$$
E_{\text {ext }}[\rho]=\int d \vec{r} \rho(\vec{r}) V_{e-i}(\vec{r})
$$

A idéia básica do método de KS, é introduzir um conjunto auxiliar de funções de onda de um elétron, $\left\{\phi_{i}(\vec{r})\right\}$, onde estas geram a mesma densidade eletrônica $\rho(\vec{r})$ que a função de onda de muitos corpos $\Psi\left(\vec{r}_{1}, \vec{r}_{2}, \cdots, \vec{r}_{N}\right)$ :

$$
\rho(\vec{r})=\sum_{i=1}^{N} \phi_{i}^{*}(\vec{r}) \phi_{i}(\vec{r}),
$$

onde

$$
\left\langle\phi_{i} \mid \phi_{j}\right\rangle=\delta_{i j}
$$

Dessa maneira, podemos definir o funcional energia de Kohn-Sham, $T_{K S}[\rho]$, onde ele possa ser facilmente calculado a partir das funções de onda auxiliares de Kohn-Sham, $\phi_{i}$, como:

$$
T_{K S}[\rho]=\sum_{i=1}^{N}\left\langle\phi_{i}|\hat{T}| \phi_{i}\right\rangle .
$$

Entretanto, o termo de energia cinética de KS não é idêntico à energia cinética do sistema de elétrons interagentes,$T[\rho]$. A diferença entre a energia cinética de KS e a verdadeira energia cinética novamente é desconhecida, sendo esta absorvida dentro do funcional desconhecido de $\mathrm{XC}$ que é redefinido no método de KS como:

$$
E_{X C}[\rho] \rightarrow E_{X C}^{\prime}[\rho]=E_{X C}[\rho]+T[\rho]-T_{K S}[\rho]
$$

Assim, todos os efeitos de muitos corpos foram, agora, transferidos para o funcional de $\mathrm{XC}$, que é a única contribuição do funcional energia total que tem que ser aproximado. Todos os outros termos podem ser calculados exatamente com o método de KS. A principal vantagem do método de KS é que temos um conjunto eficaz de equações de Schrödinger de uma partícula (equações de Kohn-Sham) para as funções de onda auxiliares de KS, $\phi_{i}$, que por sua vez, fornecem a densidade eletrônica do estado fundamental do verdadeiro sistema de elétrons interagentes. Usando o princípio variacional, a energia total do estado fundamental do sistema 
deve ser estacionária com respeito a variação das funções de onda de KS:

$$
\frac{\delta E[\rho]}{\delta \phi_{i}}-\epsilon_{i} \phi_{i}=0
$$

onde $\epsilon_{i}$ são os multiplicadores de Lagrange que asseguram a ortogonalidade das funções de onda de Kohn-Sham, $\phi_{i}$, e irão fornecer todas as auto-energias efetivas de KS. As equações de KS são diretamente dadas por:

$$
\left(-\frac{\hbar^{2}}{2 m} \nabla^{2}+V_{H}[\rho](\vec{r})+V_{e x t}(\vec{r})+V_{X C}[\rho](\vec{r})\right) \phi_{i}(\vec{r})=\epsilon_{i} \phi_{i}(\vec{r}),
$$

onde

$$
V_{H}[\rho(\vec{r})]:=\int d \vec{r} \frac{\rho(\vec{r})}{|\vec{r}-\vec{r}|}
$$

é o potencial de Hartree devido à interação direta elétron-elétron e

$$
V_{X C}[\rho(\vec{r})]:=\frac{\delta E_{X C}[\rho]}{\delta \rho(\vec{r})}
$$

é o potencial efetivo de troca e correlação.

Deste modo, o problema interagente foi reduzido a um conjunto de equações de Schrödinger não-interagentes. Deve-se ressaltar que até agora não foram feitas aproximações. As equações de KS são exatas se conhecermos a forma exata do funcional de XC. A Hamiltoniana de KS:

$$
\hat{H}_{K S}=-\frac{\hbar^{2}}{2 m} \nabla^{2}+\hat{V}_{e x t}+\hat{V}_{H}[\rho]+\hat{V}_{X C}[\rho]
$$

depende da densidade eletrônica $\rho(\vec{r})$, que é a quantidade a ser determinada pelas funções de onda de KS. Assim, as equações de KS formam um conjunto não linear de problemas de autovalores, que deve ser resolvido de maneira auto-consistente.

\subsection{Formalismo de Funções de Green: Transporte Eletrônico}

O método de funções de Green fora do equilíbrio (NEGF) [41-48] é amplamente utilizado para a investigação das propriedades de transporte eletrônico de materiais em nanoescala, tais como uma única molécula entre eletrodos metálicos [49,50], fios atômicos [51,52], materiais baseados no carbono $[53,54]$, etc.

Na figura 3.1 mostramos esquematicamente as principais componentes do sistema utilizado 
em cálculos de transporte. As potenciais vantagens deste método são resumidas pelas seguintes características:

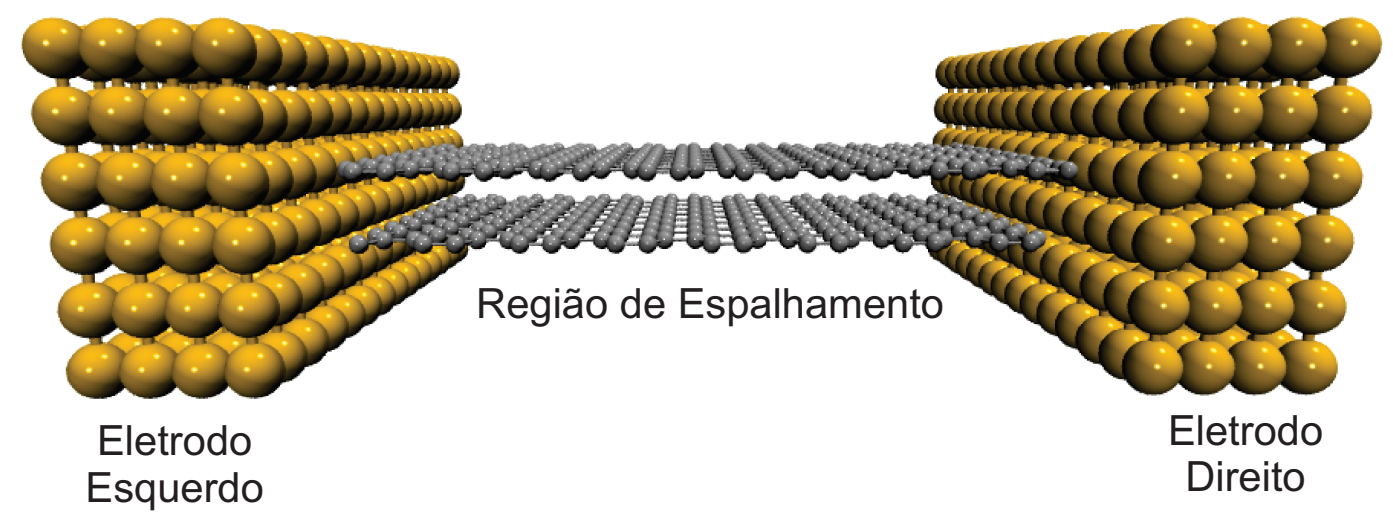

Figura 3.1: Esquema das componentes do sistema utilizado nos cálculos de transporte.

(i) Os contatos dos eletrodos esquerdo e direito ${ }^{1}$ são tratados utilizando a mesma abordagem teórica que para a região de espalhamento [41-48];

(ii) A estrutura eletrônica da região de espalhamento na presença de um bias finito entre os eletrodos, é resolvida de maneira auto-consistente, combinando-se a teoria de primeiros princípios do funcional da densidade (DFT) com o formalismo de funções de Green fora do equilíbrio (NEGF);

A maior parte dos aspectos práticos na implementação do método de NEGF combinado com o DFT, utilizando-se um conjunto de orbitais base localizados, PAO's, está no cálculo do estado fundamental do sistema em equilíbrio. Por isso, iniciaremos a nossa discussão a partir do cálculo da estrutura eletrônica do estado fundamental de um sistema no equilíbrio utilizando o método de funções de Green com o DFT.

\subsubsection{Funções de Green no equilíbrio}

Considerando um sistema hipotético, tridimensional, representado pela figura 3.2. O transporte eletrônico, se dá ao longo da direção $a / z$ e é permitido periodicidade nos planos $x y / b c$. A célula unitária da região central é denotada por $C_{0}$ e as células dos eletrodos esquerdo e direito são dadas por $L_{i}$ e $R_{i} \operatorname{com} i=0,1,2, \cdots$, respectivamente. Todas as células $L_{i}$ e $R_{i}$

\footnotetext{
${ }^{1}$ As vezes chamados de fonte e dreno.
} 


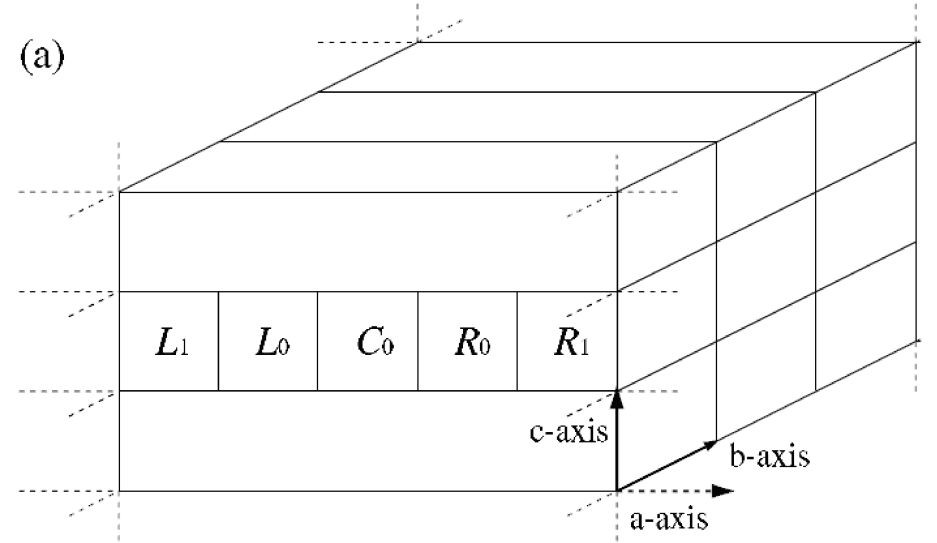

(b)

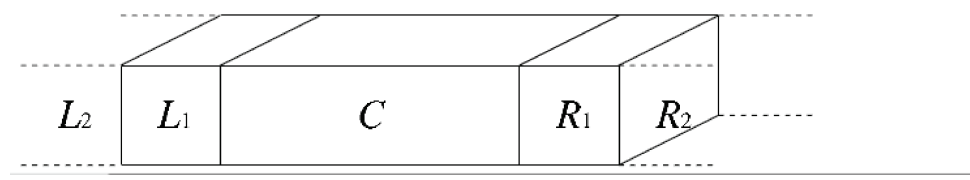

Figura 3.2: (a) Configuração do sistema tratado pelo método de funções de Green fora do equilíbrio (NEGF), contendo os eletrodos semi-infinitos esquerdo (L) e direito (R) ao longo do eixo $a$. Esses eletrodos podem possuir condições periódicas de contorno no plano $b c$. (b) Sistema unidimensional compactado a partir da configuração (a) utilizando a condição de periodicidade no plano $b c$, onde a região $C$ é a região central estendida consistindo de $C_{0}, L_{0}$ e $R_{0}$.

são arranjadas de maneira semi-infinita, cada uma contendo o mesmo número de átomos e a mesma configuração espacial. As células $L_{i}$ e $R_{i}$ podem ser diferentes uma da outra.

No estado de equilíbrio, quando o sistema possui um potencial químico em comum, a estrutura eletrônica pode ser determinada através de um cálculo DFT. Devido a periodicidade no plano $x y / b c$, a função de onda de Kohn-Sham (KS) do sistema pode ser expressa em termos de funções de Bloch usando orbitais $\phi_{i \alpha}$, localizados nos sítios $\tau_{i}$, como:

$$
\psi_{\sigma \nu}^{(\vec{k})}=\frac{1}{\sqrt{N}} \sum_{n} e^{i \vec{k} \cdot \vec{R}_{n}} \sum_{i \alpha} c_{\sigma \nu, i \alpha}^{(\vec{k})} \phi_{i}\left(\vec{r}-\tau_{i}-\vec{R}_{n}\right),
$$

onde $\sigma, \nu, i$ e $\alpha$ são os índices de spin, auto-estado, sítio e orbital base, respectivamente. $\mathrm{O}$ vetor de rede $\vec{R}_{n}$ e o vetor de onda de Bloch $\vec{k}$ são dados por:

$$
\begin{aligned}
& \vec{R}_{n}=l_{x} \hat{x}+l_{y} \hat{y}, \\
& \vec{k}=k_{x} \hat{\tilde{x}}+k_{y} \hat{\tilde{y}},
\end{aligned}
$$

onde $\hat{x}$ e $\hat{y}$ são os vetores da rede direta e $\hat{\tilde{x}}$ e $\hat{\tilde{y}}$ são os vetores da rede recíproca.

Considerando a variação da energia total do sistema expresso pela função de onda (3.18), 
com o DFT convencional, obtemos a seguinte equação de Kohn-Sham na forma matricial:

$$
H_{\sigma}^{(\vec{k})} c_{\sigma \nu}^{(\vec{k})}=\varepsilon_{\sigma \nu}^{(\vec{k})} S^{\vec{k}} c_{\sigma \nu}^{(\vec{k})}
$$

onde $c_{\sigma \nu}^{(\vec{k})}$ é um vetor coluna consistindo dos coeficientes $\left\{c_{\sigma \nu, i \alpha}^{(\vec{k})}\right\}$. A hamiltoniana $H_{\sigma}^{(\vec{k})} \mathrm{e}$ matriz de overlap $S^{(\vec{k})}$ são dadas por:

$$
\begin{aligned}
H_{\sigma, i \alpha j \beta}^{(\vec{k})} & =\sum_{n} e^{i \vec{k} \cdot \vec{R}_{n}} h_{\sigma, i \alpha j \beta, \vec{R}_{n}}, \\
S_{i \alpha j \beta}^{(\vec{k})} & =\sum_{n} e^{i \vec{k} \cdot \vec{R}_{n}} s_{i \alpha j \beta, \vec{R}_{n}},
\end{aligned}
$$

onde $h_{\sigma, i \alpha j \beta, \vec{R}_{n}}$ e $s_{i \alpha j \beta, \vec{R}_{n}}$ são os elementos de matriz da hamiltoniana e overlap, entre duas funções base $\phi_{i \alpha}\left(\vec{r}-\tau_{i}\right)$ e $\phi_{j \beta}\left(\vec{r}-\tau_{i}-\vec{R}_{n}\right)$, respectivamente. A matriz de overlap surge nas equações acima pois utilizamos um conjunto de orbitais base não-ortogonais.

A região central $C$, é composta pelas regiões $L_{0}, C_{0}$ e $R_{0}$, como na figura 3.2-b. Essa extensão da região central, $C_{0}$, é feita para que a relaxação da estrutura eletrônica nas interfaces entre os eletrodos $L_{0}$ e $R_{0}$ com a região central $C_{0}$ seja permitida. Além disso, são impostas duas condições necessárias para a continuação do desenvolvimento do formalismo:

(1) Existe overlap somente entre orbitais base $\phi_{i}$, localizados na região $C_{0}$ com os orbitais em $L_{0}$ e $R_{0}$. Este overlap não é permitido entre células não vizinhas a $C_{0}$, por exemplo, $C_{0} \operatorname{com} L_{1}$;

(2) Não existe overlap entre os orbitais base $\phi_{i}$, localizados nas regiões $L_{i}\left(R_{i}\right)$ com os orbitais nas células não vizinhas a esses, ou seja, o overlap entre $L_{i}\left(R_{i}\right)$ e $L_{i+2}\left(R_{i+2}\right)$ ou $L_{i-2}\left(R_{i-2}\right)$ são zero;

Na implementação realizada, as funções base são orbitais localizados no espaço real. Consequentemente, uma vez localizado esses orbitais, as duas condições anteriores, (1) e (2), podem sempre ser satisfeitas ajustando-se somente o tamanho das células $L_{i}$ e $R_{i}$. A matriz 
hamiltoniana da equação (3.21) pode ser escrita em uma forma bloco-tridiagonal como:

$$
H_{\sigma}^{\vec{k}}=\left(\begin{array}{ccccc}
\ddots & \ddots & & & 0 \\
\ddots & H_{\sigma, L_{1}}^{\vec{k}} & H_{\sigma, L_{1} C}^{\vec{k}} & & \\
& H_{\sigma, C L_{1}}^{\vec{k}} & H_{\sigma, C}^{\vec{k}} & H_{\sigma, C R_{2}}^{\vec{k}} & \\
& & H_{\sigma, R_{1} C}^{\vec{k}} & H_{\sigma, R_{2}}^{\vec{k}} & \ddots \\
0 & & & \ddots & \ddots
\end{array}\right),
$$

onde $H_{\sigma, C}^{\vec{k}}, H_{\sigma, L_{1}}^{\vec{k}}$ e $H_{\sigma, R_{1}}^{\vec{k}}$ são as matrizes hamiltonianas da região central $C$, do eletrodo esquerdo $L$ e do eletrodo direito $R$, respectivamente. A dimensão dessas matrizes é igual ao número de orbitais em $C, L$ e $R$, nas regiões $C, L_{1}$ e $R_{1}$, respectivamente. Os outros blocos de matrizes na equação (3.24), são as hamiltonianas que conectam duas regiões, ou seja, $L \Leftrightarrow C$ e $C \Leftrightarrow R$. A matriz de overlap do sistema possui a mesma estrutura que a hamiltoniana.

A estrutura eletrônica do sistema em questão pode ser obtida resolvendo-se esse conjunto de equações, dependentes de $\vec{k}$ utilizando as funções de Green, onde a equação (3.21) pode ser reescrita como:

$$
G_{\sigma}^{(\vec{k})}(Z)\left(Z S^{(\vec{k})}-H_{\sigma}^{(\vec{k})}\right)=\mathbf{1},
$$

e fazendo uso da forma tridiagonal de $H$ e $S$, a função de Green para a região central pode ser escrita como:

$$
G_{\sigma, C}^{(\vec{k})}(Z)=\left[Z S_{C}^{(\vec{k})}-H_{\sigma, C}^{(\vec{k})}-\Sigma_{\sigma, L}^{(\vec{k})}(Z)-\Sigma_{\sigma, R}^{(\vec{k})}(Z)\right]^{-1}
$$

onde $\Sigma_{\sigma, L}^{(\vec{k})}(Z)$ e $\Sigma_{\sigma, R}^{(\vec{k})}(Z)$ são as auto-energias dos eletrodos, dadas por:

$$
\begin{aligned}
& \Sigma_{\sigma, L}^{(\vec{k})}(Z)=\left(Z S_{C L_{1}}^{(\vec{k})}-H_{\sigma, C L_{1}}^{(\vec{k})}\right) G_{\sigma, L}^{(\vec{k})}(Z)\left(Z S_{L_{1} C}^{(\vec{k})}-H_{\sigma, L_{1} C}^{(\vec{k})}\right), \\
& \Sigma_{\sigma, R}^{(\vec{k})}(Z)=\left(Z S_{C R_{1}}^{(\vec{k})}-H_{\sigma, C R_{1}}^{(\vec{k})}\right) G_{\sigma, R}^{(\vec{k})}(Z)\left(Z S_{R_{1} C}^{(\vec{k})}-H_{\sigma, R_{1} C}^{(\vec{k})}\right),
\end{aligned}
$$

onde $G_{\sigma, L}^{(\vec{k})}(Z)$ e $G_{\sigma, R}^{(\vec{k})}(Z)$ são as funções de Green de superfície dos eletrodos esquerdo e direito, respectivamente. As funções de Green de superfície são calculadas utilizando um processo recursivo $[55,56]$. As matrizes de acoplamento $\Gamma$ do sistema, que permitem calcular a função de transmissão e outras grandezas importantes como a corrente, são dadas por:

$$
\Gamma_{\sigma, L}^{(\vec{k})}(Z)=i\left(\Sigma_{\sigma, L}^{(\vec{k})}(Z)-\left(\Sigma_{\sigma, L}^{(\vec{k})}(Z)\right)^{\dagger}\right)
$$




\subsubsection{Matriz densidade do sistema e grandezas físicas observáveis}

A matriz densidade do sistema pode ser calculada integrando a função de Green Lesser do sistema:

$$
\rho^{(\vec{k})}=\frac{1}{2 \pi i} \int_{-\infty}^{+\infty} G^{<(\vec{k})}(E) d E,
$$

onde $G^{<(\vec{k})}$ é dada por:

$$
G^{<(\vec{k})}(E)=G^{R(\vec{k})} \Sigma^{<(\vec{k})} G^{A(\vec{k})},
$$

onde $G^{R}$ e $G^{A}$ são as funções de Green retardada e avançada. $\Sigma^{<(\vec{k})}$ é dada por:

$$
\Sigma^{<(\vec{k})}(E)=i\left[\Gamma_{L}^{(\vec{k})} f\left(E-\mu_{L}\right)+\Gamma_{R}^{(\vec{k})} f\left(E-\mu_{R}\right)\right]
$$

onde $\Gamma$ é dada por 3.29. Combinando as equações (3.31) com a equação (3.32) temos:

$$
G^{<(\vec{k})}(E)=i G^{R(\vec{k})}\left[\Gamma_{L}^{(\vec{k})} f\left(E-\mu_{L}\right)+\Gamma_{R}^{(\vec{k})} f\left(E-\mu_{R}\right)\right] G^{A(\vec{k})} .
$$

Retornando o valor acima na integral da matriz densidade (3.30), temos:

$$
\rho^{(\vec{k})}=\frac{1}{2 \pi} \int_{-\infty}^{+\infty} G^{R(\vec{k})}\left[\Gamma_{L}^{(\vec{k})} f\left(E-\mu_{L}\right)+\Gamma_{R}^{(\vec{k})} f\left(E-\mu_{R}\right)\right] G^{A(\vec{k})} d E
$$

Para resolver esta equação integral, existem duas situações físicas diferentes que podemos estudar:

(1) O sistema está em equilíbrio, ou seja, os potenciais químicos dos dois contatos (eletrodos) são iguais, $f\left(E-\mu_{L}\right)=f\left(E-\mu_{R}\right)=f(E-\mu)$;

(2) O sistema está fora do equilíbrio, onde devido a presença de um bias externo nos eletrodos, os potenciais químicos destes são diferentes. Neste regime, existe uma corrente fluindo pelo dispositivo; 


\subsubsection{Matriz densidade no equilíbrio}

Na situação de equilíbrio, onde os contatos possuem o mesmo potencial químico, podemos simplificar a expressão (3.33) da seguinte maneira:

$$
\begin{array}{r}
G^{<(\vec{k})}(E)=i G^{R(\vec{k})}\left[\Gamma_{L}^{(\vec{k})} f(E-\mu)+\Gamma_{R}^{(\vec{k})} f(E-\mu)\right] G^{A(\vec{k})} \\
G^{<(\vec{k})}(E)=i G^{R(\vec{k})}\left[\Gamma_{L}^{(\vec{k})}+\Gamma_{R}^{(\vec{k})}\right] G^{A(\vec{k})} f(E-\mu)
\end{array}
$$

como $\Gamma_{L / R}^{(\vec{k})}=i\left[\Sigma_{L / R}^{(\vec{k})}-\Sigma_{L / R}^{(\vec{k}) \dagger}\right]$, o termo $\Gamma_{L}^{(\vec{k})}+\Gamma_{R}^{(\vec{k})}$ da equação (3.35) pode ser escrito como:

$$
\begin{aligned}
\Gamma_{L}^{(\vec{k})}+\Gamma_{R}^{(\vec{k})} & =i\left[\Sigma_{L}^{(\vec{k})}-\Sigma_{L}^{(\vec{k}) \dagger}+\Sigma_{R}^{(\vec{k})}-\Sigma_{R}^{(\vec{k}) \dagger}\right] \\
& =i\left[\left(-E S_{M}+H_{M}+\Sigma_{L}^{(\vec{k})}+\Sigma_{R}^{(\vec{k})}\right)-\left(-E S_{M}^{\dagger}+H_{M}^{\dagger}+\Sigma_{L}^{(\vec{k}) \dagger}+\Sigma_{R}^{(\vec{k}) \dagger}\right)\right] \\
& =i\left[\left(G^{A(\vec{k})}\right)^{-1}-\left(G^{R(\vec{k})}\right)^{-1}\right] .
\end{aligned}
$$

Substituindo $\Gamma_{L}^{(\vec{k})}+\Gamma_{R}^{(\vec{k})}$ em (3.35), temos

$$
\begin{array}{r}
G^{<(\vec{k})}(E)=i G^{R(\vec{k})} i\left[\left(G^{A(\vec{k})}\right)^{-1}-\left(G^{R(\vec{k})}\right)^{-1}\right] G^{A(\vec{k})} \\
G^{<(\vec{k})}(E)=-\left[G^{R(\vec{R})}-G^{A(\vec{k})}\right]
\end{array}
$$

retornando $G^{<(\vec{k})}$ dado pela equação (3.36) em (3.30), temos:

$$
\rho^{(\vec{k})}=\frac{i}{2 \pi} \int_{-\infty}^{+\infty}\left[G^{R(\vec{k})}(E)-G^{A(\vec{k})}(E)\right] f(E-\mu) d E
$$

Para um grande range do espectro de energia, é exigido um grande esforço computacional para resolver essa integral de maneira acurada no espaço real. Um resultado equivalente pode ser obtido integrando-se ao longo de um certo contorno no plano imaginário. Mas ainda existe um problema. A função de Green retardada $G^{R}$ é analítica acima do eixo real (na parte superior do plano complexo), porém, é não analítica abaixo do eixo complexo. O mesmo acontece com a função de Green avançada, que é analítica abaixo, e não analítica acima do eixo real. Para 
resolver isso, utilizamos a seguinte identidade ${ }^{2}$ :

$$
\begin{array}{r}
\int_{-\infty}^{+\infty}\left[G^{R(\vec{k})}(E)-G^{A(\vec{k})}(E)\right] f(E-\mu) d E= \\
\int_{-\infty}^{+\infty} G^{R(\vec{k})}(E) f(E-\mu) d E-\int_{-\infty}^{+\infty} G^{A(\vec{k})}(E) f(E-\mu) d E= \\
\int_{-\infty}^{+\infty} G^{R(\vec{k})}(E) f(E-\mu) d E-\left[\int_{-\infty}^{+\infty} G^{R(\vec{k})}(E) f(E-\mu) d E\right]^{\dagger}
\end{array}
$$

Dessa maneira, resta resolver somente uma integral do tipo:

$$
\int_{-\infty}^{+\infty} G^{R}(E) f(E-\mu) d E,
$$

onde o índice relacionado ao vetor de onda $\vec{k}$ foi omitido para simplificar a notação. Utilizando as propriedades da analiticidade da função de Green retardada na parte superior do plano complexo, essa integral pode ser resolvida como:

$$
\begin{gathered}
\int_{-\infty}^{+\infty} G^{R}(E) f(E-\mu) d E= \\
-\int_{C} G^{R}(z) f(z-\mu) d z-2 \pi i k_{B} T \sum_{k=1}^{n} G^{R}\left(z_{k}\right)
\end{gathered}
$$

onde

$$
z_{k}=i(2 k+1) \pi k_{B} T
$$

são os pólos da função de distribuição de Fermi-Dirac. O primeiro termo da equação (3.41) é integrado em um contorno $C$ no plano imaginário. A figura 3.3 mostra um exemplo dos pontos deste contorno. Para obter os pontos de integração e os correspondentes pesos ao longo do contorno, foi utilizado o método de quadratura Gaussiana, baseado na integração de GaussLegendre [57].

Uma vez obtida a matriz densidade para cada vetor de onda $\vec{k}$, para obter a matriz densidade total do sistema, basta realizar a integral abaixo:

$$
\rho=\frac{1}{(2 \pi)^{2}} \int \rho(\vec{k}) d^{2} \vec{k}
$$

\footnotetext{
${ }^{2}$ Utilizamos o fato aqui que $G^{A}=G^{R \dagger}$
} 


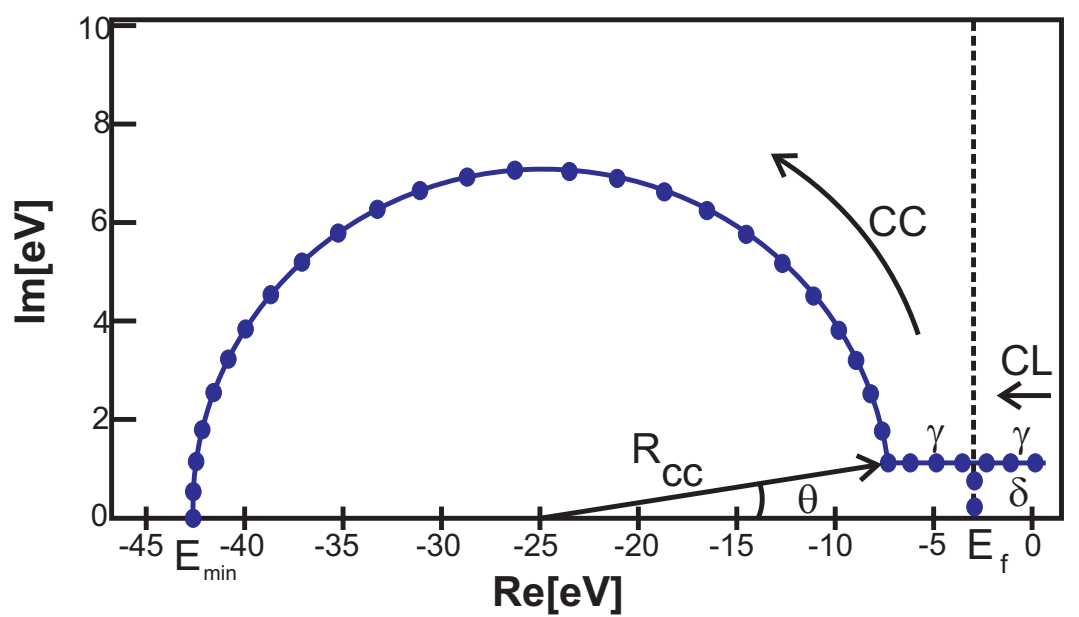

Figura 3.3: Contorno fechado para integração no equilíbrio. Os termos $\gamma, \theta, R C, C C, C L$ são parâmetros utilizados no método de integração de Gauss-Legengre [57].

\subsubsection{Matriz densidade fora do equilíbrio}

Até agora, discutimos o esquema para calcular a matriz densidade de um sistema aberto na condição de equilíbrio. Para gerar um fluxo de corrente finito no dispositivo, devemos considerar o sistema na presença de uma voltagem de bias externo, que gera um estado fora do equilíbrio. Os potenciais químicos dos eletrodos serão agora diferentes um do outro. Nesse estado fora do equilíbrio, existem um efeito importante que é o deslocamento dos potenciais químicos dos eletrodos devido à presença da voltagem de bias. Isso gera uma mudança no potencial de Hartree do dispositivo.

O tratamento do potencial de Hartree nesta tese, foi abordado de duas maneiras. (1) Utilização da transformada de fourier (FFT) convencional para resolver a equação de Poisson do sistema, utilizando condições periódicas de contorno. Após a obtenção da solução, é aplicado um shift rígido no potencial dos eletrodos juntamente com uma rampa de potencial na região de espalhamento. Um esquema dessas condições de contorno é apresentado na figura 3.4-a-2: (2) Uma outra abordagem, foi a solução da equação de Poisson no espaço real, utilizando uma técnica de Multigrid que será discutida em mais detalhes na seção 3.3 e no apêndice A. As condições de contorno mostradas na figura $3.4-b^{3}$ podem ser escritas como:

$$
\begin{gathered}
\left.V_{H}(z)\right|_{z=0}=V_{H}^{\text {Elec-L }}\left(z=z_{L}\right)+U_{\text {Bias } L} \\
\left.V_{H}(z)\right|_{z=z_{f}}=V_{H}^{\text {Elec-R }}\left(z=z_{R}\right)+U_{\text {Bias } L}
\end{gathered}
$$

\footnotetext{
${ }^{3}$ Utilizando este método, não é preciso aplicar uma rampa de potencial no sistema nem realizar um shift no potencial dos eletrodos.
} 

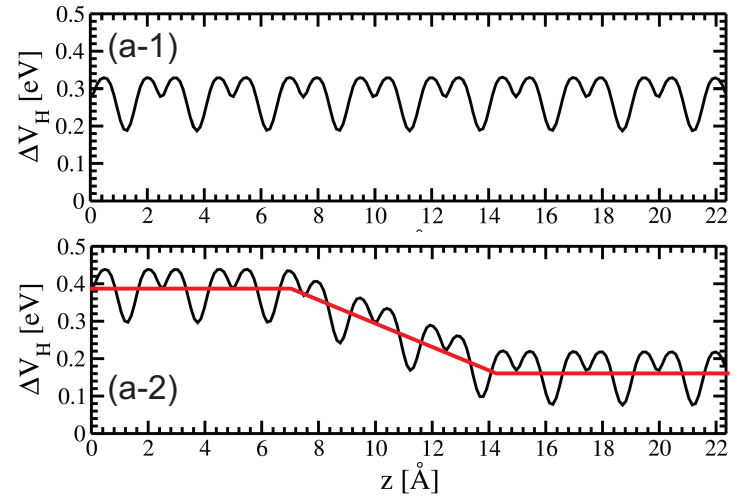

(b)

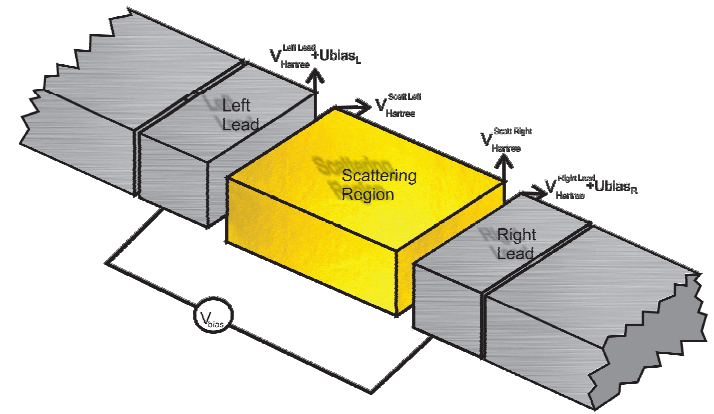

Figura 3.4: Representação esquemática das condições de contorno para os cálculos fora do equilíbrio. (a-1) potencial de Hartree médio para o sistema pristino. (a-2) Potencial de Hartree do sistema (a-1) sujeita à rampa de potencial. (b) Esquema para as condições de contorno no cálculo multigrid.

Considerando os efeitos e condições de contorno descritas anteriormente, a matriz densidade do sistema pode ser calculada na condição fora do equilíbrio. Para isso, vamos considerar novamente as equações (3.30) e (3.33) que fornecem a matriz densidade e a função de Green Lesser, respectivamente. Na condição fora do equilíbrio, os potenciais químicos dos eletrodos são diferentes um do outro, $f\left(E-\mu_{L}\right) \neq f\left(E-\mu_{R}\right)$. Somando e subtraindo o termo $i G^{R(\vec{k})} \Gamma_{R}^{(\vec{k})} G^{A(\vec{k})} f\left(E-\mu_{L}\right)$ na função de Green Lesser (3.33), temos:

$$
\begin{aligned}
G^{<(\vec{k})}(E) & =i G^{R(\vec{k})}\left(\Gamma_{L}^{(\vec{k})}+\Gamma_{R}^{(\vec{k})}\right) G^{A(\vec{k})} f\left(E-\mu_{L}\right) \\
& +i G^{R(\vec{k})} \Gamma_{R}^{(\vec{k})} G^{A(\vec{k})}\left(f\left(E-\mu_{R}\right)-f\left(E-\mu_{L}\right)\right)
\end{aligned}
$$

Utilizando a equação (3.36) para $\Gamma_{L}^{(\vec{k})}+\Gamma_{R}^{(\vec{k})}$, temos:

$$
\begin{aligned}
G^{<(\vec{k})}(E) & =-\left[G^{R(\vec{k})}-G^{A(\vec{k})}\right] f\left(E-\mu_{L}\right) \\
& +i G^{R(\vec{k})} \Gamma_{R}^{(\vec{k})} G^{A(\vec{k})}\left(f\left(E-\mu_{R}\right)-f\left(E-\mu_{L}\right)\right)
\end{aligned}
$$


Substituindo (3.45) na equação (3.30), temos:

$$
\begin{aligned}
\rho^{(\vec{k})} & =\frac{i}{2 \pi} \int_{-\infty}^{+\infty}\left[G^{R(\vec{k})}-G^{A(\vec{k})}\right] f\left(E-\mu_{L}\right) d E \\
& +\frac{1}{2 \pi} \int_{-\infty}^{+\infty} G^{R(\vec{k})} \Gamma_{R}^{(\vec{k})} G^{A(\vec{k})}\left(f\left(E-\mu_{R}\right)-f\left(E-\mu_{L}\right)\right) d E
\end{aligned}
$$

Dessa maneira, a matriz densidade do sistema pode ser escrita como a soma da parte no equilíbrio mais uma parte fora do equilíbrio, ou seja:

$$
\begin{gathered}
\rho^{(\vec{k})}=\rho_{e q}^{(\vec{k})}+\rho_{\text {neq }}^{(\vec{k})}, \\
\rho_{e q}^{(\vec{k})}=\frac{i}{2 \pi} \int_{-\infty}^{+\infty}\left[G^{R(\vec{k})}-G^{A(\vec{k})}\right] f\left(E-\mu_{L}\right) d E \\
\rho_{\text {neq }}^{(\vec{k})}=\frac{1}{2 \pi} \int_{-\infty}^{+\infty} G^{R(\vec{k})} \Gamma_{R}^{(\vec{k})} G^{A(\vec{k})}\left(f\left(E-\mu_{R}\right)-f\left(E-\mu_{L}\right)\right) d E,
\end{gathered}
$$

A integral da equação (3.48) pode ser resolvida da mesma maneira que foi feita anteriormente para o caso do equilíbrio, utilizando uma continuação analítica no plano complexo. A integral da equação (3.49) é realizada diretamente no eixo real, entre os dois potenciais químicos dos eletrodos. A figura 3.5, mostra um esquema do contorno utilizado para essa integração.

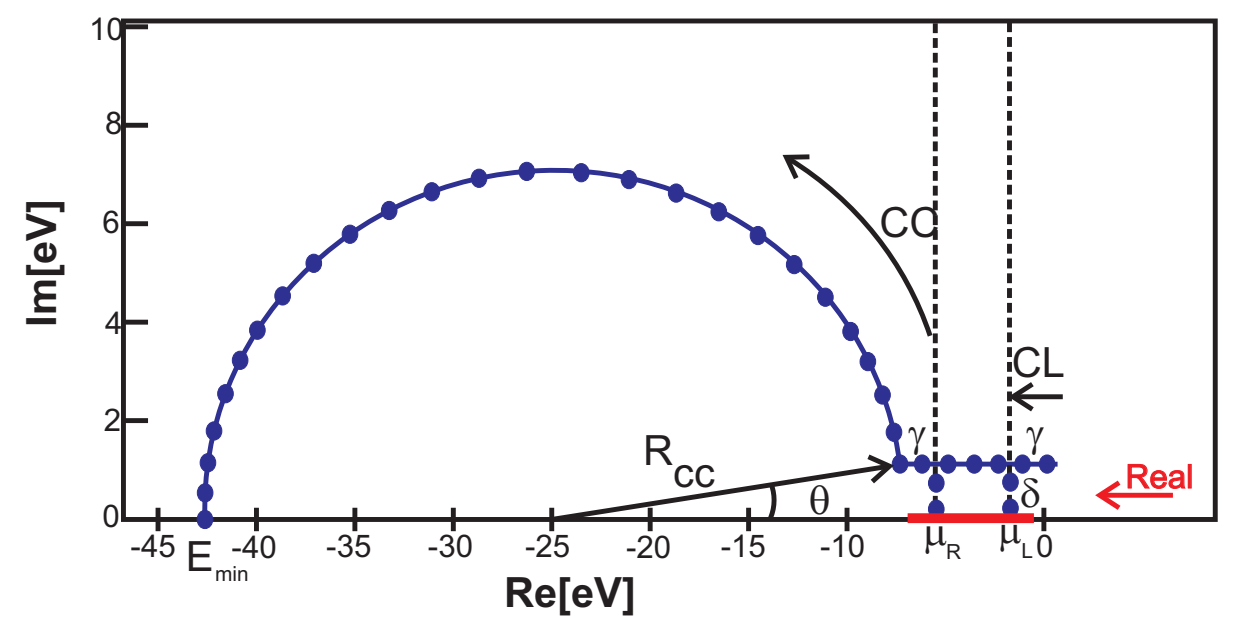

Figura 3.5: Contorno fechado para integração fora do equilíbrio. Os termos $\gamma, \theta, R C, C C, C L$ são parâmetros utilizados no método de integração de Gauss-Legendre [57]. 


\subsubsection{Análise e grandezas físicas}

Uma vez obtida a convergência, as funções de Green do sistema e a densidade eletrônica fornecem todas as informações físicas sobre o sistema da seguinte maneira. Em um primeiro passo, podemos calcular as funções espectrais do sistema como:

$$
A^{(\vec{k})}(E)=A_{L}^{(\vec{k})}(E)+A_{R}^{(\vec{k})}(E)
$$

onde

$$
A_{L / R}^{(\vec{k})}(E)=\frac{1}{2 \pi} G^{R(\vec{k})} \Gamma_{L / R}^{(\vec{k})} G^{A(\vec{k})}
$$

$\mathrm{e}$

$$
\Gamma_{L / R}^{(\vec{k})}(E)=i\left[\Sigma_{L / R}^{(\vec{k})}-\Sigma_{L / R}^{(\vec{k}) \dagger}\right]
$$

A partir dessas grandezas podemos calcular a densidade de estados (DOS) a partir do traço das funções espectrais vezes a matriz de overlap:

$$
\operatorname{DOS}(E, V)=\int_{B Z} w_{(\vec{k})} d \vec{k} \operatorname{Tr}\left[A^{(\vec{k})}(E, V) S^{(\vec{k})}\right]
$$

onde $w_{(\vec{k})}$ é um fator de peso para cada vetor da rede $\vec{k}$. Por sua vez, a função de transmissão, que fornece a probabilidade de um elétron ser transmitido em cada estado de energia pode ser obtido a partir da seguinte equação:

$$
T(E, V)=\int_{B Z} w_{(\vec{k})} d \vec{k} T^{(\vec{k})}(E, V)
$$

onde

$$
T^{(\vec{k})}(E, V)=\operatorname{Tr}\left[\Gamma_{L}^{(\vec{k})} G^{R(\vec{k})} \Gamma_{R}^{(\vec{k})} G^{A(\vec{k})}\right]
$$

A corrente do sistema pode ser calculada como:

$$
I(E, V)=\frac{2 e}{h} \int_{-\infty}^{+\infty} T(E, V)\left[f\left(E-\mu_{L}\right)-f\left(E-\mu_{R}\right)\right] d E,
$$

onde $e$ é a carga do elétron e $h$ é a constante de Planck.

Todas as variáveis nas equações $(3.50,3.51,3.52,3.53,3.54,3.55)$ são funções da energia $E$ 
e da voltagem de bias $V$. Podemos ainda generalizar essas grandezas se incluirmos o grau de liberdade de spin. Dessa maneira, as funções de Green, matriz densidade e matrizes gamma são obtidas para cada componente de spin. De posse dessas grandezas, a transmitância para cada spin é dada por:

$$
T_{\sigma}^{(\vec{k})}(E, V)=\operatorname{Tr}\left[\Gamma_{L, \sigma}^{(\vec{k})} G_{\sigma}^{R(\vec{k})} \Gamma_{R, \sigma}^{(\vec{k})} G_{\sigma}^{A(\vec{k})}\right]
$$

onde $\sigma$ identifica o índice do spin.

A corrente do sistema $I$ pode ser calculada para cada índice de spin $\sigma$ utilizando a fórmula de Meir-Wingreen [58], como:

$$
I_{\sigma}(E, V)=\frac{e}{h} \int_{-\infty}^{+\infty} T_{\sigma}(E, V)\left[f\left(E-\mu_{L}\right)-f\left(E-\mu_{R}\right)\right] d E,
$$

onde a corrente total é dada pela soma das correntes para cada componente de spin.

\subsubsection{Representação esquemática do ciclo auto-consistente}

A metodologia de funções de Green acoplada com a teoria do funcional da densidade descrita anteriormente, foi implementada no código SIESTA - denominada de TRANSAMPA. Todo o cálculo de transporte é realizado de maneira auto-consistente. Na figura 3.6, apresentamos um esquema do ciclo auto-consistente utilizado no código TRANSAMPA. Primeiramente é realizado um cálculo de eletrodo, onde são obtidas as funções de Green de superfície, autoenergias e matrizes Gamma. Após o cálculo de eletrodo, inicia-se o cálculo das propriedades de transporte. O código SIESTA constrói as matrizes hamiltoniana e overlap do sistema e esta informação, juntamente, com os resultados para os eletrodos são passadas ao código TRANSAMPA, que calcula agora a nova matriz densidade para o sistema aberto utilizando o método de funções de Green. Após isso, é realizada uma verificação do critério de convergência. Se a convergência não foi obtida, a matriz densidade é passada de volta ao código SIESTA, que irá gerar uma nova hamiltoniana e overlap, e o ciclo auto-consistente se inicia novamente. Se a convergência for obtida, são calculadas as quantidades físicas, como densidade de estados (DOS), transmitância $(T(E))$ e corrente. 


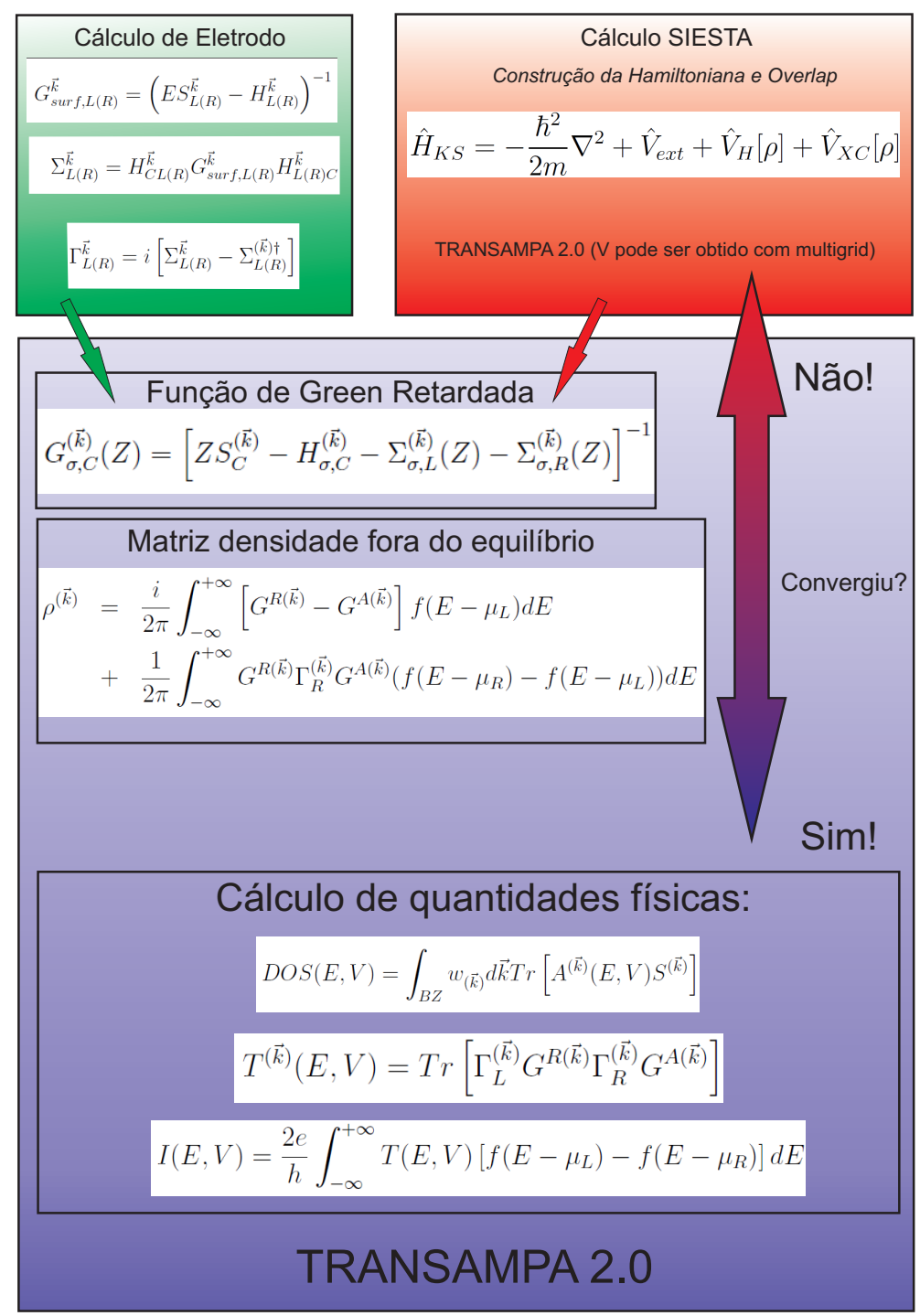

Figura 3.6: Ciclo auto-consistente do código TRANSAMPA2.0.

\subsection{Implementação de multigrid}

Dentro do método de Kohn-Sham descrito na seção 3.1, é necessário a solução da equação de Poisson do sistema para a obtenção do potencial de Hartree, $V_{H}[\rho]$. Para isso é utilizado um método de transformada de Fourier (FFT) da densidade. Entretanto, com a utilização da FFT são impostas condições periódicas de contorno no problema, restringindo dessa forma o número de problemas que podem ser tratados, por exemplo, o transporte eletrônico na presença de um gate de comprimento finito, com controle independente dos valores de top/back gate. Desse modo, é necessário a utilização de novo método para a solução da equação de Poisson sem o vínculo do uso de condições periódicas de contorno. Um método amplamente utilizados 
em problemas diferenciais no espaço real são as técnicas de multigrid.

Métodos de multigrid são um conjunto de algoritmos para solução de equações diferenciais utilizando uma hierarquia de discretizações. A aplicação típica para o multigrid é na solução de equações diferenciais parciais elípticas em duas ou mais direções. O multigrid pode ser aplicado junto com qualquer técnica comum de discretização. Nesses casos, o multigrid está entre as soluções mais rápidas conhecidas hoje em dia. Em contraste com outros métodos, o multigrid pode ser aplicado com condições de contorno arbitrárias e não depende da separabilidade da equação diferencial em questão. Existem muitas variações de algoritmos de multigrid. A característica mais representativa e comum a todos é que existe uma hierarquia de discretizações, ou seja, uma hierarquia de malhas.

Em um método multigrid as componentes mais importantes são: Suavização - redução de erros de alta frequência, por exemplo, através de algumas iterações do método de Gauss-Seidel ou Jacobi; Restrição - passagem do erro residual para uma malha mais grossa; Correção interpolação de valores do resíduo calculados na malha mais grossa para uma malha mais fina.

Nesta seção, iremos somente fornecer algumas informações pertinentes sobre como é utilizado o método de multigrid implementado no código SIESTA para a solução da equação de Poisson. Forneceremos alguns detalhes sobre os parâmetros de entrada para a simulação e determinação do grid. Apresentaremos também alguns testes da implementação em sistemas de interesse que iremos estudar no restante da tese.

Uma descrição detalhada de todas as componentes do método de multigrid e como são agrupadas para a solução de um problema diferencial estão no apêndice A.

\subsubsection{Detalhes da implementação de multigrid}

Os passos realizados pelo método de multigrid implementado no código SIESTA para a obtenção da solução da equação de Poisson podem ser resumidos como:

1 Leitura dos Parâmetros: Nesta etapa, o código SIESTA realiza a leitura a partir do arquivo de entrada dos parâmetros para os ciclos multigrid. Os parâmetros são:

a- Número dos pontos do grid nas direções $x, y$ e $z$;

b- Tipo das condições de contorno nos planos que delimitam o sistema - $X 1-X 2$, $Y 1-Y 2$ e $Z 1-Z 2$ 
c- Condição se realiza cálculos de gate - Se .true., lê em quais planos é aplicado o campo, o valor do potencial aplicado em cada um, e a região onde inicia e termina o gate;

d- Leitura do tipo do ciclo: $\mathrm{KCICLO}=1$ ciclos $\mathrm{V},=2$ ciclos $\mathrm{W}$;

e- Leitura do número de pré-relaxações, pós-relaxações e número de ciclos realizados (Os valores são definidos através de testes de convergência);

f- Tipo de interpolação realizada para passagem das informações entre os grids: $=1$ interpolação linear; = 3 interpolação cúbica;

g- Leitura da condição se realiza ciclo FULL-MULTIGRID;

h- Leitura dos parâmetros que definem os coarse grids. A determinação dos coarse grids é realizada através da equação: $n x k(k)=i x p \times 2^{(k+i e x-n g r i d)}+1$, onde $k$ é o índice do coarse grid, $n x k(k)$ é o número de pontos no coarse grid $k$, ixp e $i e x$ são parâmetros que definem o grid e ngrid é o número de coarse grids.

2 Início do ciclo: O código SIESTA envia a matriz densidade nos pontos do grid, $\rho_{i, j, k}$, para a subrotina que realiza o multigrid;

3 Cálculo da solução: Após a leitura dos parâmetros de $a-h$ descritos anteriormente e da leitura da matriz densidade do problema a subrotina implementada realiza os ciclos multigrid como descrito no apêndice A, onde o método de relaxação do erro utilizado é o RED-BLACK GAUSS SEIDEL descrito na seção do apêndice A.2.3;

4 Após ser realizados todos os passos do ciclo multigrid, a solução final é retornada para o SIESTA;

\subsubsection{Testes da implementação de multigrid}

Nesta seção, apresentaremos os resultados dos testes para a implementação da solução da equação de Poisson através da técnica de multigrid apresentada brevemente na seção 3.3.1 e em detalhes no apêndice A.

Para testar a implementação do método de multigrid, realizamos cálculos com sistemas conhecidos e de interesse para a tese, utilizando o SIESTA na versão com FFT e cálculos com 
a versão multigrid, SIESTA-MG. Os sistemas escolhidos foram as nanofitas zig-zag de grafeno $(3,0)$, o grafeno e as bicamadas de grafeno.

Para os cálculos SIESTA-FFT utilizamos uma base DZP, com um energy-shift de $0.1 \mathrm{eV}$. O mesh-cutoff utilizado foi de 300 Ry. Para os cálculos com o SIESTA-MG, utilizamos os mesmos parâmetros descritos anteriormente, porém, o método multigrid implementado necessita de mais algumas informações, como já explicamos na seção 3.3.1. Utilizamos um ciclo de full-multigrid associado a ciclos $W$, o número de pré-relaxações e pós relaxações foi definido para 10 iterações.

\subsubsection{Nanofitas de Grafeno $(3,0)$}

Como testes para as nanofitas, realizamos cálculos com SIESTA-FFT ${ }^{4}$ e SIESTA-MG ${ }^{5}$. Na figura 3.7, apresentamos as densidades de estados e estrutura de bandas calculadas com os dois métodos citados anteriormente. Em (a) acoplamento ferromagnético entre os átomos de bordas opostas e (b) acoplamento anti-ferromagnético. Como podemos observar da figura
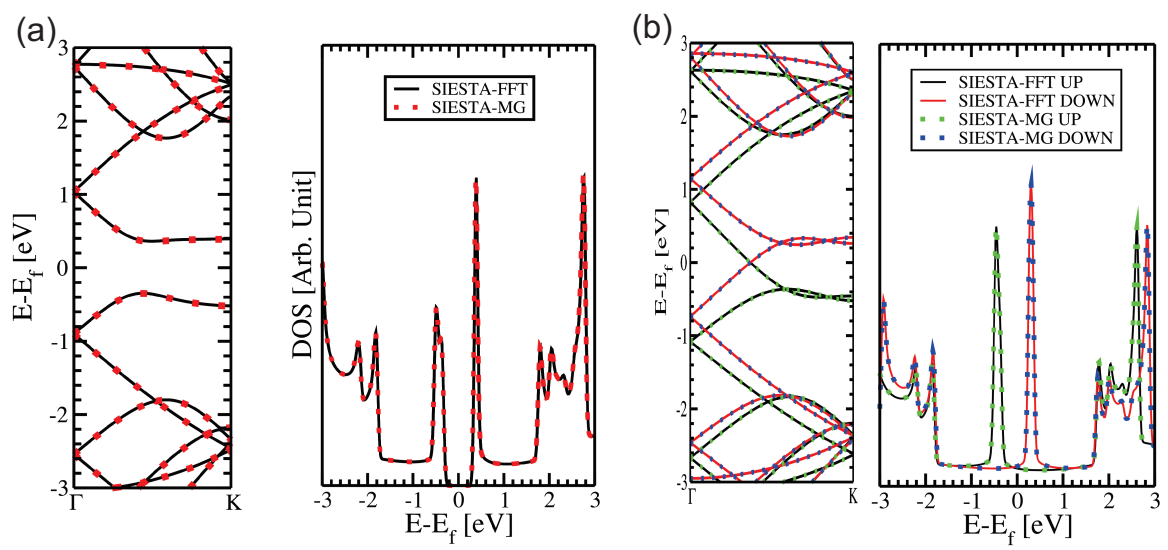

Figura 3.7: Estrutura de bandas e densidade de estados para a nanofita zig-zag $(3,0)$ nas configurações:(a) Acoplamento anti-ferromagnético entre as bordas e (b) Acoplamento ferromagnético. As linhas cheias são cálculos com o SIESTA-FFT e as pontilhadas com a implementação do Multigrid.

3.7, a técnica de multigrid para a solução da equação de Poisson reproduz com fidelidade as estruturas de bandas e densidades de estados para as nanofitas tanto nas configurações antiferromagnética quanto ferromagnética.

\footnotetext{
${ }^{4}$ Versão do siesta que utiliza transformada de Fourier para resolver a equação de Poisson.

${ }^{5}$ Versão do siesta que utiliza a nossa implementação do método de multigrid para a solução da equação de Poisson.
} 


\subsubsection{Grafeno e Bicamadas de Grafeno}

Nesta seção, apresentaremos os resultados dos testes para o SIESTA-FFT e SIESTA-MG, para as monocamadas e bicamadas de grafeno sem a presença de um campo elétrico. Apresentaremos, também, ainda como teste, os resultados para as bicamadas de grafeno na presença de um campo elétrico perpendicular, calculados com o SIESTA-FFT e com o SIESTA-MG, mostrando que através da técnica de multigrid para a solução da equação de Poisson, podemos gerar um campo elétrico simplesmente configurando as condições de contorno do sistema como sendo um capacitor de placas paralelas infinito ${ }^{6}$.

Na figura 3.8, mostramos as densidades de estados e estruturas de bandas para os cálculos efetuados com o SIESTA-FFT e o SIESTA-MG para uma única camada de grafeno.
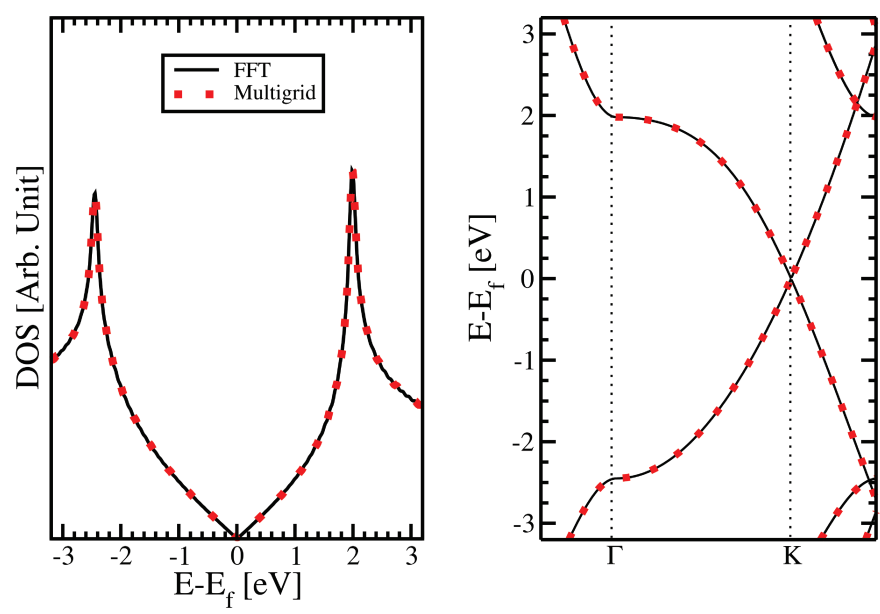

Figura 3.8: Densidade de estados e estrutura de bandas para a monocamada de grafeno. As linhas cheias são cálculos com o SIESTA-FFT e as pontilhadas com a implementação do Multigrid.

Como observamos na figura 3.8, novamente temos uma concordância exata entre os cálculos efetuados com o SIESTA-FFT e SIESTA-MG.

Na figura 3.9, os resultados para as bicamadas de grafeno. Novamente, observamos a exata concordância entre as duas técnicas.

Além da configuração da bicamada pristina, sabe-se da literatura $[6,7,59,60]$ que quando é aplicado um campo elétrico perpendicular à bicamada de grafeno existe uma abertura de um gap de energia. Com o SIESTA-FFT podemos aplicar esse campo elétrico externo simplesmente definindo no arquivo de input a direção do campo e a intensidade, em $V / \AA$, enquanto que no SIESTA-MG, precisamos simplesmente definir em qual contorno será aplicado as tensões. O

\footnotetext{
${ }^{6}$ Infinito aqui quer dizer que estamos resolvendo o problema com condições periódicas de contorno
} 

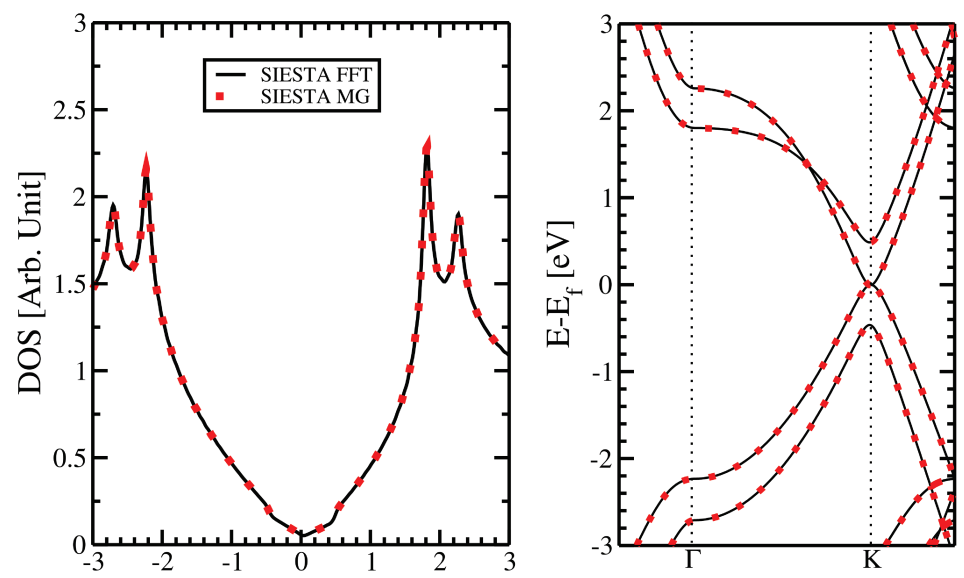

Figura 3.9: Densidade de estados e estrutura de bandas para a bicamada de grafeno. As linhas cheias são cálculos com o SIESTA-FFT e as pontilhadas com a implementação do Multigrid.

campo, aqui, foi aplicado no sistema inteiro. A figura 3.10, mostra o resultados para os cálculos com o SIESTA-FFT e o SIESTA-MG.

Mais um vez, observamos a concordância entre os dois métodos de cálculos. No capítulo 4, estudaremos detalhadamente um dispositivo constituído por uma bicamada de grafeno na presença de dois gates, onde através da técnica de multigrid será possível a utilização de gates com comprimentos finitos e o controle das voltages aplicadas podem ser sintonizadas de maneiras independentes. 
(a)
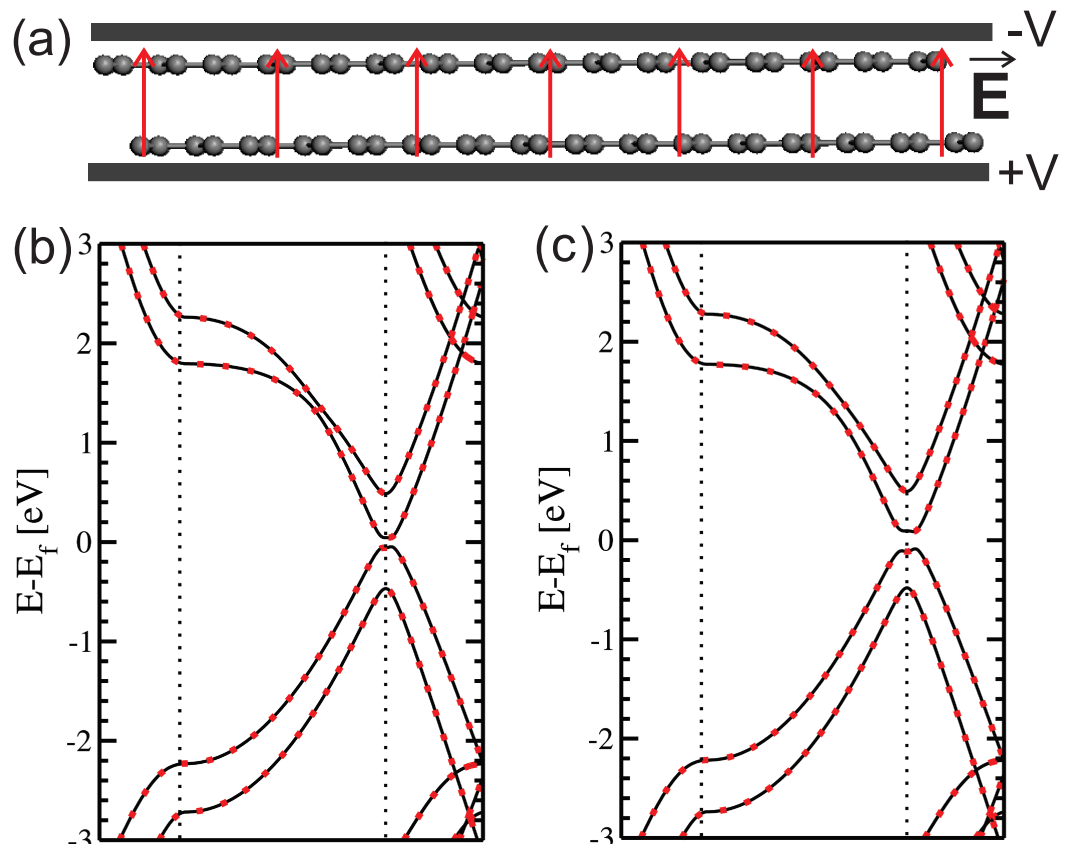

Figura 3.10: (a) Representação esquemática da bicamada de grafeno na presença do campo elétrico e/ou gate aplicado. O campo é aplicado no sistema inteiro, onde este é periódico em todas as direções. Estrutura de bandas para a bicamada na presença de um campo elétrico de intensidade (a) $0.1 \mathrm{~V} / \mathrm{A} e$ (b) $0.2 \mathrm{~V} / \AA$ A, calculadas com o SIESTA-FFT (linhas pretas contínuas) e SIESTA-MG (linhas pontilhadas vermelhas) 


\section{Capítulo 4}

\section{Nanotransistor de bicamada de grafeno}

Neste capítulo, apresentaremos as investigações feitas através de cálculos de primeiros princípios das propriedades de transporte eletrônico de um nano-dispositivo de bicamada de grafeno com gate duplo. Um estudo sistemático foi feito variando os potenciais de gate e de bias, em função do comprimento do gate, $L_{\text {gate }}$, e da temperatura. Mostraremos que a corrente apresenta um decaimento exponencial como função do comprimento do gate, característico de um regime de tunelamento. Como resultado, o sistema nunca atinge uma corrente nula. Discutiremos também, as condições para obtenção do ponto de neutralidade de carga (CNP) em um sistema livre de defeitos intrínsecos e dopagens extrínsecas. Os campos elétricos utilizados nas simulações são comparáveis com os campos elétricos de deslocamento nos experimentos, $1.0 \mathrm{~V} / \mathrm{nm}<|E|<5.0 \mathrm{~V} / \mathrm{nm}$. As simulações foram realizadas para temperatura ambiente e $4.5 \mathrm{~K}$. Os cálculos de transporte foram realizados utilizando-se o modelo de Landauer-Büttiker, com uma hamiltoniana gerada através de cálculos ab initio baseadas na Teoria do Funcional da Densidade (DFT) [37, 40,61], acoplado com o formalismo de Funções de Green fora do Equilíbrio (NEGF-DFT) [62-64], como descrito na seção 3.2. Para investigar os efeitos de gates finitos, implementamos um método no espaço real para solução da equação de Poisson através de técnicas de multigrid [65].

\subsection{Propriedades estruturais e eletrônicas da bicamada de grafeno}

Antes de discutirmos os resultados referente a bicamada grafeno como potencial nanotransistor, apresentaremos as propriedades eletrônicas e estruturais desse sistema. Na figura 4.1, mostramos uma bicamada de grafeno, que consiste em duas monocamadas de grafeno empil- 
Tabela 4.1: Energias de formação $(\mathrm{eV})$ e distâncias de equilíbrio $(\AA)$ da bicamada de grafeno.

\begin{tabular}{cc|cc}
\hline \hline LDA & & GGA+VDW & \\
\hline \hline$E_{f}(e V)$ & $d(\AA)$ & $E_{f}(e V)$ & $d(\AA)$ \\
\hline \hline-0.076 & 3.210 & -0.113 & 3.325 \\
\hline \hline
\end{tabular}

hadas uma sobre a outra de acordo com o empilhamento de Bernal, AB.
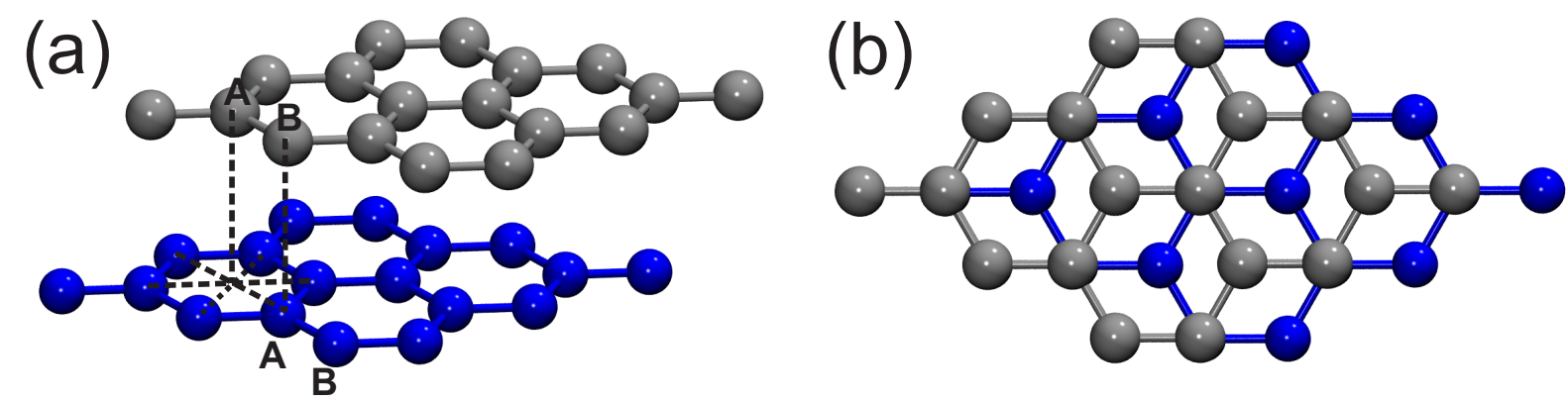

Figura 4.1: Visão esquemática da bicamada de grafeno com empilhamento de Bernal AB.

Realizamos cálculos de energia total, baseados na teoria do funcional da densidade, para o estudo das propriedades eletrônicas e estruturais. Foi utilizado o funcional de troca e correlação LDA [66] e GGA+VDW como feito por Grimme [67]. Todas as geometrias foram relaxadas até que as forças fossem menores que $0.02 \mathrm{eV} / \AA$ e o parâmetro de rede foi otimizado para o valor da monocamada de grafeno. Na tabela 4.1 mostramos os resultados para as energias de formação e distâncias de equilíbrio das bicamadas de grafeno. A energia de formação foi calculada utilizando a expressão:

$$
E_{f}=E_{\text {total }}(B G)-2 E_{\text {total }}(S G)
$$

onde $E_{\text {total }}(B G)$ é a energia total para a bicamada de grafeno e $E_{\text {total }}(S G)$ é a energia total para uma monocamada de grafeno(Utilizamos o grafeno como fonte dos átomos de carbono).

$\mathrm{Na}$ tabela 4.1, comparamos os dois tipos de funcionais utilizados, onde podemos observar que a utilização do GGA+vdW descreve com mais precisão a distância entre as camadas que é de $\approx 3.3 \AA$.

Na figura 4.2, apresentamos a estrutura de bandas de energia na região próxima ao nível de Fermi, para a bicamada de grafeno, utilizando as aproximações LDA e GGA+vdW. Podemos observar que não é possível notar nenhuma diferença significativa na estrutura eletrônica desse sistema quando comparamos as aproximações LDA e GGA+vdW. Dessa maneira, é justificável 

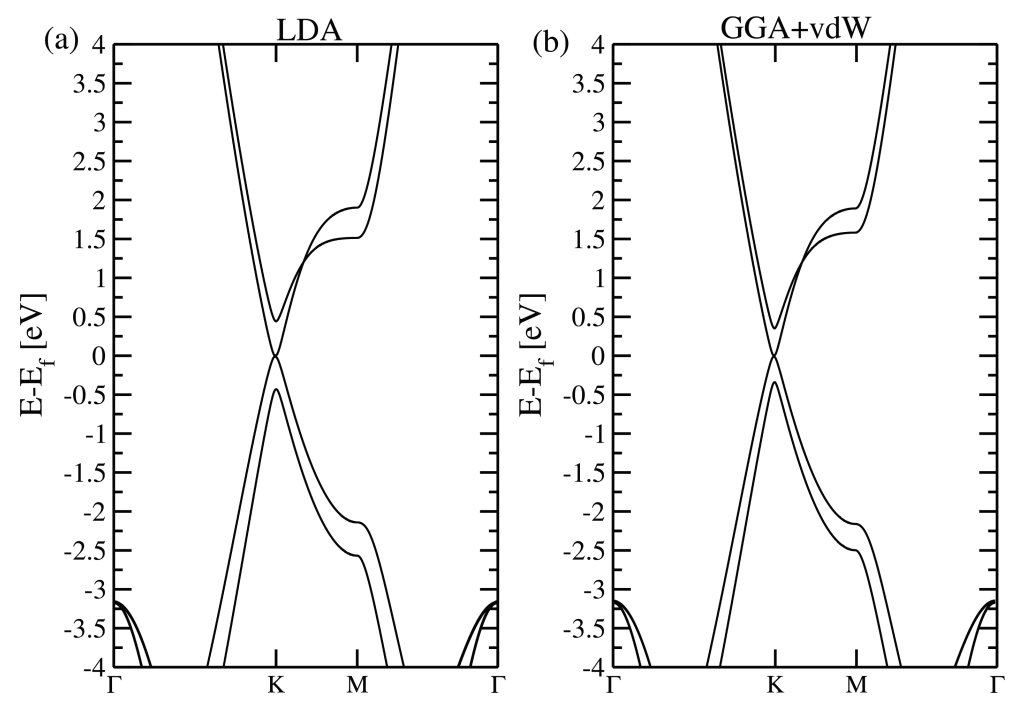

Figura 4.2: Estrutura de bandas para a bicamada de grafeno com as aproximações (a) LDA e (b) GGA+vdW.

a utilização da aproximação LDA para o estudo das propriedades eletrônicas e de transporte do sistema em questão ${ }^{1}$.

Uma das propriedades mais interessantes das bicamadas de grafeno, é a abertura de um gap de energia controlável através da aplicação de um campo elétrico. Na figura 4.3(a), apresentamos a estrutura eletrônica de uma bicamada de grafeno na presença de um campo elétrico externo perpendicular às camadas de intensidade $5 \mathrm{~V} / \mathrm{nm}$ - linha vermelha preenchida.

$\mathrm{Na}$ figura 4.3(b), apresentamos o comportamento do gap de energia induzido pelo campo elétrico externo. A linha vermelha corresponde ao gap induzido no ponto de alta simetria $K$ e a linha preta corresponde ao gap homo-lumo.

\subsection{Transistor de bicamada de grafeno}

Na figura 4.4, mostramos duas imagens experimentais de um transistor de bicamada de grafeno, onde em (a) temos um dispositivo suspenso, ou seja, sem a presença de um dielétrico entre os eletrodos de gate e o canal, e em (b) temos um dispositivo na presença de um dielétrico. O ponto importante, aqui, como podemos notar nas regiões indicadas pelas setas em vermelho, é que o eletrodo de gate utilizado possui dimensões finitas. Dessa maneira, o estudo das propriedades de transporte desse tipo sistema com o campo aplicado em todo o sistema $[36,68]$

\footnotetext{
${ }^{1} \mathrm{O}$ custo computacional da implementação de vdW nos cálculos de transporte é muito grande, sendo impraticável o estudo de sistemas com uma grande quantidade átomos.
} 

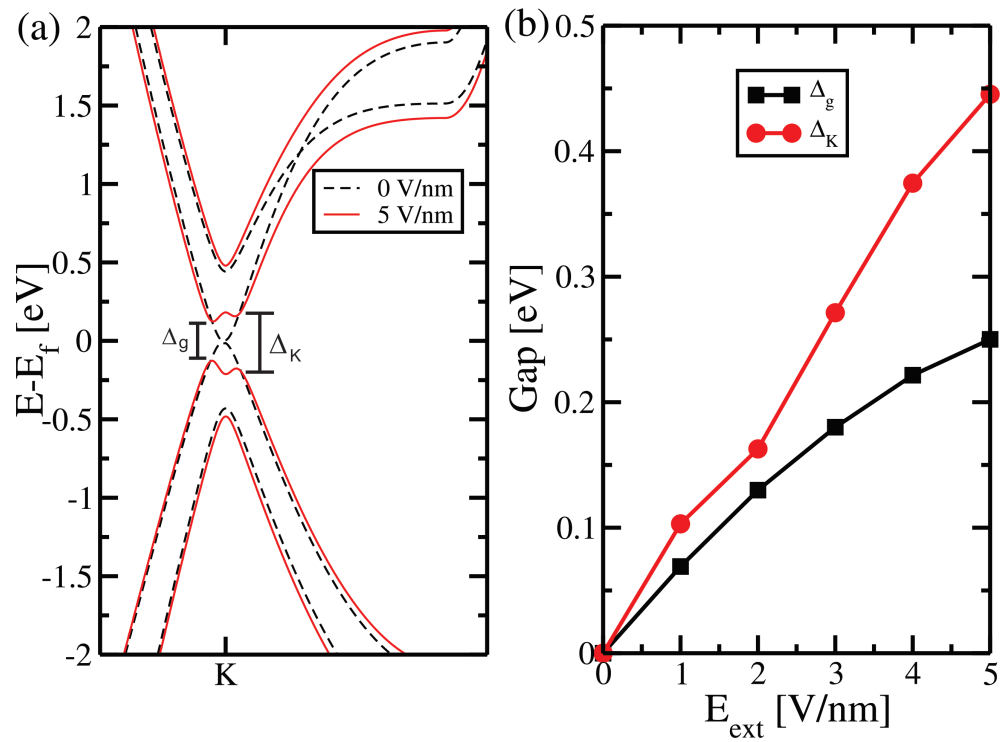

Figura 4.3: (a)Estrutura de bandas para uma bicamada de grafeno na presença de um campo elétrico de $5 \mathrm{~V} / \mathrm{nm}$, mostrando o gap no ponto $K, \Delta_{K}$, e o gap homo-lumo, $\Delta_{g}$. (b)Gap de energia em função do campo aplicado para a BG.

pode não representar de maneira realista os resultados observados experimentalmente.
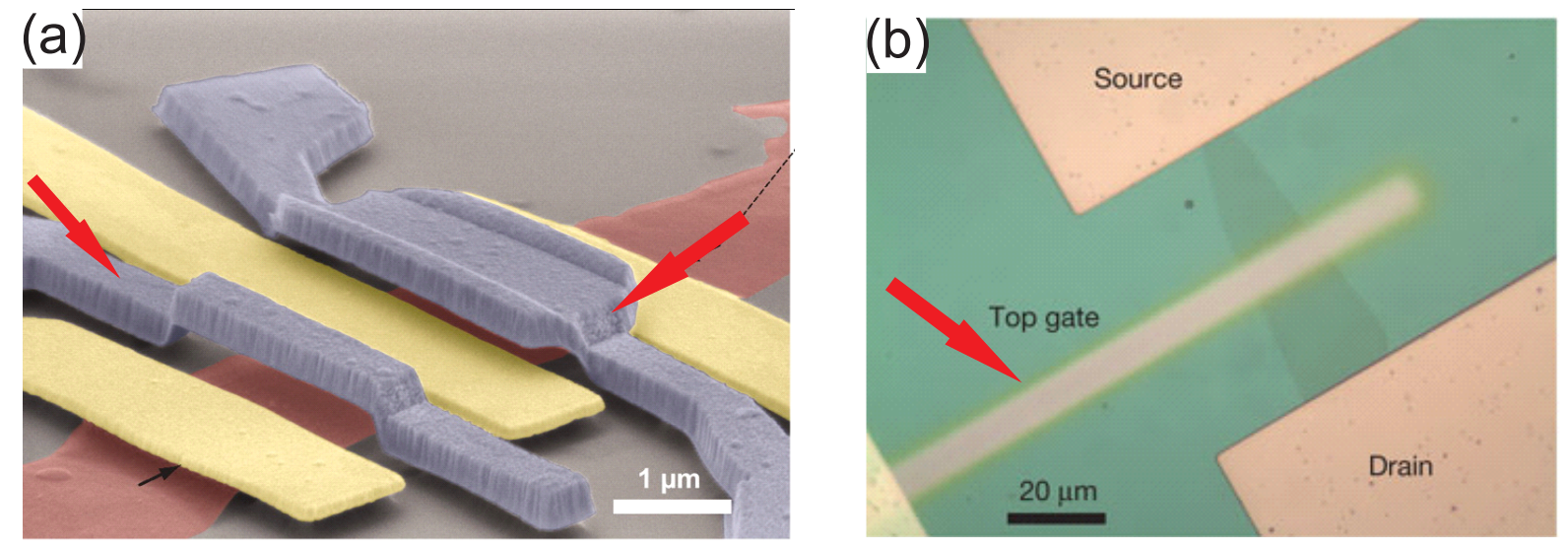

Figura 4.4: (a)Micrografia eletrônica de varredura, colorida artificialmente, de uma bicamada de grafeno suspensa entre eletrodos de uma liga de $\mathrm{Cr} / \mathrm{Au}$ e na presença de um top gate. Imagem retirada da referência [69]. (b)Imagem de microscopia ótica de uma bicamada de grafeno suportada sobre um substrato de $\mathrm{SiO}_{2}$, entre dois eletrodos de ouro e na presença de um eletrodo de top gate. Imagem retirada da referência [7]

A proposta de trabalho apresentado neste capítulo da tese foi motivada no entendimento das propriedades intrínsecas de nanodispositivos baseados em bicamadas de grafeno, sem o efeito de agentes externos como interações com o substrato e dopagens externas. Experimentalmente a proposta assemelha-se muito com a imagem apresentada na figura 4.4(a), onde a bicamada de grafeno está suspensa no dispositivo. 


\subsubsection{Procedimento de cálculo e setup utilizado}

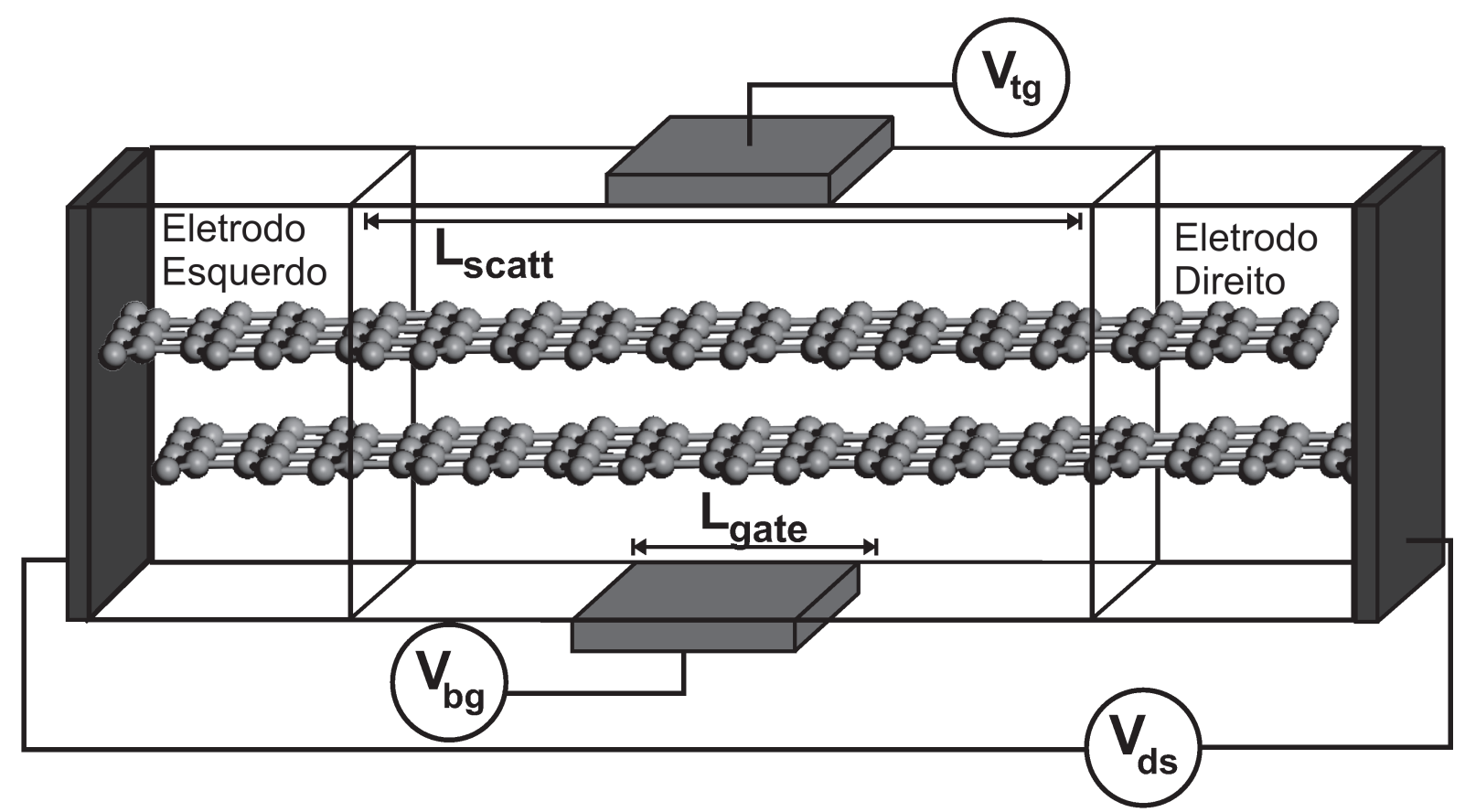

Figura 4.5: Visão esquemática do nanotransistor de bicamada de grafeno com duplo gate que consideramos em nossas simulações. $V_{d s}, V_{b g}, V_{t g}, L_{s c a t t}, L_{g a t e}$ correspondem a voltagem fonte-dreno, voltagem de back-gate, voltagem de top-gate, comprimento da região de espalhamento e comprimento do gate, respectivamente.

Na Figura 4.5, mostramos uma representação esquemática do nanotransistor de bicamada de grafeno com duplo gate, que foi utilizado em nossos cálculos. A geometria atômica é composta de uma bicamada de grafeno, com empilhamento do tipo AB (Bernal), com uma região de espalhamento de aproximadamente $15 \mathrm{~nm}$, conectada aos eletrodos esquerdo e direito. Uma voltagem fonte-dreno $\left(V_{d s}\right)$, é aplicada através do sistema, definindo a direção da corrente. As propriedades do transporte de carga foram calculadas com o formalismo de NEGF-DFT descrito na seção 3.2, que usa as Hamiltonianas de Kohn-Sham e matrizes de overlap calculadas com o código SIESTA. A matriz densidade do sistema é obtida de maneira auto-consistente. Para investigar um sistema $2 D$, com um duplo gate finito, incluímos nas simulações: i) Pontos $k$ transversais à direção de transporte $\left(k_{\perp}\right)$, onde para cada ponto $k_{\perp}$ no espaço recíproco, obtemos uma densidade $k_{\perp}$-dependente, $D\left(k_{\perp}\right)_{\mu \nu}$, por meio da integração da função de Green Lesser, e a transmitância, $T\left(E, k_{\perp}\right)$, dada por

$$
T\left(E, k_{\perp}\right)=\operatorname{Tr}\left[\Gamma_{L}^{k_{\perp}}(E) G^{a}\left(E, k_{\perp}\right) \Gamma_{R}^{k_{\perp}}(E) G^{r}\left(E, k_{\perp}\right)\right]
$$


$G^{r(a)}\left(E, k_{\perp}\right)$ é a função de Green retardada(avançada), enquanto que, $\Gamma_{L(R)}^{k_{\perp}}(E)$ são os termos que representam o acoplamento entre os eletrodos e a região de espalhamento. A densidade total e a transmitância são obtidas através da integração no espaço recíproco, a partir das seguintes equações:

$$
\begin{aligned}
D_{\mu \nu} & =\frac{1}{(2 \pi)^{2}} \int d k_{\perp}^{2} D\left(k_{\perp}\right)_{\mu \nu}, \\
T(E) & =\frac{1}{(2 \pi)^{2}} \int d k_{\perp}^{2} T\left(E, k_{\perp}\right) .
\end{aligned}
$$

Com a inclusão de pontos $k$ podemos realizar a simulação de sistemas $2 D$. Uma vez a matriz densidade esteja convergida, a corrente é calculada utilizando o modelo de LandauerBüttiker [62] na abordagem não interagente de Meir-Wingreen [58], onde a corrente é dada por:

$$
I=\frac{2 e}{h} \int_{-\infty}^{\infty} T(E)\left[f\left(E-\mu_{L}-f\left(E-\mu_{R}\right)\right)\right] d E
$$

$T(E)$ é obtida a partir da equação (4.4) e $f\left(E-\mu_{L / R}\right)$ são as funções de distribuição de FermiDirac para os eletrodos esquerdo(L) e direito(R). Para os cálculos utilizamos a aproximação local da densidade (LDA) para o funcional de troca e correlação [66], pois ele descreve corretamente as interações entre as camadas do grafeno sem a inclusão de correções de van der Waals [70]; ii) Incluímos também um método de Multigrid para a solução da equação de Poisson no espaço real, que permite soluções não periódicas para o potencial de Hartree, necessárias para a utilização de diferentes valores para os potenciais de top gate $\left(V_{t g}\right)$ e back gate $\left(V_{b g}\right)$ com comprimentos de gate finitos. Assim, resolvemos a equação de Poisson em uma caixa retangular fixando as condições de contorno superiores e inferiores. Os valores de $V_{b g}$ e $V_{t g}$ são definidos somente em uma região finita de comprimento $L_{\text {gate }}($ Veja figura 4.5). Em nossas investigações consideramos $L_{\text {gate }}=1,2,3,4,5$ e $10 \mathrm{~nm} ; L_{\text {scatt }} \approx 15 \mathrm{~nm}$; e a distância entre os contornos superior e inferior é de $2 \mathrm{~nm}$.

Na figura 4.6, mostramos uma imagem de uma seção longitudinal da diferença entre o potencial de Hartree do sistema na presença de um gate com o potencial do sistema sem campo. Como podemos observar, o campo é aplicado em somente uma região finita do sistema.

A convergência do sistema é garantida utilizando-se uma base dupla- $\zeta$ e $300 R y$ para o mesh cut-off. A transmitância é calculada com 1000 pontos $k$ na zona de Brillouin com o esquema de Monkhorst-Pack [71]. Utilizamos uma geometria completamente relaxada com uma distância carbono-carbono de $1.428 \AA$ e uma distância entre camadas de $3.203 \AA$. 


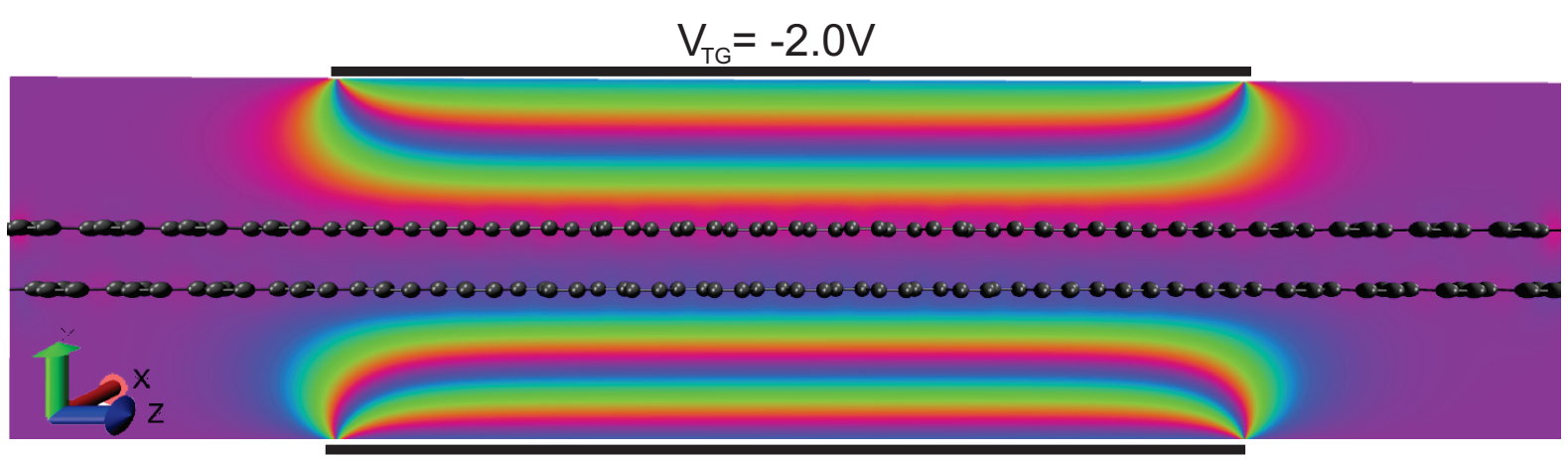

$$
\mathrm{V}_{\mathrm{BG}}=+2.5 \mathrm{~V}
$$

Figura 4.6: Seção longitudinal da região do sistema na presença dos potenciais de back/top gate. A imagem mostra a diferença entre o potencial eletrostático de um sistema na presença de campo e o sistema sem campo.

\subsubsection{Resultados}
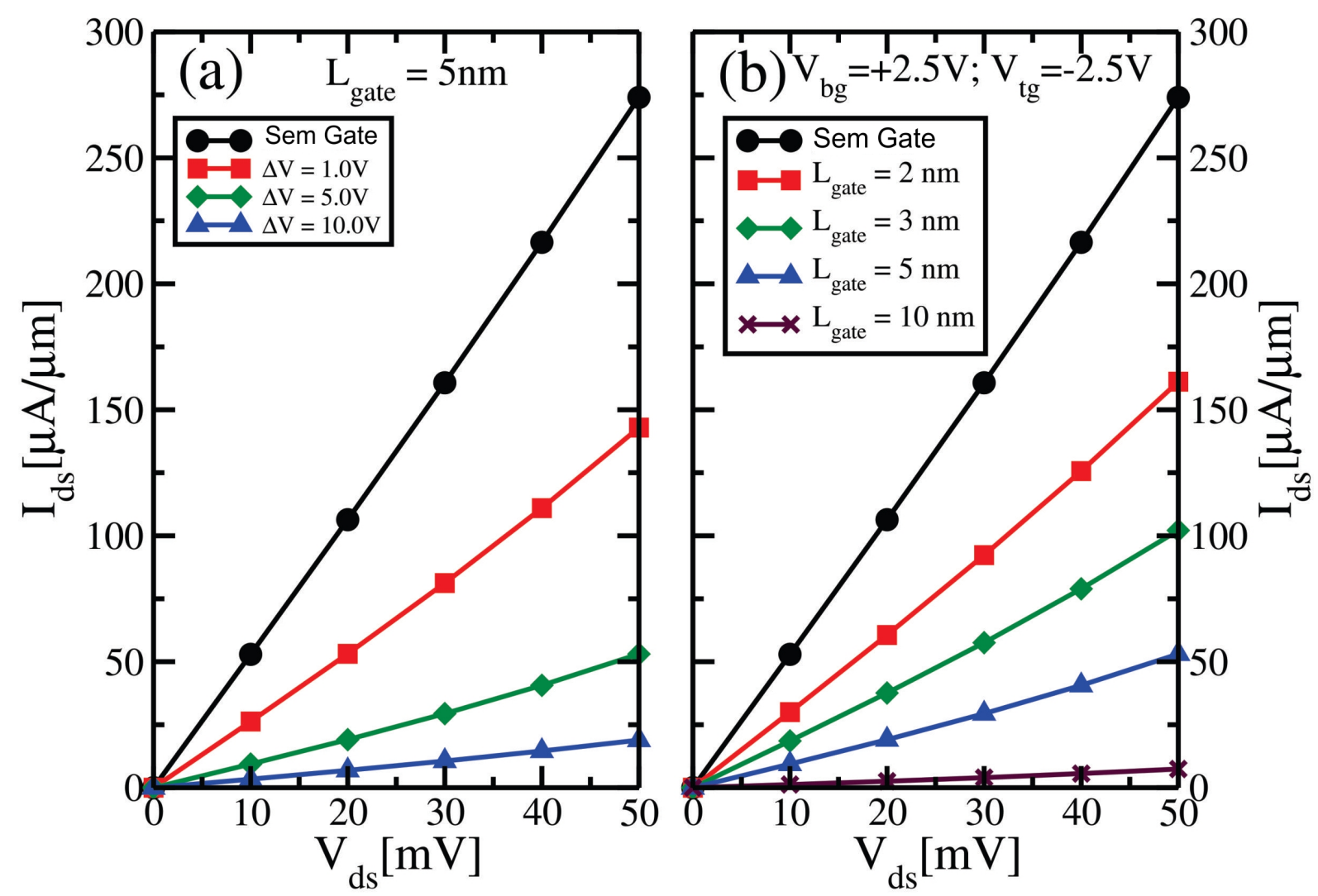

Figura 4.7: Em (a) apresentamos as curvas $I_{d s} \times V_{d s}$ variando $\Delta V$, para $300 K$ e $L_{\text {gate }}=5 \mathrm{~nm}$. Em (b) apresentamos as curvas $I_{d s} \times V_{d s}$ variando $L_{\text {gate }}$ para $300 \mathrm{~K}$ e $\Delta V=5.0 \mathrm{~V}$

Na Figura 4.7, mostramos a corrente $I_{d s}$ em função da voltagem fonte-dreno $V_{d s}$ para temperatura ambiente. Em nossas simulações as voltagens de gate $V_{b g}$ e $V_{t g}$ são sintonizadas independentemente. Na figura 4.7, todas as curvas são para voltagens de gate com $V_{b g}=-V_{t g}$, onde 
a diferença de voltagem $\Delta V=2\left|V_{b g}\right|$. Na figura 4.7(a), usamos $\Delta V=1.0 \mathrm{~V}, 5.0 \mathrm{~V}$ e $10.0 \mathrm{~V}$, gerando campos elétricos de 0.5, 2.5 e $5.0 \mathrm{~V} / \mathrm{nm}$, comparável com os valores experimentais. Em todos os experimentos $[7,72,73]$, o deslocamento do campo elétrico na bicamada é estimado através da constante dielétrica e espessura da camada do substrato dielétrico utilizado, e também a partir do potencial efetivo devido à dopagens provenientes das condições experimentais. O campo elétrico foi calculado via relação: $\Delta V / d_{b t}$, onde $d_{b t}=2 n m$ é a distância entre o gate superior e inferior. Para as configurações estudadas, verificamos um decréscimo da corrente quando $\Delta V$ aumenta, sugerindo a abertura de um gap de energia que aumenta com $\Delta V$. Além disso, para um dado comprimento e uma dada voltagem de gate, a corrente elétrica possui uma dependência linear com a voltagem fonte-dreno, indicando um contato ôhmico entre as regiões com o gate e sem o gate. Este comportamento também é observado experimentalmente $[59,60,72]$. Na figura 4.7(a), mostramos que a corrente elétrica nunca vai para zero, mesmo para $\Delta V=10.0 \mathrm{~V}$, onde este resultado está em concordância com trabalhos experimentais que fabricam dispositivos com bicamadas de grafeno e duplos gates.

$\mathrm{Na}$ figura 4.7(b), nós fixamos $V_{b g}=+2.5 \mathrm{~V}$ e $V_{t g}=-2.5 \mathrm{~V}$, e apresentamos a dependência da corrente com o comprimento do gate, $L_{\text {gate }}$, com $L_{\text {gate }}=2,3,4,5$ e $10 \mathrm{~nm}$. Observamos, novamente, um decréscimo na corrente com o aumento do $L_{\text {gate, }}$ indicando um aumento da região com gap. Novamente, o sistema nunca atinge uma corrente zero, mesmo com $L_{\text {gate }}=$ $10 \mathrm{~nm}$.

Como utilizamos condições de contorno aberta, com o potencial químico do sistema fixado pelos dois eletrodos, podemos variar independentemente $V_{b g}$ e $V_{t g}$ para controlar o tipo dos portadores de carga na região de espalhamento. A presença de um campo elétrico externo no sistema leva uma quebra de simetria entre as duas camadas. Como resultado, existe a abertura de um gap de energia e uma transferência de carga entre as camadas. Se não houvesse nenhuma transferência de carga, a condição $V_{b g}=-V_{t g}$ iria definir o ponto de neutralidade de carga do sistema (CNP). Entretanto, a presença de um termo de correção de Hubbard, mesmo no nível de campo médio, acoplado com esta transferência de carga, leva a um deslocamento das bandas na região do gate. Desta forma, o CNP ocorre para $V_{b g} \neq V_{t g}$, como mostrado na figura 4.8.

Assim, realizamos cálculos, onde fixamos $V_{b g}$ em um certo valor $(+2.5 \mathrm{~V})$ e variamos $V_{t g}$, como é feito experimentalmente [72]. As correntes fonte-dreno calculadas e a densidade de portadores na bicamada, em função da voltagem de top-gate, $V_{t g}$, são mostradas na figura 4.8 

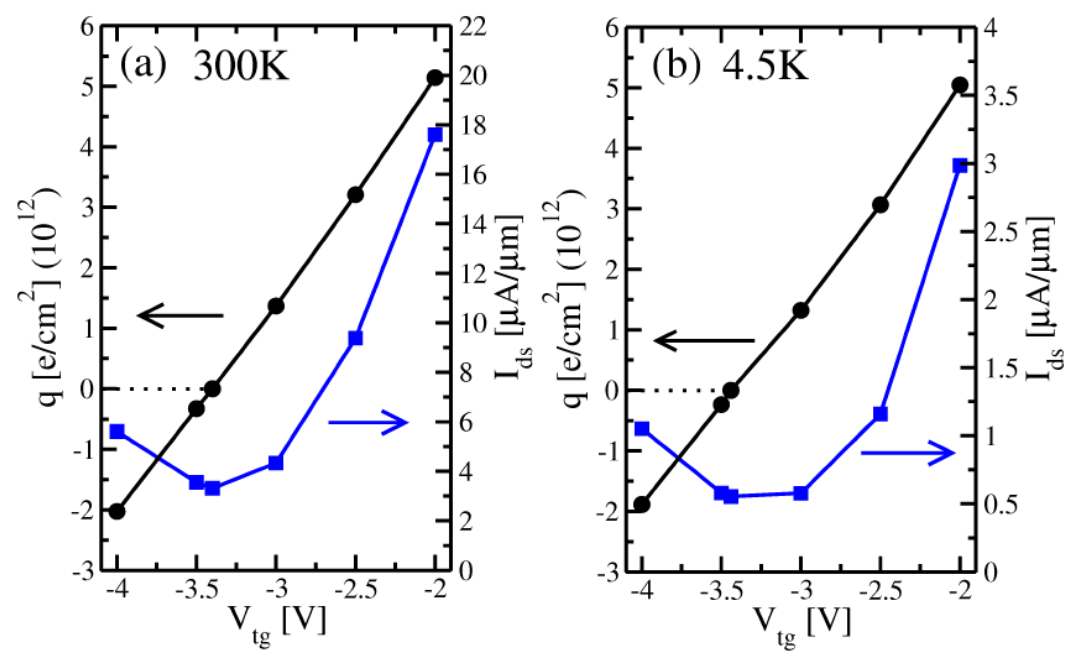

Figura 4.8: Características do transporte eletrônico de um FET de bicamada de grafeno com duplo gate, para $L_{\text {gate }}=5 \mathrm{~nm}$ e $V_{b g}=+2.5 \mathrm{~V}$. A corrente (quadrados azuis) e carga induzida (círculos pretos) à (a) Temperatura ambiente e (b) $\mathrm{T}=4.5 \mathrm{~K}$.

para um comprimento de gate de $L_{\text {gate }}=5 \mathrm{~nm}$ e $T=300 \mathrm{~K}(\mathrm{a})$ e $T=4.5 \mathrm{~K}(\mathrm{~b})$, respectivamente. A voltagem fonte-dreno foi fixada em $10 \mathrm{mV}$. O mínimo de corrente em ambas as curvas corresponde ao ponto de neutralidade de carga. Para $V_{t g}>3.4 V$, elétrons são injetados no sistema, enquanto que para $V_{t g}<3.4 \mathrm{~V}$, buracos são injetados. Como não temos nenhuma dopagem externa, no CNP, o potencial químico do sistema está no meio do gap. Além disso, como as bandas de valência e condução são muito similares, a temperatura irá criar portadores intrínsecos, mas não irá modificar a posição do potencial químico. Conseqüentemente, existirá alguma transferência de carga dos eletrodos (elétrons ou buracos), dependendo do shift das bandas na região do gate, com relação ao potencial químico dos eletrodos. Na figura 4.8 também plotamos a concentração dos portadores de carga por unidade de área, onde este apresenta um comportamento linear em função da variação da voltagem $V_{t g}$, com carga zero no ponto de neutralidade de carga. Isso indica um deslocamento linear das bandas com a voltagem de gate.

É notável um decréscimo da corrente quando a temperatura diminui de $300 \mathrm{~K}$ para $4.5 \mathrm{~K}$. Isso é causado por pequenas variações na transmitância, mas principalmente por mudanças nas funções de distribuição de Fermi-Dirac. Para o caso apresentado na figura 4.8, a corrente diminui mais de 6 vezes. Entretanto, a concentração de portadores é muito similar para ambas temperaturas, como resultado da invariância da posição do meio do gap com a temperatura, como discutido anteriormente.

A figura 4.9, mostra o comportamento da corrente fonte-dreno para diferentes comprimen- 

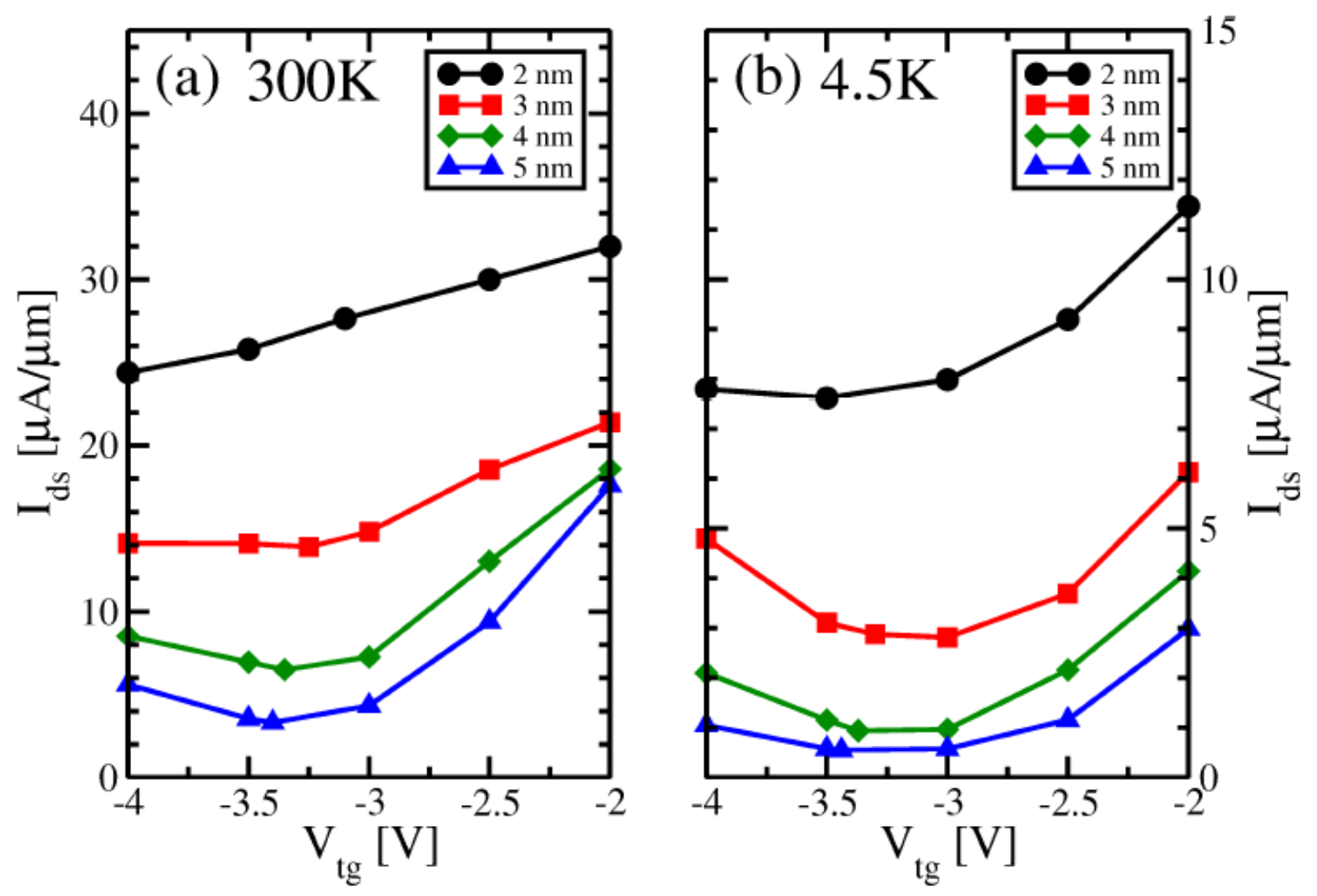

Figura 4.9: $I_{d s}$ em função de $V_{t g}$ variando-se $L_{g a t e}$ à temperatura (a) $300 \mathrm{~K}$ e (b) $4.5 \mathrm{~K}$. A voltagem fonte-dreno foi mantida em $10 \mathrm{mV}$ e $V_{b g}=+2.5 \mathrm{~V}$.

tos de gate como função da voltagem de top-gate. Novamente, fixamos $V_{b g}=+2.5 \mathrm{~V}$ e realizamos os cálculos para temperaturas de $300 \mathrm{~K}$ e $4.5 \mathrm{~K}$. Para $L_{\text {gate }}=2 \mathrm{~nm}$, em contraste com os outros comprimentos de gate, não observamos um mínimo na corrente no ponto de neutralidade de carga para ambas as temperaturas. Se aumentarmos o comprimento do gate, observamos um mínimo de condutância no ponto de neutralidade de carga, como esperávamos.

Para entender porque não existe um mínimo de corrente no ponto de neutralidade de carga para gates pequenos, $<2 \mathrm{~nm}$, temos que entender os principais pontos que contribuem para a corrente neste sistema.

O valor da corrente para cada valor de top-gate depende principalmente de dois pontos, para uma determinada temperatura: (i) posicionamento da transmitância em relação ao nível de Fermi dos eletrodos. Este posicionamento está fortemente ligado com a quantidade de carga que é induzida pelo gate, ou seja, quanto que a transmitância do sistema se desloca para cada valor de top-gate ${ }^{2}$. Isso é muito importante, pois a função de distribuição de Fermi-Dirac está

\footnotetext{
${ }^{2}$ No ponto de neutralidade de carga, o sistema está alinhado com o nível de Fermi dos eletrodos
} 

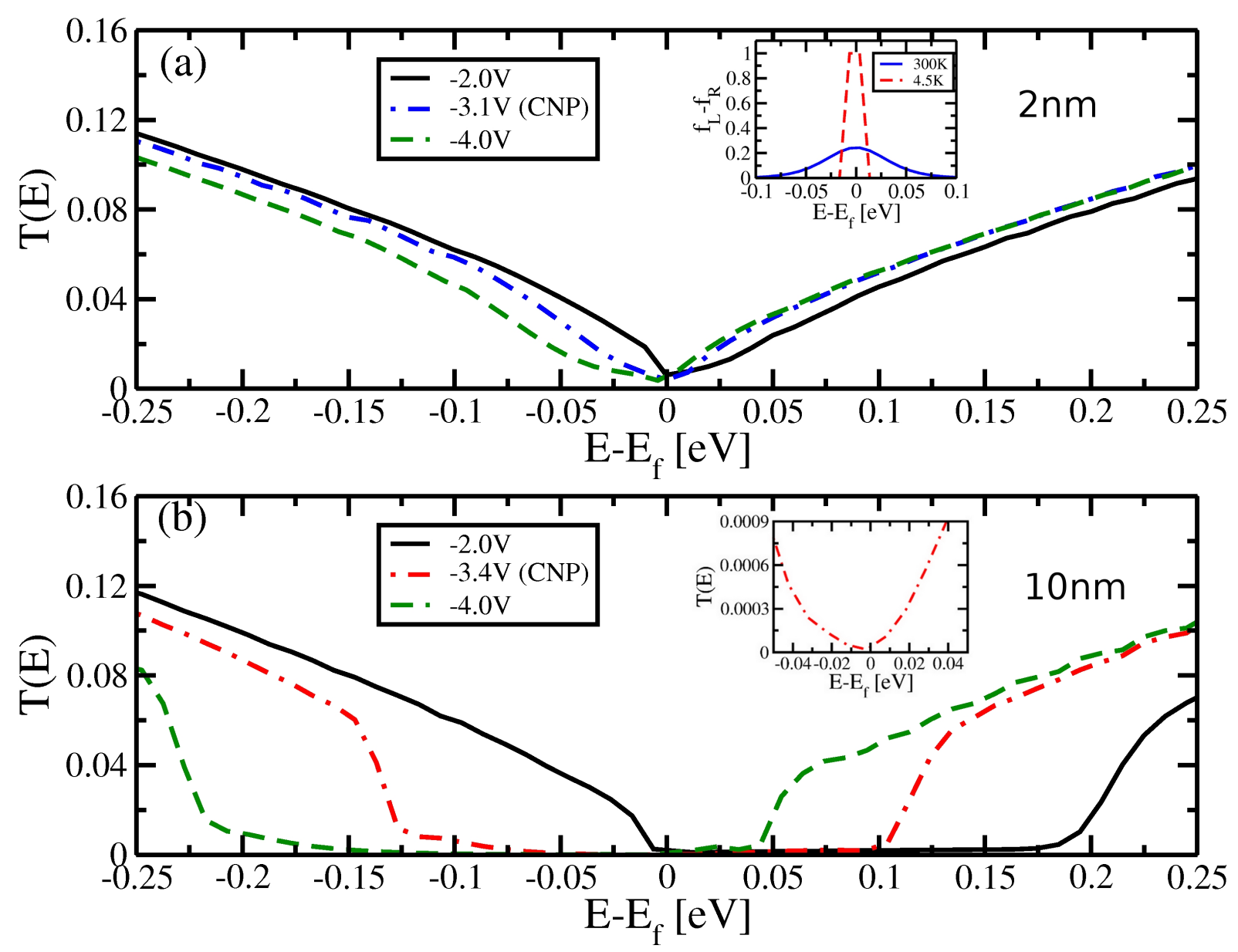

Figura 4.10: (a) Transmitâncias para $L_{\text {gate }}=2 \mathrm{~nm}$, variando-se $V_{t g}$. Inset(a) mostra a diferença das funções de Fermi-Dirac dos eletrodos para $4.5 \mathrm{~K}$ e $300 \mathrm{~K}$ com uma tensão de bias de $10 \mathrm{mV}$. (b) Transmitância para $L_{\text {gate }}=10 \mathrm{~nm}$, variando-se $V_{t g}$. Inset(b) Detalhe da transmitância para $L_{\text {gate }}=10 \mathrm{~nm}$ no ponto de neutralidade de carga, em uma região ao redor do nível de Fermi.

centralizado com relação ao nível de Fermi dos eletrodos (Observar inset na figura 4.10(a)); (ii) Valor da transmitância no intervalo de energia definido pela função de Fermi-Dirac. Este valor está fortemente correlacionado com o comprimento do gate e a intensidade do campo.

Desse modo, existe uma competição no valor da corrente seguindo os dois itens anteriores. Para um gate pequeno, da ordem de $2 \mathrm{~nm}$, a diminuição na transmitância devido à variação do campo elétrico é sempre maior que o shift adquirido pelo sistema, devido à variação do valor do top-gate. Este comportamento pode ser observado na figura 4.10(a) para o caso do gate de $2 n m$. Sendo assim, como a transmitância na região de importância para o cálculo da corrente (Definida pela função de Fermi-Dirac), está diminuindo mais do que se deslocando, a corrente sempre cai nesse intervalo de voltagem, como observado na figura 4.9. Entretanto, quando vamos para um sistema de $10 \mathrm{~nm}$, o posicionamento da transmitância em relação ao nível de 
Fermi do eletrodos é o mais importante, pois se observamos a figura 4.10(b), a região de baixa transmitância modifica muito pouco com a variação da voltagem de top-gate, mas desloca-se consideravelmente.

A figura 4.9, mostra o efeito do potencial de gate para (a) $L_{\text {gate }}=2 \mathrm{~nm}$ e (b) $L_{\text {gate }}=$ $10 \mathrm{~nm}$ à $300 \mathrm{~K}$ com $V_{b g}+2.5 \mathrm{~V}$, variando-se $V_{t g}$. Na presença das mesmas condições, para gates grandes as modificações na transmitância são mais expressivas em comparação com os menores. Para cada configuração de $V_{b g} / V_{t g}$, os potenciais de gate geram uma região de baixa transmitância que é mais pronunciada com $L_{\text {gate }}=10 \mathrm{~nm}$ em comparação com $L_{\text {gate }}=2 \mathrm{~nm}$. Entretanto, a transmitância nunca vai para zero, como pode ser visto no inset na figura 4.9(b).

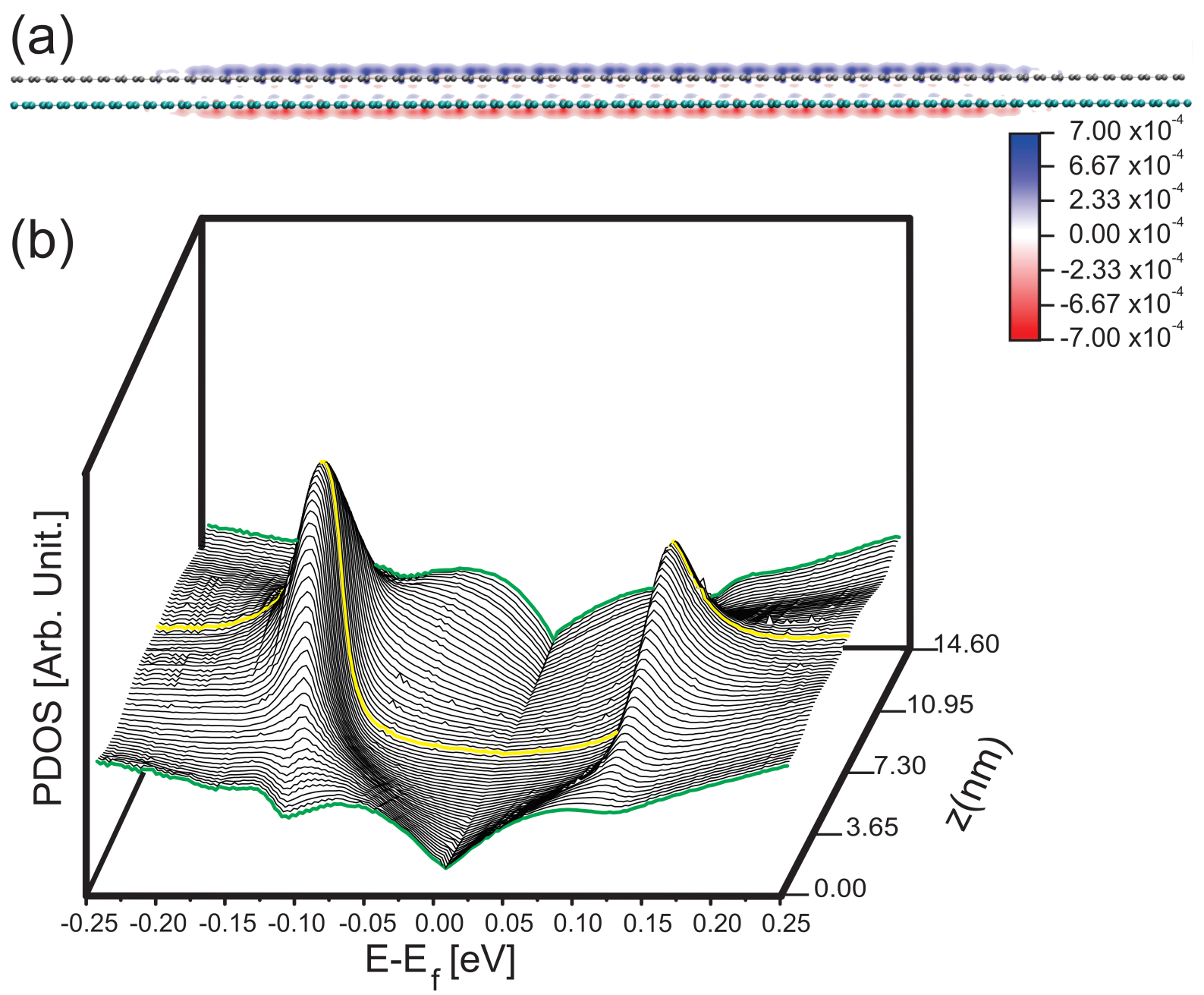

Figura 4.11: (a) Diferença local entre as cargas de um sistema sem gate com um sistema na presença de um gate. Como pode ser visto, existe uma transferência de elétrons a partir da camada superior para a inferior(Unidades em $e / b o h r^{3}$ ). (b) Densidade de estados projetada a cada quatro átomos (equivalentes à célula unitária da $\mathrm{BG}$ ) ao longo da direção $z$. O sistema considerado aqui possui $L_{\text {gate }}=10 \mathrm{~nm}$ e está no ponto de neutralidade de carga. 
É importante enfatizar que em nossos cálculos o campo elétrico é aplicado somente em uma região finita da região de espalhamento através do sistema. Como consequiência, a transferência de carga entre as camadas causada pelo campo elétrico só ocorre na região dos gates. Na figura 4.11(a), mostramos a carga induzida no ponto de neutralidade de carga, por um sistema com um gate de $L_{\text {gate }}=10 \mathrm{~nm}$. Na região inferior(superior) das camadas existe um excesso(falta) de elétrons em vermelho(azul). Na figura 4.11(b), mostramos a densidade de estados projetada a cada quatro átomos (equivalentes à célula unitária da bicamada de grafeno) ao longo de todo o comprimento da região de espalhamento, desde o eletrodo esquerdo $(z=0.0 \mathrm{~nm})$ até o direito $(z=14.6 \mathrm{~nm})$. Perto da região dos eletrodos (linhas verdes) a densidade de estados projetada (PDOS) são similares à bicamada de grafeno, sem gate, como esperado, enquanto que em uma região logo abaixo do gate (linha amarela) a PDOS é característica de uma bicamada de grafeno com um campo elétrico aplicado perpendicularmente às camadas, onde os dois picos localizados em $\pm 12 m e V$ são associados com a presença do comportamento tipo chapéu mexicano das bandas. Entretanto, desde que o gate é espacialmente finito, mesmo com a presença de um gap de energia nas bandas, existe sempre a presença de uma densidade de estados finita. Isso é devido à penetração da função de onda na região de energia proibida. Esse resultado explica a ausência de um gap de energia para o transporte.

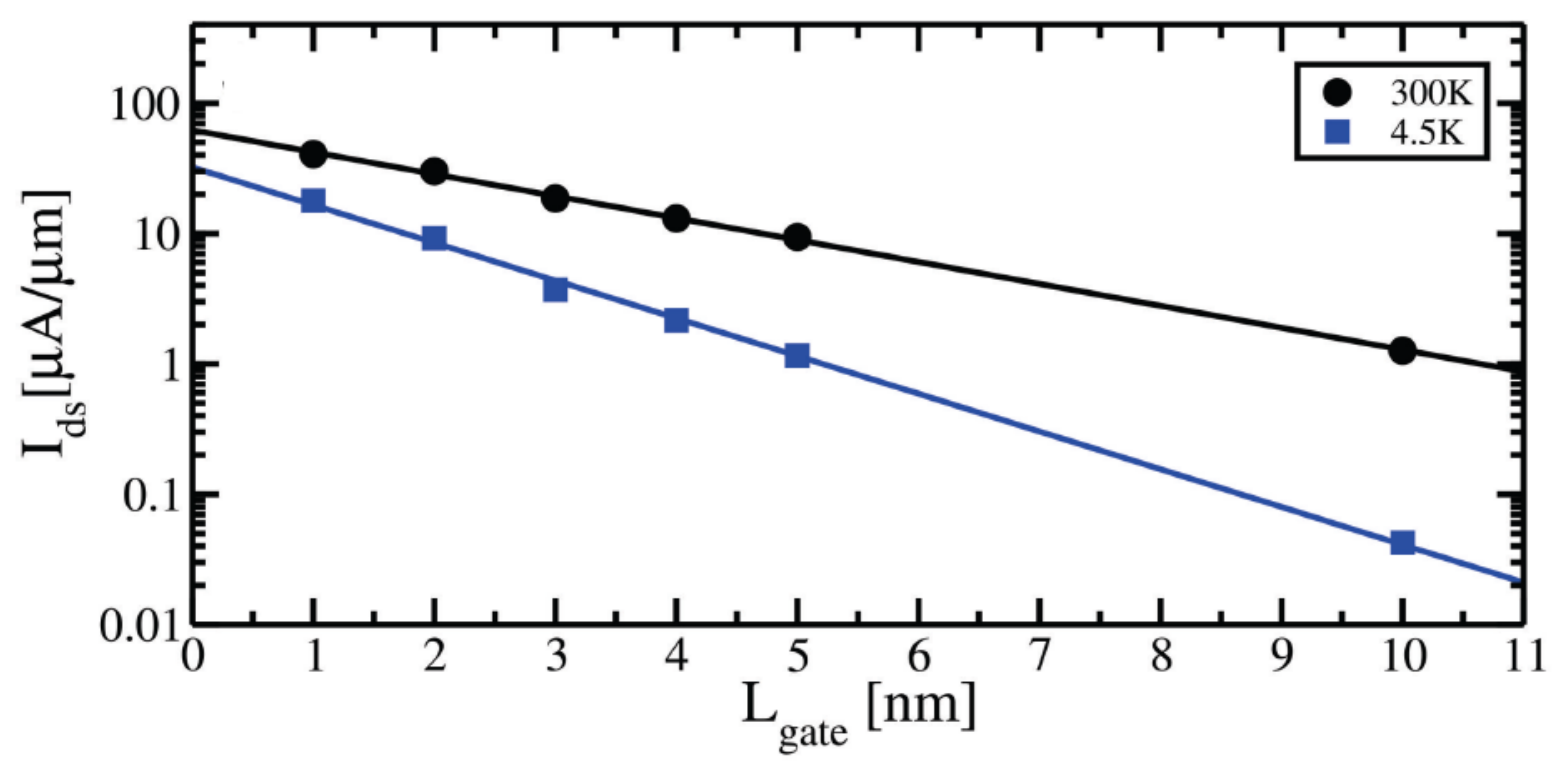

Figura 4.12: Curvas $I_{d s} \times L_{\text {gate }}$ para $\Delta V=5.0 \mathrm{~V}$ e $V_{d s}=10 \mathrm{mV}$, para $300 \mathrm{~K}$ e $4.5 \mathrm{~K}$. Para todos os gráficos, $\Delta V=2\left|V_{t g}\right|$, com $V_{b g}=-V_{t g}$.

Além do mais, para entender porque a corrente que sempre existe no sistema, olhamos para 
o seu comportamento em função do comprimento do gate. Na figura 4.12, fixamos $\Delta V=5 \mathrm{~V}$ e $V_{d s}=10 \mathrm{mV}$, e variamos $L_{\text {gate }}$ para $300 \mathrm{~K}$ e $4.5 \mathrm{~K}$. Obtemos uma dependência exponencial entre a corrente e o comprimento do gate, característico de um regime de tunelamento. A corrente de tunelamento persiste mesmo para o maior gate que consideramos, $L_{\text {gate }}=10 \mathrm{~nm}$. Um aumento significante na corrente, por uma ou duas ordens de grandeza, com a temperatura pode ser observado; isso é em grande parte causado pelo alargamento da janela de transporte através de $\left(f\left(E-\mu_{L}\right)-f\left(E-\mu_{R}\right)\right)$. Em um regime de bias pequeno, a cauda de função de Fermi-Dirac é o que controla a corrente, desde existe uma pequena densidade de estados (e conseqüentemente um pequeno número de canais de transporte) ao redor do nível de Fermi dos eletrodos. É importante lembrar que nos cálculos de primeiros princípios mantemos todas as características intrínsecas do dispositivo, sem a presença de defeitos, impurezas e dopagens externas, ou materiais dielétricos como substrato. Conseqüentemente, a corrente off nas simulações são explicadas por um tunelamento através da região do gate e depende fortemente da janela de transporte causada pela distribuição de Fermi-Dirac para uma dada temperatura.

Estimamos para o nanodispositivo, a razão on/off das correntes, $\left(I_{o n} / I_{o f f}\right)$ à temperatura ambiente $(300 K)$, e à baixa temperatura $(4.5 K)$. A corrente on, é calculada para a bicamada sem a presença do gate com uma voltagem fonte dreno de $10 \mathrm{mV}$, e as correntes off são todas calculadas no ponto de neutralidade de carga, com uma voltagem de back gate de $+2.5 \mathrm{~V}$ com uma voltagem $V_{d s}=10 \mathrm{mV}$. A maior $I_{o n} / I_{o f f}$ ocorre para $L_{g a t e}=10 \mathrm{~nm}$, que é por volta de 100 à temperatura ambiente, e 1250 à $4.5 K$.

\subsection{Conclusões}

Em resumo, usando cálculos de primeiros princípios, estudamos as propriedades de transporte de um nanotransistor de bicamada de grafeno com duplo gate. Mostramos que sobre a ação de um campo elétrico externo, mesmo para comprimentos de gate de $100 \AA$ a temperatura ambiente e a $4.5 K$, uma corrente não nula sempre é exibida. Os resultados podem ser explicados pela presença de um regime de tunelamento devido a estados remanescentes na região do gap. Obtemos também razões on/off para a corrente de 100 à $300 \mathrm{~K}$ e 1250 à 4.5K, que são valores próximos aos obtidos experimentalmente [72]. 


\section{Capítulo 5}

\section{Propriedades Eletrônicas e de Transporte de Multicamadas de Grafeno}

Quando duas ou mais monocamadas de grafeno são empilhadas, elas formam as chamadas multicamadas de grafeno(FLG) ${ }^{1}$. Um exemplo deste tipo de sistema, as bicamadas de grafeno apresentam um gap de energia na presença de um campo elétrico externo, que pode ser sintonizado através da aplicação de um do campo [7,74,75]. ${ }^{2}$.

Quando mais de duas camadas de grafeno são empilhadas ocorre o surgimento de mais um grau de liberdade no sistema: a ordem do empilhamento entre as camadas. Este novo grau de liberdade exerce uma forte influência na estrutura eletrônica das FLG. [34, 76-79]. Dois tipos de empilhamentos já foram verificados experimentalmente: (i)(AB) ou Bernal, que é um empilhamento com simetria hexagonal; (ii)(ABC) ou romboédrico, que é um empilhamento com simetria romboédrica. Este último empilhamento existe tanto no grafite natural quanto no sintetizado em laboratório, em uma certa quantidade além da fração desordenada [80]. Dessa maneira, é possível a obtenção de sistemas de multicamadas de grafeno, com ambos os empilhamento, ou seja, podemos ter tricamadas ABA e ABC, tetracamadas ABAB e ABCA, pentacamadas ABABA e ABCAB, etc.

Foram realizados alguns trabalhos teóricos recentes utilizando uma abordagem tight-biding, para investigar o efeito do número de camadas e do campo elétrico em filmes de grafeno com empilhamentos do tipo Bernal e romboédrico [81]. Koshino [34] comparou as estruturas eletrônicas tight-binding de empilhamentos $\mathrm{AB}$ e $\mathrm{ABC}$ na presença de campos elétricos e encontrou que nas estruturas $\mathrm{ABC}$ um gap de energia é aberto, enquanto que nas do tipo $\mathrm{AB}$

\footnotetext{
${ }^{1}$ Do inglês Few Layer Graphene.

${ }^{2}$ Para uma descrição mais detalhada das bicamadas de grafeno veja capítulo 4
} 
com mais de duas camadas, o sistema permanece semimetálico. Estruturas de bandas de ambos empilhamentos $(A B)$ e ( $A B C)$ em sistemas de algumas camadas foram também estudados com a teoria do funcional da densidade (DFT) - sem campo elétrico externo por Latil e Henrard [76] e com campo elétrico externo por Aoki e Amawashi [77]. A resposta ao campo elétrico na estrutura de bandas de uma tricamada $\mathrm{ABC}$ foi investigada por Zhang et al. [82]. No lado experimental as tricamadas $\mathrm{ABA}$ e $\mathrm{ABC}$, tetracamadas $\mathrm{ABAB}$ e $\mathrm{ABCA}$ e pentacamadas $\mathrm{ABABA}$ e $\mathrm{ABCAB}$, já foram isoladas e recentemente observadas por espectroscopia de absorção de infravermelho [83-87].

Neste capítulo, iremos estudar as propriedades eletrônicas e de transporte das multicamadas de grafeno. Através de cálculos baseados na teoria do funcional da densidade, determinaremos qual a influência dos possíveis empilhamentos na estrutura eletrônica desses sistemas, na presença e ausência de campos elétricos externos. Estudaremos também, através de cálculos de primeiros princípios baseados no formalismo de funções de Green fora do equilíbrio combinado com a teoria do funcional da densidade, a influência do empilhamento nas propriedades de transporte desses sistemas. Mostraremos que o transporte é altamente sensíveis a esse novo grau de liberdade de empilhamento. Para as propriedades de transporte iremos considerar sistemas de 2 até 12 camadas, em ambos os empilhamentos, onde mostraremos que no empilhamento romboédrico ${ }^{3}$, uma simples contagem de bandas não fornece toda a informação das propriedades de transporte desses sistemas. Mostraremos também que o transporte no empilhamento romboédrico é dominado pelas camadas mais externas do sistema, enquanto no Bernal, todo o sistema contribui para o transporte. Além disso, mostraremos que é possível diferenciar o tipo de empilhamento do sistema, através da temperatura e voltagem de bias.

\subsection{Energética dos possíveis empilhamentos das FLG}

O grafite do tipo Bernal ( $\alpha$-grafite) é mais abundante na natureza que a sua fase romboédrica - ( $\beta$-grafite) $[88,89]$ A diferença estrutural mais importante entre os empilhamentos $\mathrm{AB}\left(\alpha\right.$-grafite) e $\mathrm{ABC}$ ( $\beta$-grafite), é que o $\mathrm{AB}$ apresenta uma linha de ligações do tipo $p_{z}$, conectando todos os átomos de uma única subrede do grafeno, perpendicularmente às camadas através de todo o sistema. No empilhamento $(\mathrm{ABC})$, apenas pares de átomos individuais são

\footnotetext{
${ }^{3}$ Todos os sistemas considerados são pristinos
} 
conectados perpendicularmente às camadas. Na figura 5.1 mostramos esquematicamente como se dão esses tipos de empilhamentos nas tricamadas, tetracamadas e pentacamadas de grafeno. Em outras palavras, nos empilhamentos $(\mathrm{AB})$ existem dois tipos de átomos, um com ligações $p_{z}$ em ambas as direções $z$, e outro sem ligações $p_{z}$, enquanto que nos sistemas (ABC) cada átomo tem um vínculo $p_{z}$, seja em $z$ positivo ou negativo. Evidentemente, esta diferença deve ter um grande impacto sobre a estrutura das bandas, mesmo com a interação entre as camadas sendo muito fraca, do tipo van der Waals.
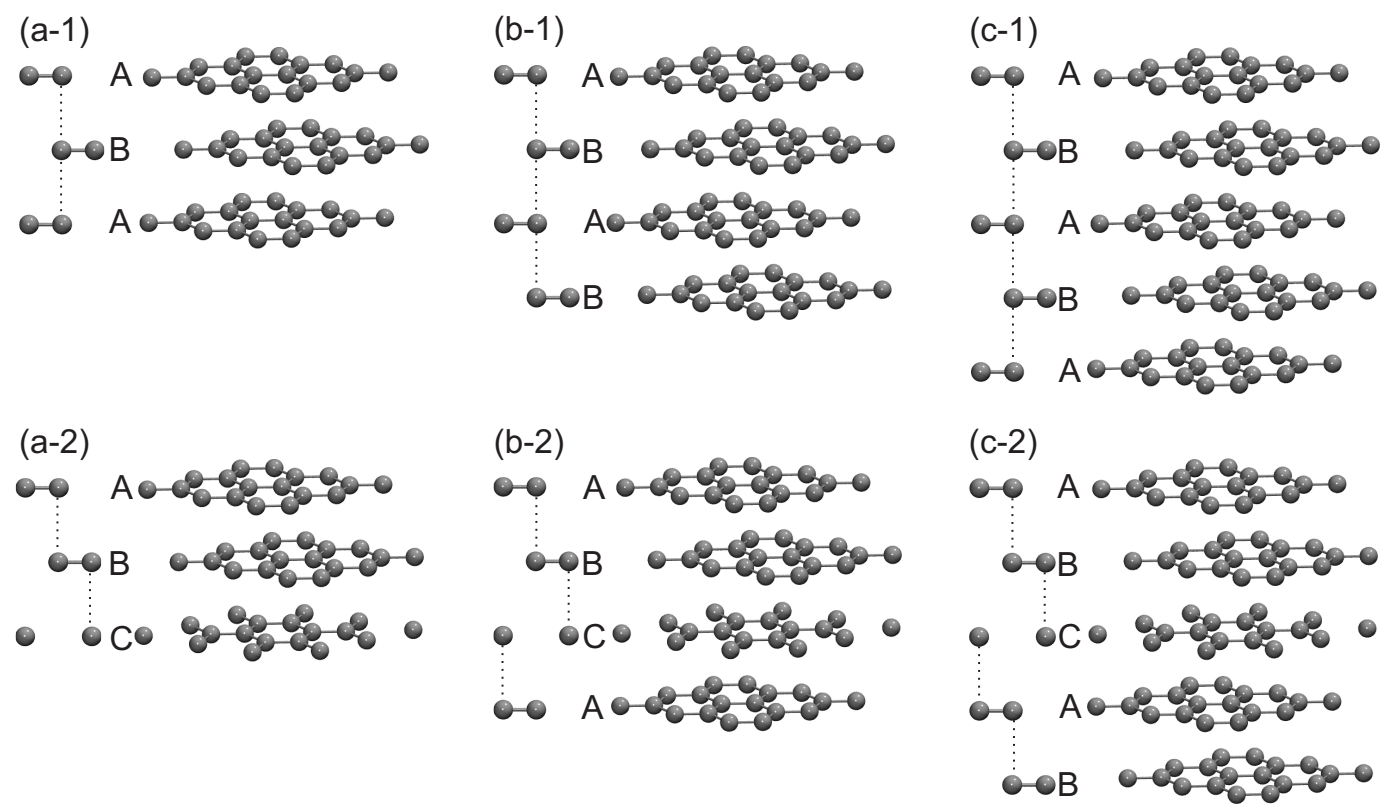

Figura 5.1: Representação esquemática dos empilhamentos Bernal $(\mathrm{AB})$ e Romboédrico $(\mathrm{ABC})$ para: (a-1) Tricamadas AB; (b-1) Tetracamadas AB; (c-1) Pentacamadas AB; (a-2) Tricamdas ABC; (b-2) Tetracamadas ABC; (c-2) Pentacamadas ABC.

Para os sistemas representados na Figura5.1, realizamos cálculos de energia total através da teoria do funcional da densidade como implementando no código SIESTA [90] para determinar a energética dos sistemas. Para o potencial de troca e correlação utilizamos o LDA (CeperleyAlder) [66] e o GGA (Perdew-Burk-Ernzerhof) [91] + Grimme [67], que é uma correção para levar em conta interações de longo alcance como van der Waals ${ }^{4}$. Para as relaxações das geometrias, utilizamos um sampling $30 \times 30$ pontos $k$. Para os cálculos das bandas de energia utilizamos $200 \times 200$ pontos-k, de acordo com o esquema de Monkhorst-Pack [71]. A estrutura

\footnotetext{
${ }^{4}$ Utilizamos esta aproximação que leva em conta interações de van der Waals pois é sabido que o LDA falha na descrição de interações de longo alcance, mas descreve muito bem interações de curto alcance fornecendo com uma boa acurácia a estrutura eletrônica do estado fundamental do sistema, bem como e a energia de ligação entre camadas, que fica em boa concordância com o experimento.
} 
Tabela 5.1: Energias de formação e distâncias de equilíbrio das FLG

\begin{tabular}{c|cc|cc}
\hline \hline Sistema & LDA & \multicolumn{2}{|c}{ GGA+VDW } & \\
\hline \hline & $E_{f}(e V)$ & $d(\AA)$ & $E_{f}(e V)$ & $d(\AA)$ \\
\hline \hline ABA & -0.152 & 3.217 & -0.227 & 3.294 \\
ABC & -0.151 & 3.227 & -0.227 & 3.303 \\
\hline ABAB & -0.228 & 3.216 & -0.348 & 3.308 \\
ABCA & -0.227 & 3.221 & -0.349 & 3.290 \\
\hline ABABA & -0.303 & 3.220 & -0.470 & 3.288 \\
ABCAC & -0.303 & 3.220 & -0.469 & 3.265 \\
\hline \hline
\end{tabular}

bidimensional do sistema foi simulada utilizando o parâmetro de rede otimizado para o grafeno e uma separação de $40 \AA$ de vácuo na direção perpendicular às camadas, para evitar interações entre as imagens periódicas nessa direção.

Na tabela 5.1, mostramos as energias de formação para sistemas compostos de 3, 4 e 5 camadas de grafeno, utilizando a aproximação LDA e GGA+vdW. Utilizamos como fonte de átomos de carbono o grafeno. A distância de separação entre as camadas foi otimizada para cada estrutura. Analisando os sistemas em pares, não observamos diferenças significativas entre as energias de formação dos dois tipos de empilhamentos, mostrando que ambos os sistemas são passíveis de existirem. Comparando os funcionais LDA e GGA+VDW, verificamos que este último descreve melhor a distância de separação entre as camadas, que se aproximam mais do grafite, ou seja, $d \approx 3.3 \AA$. Novamente, esta distância é equivalente para ambos os tipos de empilhamentos. Esses resultados são consistentes com a observação de estruturas romboédricas no grafite natural e sintetizado [92], e também observado experimentalmente nas FLG $[83,86,93]$.

\subsection{Importância do empilhamento na estrutura eletrônica do sistema}

Seguindo com o estudo das FLG, determinamos qual a influência do tipo de empilhamento na estrutura eletrônica. Antes de entrarmos de fato na análise sobre a influência do tipo de empilhamento na estrutura eletrônica do sistema, apresentamos na figura 5.2, as estruturas de bandas para as tricamadas, tetracamadas e pentacamadas, em ambos os empilhamentos, considerando as aproximações LDA e GGA+vdW. Podemos observar que essas estruturas de bandas não possuem nenhuma diferença significativa. Dessa maneira, ambas as aproximações 
fornecem os mesmos resultados, do ponto de vista da estrutura eletrônica, sendo justificável o uso da aproximação LDA para os outros cálculos.
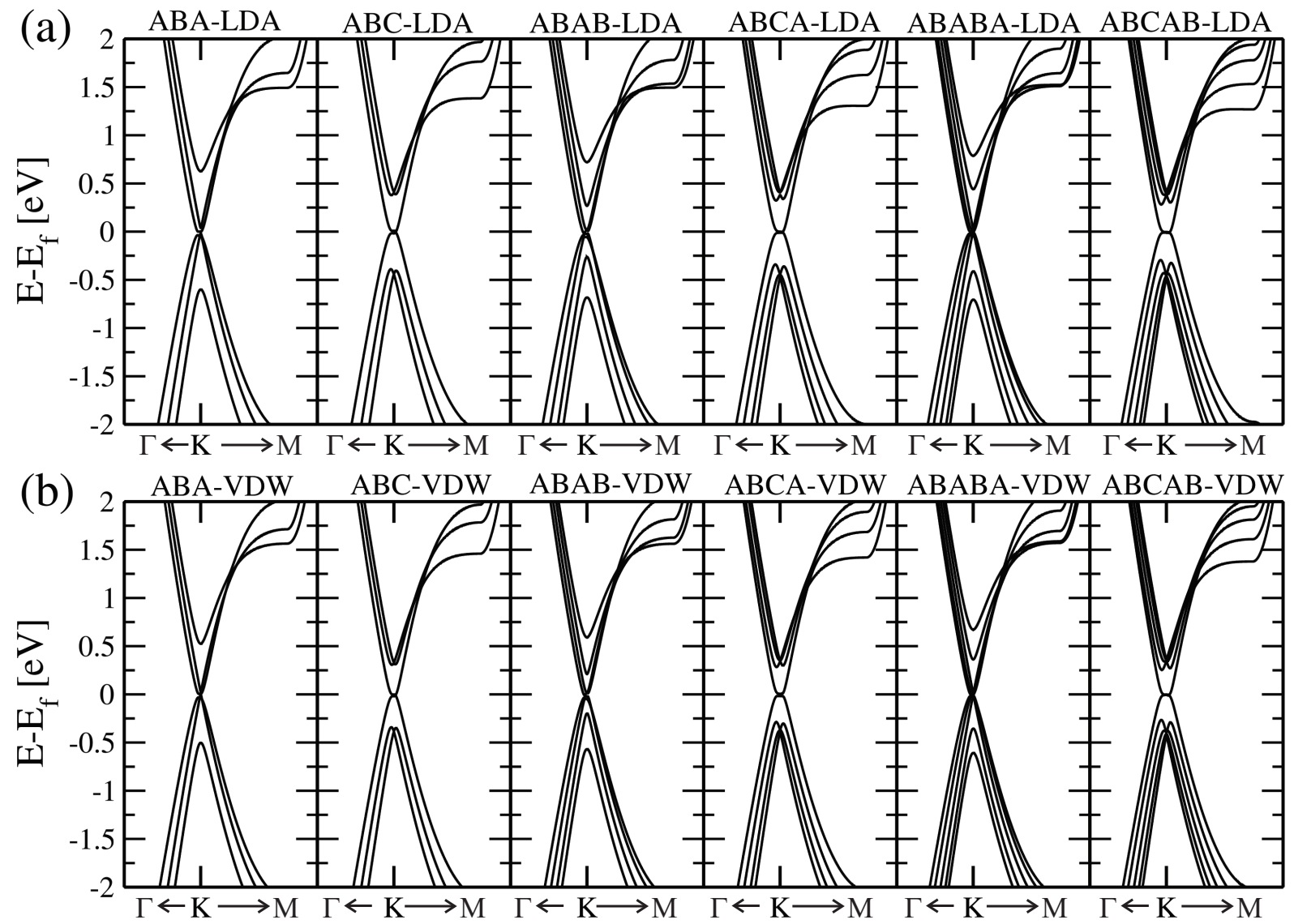

Figura 5.2: Estrutura de bandas para as tricamadas $\mathrm{ABA}$ e $\mathrm{ABC}$; tetracamadas $\mathrm{ABAB}$ e $\mathrm{ABCA}$; e pentacamadas ABABA e ABCAB; utilizando (a) LDA e (b) GGA+vdW.

Continuando com o o entendimento da influência do empilhamento na estrutura eletrônica das multicamadas de grafeno, utilizamos um campo elétrico externo aplicado perpendicularmente ao sistema. Na figura 5.3, apresentamos os resultados para os sistemas em questão, na presença de um campo elétrico externo perpendicular às camadas, de intensidade $3 \mathrm{~V} / \mathrm{nm}$. É aparente nestes resultados em quais sistemas um gap de energia pode ser induzido através da aplicação do campo. Este efeito tem uma forte dependência com o empilhamento das camadas nas FLG. Todos os empilhamentos ABC, figura5.3(b),(d) e (f) podem ser convertidos em semicondutores através da aplicação do campo elétrico externo, enquanto que as estruturas $\mathrm{AB}$, figura5.3(a),(c) e (e), permanecem semimetálicos.

Examinando a estrutura de bandas dos empilhamentos $\mathrm{AB}$ (ABA, ABAB e ABABA) na figura 5.3(a,c,e), podemos observar um overlap de bandas induzidos pelo campo elétrico em 


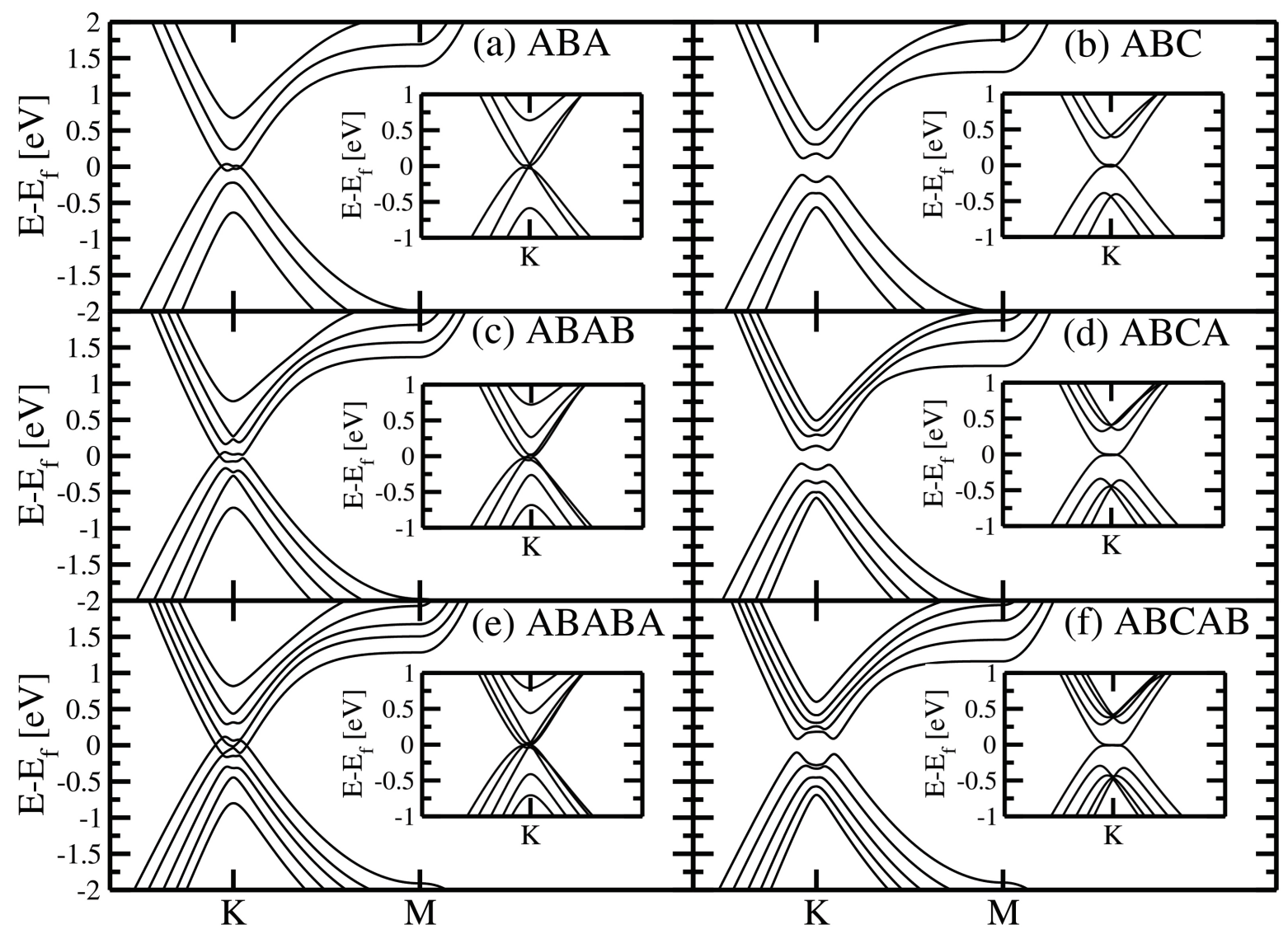

Figura 5.3: Estruturas de bandas para as FLG na presença de um campo elétrico externo de $3 \mathrm{~V} / \mathrm{nm}$ para as: Tricamadas (a) ABA e (b) ABC; Tetracamadas (c) ABAA e (d) ABCA; Pentacamadas (e) ABABA e (f) $A B C A B$. Nos insets de cada figura, temos a estrutura de bandas para cada sistema sem a presença de campo.

todas as estruturas. Esses resultados estão em concordância com resultados já obtidos na literatura a partir de cálculos tight-binding [34,77,79].

Para explicar o overlap das bandas na presença de um campo elétrico, é útil tratar as FLG com empilhamentos $\mathrm{AB}$, como uma combinação de uma única camada grafeno e bicamadas de grafeno, como mostrado na figura 5.4(c,f). Em primeiro lugar, observa-se que, ao contrário dos empilhamentos $\mathrm{AB}$ com um número par de camadas 5.4(a,b,c) aqueles com um número ímpar de camadas 5.4(d,e,f) sempre possuem uma degenerescência de energia no ponto $\mathrm{K}$, independentemente da intensidade do campo elétrico. Esta degenerescência independente do campo no ponto $\mathrm{K}$, assemelha-se com a propriedade eletrônica de uma única camada de grafeno, originada a partir da camada média nas FLG com número ímpar de camadas. Esta camada intermediária, possui uma interação simétrica com as camadas superiores e inferiores, onde esta deixa o átomo de ligação na camada do meio com uma energia on-site inalterada, fazendo-a se 


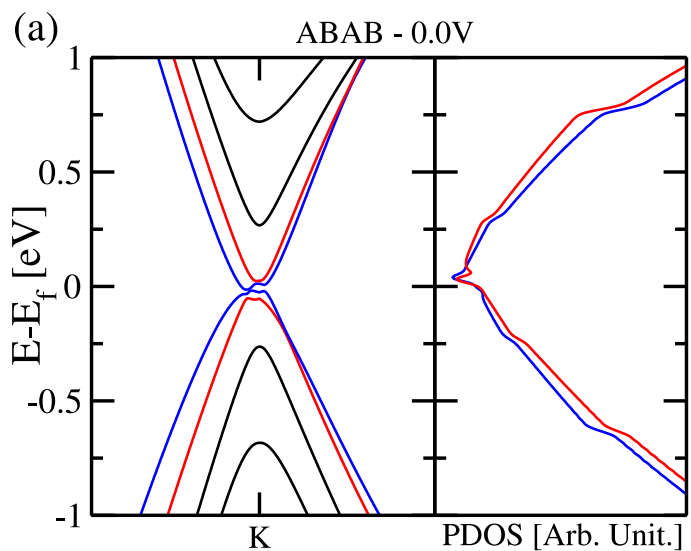

(b)
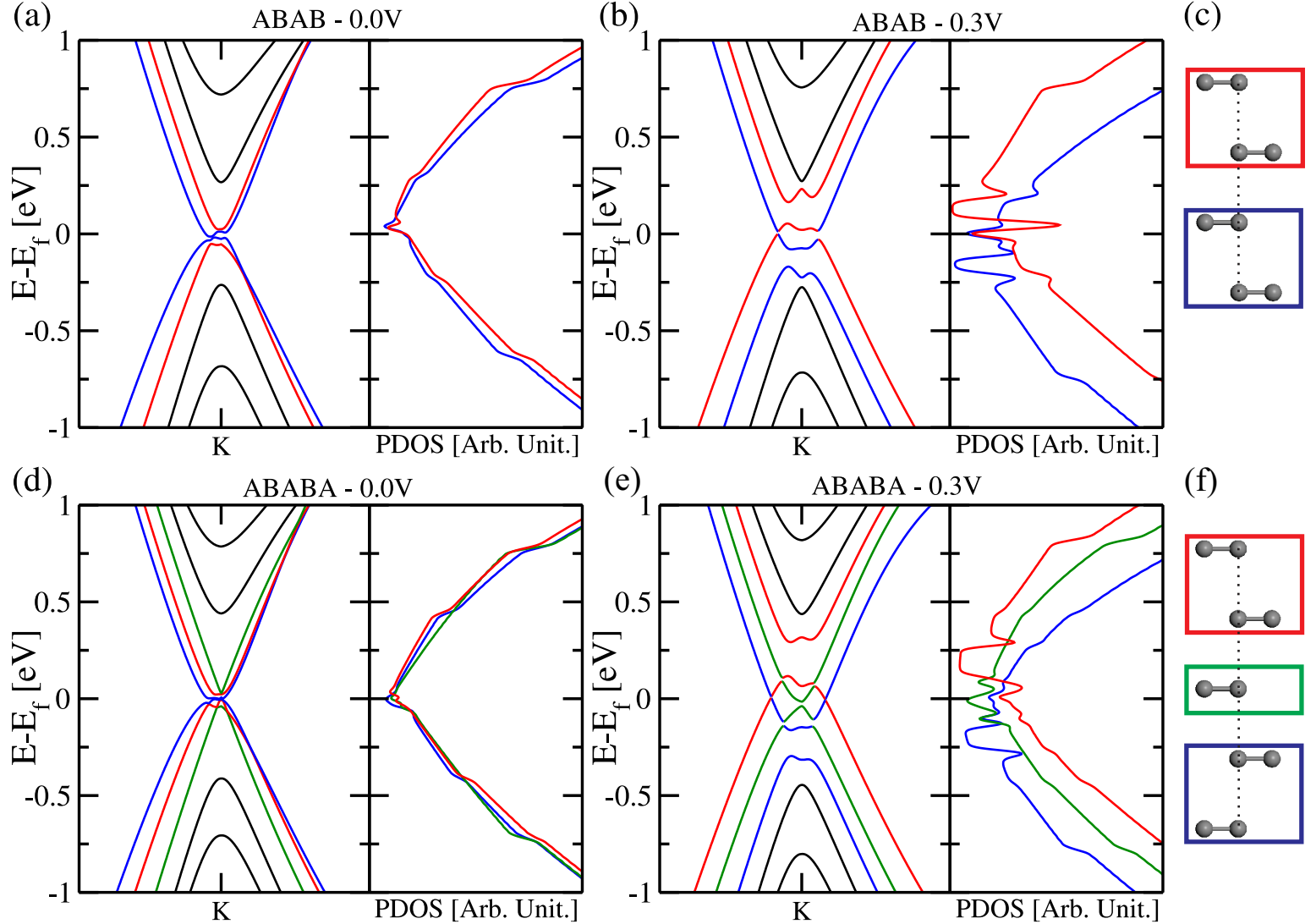

(f)

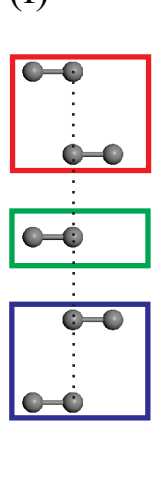

Figura 5.4: Estrutura de bandas para as FLG no empilhamento de Bernal perto do ponto K. Em: (a) Tetracamada - campo elétrico nulo; (b) Tetracamada - campo elétrico $0.3 \mathrm{~V} / \mathrm{nm}$; (c) Esquema de divisão dos subsistemas da tetracamada. Em: (d) Pentacamada - campo elétrico nulo; (e) Pentacamada - campo elétrico $0.3 \mathrm{~V} / \mathrm{nm}$; (f) Esquema de divisão dos subsistemas da pentacamada.

comportar como uma única camada de grafeno isolada. Isso fica mais evidente se observarmos o sistema com 5 camadas ABABA na figura 5.4(d,e,f). Esta análise também explica a presença de férmions de Dirac em sistemas de menos camadas como a tricamada ou mais camadas com empilhamentos $\mathrm{AB}$ e um número ímpar de camadas [81].

Para ficar mais claro essa separação entre monocamadas e bicamadas, podemos reagrupar algumas linhas da estrutura de bandas, onde observamos estruturas similares as bicamadas deformadas por um campo elétrico. Tal fenômeno pode ser explicado de forma eficaz se extrairmos uma série de subsistemas análogos às bicamadas de grafeno no empilhamento $\mathrm{AB}$ das FLG, observando que a interação dos subsistemas vizinhos afeta apenas os estados ligantes e anti-ligantes e tem pouco impacto sobre os estados isolados em cada camada. Como os estados ligantes e anti-ligantes (linhas pretas na figura 5.4) são separados e distantes do nível de Fermi, é realmente a estrutura de banda dos estados isolados (linhas coloridas) que determina se pode 
haver um gap induzido por um campo elétrico. Na figura 5.4(c,f), os subsistemas são delineados por caixas com cores diferentes e cada caixa contém um subsistema que gera a sub-banda com a mesma cor na estrutura de bandas global. O campo elétrico aplicado não só quebra a degenerescência dentro de cada sub-banda das bicamadas, mas também divide essas sub-bandas afastando-as umas das outras. Podemos ver claramente na estrutura de banda que o efeito do campo elétrico, que leva a uma transferência de carga entre as camadas do sistema, conduz inevitavelmente a uma sobreposição das bandas de condução de energia-baixa com as bandas de valência em subsistemas de energia elevada. Com um novo aumento da intensidade de campo, ocorre um aumento da sobreposição das bandas, em vez de criar um gap. Resumindo, a ausência de um gap induzido por um campo elétrico perpendicular aos empilhamentos $\mathrm{AB}$ das FLG com um número camadas par, é atribuído à sobreposição das bandas dos estados isolados pertencentes à diferentes subsistemas de bicamadas. A ausência de um gap nos empilhamentos $\mathrm{AB}$ com um número ímpar camadas é atribuída à sobreposição das banda dos estados das bicamadas e à banda (no ponto K) do subsistema constituído pela monocamada isolada no meio do sistema. Com base neste argumento, sistemas do tipo Bernal, com mais de duas camadas, nunca apresentarão um gap controlável por um campo elétrico.

Quanto ao empilhamento ABC das FLG, o campo elétrico aplicado tem um efeito completamente diferente. Cada célula unitária das N-ABC camadas, pode ser considerada como uma combinação de N-1 dímeros ligantes (sistema circulado pela linha preta na figura 5.5(c,f)) e 2 átomos isolados anti-ligantes localizados em cada superfície das FLG (átomos circulados pelas linhas em azul na figura 5.5(c,f). A campo zero, os dois átomos isolados em cada lado da superfície (em azul), possuem uma energia local idêntica, gerando um par de bandas de valência e condução, com uma degenerescência de energia no ponto $K$, como pode ser visto nas linhas em azul nas figura 5.5(a,d). De maneira equivalente, os átomos pertencentes a cada dímero, dentro das camadas (em preto) interagem uns com os outros, gerando dois estados degenerados no ponto K, formando um conjunto de estados ligantes e antiligantes (linhas pretas nas bandas), que são afastados uns dos outros através de uma intensidade que é proporcional à interação de hopping entre os pares dos dímeros. Portanto, a degenerescência dos estados dos átomos isolados (em azul) é responsável pela ausência de gap quando não existe campo aplicado. Quando um campo elétrico perpendicular é aplicado, é introduzida uma diferença de potencial entre estes dois átomos, quebrando a degenerescência na energia no ponto K. Dessa maneira, ocorre 


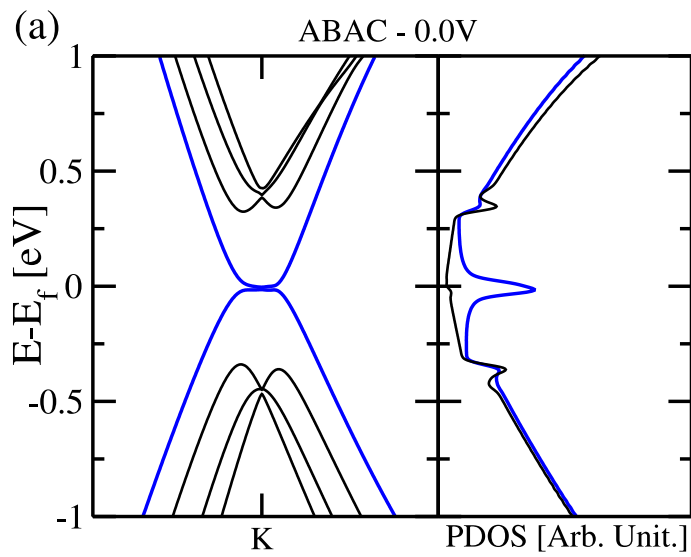

(b)

(c)
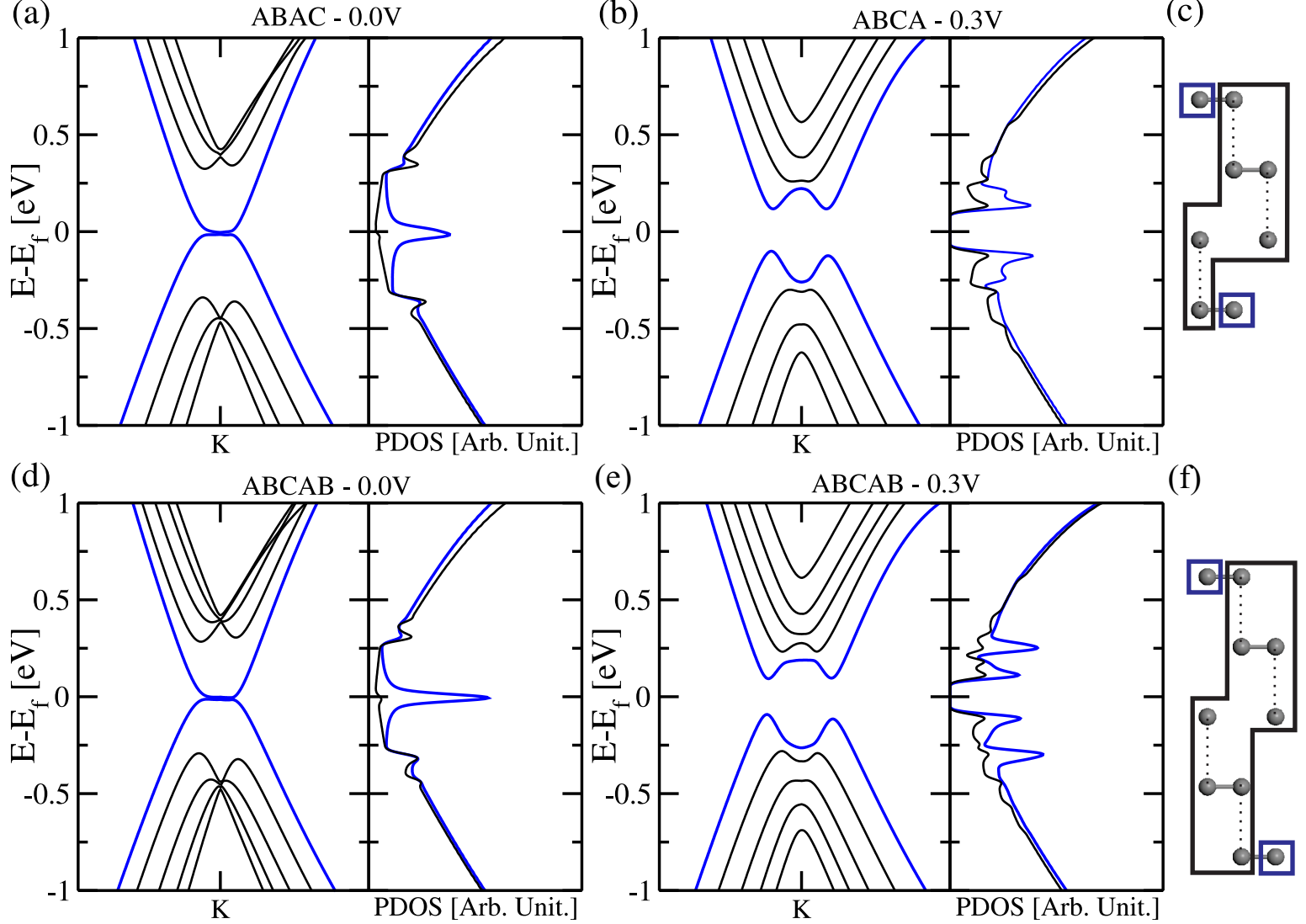

(e)

$\mathrm{ABCAB}-0.3 \mathrm{~V}$

(f)

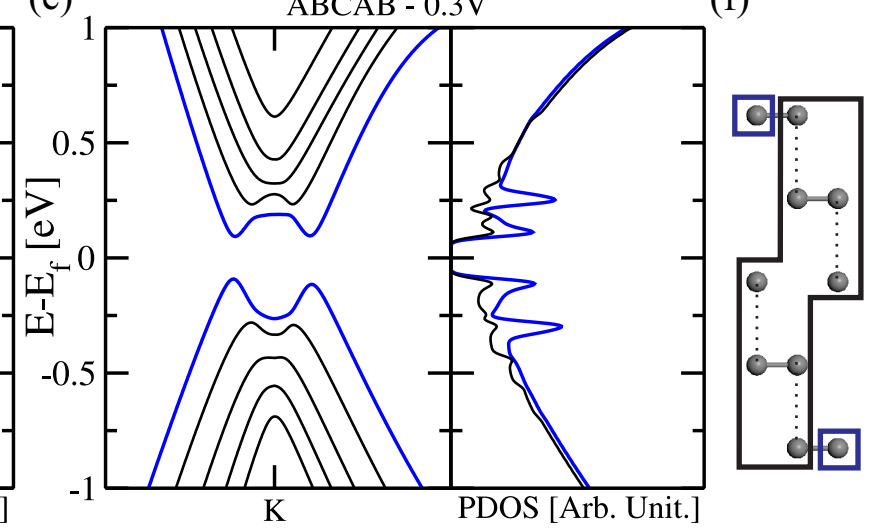

Figura 5.5: Estrutura de bandas para as FLG no empilhamento romboédrico perto do ponto K. Em: (a) Tetracamada - campo elétrico nulo; (b) Tetracamada - campo elétrico $0.3 \mathrm{~V} / \mathrm{nm}$; (c) Esquema de divisão dos subsistemas da tetracamada. Em: (d) Pentacamada - campo elétrico nulo; (e) Pentacamada - campo elétrico $0.3 \mathrm{~V} / \mathrm{nm}$; (f) Esquema de divisão dos subsistemas da pentacamada.

a abertura de um gap de energia que pode ser controlado através do campo elétrico externo (como nas bicamadas de grafeno).

Na figura 5.6, mostramos o valor do gap induzido em função do campo elétrico externo para as FLG-ABC consideradas em nossos cálculos, e também para a bicamada de grafeno para efeitos de comparação. Observamos que o valor do gap induzido aumenta da bicamada para a tricamada. Entretanto, este gap volta a ficar menor a partir da tetracamda. Para entender esta diferença, podemos notar que a aplicação de um campo elétrico perpendicular não só quebra a degenerescência dos átomos isolados (em azul na figura 5.5), mas também gera uma diferença de potencial ao longo do eixo na direção do campo, gerando um shift nas bandas de energia desses átomos e uma expansão em série dos estados ligantes e anti-ligantes longe de sua forma degenerada. Com o aumento da intensidade do campo, os estados ligantes e anti-ligantes dos dímeros, são deslocados para próximos ao nível de Fermi, onde estes estados irão se misturar 


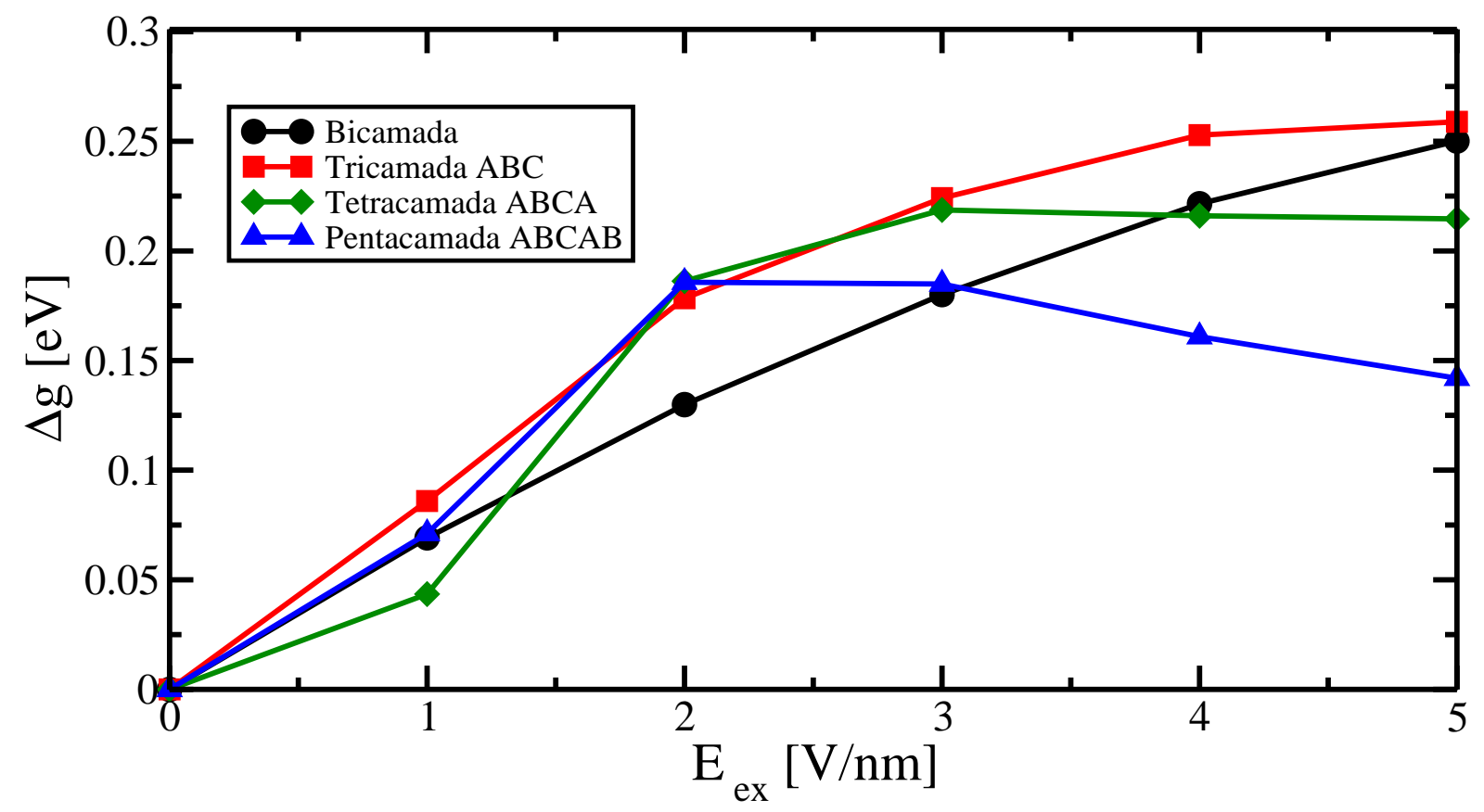

Figura 5.6: Gap de energia em função do campo elétrico externo para as bicamadas, tricamadas, tetracamadas e pentacamadas, no empilhamento ABC.

com as bandas dos átomos isolados de superfície, limitando assim a separação dessas bandas, e consequentemente limitando a abertura do gap de energia, e até mesmo diminuindo este valor, como observamos na figura 5.6. Assim, dentro do ponto de vista de uma aplicação desses sistemas de FLG em um dispositivo de efeito de campo, quanto mais camadas forem utilizadas, menor será os valores dos gaps de energia que podem ser induzidos pelos potencias de gate, limitando dessa forma a performance e eficiência do dispositivo.

\subsection{Influência do empilhamento nas propriedades de trans- porte das FLG}

Nesta seção iremos estudar qual a influência do empilhamento nas propriedades de transporte das multicamadas de grafeno. Para isso, utilizamos FLG compostas de 3 até 12 camadas em ambos os empilhamentos, Bernal e romboédrico. Além da importância do empilhamento, estudaremos qual a influência do número de camadas no transporte. Na figura 5.7, mostramos um esquema dos sistemas utilizados nos cálculos de transporte. Para as propriedades de transporte utilizamos o formalismo de funções de Green fora do equilíbrio acoplado com a teoria do funcional da densidade, conforme descrito na seção 3.2. Utilizamos uma base dupla- $\zeta$, com 
$300 R y$ de mesh cut-off e 10000 pontos $k_{\perp}$ transversais à direção de transporte. Ressaltamos, aqui, que tanto a região de espalhamento como os eletrodos são compostos do mesmo material.

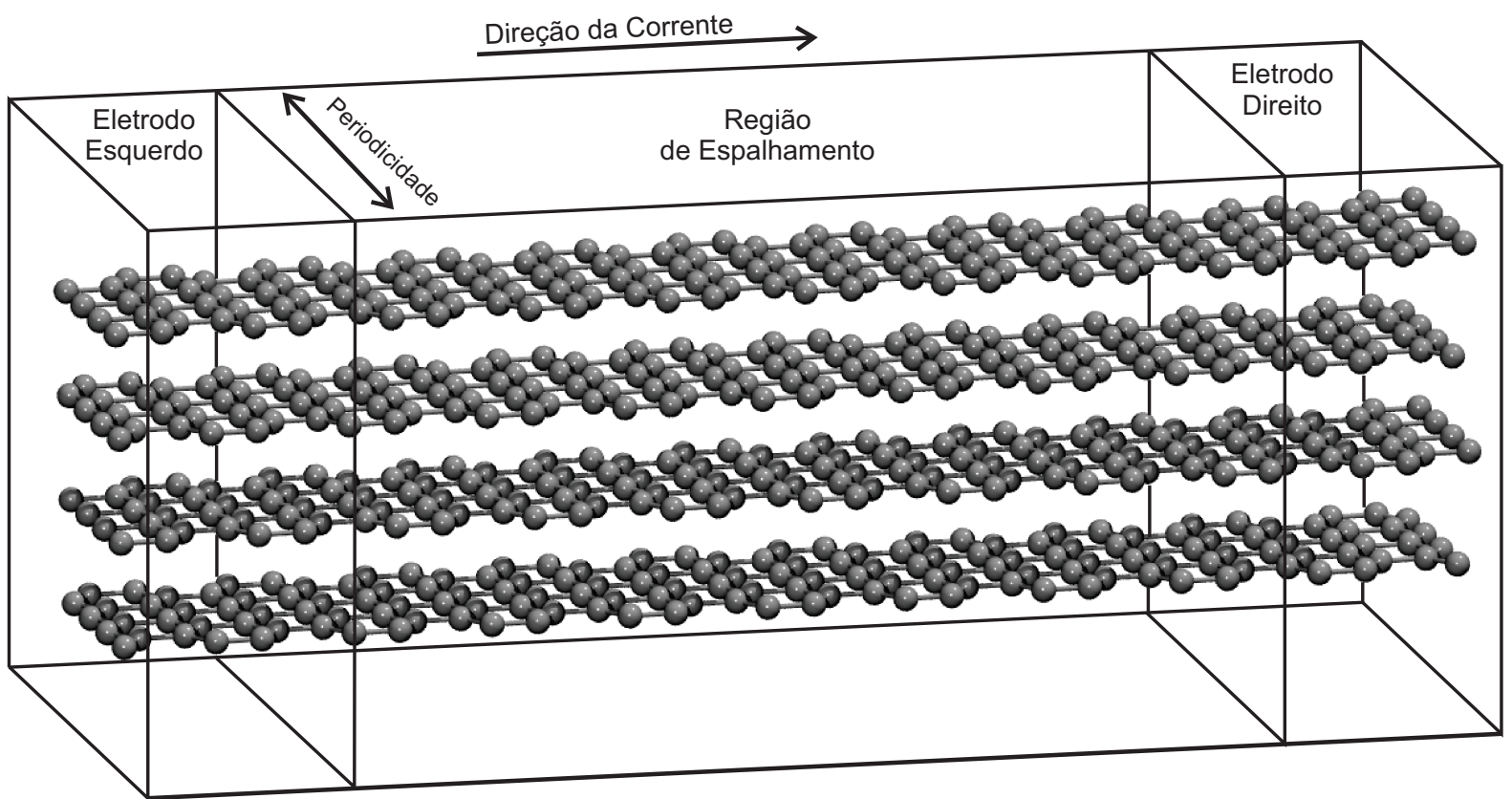

Figura 5.7: Representação esquemática do sistema utilizado nos cálculos de transporte das FLG.

Na figura 5.8, mostramos a estrutura de bandas, densidade de estados (DOS), transmitância $(T(E))$ e densidade local de estados (LDOS), para as FLG, indo da tricamada até a hexacamada, para os empilhamentos de Bernal $(\mathrm{AB})$ e romboédrico $(\mathrm{ABC})^{5}$. Primeiramente, podemos notar que somente com a mudança da seqüencia de empilhamento, a estrutura eletrônica e as propriedades de transporte são fortemente modificadas. Analisando o empilhamento de Bernal, quadros superiores na figura $5.8(\mathrm{a}, \mathrm{b}, \mathrm{c}, \mathrm{d})$, podemos notar que a transmitância do sistema é muito semelhante à densidade de estados, como era de se esperar, pois estamos realizando cálculos de transporte de um sistema pristino. Além disso, olhando para a LDOS (calculada na região de energia indicada pela linha vermelha utilizando uma janela de integração de $3 m e V$ ao redor do nível de Fermi), notamos que o transporte se dá por todas as camadas do sistema.

Quando olhamos para o caso romboédrico, quadros inferiores na figura 5.8(a, b, c, d), o primeiro ponto a ser notado é a existência de um pico na densidade de estados, no nível de Fermi, que não é refletido na transmitância, mesmo tratando-se de uma sistema pristino ${ }^{6}$. Isso

\footnotetext{
${ }^{5}$ Nesta figura apresentamos quatro resultados representativos para os empilhamentos. O restante dos resultados para 7 até 12 camadas apresentam o mesmo comportamento.

${ }^{6}$ Essa característica onde a DOS do sistema não é refletido no transporte, mesmo no caso de um sistema pristino, reforça a necessidade da realização de cálculos de transporte eletrônico para o entendimento do sistema.
} 

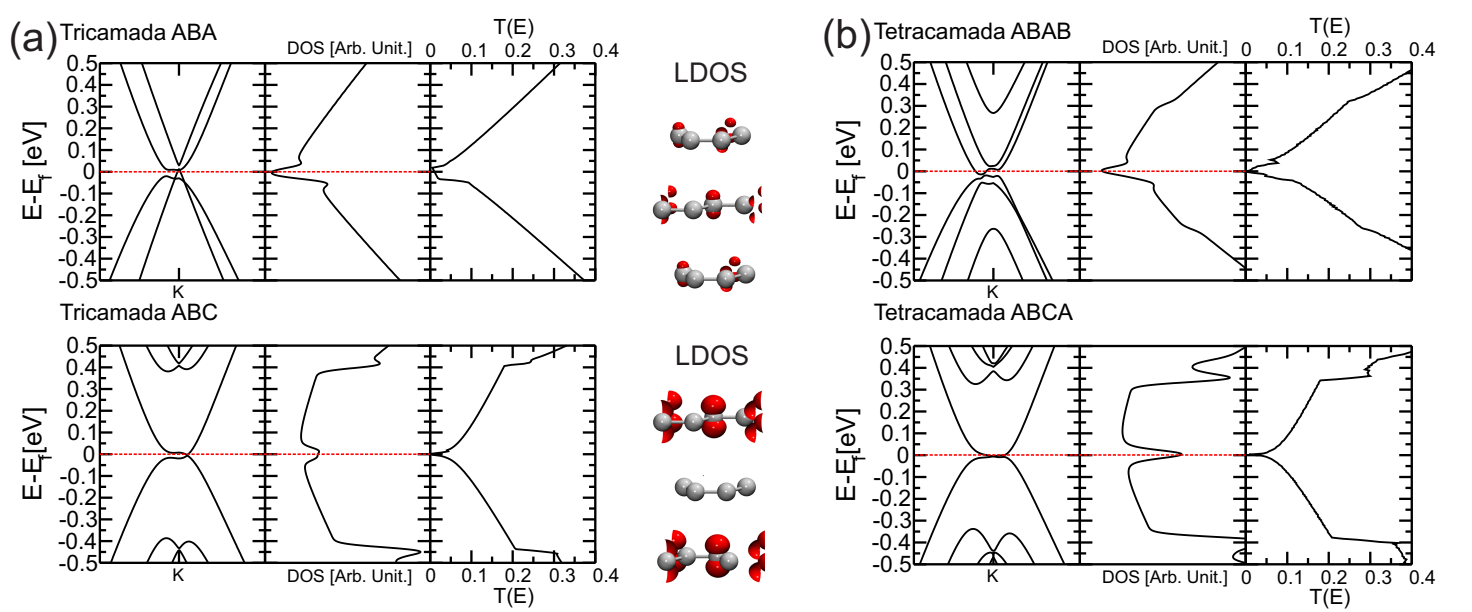

LDOS

sies:

Tetracamada ABCA

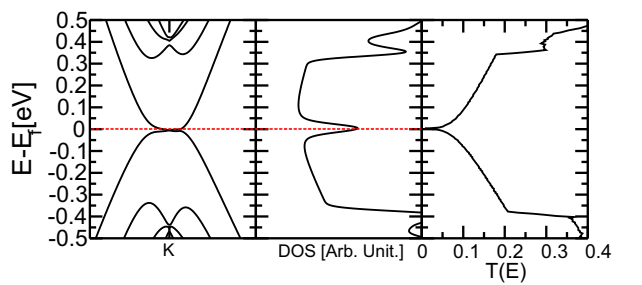

LDOS

cion

00

00

008
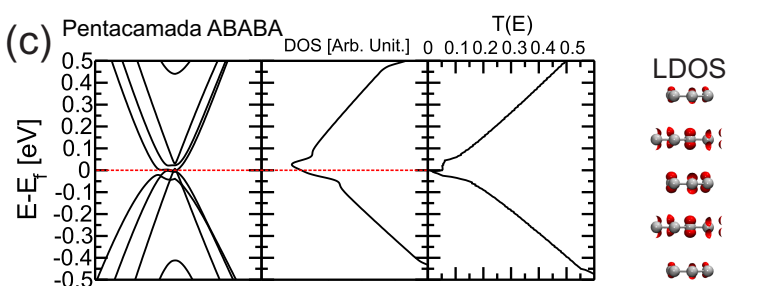

(d)
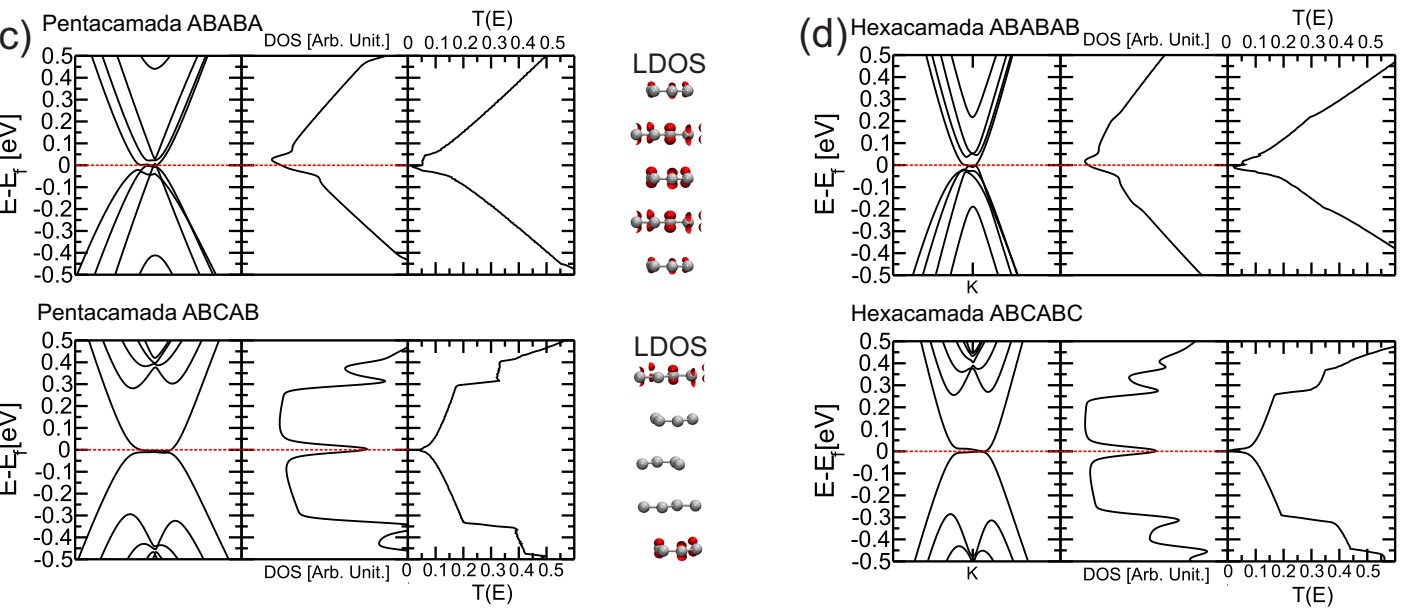

LDOS

ind

$\infty \infty$

$\infty \infty$

000

$\infty-\infty$

Figura 5.8: Estrutura de bandas, densidade de estados (DOS), transmitância $(T(E))$ e densidade local de estados (LDOS), para as FLG: (a) Tricamada; (b) Tetracamada; (c) Pentacamada; (d) Hexacamada. Nos quadros superiores em cada figura temos o empilhamento de Bernal e nos quadros inferiores o empilhamento romboédrico.

ocorre pois trata-se de um estado localizado, somente no ponto $K$, proveniente dos átomos das camadas mais externas do sistema, e dessa maneira não contribui para o transporte. Notamos também, através da LDOS do sistema, que diferentemente do caso Bernal, o transporte nesse sistema ocorre majoritariamente através da superfície do material, onde as camadas interiores das FLG não contribuem de forma significativa na região do nível de Fermi.

Para elucidar se as propriedades de transporte são majoritariamente pela superfície, plotamos na figura 5.9, a LDOS para a hexacamada no empilhamento romboédrico, utilizando outros intervalos de integração da LDOS (0.2 eV e $0.6 \mathrm{eV})$. Da figura 5.9(b), observamos que quando aumentamos a janela de energia para regiões mais distantes do nível de Fermi, por exemplo $0.2 \mathrm{eV}$ de integração, as camadas interiores do material começa a apresentar uma contribuição significativa. Quando a janela de energia começa a englobar os estados correspondentes ao 


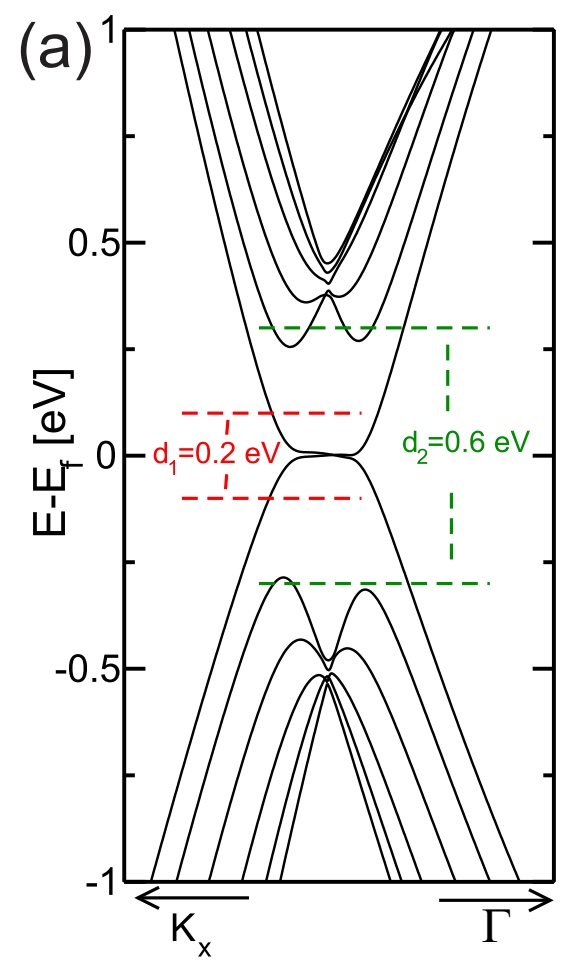

(b) $d_{1}=0.2 \mathrm{eV}$

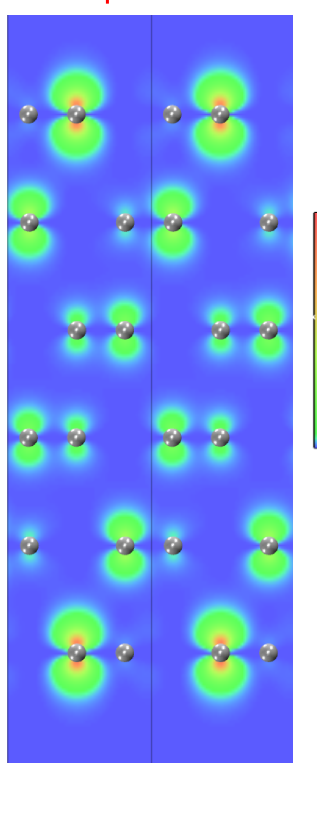

(c)

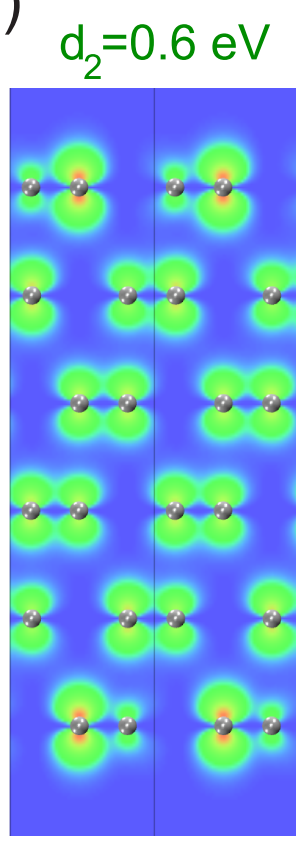

Figura 5.9: (a) Estrutura de bandas para hexacamada no empilhamento romboédrico mostrando os intervalos de integração para a densidade local de estados. (b) Densidade local de estados para $0.2 \mathrm{eV}$ de integração ao redor do nível de Fermi. (c) Densidade local de estados para 0.6 eV de integração ao redor do nível de Fermi.

pares que estão interagindo entre si, o transporte será pelo interior da FLG no empilhamento romboédrico, como observamos na figura 5.9(c). Este comportamento também foi observado quando variamos o número de camadas. Na figura 5.10, mostramos a LDOS para a tricamada, tetracamada e pentacamada onde podemos observar que para esses sistemas existe também a contribuição das camadas interiores ao material, ficando evidente que quanto menos camadas maior é essa contribuição.

Continuando com o entendimento das propriedades de transporte das FLG, na figura 5.11 mostramos a corrente fonte dreno $\left(I_{d s}\right)$, em função da voltagem fonte-dreno $\left(V_{d s}\right)$, à temperatura ambiente, para os sistemas de 3 a 12 camadas para ambos os empilhamentos. Primeiramente, podemos notar que para todos os sistemas considerados, as curvas $I_{d s} \times V_{d s}$ apresentam um comportamento linear, indicando um regime ôhmico do sistema nesse intervalo de tensões de bias. Um outro ponto, é que a corrente para o empilhamento romboédrico, com 3 e 4 camadas, é maior que a corrente no empilhamento de Bernal.

Quando olhamos para as variações nas correntes, para o caso romboédrico e Bernal aumen- 


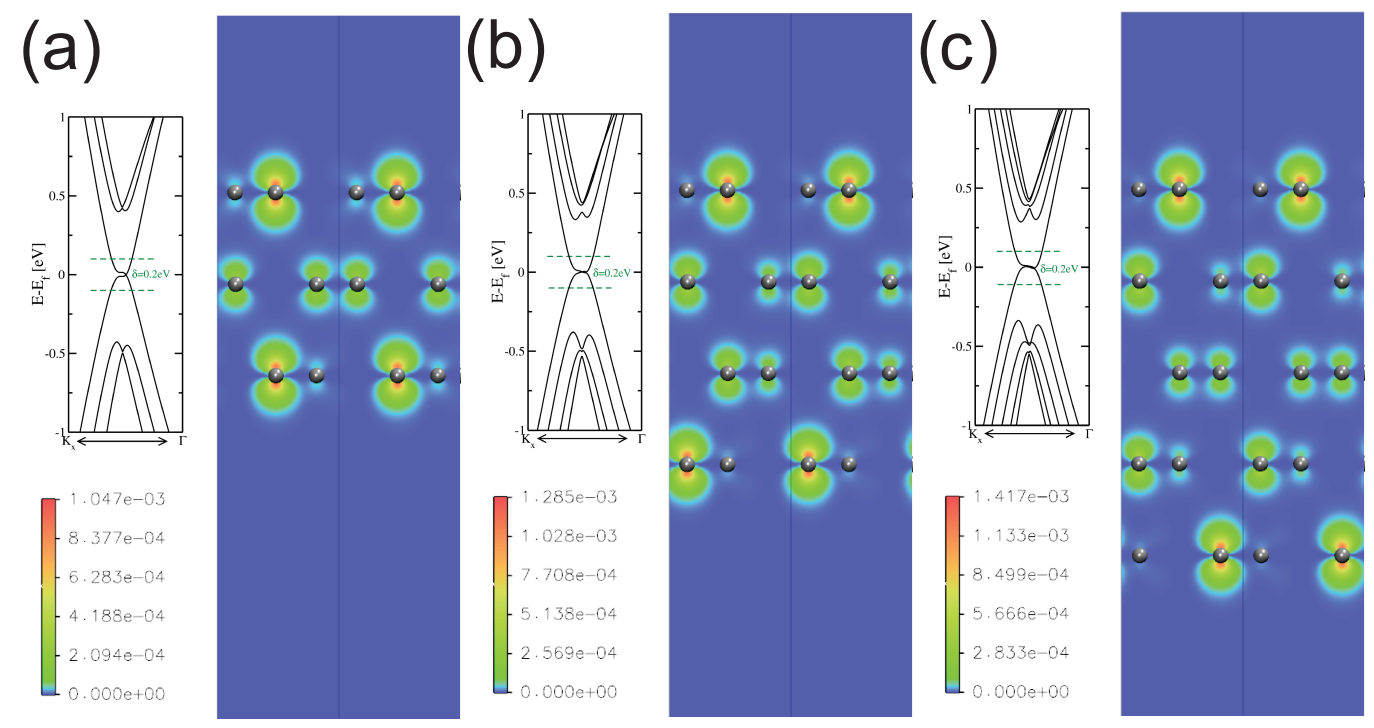

Figura 5.10: Densidade local de estados - LDOS - para (a) tricamada; (b) tetracamada; (c) pentacamada. $\mathrm{O}$ intervalo de integração foi de $0.2 \mathrm{eV}$ ao redor do nível de Fermi.
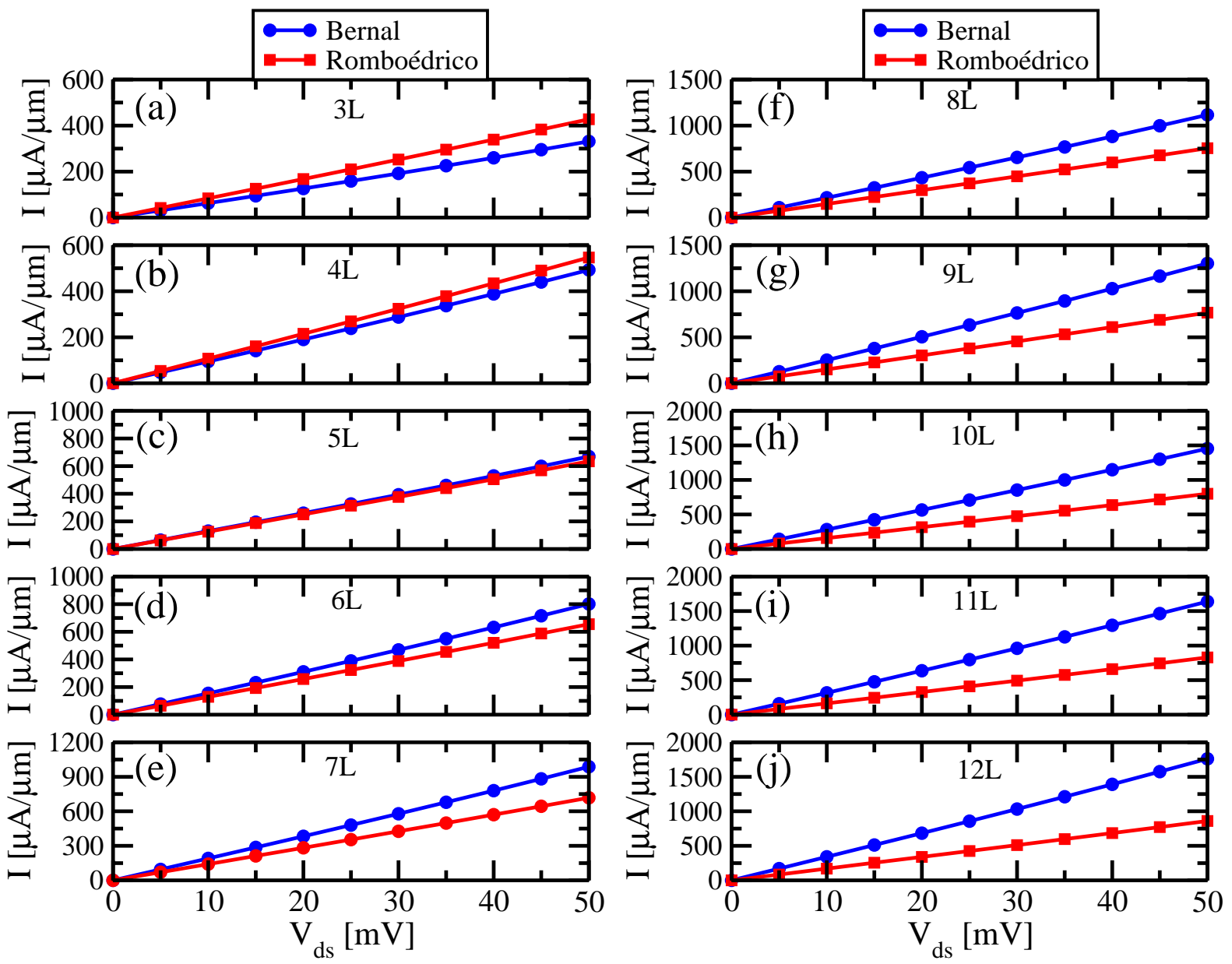

Figura 5.11: Correntes fonte-dreno para as FLG de 1 até 12 camadas para as FLG em ambos os empilhamentos, respectivamente. 
tando o número de camadas no sistema, observamos que no caso romboédrico, a corrente varia muito pouco de 3 a 12 camadas, onde a ordem de grandeza da corrente não se altera, indo de aproximadamente $400 \mu \mathrm{A} / \mu \mathrm{m}$ para 3 camadas a $\approx 900 \mu \mathrm{A} / \mu \mathrm{m}$ para 12 camadas, com um de bias $50 \mathrm{mV}$ à temperatura ambiente. Isso ocorre, pois como já observamos anteriormente, o transporte neste tipo de sistema ocorre majoritariamente pela superfície do material, limitando desta maneira o aumento na corrente. Entretanto, o pequeno aumento observado ocorre pois existe um aumento na contribuição de estados devido às camadas interiores do sistema, gerando dessa forma uma pequena diminuição na resistência do material. Ao contrário do caso romboédrico, a corrente para o caso Bernal aumenta mais de uma ordem de grandeza, indo de $\approx 300 \mu \mathrm{A} / \mu \mathrm{m}$ para $\approx 1750 \mu \mathrm{A} / \mu \mathrm{m}$, com um bias de $50 \mathrm{mV}$ à temperatura ambiente. Estes resultados corroboram a interpretação que o transporte no caso romboédrico é majoritariamente por superfície, pois mesmo aumentando o número de camadas no sistema, o número de canais de transporte não é alterado significativamente. Ao contrário, no caso Bernal, onde o transporte se dá através de todas as camadas do sistema, quanto mais camadas forem adicionadas ao sistema, mais canais de transporte serão abertos, e assim a corrente aumenta consideravelmente, diminuindo muito a resistência do sistema.

Para ficar mais claro o entendimento das variações na corrente, podemos olhar para o comportamento da resistência do sistema em função do aumento do número de camadas. Como na região de tensão de bias que estamos tratando, as FLG apresentam um comportamento ôhmico, podemos calcular a resistência do problema como sendo o inverso da inclinação da curva $I_{d s} \times V_{d s}$ apresentadas na figura 5.11. Na figura 5.12(a), mostramos o valor da resistência das FLG em função do número de camadas no sistema. Observamos que para o caso Bernal a resistência possui uma queda grande, corroborando as interpretações que quanto mais camadas são adicionadas no sistema, mais canais de condução são abertos e dessa maneira a resistência diminui consideravelmente. No caso romboédrico, a resistência varia muito pouco, pois nesse tipo de sistema a resistência do material está vinculada a um transporte majoritariamente de superfície, e como vimos, com o aumento do número de camadas devido a penetração de estados nas camadas interiores, temos um pequeno decréscimo na resistência.

Na figura 5.12(b), apresentamos o comportamento da resistência em função do inverso do número de camadas no sistema. Como podemos observar, o sistema com empilhamento de Bernal apresenta um comportamento linear da resistência em função do inverso do número de 

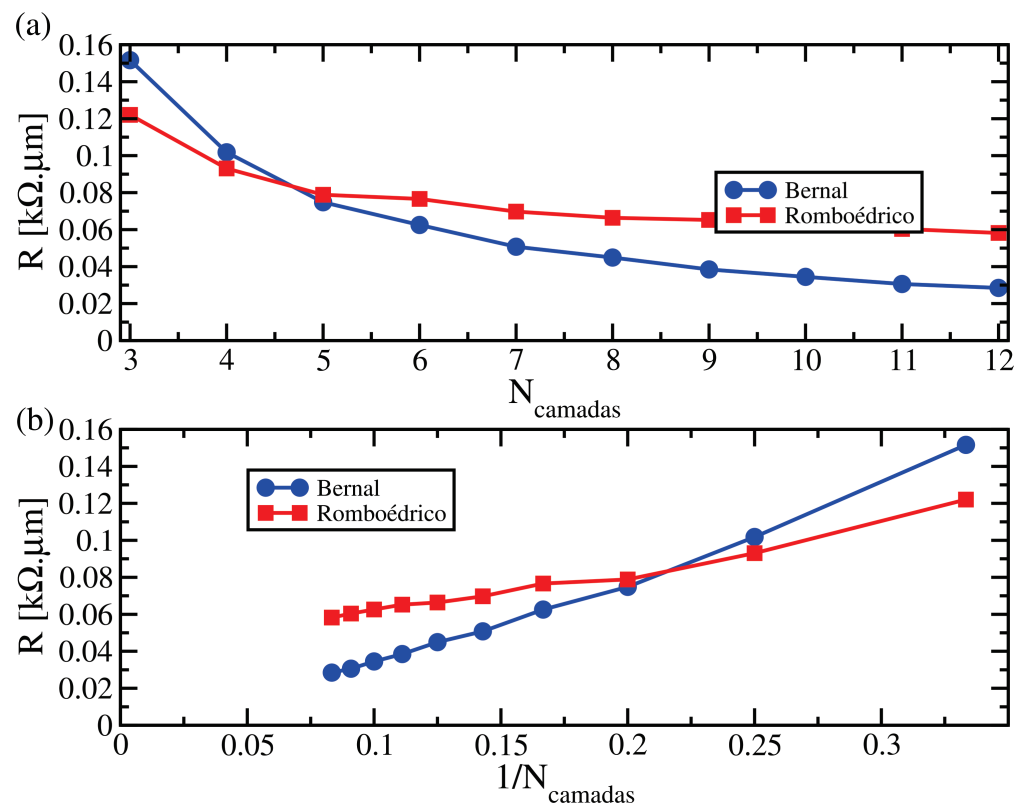

Figura 5.12: (a) Resistência para as FLG em ambos os empilhamentos e em função do número de camadas de grafeno. (b) Resistência para as FLG em ambos os empilhamentos em função do inverso do número de camadas.

camadas, onde através da tendência da curva, quando o número de camadas for para o infinito, a resistência do material irá tender a zero, como era de se esperar. Para o caso romboédrico, ao contrário do caso Bernal, a resistência do material em função do inverso do número de camadas, não aparenta tender a zero quando o número de camadas tende ao infinito.

Um outro ponto importante, como podemos observar na figura 5.12, é que através da resistência obtida para os empilhamentos é possível diferenciar um sistema do outro, ou seja, para 3 e 4 camadas o sistema que apresenta menor resistência é o caso romboédrico, e a partir de 5 camadas os sistemas com empilhamento Bernal apresentam menor resistência. Isso possibilita uma diferenciação entre os dois tipos de empilhamentos através das suas propriedades de transporte.

O mecanismo de transporte nesses sistemas de algumas camadas de grafeno são fortemente dependentes da temperatura [85]. Dessa maneira, o comportamento da corrente e da resistência em função da temperatura, pode também ser utilizado para diferenciação do tipo de empilhamento nas FLG. Nos casos estudados, a variação da temperatura é refletida no potencial químico do reservatório de elétrons do sistema. Para entender a influência da temperatura na corrente para os diferentes empilhamentos, realizamos cálculos de transporte, fixando a voltagem fonte-dreno em $V_{d s}=50 \mathrm{mV}$, para temperaturas indo de $50 \mathrm{~K}$ até $300 \mathrm{~K}$. Na figura 5.13 
mostramos a corrente fonte-dreno, $I_{d s}$, em função da temperatura, para o empilhamento Bernal e romboédrico.
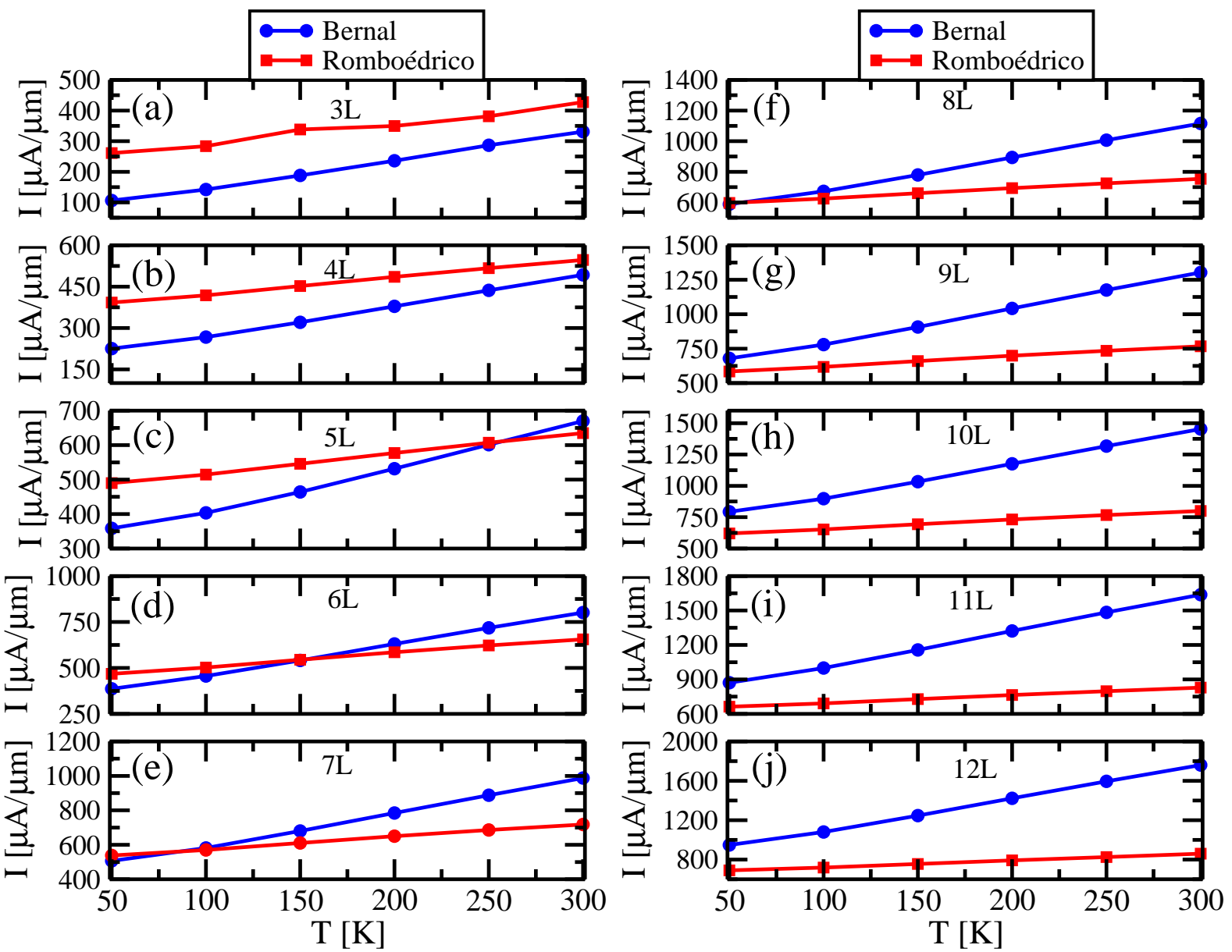

Figura 5.13: Corrente em função da temperatura para os empilhamento de Bernal e romboédrico, de 3 até 12 camadas.

Observando as curvas na figura 5.13, podemos notar que existem 3 classes de sistemas dentro do intervalo de $50 \mathrm{~K}$ até $300 \mathrm{~K}$. O primeiro consiste em 3 e 4 camadas, onde neste intervalo de temperatura o empilhamento romboédrico sempre apresenta a maior corrente. O segundo consiste em 5, 6, 7 e 8 camadas, onde variando-se a temperatura, observa-se uma inversão na corrente predominante. O terceiro consiste em 9, 10, 11 e 12 camadas, onde o empilhamento de Bernal apresenta os maiores valores de corrente. Dessa maneira, é possível utilizar também a temperatura para diferenciar o tipo de empilhamento das FLG.

Para finalizar, podemos entender o comportamento anômalo apresentado pela resistência do sistema com empilhamento romboédrico, observando a figura 5.14. Nesta figura, apresentamos o comportamento das bandas de energia em função do número de camadas de grafeno, 
onde ressaltamos agora a importância dos estados dos sistemas que interagem entre si, ou seja, os estados das camadas interiores à superfície do material. Como podemos observar na figura 5.14(a), com 3 camadas a distância entre esses estados é de aproximadamente $0.8 \mathrm{eV}$ e para um sistema com 23 camadas, figura 5.14(b), o valor da distância diminui para $\approx 0.15 \mathrm{eV}$. O comportamento da medida $\Delta$ em função do número de camadas está na figura 5.14(c). Somente olhando para esta medida, observamos que quando o número de camadas tender ao infinito, estes estados irão tocar o nível de Fermi, e como eles são estados do interior do sistema, a contribuição desta região irá prevalecer, e a resistência do material irá tender ao grafite. Entretanto, devemos lembrar que quando tratamos com as propriedades de transporte do material, existe a presença da função de distribuição de Fermi-Dirac, que aumenta o intervalo de energia ao redor do nível de Fermi que contribui para o transporte. Para isso, delimitamos na figura 5.14(c), por uma linha pontilhada em azul, quando que a largura dessa função de distribuição irá começar a ocupar os estados pertencentes às camadas interiores ao sistema. Assim, através de uma extrapolação da curva, observamos que a partir de aproximadamente 120 camadas, o sistema irá começar a conduzir também por esses canais, e espera-se que a resistência apresentada na figura 5.12(b), tenha uma queda expressiva.
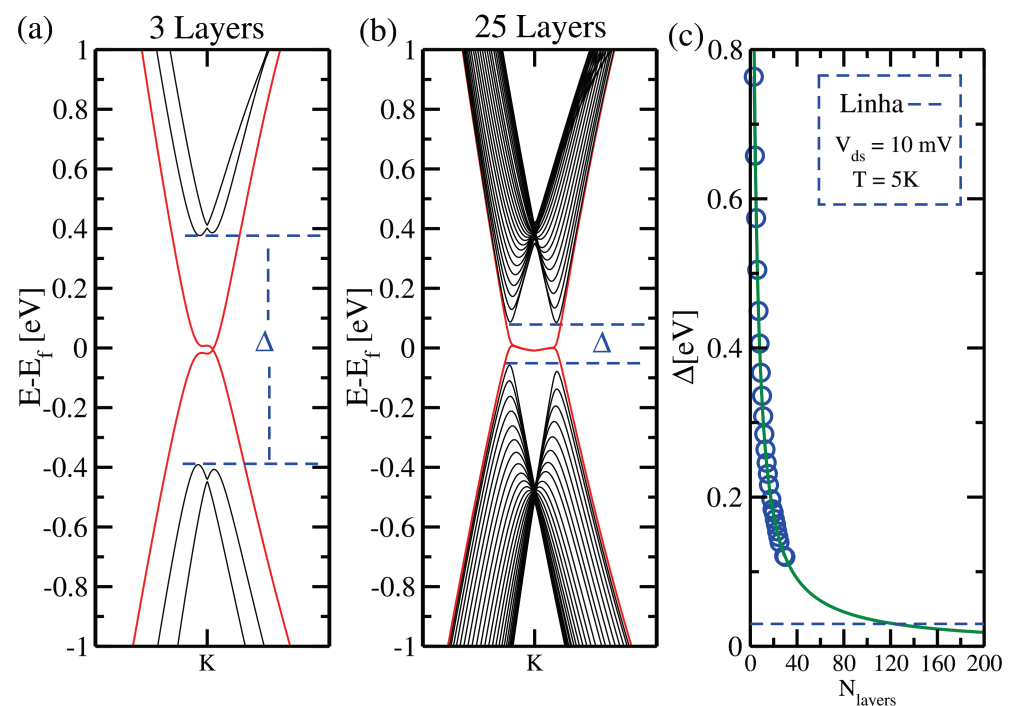

Figura 5.14: (a,b) Comportamento da estrutura de bandas para o caso romboédrico em função do número de camadas no sistema. (c) Comportamento da distância entre os estados do interior da FLG em função do número de camadas. 


\subsection{Conclusões}

As conclusões deste capítulo da tese podem ser enumeradas divididas em duas partes: (i) Estrutura eletrônica do sistema; (ii) Propriedades de transporte.

(i) Propriedades eletrônicas. Para as propriedades eletrônicas do sistema, mostramos que as estruturas de bandas das FLG são fortemente dependentes do empilhamento das camadas. Mostramos que nas FLG com empilhamento de Bernal nunca ocorre abertura de um gap de energia devido a uma sobreposição das bandas. Contrário ao caso Bernal, com o empilhamento romboédrico é possível a obtenção de um gap de energia controlável por um campo elétrico externo, entretanto este gap é limitado pelo número de camadas do sistema, onde quanto mais camadas menor é o gap induzido.

(ii) Propriedades de transporte. Para as propriedades de transporte, mostramos que como na estrutura eletrônica do sistema, elas são fortemente dependentes do tipo de empilhamento nas camadas. No sistema Bernal, o transporte ocorre por todas as camadas do sistema, enquanto que no caso romboédrico o transporte é predominantemente de superfície. Um ponto importante que concluímos é que é possível diferenciar um empilhamento do outro através das propriedades de transporte do sistema, ou seja, simplesmente olhando para a corrente ou resistência do sistema. Mostramos também que é possível utilizar a temperatura para diferenciar um empilhamento do outro. 


\section{Capítulo 6}

\section{Grafeno sobre substratos isolantes de h-BN}

As propriedades eletrônicas do grafeno são muito vulneráveis à condições externas, como corrugações estruturais, átomos ou moléculas adsorvidas, interações com superfícies de substratos, etc. Embora, esse seja um problema para a aplicabilidade do grafeno em dispositivos, este material continua sendo o constituinte principal de novas propostas de nanodispositivos baseados em carbono. Tanto do ponto de vista de uma física fundamental de sistemas bidimensionais, como de uma aplicação direta na engenharia de semicondutores, o estudo da interação do grafeno com outros materiais é muito importante.

Dentre os diversos substratos isolantes, o óxido de silício amorfo, a- $\mathrm{SiO}_{2}$, é o mais utilizado. No a- $\mathrm{SiO}_{2}$, a mobilidade dos portadores é limitada pelo espalhamento dos elétrons com estados de carga superficiais, impurezas carregadas, rugozidades do substrato, etc [24,94-96]. Além disso, perto do ponto de Dirac, devido a uma desordem induzida pelo substrato, o gás de elétrons $2 D$ do grafeno, quebra-se em uma rede não homogênea de elétrons e buracos, como pode ser observado na figura 6.1(b). Outros óxidos outros também foram utilizados juntamente com o grafeno, entretanto, efeitos de superfície semelhantes aos do $\mathrm{SiO}_{2}$ continuam a causar problemas [97-99].

Recentemente, o nitreto de boro hexagonal - h-BN - foi demonstrado ser um forte candidato a substrato para dispositivos baseados em grafeno. Foram obtidos dispositivos de grafeno com uma alta qualidade [25]. O nitreto de boro hexagonal é um isolante com os átomos de boro e nitrogênio ocupando as subredes inequivalentes A e B em uma estrutura hexagonal, como o grafeno. Ele apresenta um gap de energia da ordem de $5 \mathrm{eV}$ [101], uma pequena incompatibilidade com o parâmetro de rede do grafeno, de aproximadamente $1.7 \%$ [102], e pode ser sintetizado 


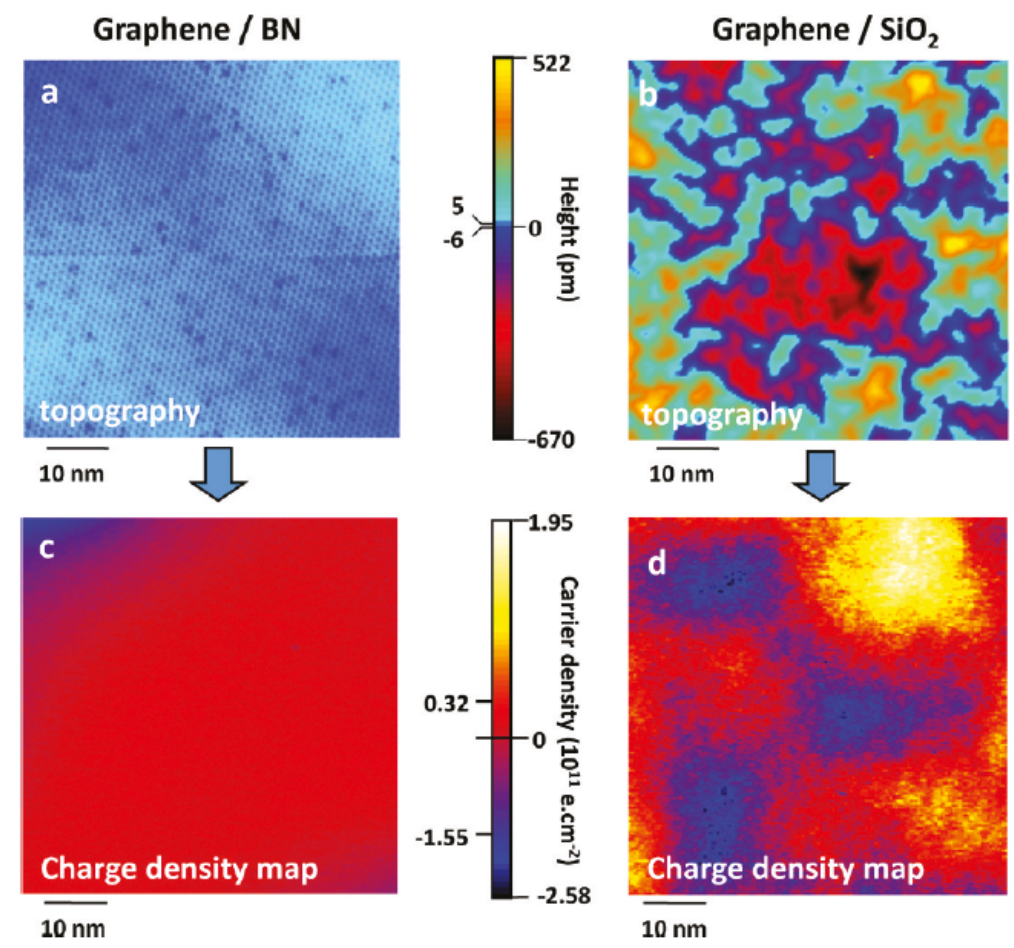

Figura 6.1: Comparação entre a topografia e densidades de carga para grafeno sobre h-BN versus grafeno sobre $\mathrm{SiO}_{2}$.(a,b) Imagens das topografia obtidas por STM para (a) Grafeno/h-BN e (b) Grafeno/SiO 2 . (c,d) Imagens das densidades de carga (a) Grafeno/h-BN e (b) Grafeno/SiO ${ }_{2}$. Nestas iamgens é possível observar a inhomogeneidade da topografia e da densidade de carga no grafeno sobre o $\mathrm{SiO}_{2}$, e como esse problema é praticamente inexistente sobre o substrato de nitreto de boro hexagonal. Figura retirada da referência [100].

com uma alta pureza. Devido a sua forte ligação iônica no plano, a estrutura cristalina planar hexagonal do h-BN é relativamente inerte e espera-se que esteja livre de dangling bonds ou armadilhas de carga na superfície. Além disso, a sua superfície atômica planar deve suprimir as dobraduras no grafeno, que são inerentes à outros substratos [100,103]. Estas qualidades do h-BN podem ser vistas na figura 6.1(a,c). As propriedades dielétricas do h-BN, $\operatorname{com} \epsilon \approx 3-4$ se comparam favoravelmente com as do $\mathrm{SiO}_{2}$, permitindo a sua utilização também como um dielétrico entre o canal e o eletrodo de gate, sem a perda da funcionalidade do grafeno [104]. Além disso, os modos dos fônons ópticos de superfície do h-BN têm energias duas vezes maior do que modos semelhantes no $\mathrm{SiO}_{2}$, sugerindo a possibilidade de uma melhoria no desempenho desses dispositivos em altas temperaturas e altos campos elétricos [105, 106].

Como já foi mencionado, finas camadas de h-BN podem ser obtidas através de exfoliação química. Warner et al. [107] mostraram que o cristal de h-BN apresenta um empilhamento 
$A A^{\prime 1}$, ao contrário do empilhamento $A B$ do grafeno. Entretanto, com a remoção de algumas camadas de $B N$ por irradiação eletrônica ou exfoliação mecânica, pode ocorrer o surgimento de um empilhamento $A B$ na superfície desse material, e não somente o empilhamento $A A^{\prime}$, pois as camadas podem facilmente deslizar uma sobre as outras devido a fraca interação entre elas [108] ${ }^{2}$. Assim, o empilhamento $A B$ pode não ser intrínseco ao cristal de h-BN, mas pode ocorrer na superfície superior e inferior da estrutura em camadas de h-BN [107].

Neste capítulo, estudaremos as propriedades eletrônicas e estruturais das bicamadas de grafeno depositadas sobre uma superfície isolante de Nitreto de Boro Hexagonal. Primeiramente realizaremos um estudo detalhando das propriedades estruturais e eletrônicas deste substrato e depois estudaremos qual o comportamento das bicamadas de grafeno depositada sobre um substrato de h-BN.

\subsection{O Nitreto de Boro Hexagonal - h-BN}

$\mathrm{O}$ nitreto de boro hexagonal - h-BN - ilustrado na figura 6.2, possui uma estrutura em camadas similar ao grafite e devido a sua cor branca, é comumente chamado de grafite branco, como podemos observar na figura 6.2(c).
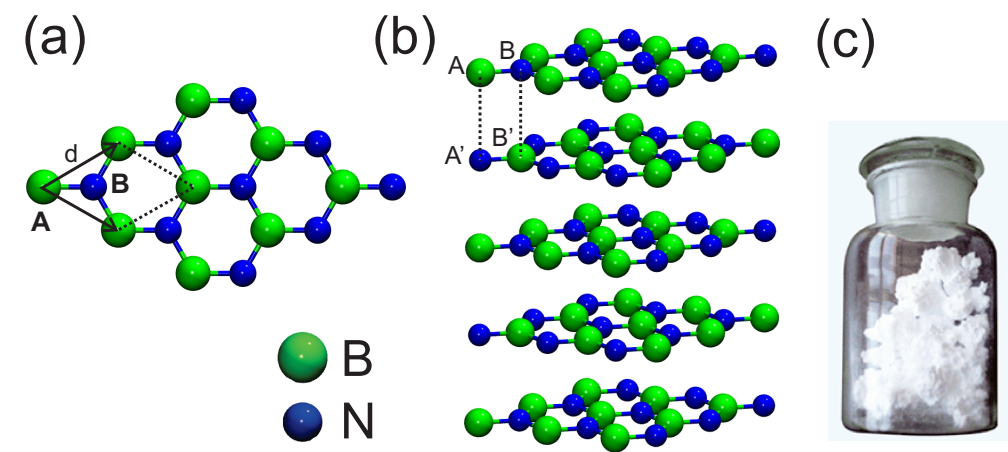

Figura 6.2: (a) Geometria de uma única camada de h-BN, mostrando os átomos de boro e nitrogêncio ocupando as subredes A e B da estrutura hexagonal. (b) Empilhamento $A A^{\prime}$ das monocamadas de h-BN (estrutura encontrada na natureza). (c) Nitreto de Boro Hexagonal vendido comercialmente (conhecido como grafite braco).

$\mathrm{O}$ cristal de h-BN, é composto por monocamadas de h-BN, onde cada uma é composta de átomos de boro e nitrogênio, localizados em uma rede hexagonal como a do grafeno, conforme ilustrado na figura 6.2(a,b). A interação entre as camadas de h-BN é fraca, como no

\footnotetext{
${ }^{1} \mathrm{O}$ empilhamento $A A^{\prime}$ é tal que os átomos de boro de uma camada estão situados acima e/ou abaixo dos átomos de nitrogênio das camadas vizinhas.

${ }^{2}$ Interação de van der Waals
} 
grafite, ou seja, interação de van der Waals. Entretanto, devido a um ganho de energia adicional de Madelung o empilhamento do tipo $A A^{\prime}$ mostrado na figura 6.2(b) é favorecido. Da mesma forma que o grafeno pode ser obtido a partir da exfoliação do grafite, era esperado que uma única camada de nitreto de boro hexagonal também pudesse ser isolada. De fato, essa observação foi primeiramente reportada experimentalmente em 2005 usando clivagem micromecânica [109]. Recentemente, alguns grupos reportaram processos de fabricação de uma única ou algumas camadas de h-BN utilizando outros processos equivalentes para o grafeno, como CVD [107, 110-114].

Sabe-se da literatura, que a estrutura eletrônica de sistemas baseados em grafeno, são fortemente sensíveis à qualquer variação da sua geometria, como por exemplo stress. Dessa maneira, como iremos utilizar o nitreto de boro hexagonal como um substrato para as bicamadas de grafeno e como sabemos que existe uma pequena incompatibilidade entre os parâmetros de rede do grafeno e do h-BN, primeiramente iremos estudar qual o efeito de utilizarmos o parâmetro de rede do grafeno para a célula do h-BN ${ }^{3}$. Na figura 6.3, temos a estrutura de bandas do nitreto de boro hexagonal utilizando a estrutura convergida para o parâmetro de rede do (a) h-BN e (b) do grafeno. Como podemos notar, não existe nenhuma mudança significativa entre as duas estruturas de bandas, indicando que a utilização do parâmetro de rede do grafeno para o sistema h-BN não irá introduzir efeitos espúrios devido ao stress introduzido no h-BN.

Seguindo com a descrição da estrutura eletrônica do h-BN, sabe-se que quando este sistema é utilizado como substrato ou dielétrico, ele estará sujeito a interações com campos elétricos, e um problema que surge é que a voltagem de quebra do dielétrico do h-BN é está entre 0.3 e $0.7 \mathrm{~V} / \mathrm{nm}$. Porém esta voltagem são para substratos de alguns nanometros de espessura. Assim, primeiramente iremos estudar qual a influência de campos elétricos perpendiculares quando consideramos sistemas com apenas algumas camadas de h-BN.

Para simular os filmes finos de h-BN na presença de um campo elétrico, utilizamos cálculos de primeiros princípios baseados na teoria do funcional da densidade, como implementado no código SIESTA. Para o termo de troca e correlação, utilizamos a aproximação local da densidade, LDA. Todas as geometrias foram relaxadas até que as forças ficassem menores que $0.02 \mathrm{eV} / \AA$ A. Consideramos filmes de 1 até 18 camadas de h-BN no empilhamento $A A^{\prime}$.

\footnotetext{
${ }^{3}$ Decidimos estressar o substrato, no lugar do grafeno, pois sabe-se que o grafeno é sensível a qualquer modificação, principalmente stress.
} 


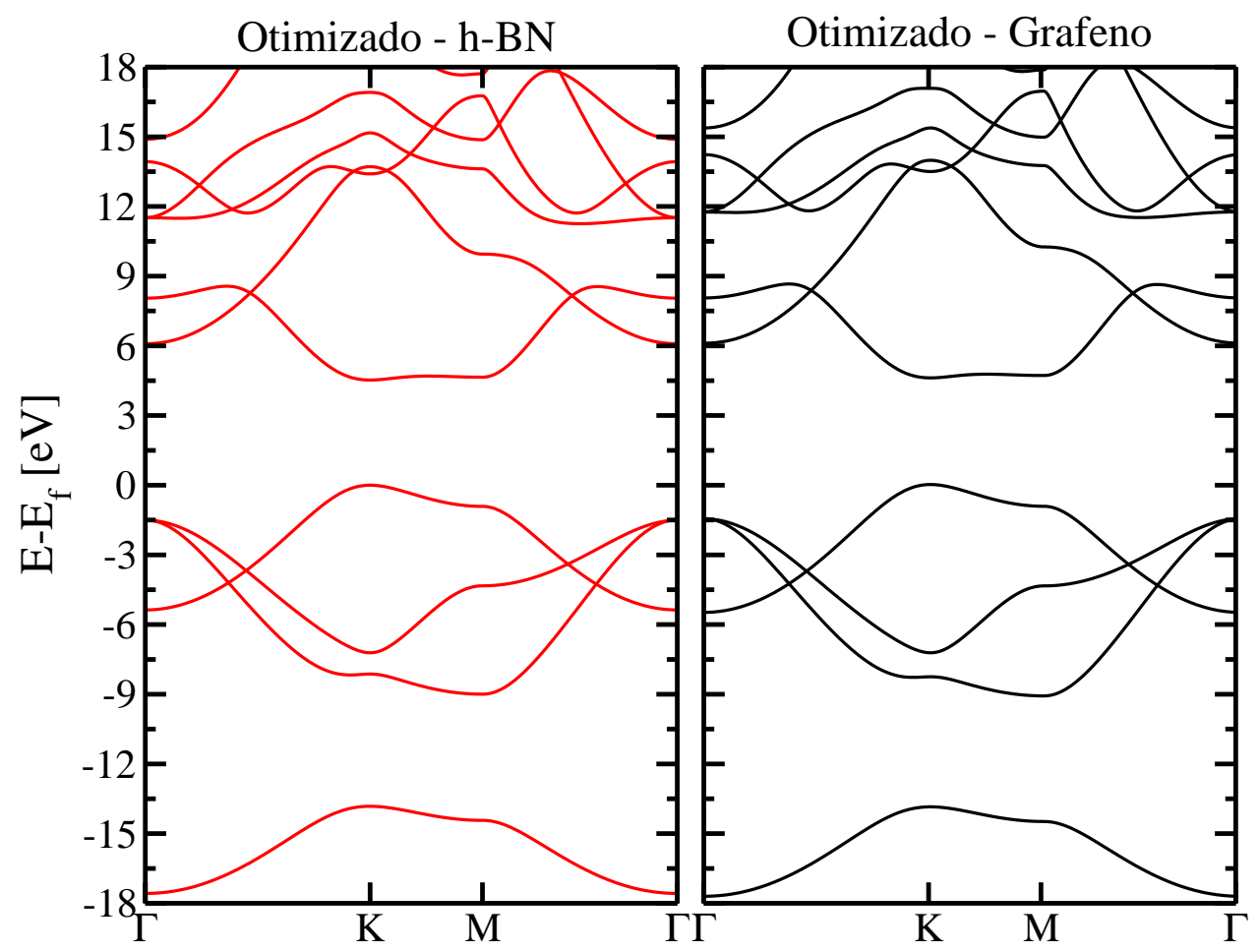

Figura 6.3: Estrutura de bandas para o Nitreto de Boro Hexagonal utilizando o parâmetro de rede do (a) h-BN e (b) do grafeno.

A figura 6.4, mostra as bandas de energia para um sistema com 2 e 5 camadas de nitreto de boro hexagonal, na ausência (a1,b1) e presença $(\mathrm{a} 2, \mathrm{~b} 2)$ de campo elétrico externo aplicado de $2 \mathrm{~V} / \mathrm{nm}$, respectivamente. Sem a presença de um campo, o filme de h-BN é um isolante com um gap de energia de $4.27 \mathrm{eV}$ para duas camadas e $4.10 \mathrm{eV}$ para cinco camadas. Aplicando um campo elétrico externo a esses sistemas de $2 \mathrm{~V} / \mathrm{nm}$ a estrutura eletrônica é fortemente modulada. Ambas as bandas de condução e valência são desdobradas em duas e cinco bandas, para o caso de duas e cinco camadas de h-BN, respectivamente. Esse desdobramento ocorre devido à quebra de simetria ao longo do eixo normal às camadas. Devido ao caráter isolante do h-BN, este sistema não blinda de maneira efetiva o campo elétrico aplicado, gerando um gradiente de potencial entre as camadas. Devido a isso, ocorre um desdobramento das bandas como observado na figura 6.4(a2,b2). Ao aumentarmos a intensidade do campo elétrico, as bandas de condução e valência aproximam-se cada vez mais uma das outras, até que para um campo de $39 \mathrm{~V} / \mathrm{nm}$ para duas camadas de h-BN e $8 \mathrm{~V} / \mathrm{nm}$ para cinco camadas de h-BN, este sistema que é um isolante começa a conduzir. Esta voltagem é conhecida como voltagem de quebra do dielétrico. Na referência [115], este comportamento já foi verificado, onde é mostrado que essa transição isolante-metal do h-BN através da aplicação de um campo elétrico externo, é forte- 

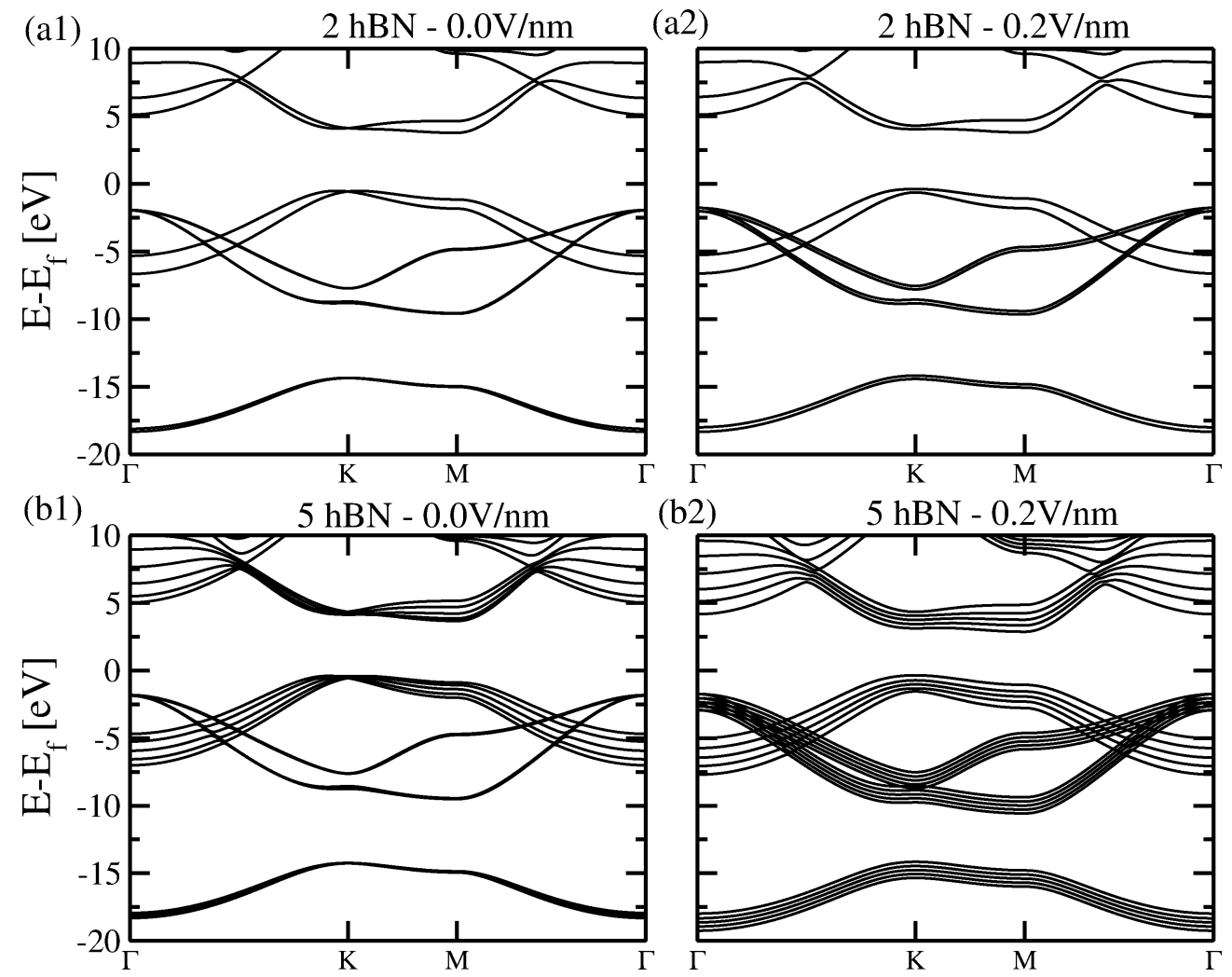

Figura 6.4: Estrutura de bandas para o Nitreto de Boro Hexagonal para 2 h-BN - (a1) sem campo e (a2) com campo de $2 \mathrm{~V} / \mathrm{nm}$, e $5 \mathrm{~h}$-BN - (b1) sem campo e (b2) com campo de $2 \mathrm{~V} / \mathrm{nm}$.

mente dependente da quantidade de camadas de h-BN, onde quanto mais camadas menor é a intensidade do campo necessário para ocorrer a quebra do dielétrico, tendendo ao valor obtido experimentalmente para um sistemas com várias camadas de h-BN que é entre 0.5 e $0.7 \mathrm{~V} / \mathrm{nm}$. Algo importante a notar é que quanto menos camadas de h-BN, maior será o intervalo de voltagens que podem ser aplicadas perpendicularmente ao dielétrico sem ocorrer a quebra desse material. Agora um outro ponto importante, é como a constante dielétrica desse material se comporta em função do campo número de camadas. Sabe-se da literatura que o cristal de h-BN possui uma constante dielétrica entre $3-4$. Dessa maneira, iremos estudar qual a dependência da constante dielétrica do sistema h-BN em função do número de camadas e do campo elétrico.

No cálculo da constante dielétrica do h-BN, devemos estar cientes que uma relação mais geral da resposta dielétrica do sistema sistema não-homogêneo deve incluir efeitos não locais da seguinte forma:

$$
E_{\text {int }}(\vec{r})=\int d \vec{r}^{\prime} \varepsilon^{-1}\left(\vec{r}, \vec{r}^{\prime}\right) E_{\text {ext }}\left(\vec{r}^{\prime}\right)
$$


As correções de campos locais na equação (6.1) implicam que mesmo para um campo externo constante, o campo interno $E_{\text {int }}$ depende de $\vec{r}$. Como é numericamente muito difícil extrair a informação completa sobre $\varepsilon^{-1}\left(\vec{r}, \vec{r}^{\prime}\right)$ a partir de uma abordagem de campos finitos, iremos utilizar uma descrição macroscópica, através de uma constante dielétrica $\varepsilon$, que é independente de $\vec{r}$ e $\vec{r}$ dentro da camada. Para isso, seguiremos uma formulação bem simples da eletrodinâmica macroscópica. Podemos definir um campo espacial médio interno $E_{\text {int }}^{A u c}$ como:

$$
E_{\text {int }}^{a v}=E_{\text {ext }}-4 \pi P\left(E_{\text {ext }}\right),
$$

onde $P$ é o momento de dipolo, por unidade de volume, induzido pelo campo externo homogêneo, $E_{\text {ext }}$, e $P$ é dado por:

$$
P=\frac{p_{z}}{A_{u c} d},
$$

onde $A_{u c}$ é a área da célula unitária do sistema, $d$ é a distância entre as camadas inferior e superior do sistema e $p_{z}$ é o momento de dipolo induzido por célula unitária. Para o cálculo de $p_{z}$, vamos considerar uma média da densidade de carga induzida no plano $x-y, \bar{n}(z)$, utilizada para o cálculo do momento de dipolo induzido por célula unitária, $p_{z}$,

$$
p_{z}=-|e| A_{u c} \int d z z \bar{n}(z),
$$

onde $e$ denota a carga elementar e $A_{u c}$ a área da célula unitária no plano $x-y$.

Na figura 6.5(a), mostramos a média planar das densidades eletrônicas induzidas para um sistema com cinco camadas de h-BN. Devido a baixa blindagem que as camadas de h-BN exercem sobre o campo elétrico, a densidade eletrônica induzida na superfície varia muito pouco com respeito à parte interna do sistema, de maneira contrária ao que acontece com as multicamadas de grafeno [116], onde a maior parte da carga induzida fica localizada na superfície da camada.

Seguindo, podemos tomar uma definição diferencial do campo elétrico interno:

$$
d E_{\text {int }}^{a v}\left(E_{\text {ext }}\right)=\varepsilon^{-1}\left(E_{\text {ext }}\right) \cdot d E_{\text {ext }} .
$$

Definindo uma constante dielétrica média $\bar{\varepsilon}$ através da integração da equação 6.5 ,

$$
\frac{1}{E_{\text {ext }}} \int_{0}^{E_{\text {ext }}} d E_{\text {ext }}^{\prime} \cdot \varepsilon^{-1}\left(E_{\text {ext }}^{\prime}\right)=E_{\text {int }}^{a v}\left(E_{\text {ext }}\right) / E_{\text {ext }} \equiv \bar{\varepsilon}^{-1}\left(E_{\text {ext }}\right) .
$$



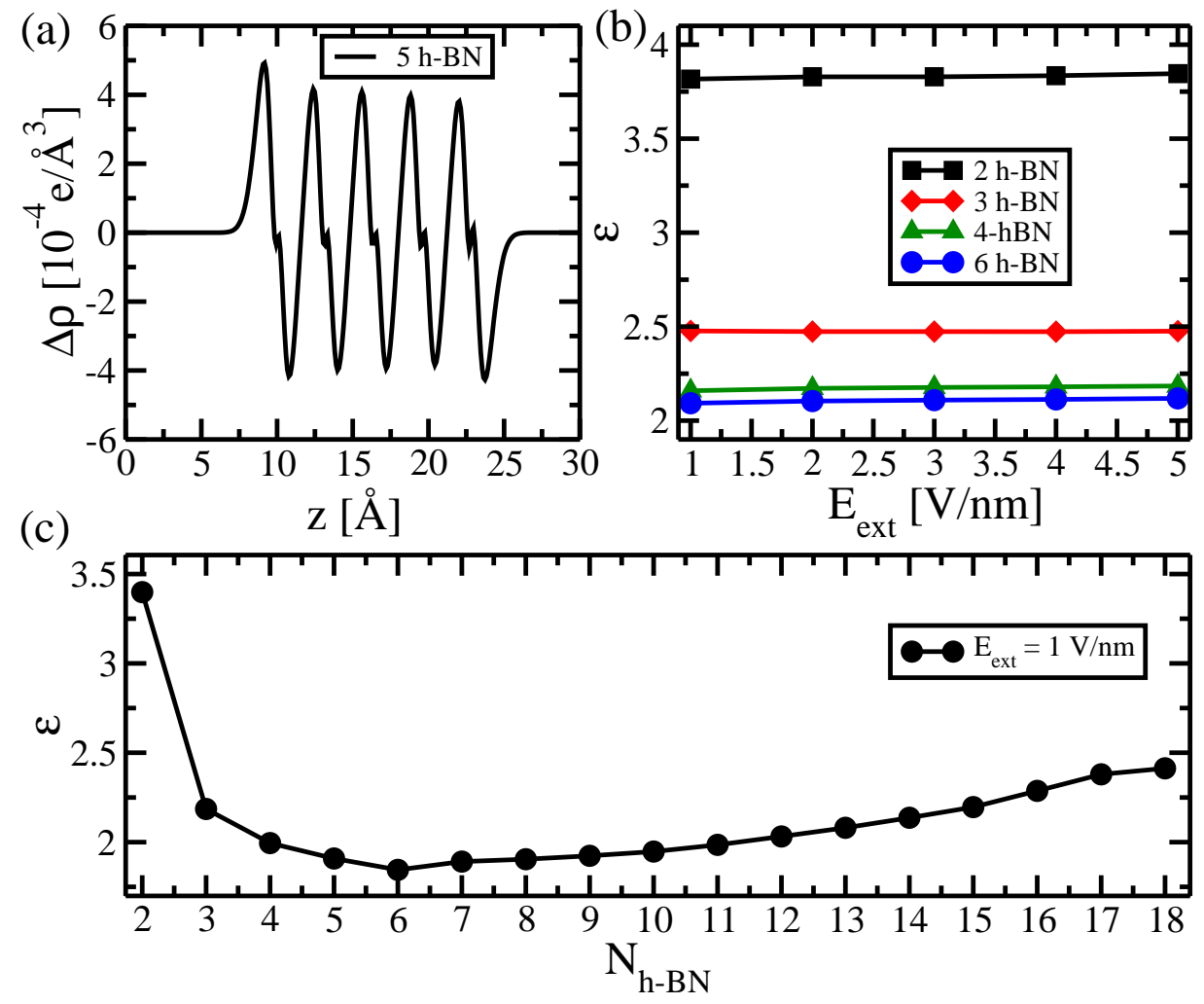

Figura 6.5: (a) Média planar da densidade eletrônica induzida para cinco camadas de h-BN na presença de um campo de $1 \mathrm{~V} / \mathrm{nm}$. (b) Constante dielétrica média em função do campo elétrico externo para 2, 3, 4 e 6 camadas de h-BN. (c) Constante dielétrica média em função do número de camadas de h-BN para um campo elétrico de $1 \mathrm{~V} / \mathrm{nm}$.

Usando a equação 6.2, temos:

$$
\bar{\varepsilon}^{-1}\left(E_{\text {ext }}\right)=1-4 \pi \frac{P\left(E_{\text {ext }}\right)}{E_{\text {ext }}} .
$$

Apesar da ligação fraca entre a função resposta dielétrica total não-local do sistema, $\bar{\varepsilon}$ fornece uma informação exata sobre o momento de dipolo induzido por área superficial do sistema.

Como podemos observar da figura 6.5(b), $\bar{\varepsilon}$ possui um valor constante em função do campo elétrico externo. Este comportamento ocorre devido à baixa blindagem do sistema em relação ao campo elétrico externo. As cargas induzidas devido ao campo elétrico externo se distribuem em todo o sistema, não ficando somente localizadas na superfície do sistema. Na figura 6.5(c), mostramos o valor de $\bar{\varepsilon}$ em função do número de camadas de h-BN. Primeiramente, notamos que para um sistema com poucas camadas, o valor de $\bar{\varepsilon}$ é da ordem de 3.4, comparável com os valores do cristal de h-BN, porém cai rapidamente aumentando-se o número de camadas de 
h-BN.

Outro ponto, é que ao aumentarmos o número de camadas a partir de seis camadas, este valor torna a aumentar, como era de se esperar, pois este valor deve voltar a tender para o cristal de h-BN. De posse dessas informações e do comportamento da voltagem de quebra do dielétrico que observamos anteriormente, podemos concluir que para a utilização do nitreto de boro hexagonal como dielétrico, devemos ter poucas camadas, para preservar as propriedades dielétricas do h-BN. Além disso, com poucas camadas, o intervalo de voltagens que podem ser aplicadas ao dispositivo sem deteriorar suas propriedades é maior.

\subsection{Bicamadas de Grafeno Sobre Substrato de Nitreto de Boro Hexagonal - BG+h-BN}

Nesta seção iremos estudar de maneira detalhada, qual a influência do substrato de nitreto de boro hexagonal nas propriedades eletrônicas das bicamadas de grafeno.

(I)
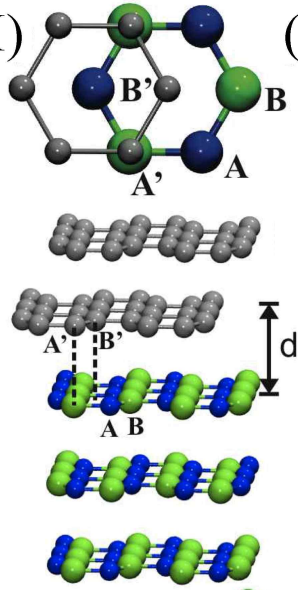

(II)
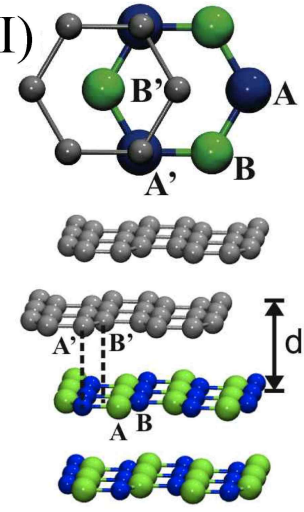

Boro

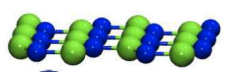

Nitrogênio
(III)
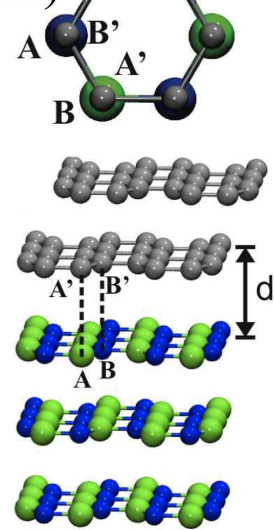

Carbono

Figura 6.6: Representação estrutural dos possíveis empilhamentos da bicamada de grafeno em relação ao substrato de h-BN.

Para simular a bicamada de grafeno sobre filmes finos de h-BN na presença de um campo elétrico externo, iniciamos os cálculos para o h-BN com o empilhamento do bulk, $A A^{\prime}$. Na figura 6.6, apresentamos as três possibilidades de empilhamento da bicamada de grafeno sobre o h-BN $\left(A A^{\prime}\right)$. Variando a distância $d$ entre a camada superior de h-BN com a camada inferior da bicamada de grafeno, obtemos a conformação $I$ como a mais estável, com uma distância de equilíbrio de $3.18 \AA$. Na figura 6.7(b) mostramos as curvas de energia potencial para os 
sistemas $(I, I I, I I I)$ da figura 6.6 e também para o sistema consistindo de somente uma camada de grafeno, figura 6.7(a). Como era de se esperar, a bicamada de grafeno possui a mesma configuração de mais baixa energia que o grafeno.
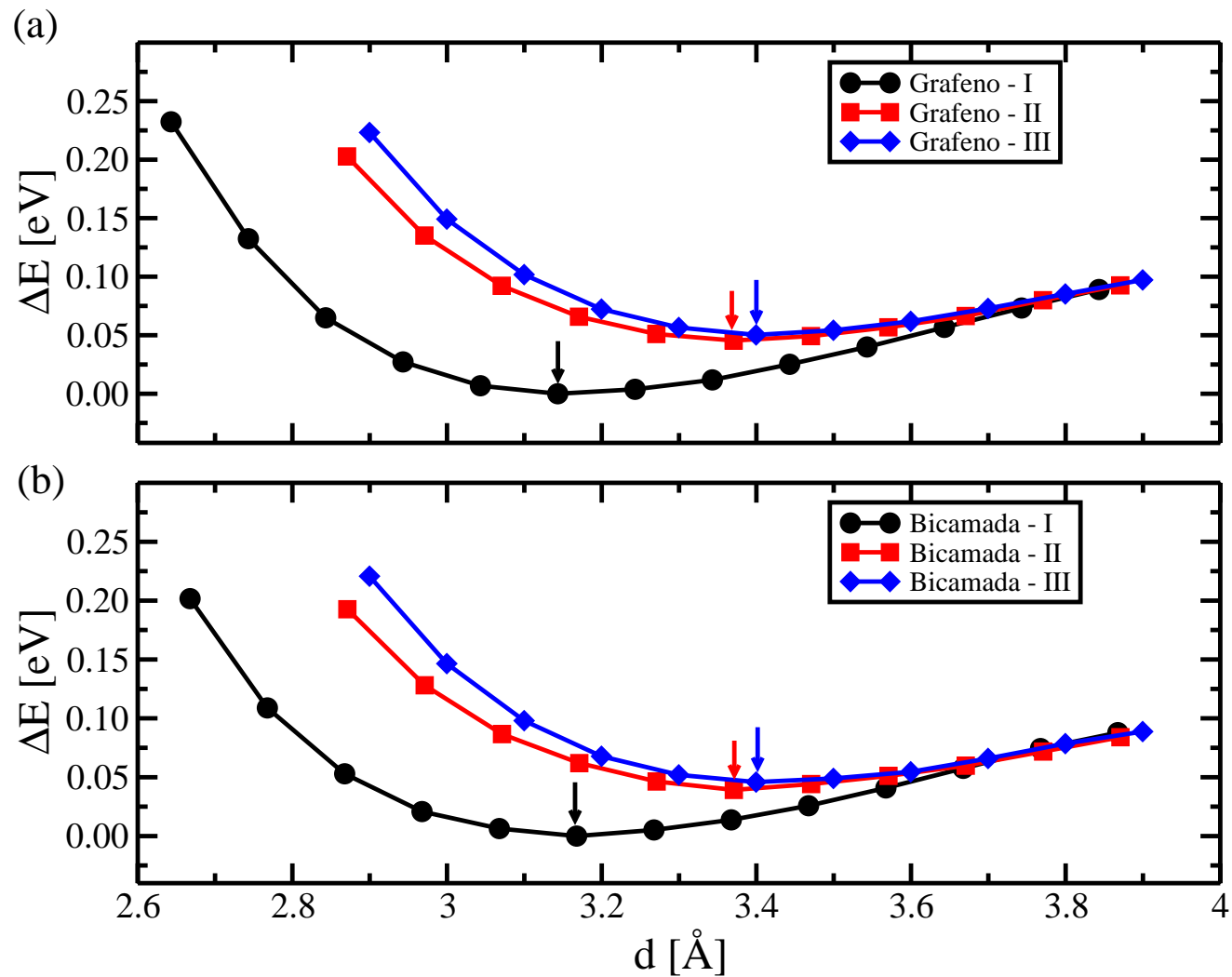

Figura 6.7: Energia total em função da distância $d$ de separação entre a camada superior de h-BN com a camada inferior para (a) grafeno e (b) bicamada de grafeno, nos empilhamentos mostrados na figura 6.6. Os valores das energias são dados em relação à configuração com menor energia para cada sistema. Essas curvas foram construídas utilizando a aproximação LDA para o termo de troca e correlação.

Nesses cálculos utilizamos a aproximação local da densidade (LDA) na forma de CeperleyAlder (CA) [66] para o funcional de troca e correlação. Entretanto, como estamos tratando com sistemas fracamente interagentes, onde forças de dispersão (como interação de longo alcance como van der Waals) exercem um papel fundamental na energética do sistema, realizamos também cálculos com uma forma semi-empírica para um funcional do tipo GGA como proposto por Grimme [67], que leva em conta estes tipos de interações. Entretanto, mesmo usando este tipo de funcional, a configuração $I$ continua sendo energeticamente favorável. Como não estamos interessados no comportamento assintótico da energia de interação entre as camadas, todos os cálculos foram realizados utilizando a aproximação LDA para o termo $E_{x c}$.

Na configuração energética mais estável para o sistema bicamada de grafeno sobre substrato 

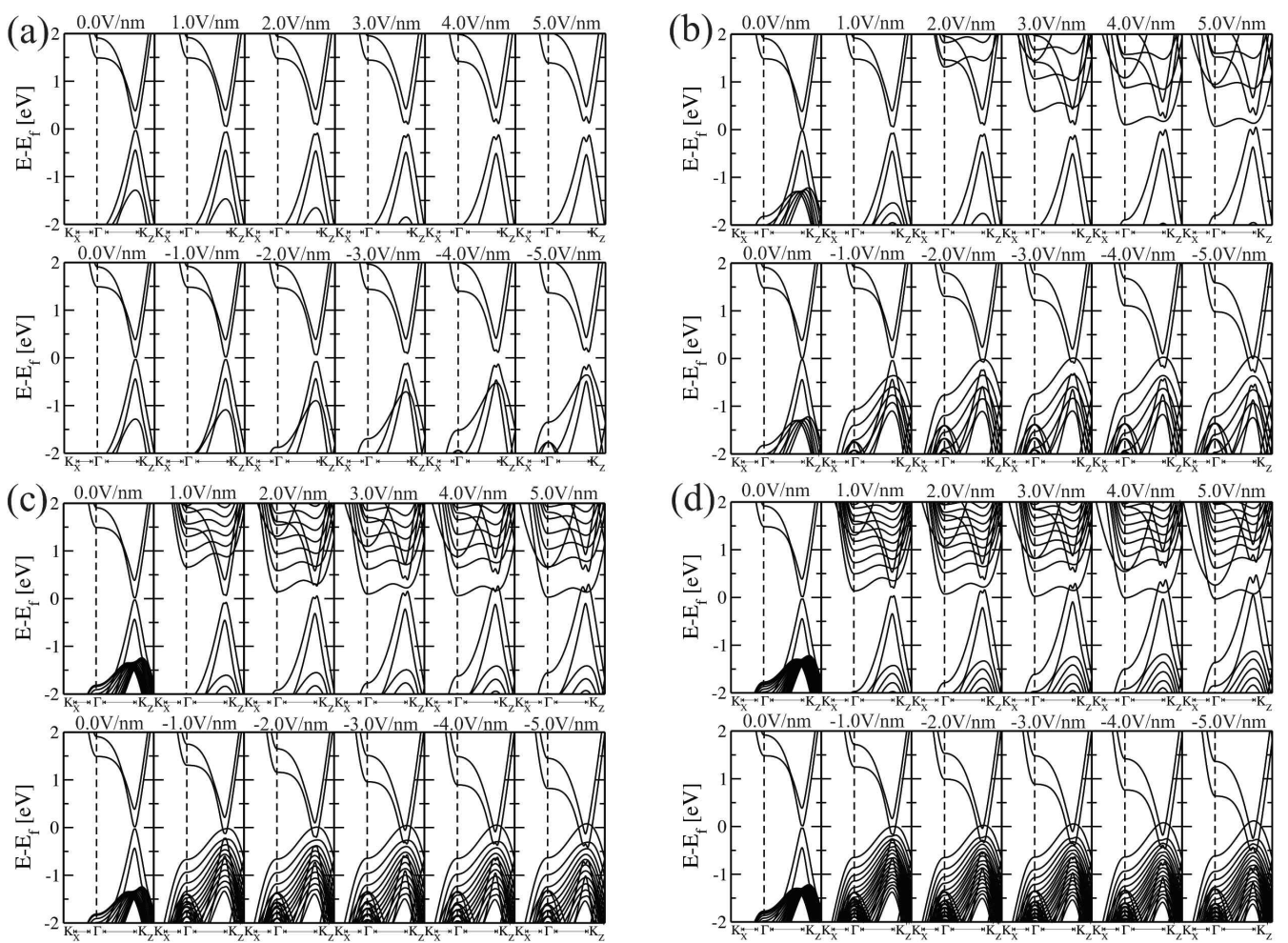

Figura 6.8: Estrutura de bandas para as bicamadas de grafeno sobre h-BN(AA'), em função do número de camadas de h-BN para: (a) 1 h-BN; (b) 5 h-BN; (c) 12 h-BN e (d) 18 h-BN, na presença de um campo elétrico perpendicular, variando de $+1 \mathrm{~V} / \mathrm{nm}$ a $+5 \mathrm{~V} / \mathrm{nm}$ painéis superiores e $-1.0 \mathrm{~V} / \mathrm{nm}$ a $-5.0 \mathrm{~V} / \mathrm{nm}$ painéis inferiores. O nível de Fermi do sistema está no zero.

de nitreto de boro $(B G+h-B N)$, consideramos o efeito de um campo elétrico externo aplicado. A evolução das estruturas de bandas em função de ambos, o campo elétrico e número de camadas de h-BN, está apresentado na figura 6.8. Os painéis superiores e inferiores correspondem a campos elétricos positivos e negativos, respectivamente ${ }^{4}$. O primeiro ponto interessante é que para a bicamada de grafeno adsorvida no topo do substrato de h-BN, existe a abertura de gap de energia, $\Delta g$, de aproximadamente $45 \mathrm{meV}$, mesmo na ausência de um campo elétrico. Isso ocorre devido a uma quebra de simetria no sistema. Quando um campo elétrico é aplicado ao sistema, ocorre um aumento do gap $\Delta g$ e $\Delta K$ (gap no ponto de alta simetria $K$ ), quando o número de camadas de h-BN é aumentada. Uma visão esquemática dos gaps $\Delta g$ e $\Delta K$ na bicamada de grafeno pode ser visto na figura 6.9(b), painel inferior.

A variação dos gaps na bicamada de grafeno em função de ambos o campo elétrico e o número de camadas de h-BN são apresentados na figura $6.9(\mathrm{c})^{5}$. Podemos observar que os

\footnotetext{
${ }^{4}$ Campos elétricos positivos e negativos significam em qual direção o campo elétrico é aplicado, ao longo de $\pm \hat{z}$

${ }^{5}$ Vale mencionar que ambas as aproximações LDA e GGA subestimam o valor do gap de energia. Conseqüen-
} 


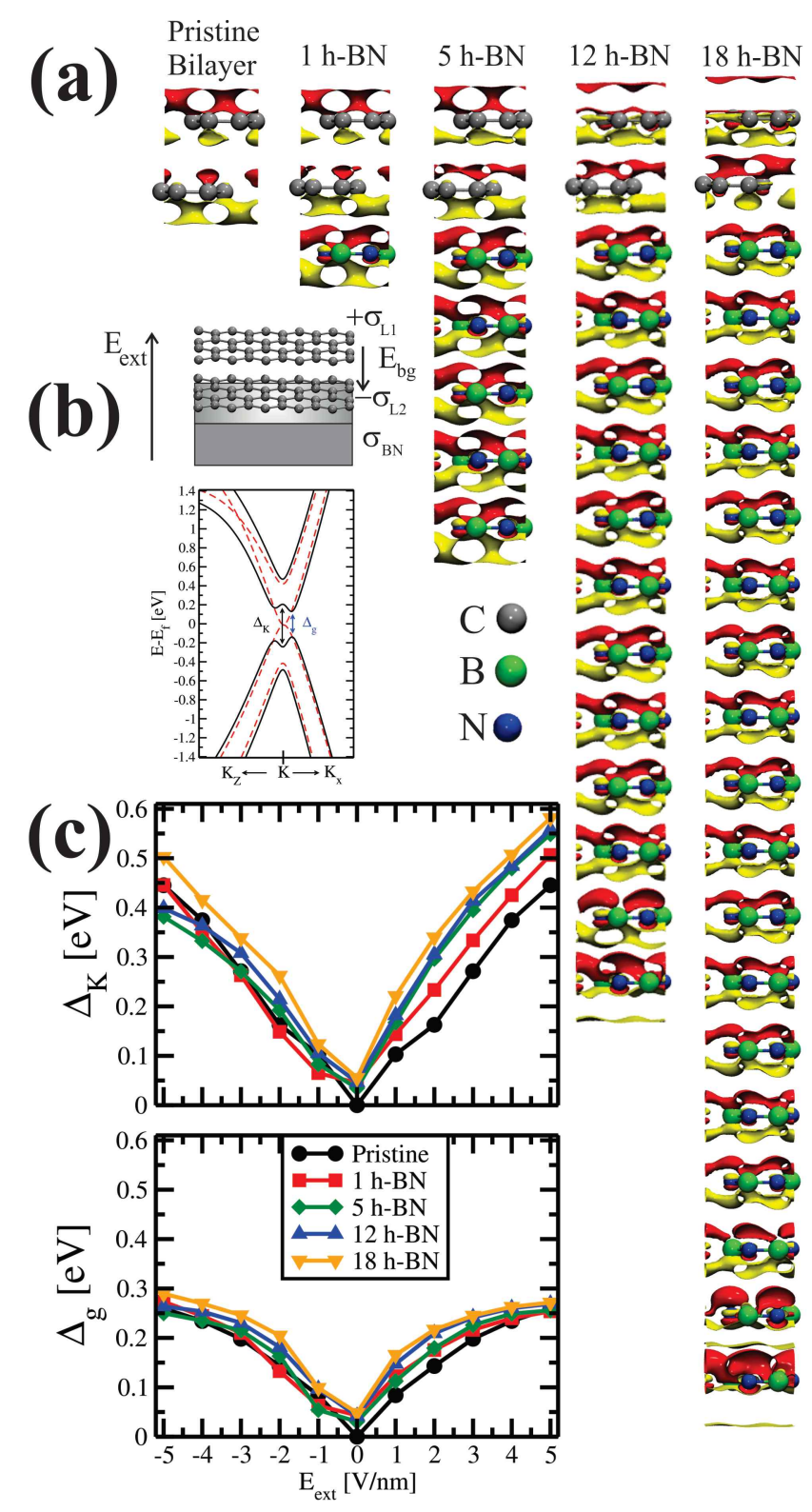

Figura 6.9: (a) Diferença entre as densidades de carga na presença de um campo elétrico de $+2 \mathrm{~V} / \mathrm{nm}$ e sem campo, $\Delta \rho=\rho_{E}-\rho_{0}$. Vermelho indica $\Delta \rho$ positivo e amarelo indica $\Delta \rho$ negativo. $\mathrm{O}$ valor da isosuperfície utilizada foi de $1 \times 10^{-4} e / b o h r^{3}$. (b) Visão esquemática dos gaps de energia $\Delta K \mathrm{e} \Delta g$, na bicamada de grafeno. (c) Gaps de energia nas bicamadas de grafeno em função do número de camadas de h-BN e do campo elétrico aplicado.

gaps na bicamada de grafeno aumentam, quando comparados com o sistema sem o substrato de h-BN. Para entender porque isso está ocorrendo, podemos olhar primeiramente para o gap no ponto $K$ para a bicamada de grafeno pristina. Este gap pode ser escrito em uma forma fechada como

$$
\Delta K=\left|d E_{\text {eff }} e\right|
$$

temente, os valores dos gaps de energia apresentados representam um limite inferior. 
onde $d$ é a distância entre as camadas, e é a carga elementar do elétron e $E_{\text {eff }}$ é o campo efetivo na bicamada de grafeno [117]. Na presença de um campo elétrico externo aplicado perpendicularmente ao sistema, ocorre uma transferência de carga entre as camadas da BG. As cargas ficam localizadas acima e abaixo da bicamada, representadas na figura 6.9(b) por $\sigma_{L 1}$ e $\sigma_{L 2}$. Estas cargas geram um campo de polarização, $E_{B G}$, que é contrário ao campo externo, $E_{\text {ext }}$. Assim, o campo efetivo que muda a energia do sítio, e por conseqüência quebra a simetria entre as duas camadas abrindo o gap, é dado por:

$$
E_{e f f}=E_{e x t}-E_{B G}
$$

Para o sistema $B G+h-B N$, quando aplicamos um campo elétrico externo, ocorre a transferência de carga entre as camadas da bicamada de grafeno, mas também ocorre uma transferência de carga da bicamada de grafeno para o substrato de h-BN. Essa redistribuição de carga no sistema inteiro $(B G+h-B N)$, reduz o campo de polarização, $E_{B G}$, e conseqüentemente ocorre um aumento do campo efetivo, $E_{\text {eff }}$, que leva um aumento dos gaps de energia representados na figura 6.9(c). Dessa maneira, o gap de energia na bicamada de grafeno, depende tanto da intensidade do campo elétrico externo, como do número de camadas de h-BN.

A figura 6.9(a) mostra a diferença entre as densidades de cargas $(\Delta \rho)$, para os sistemas com um campo de $+2.0 \mathrm{~V} / \mathrm{nm}$ e sem campo. Podemos claramente notar que aumentando o número de camadas de h-BN, ocorre um aumento da densidade de carga dentro da bicamada de grafeno, que é refletido pelo aumento no campo efetivo desse sistema, levando a um aumento do gap de energia. Além disso, como as camadas de nitreto de boro hexagonal não blindam efetivamente o campo externo, o sistema fica sujeito a um gradiente de potencial entre as camadas e conseqüentemente ocorre um deslocamento dos níveis de energia do sistema.

Entretanto, na presença de um campo elétrico externo aplicado, os níveis de energia que são deslocados para o nível de Fermi do sistema vem do substrato de h-BN, ocorrendo uma abertura de um canal de transporte proveniente do dielétrico, tornando o sistema metálico. A definição de voltagem de quebra de um isolante é a voltagem mínima que leva uma porção do isolante virar um condutor. Estimamos essa voltagem em função do número de camadas de h-BN.

Começando para o caso de uma única camada de h-BN, notamos que aplicando um campo 
de até $5 \mathrm{~V} / \mathrm{nm}$, o potencial químico do sistema continua no meio do gap do sistema, não ocorrendo nenhuma inserção de níveis do substrato, mantendo então as características semicondutoras do sistema. Quando aumentamos o número de camadas de nitreto de boro, mais níveis do substrato são adicionados perto da banda de valência do sistema, ao redor de $-1.2 \mathrm{eV}$, como podemos observar na figura 6.8(b)-(d)(sem campo elétrico). Quando um campo elétrico negativo é aplicado ao sistema, os estados ocupados do nitreto de boro (inicialmente em $-1.2 \mathrm{eV}$ ) são levantados até a energia de Fermi do sistema e como podemos observar, quando aumentamos o número de camadas de h-BN, a intensidade do campo necessário para colocar esses estados no nível de Fermi fica cada vez menor, 6.8(painéis inferiores). Por exemplo, para 5 camadas, o campo elétrico necessário é de $\approx-1.5 \mathrm{~V} / \mathrm{nm}$ enquanto que para 12 e 18 camadas, é de aproximadamente $-0.6 \mathrm{~V} / \mathrm{nm}$. Como podemos observar na figura 6.8(painéis superiores), o mesmo comportamento é observado quando aplicamos um campo elétrico positivo. Nesse caso, os níveis do h-BN inicialmente localizados em $+3.5 \mathrm{eV}$ (fora da escala do gráfico), são gradualmente abaixados para a energia de Fermi. Por exemplo, para 5 camadas de h-BN, o campo elétrico necessário para colocar um estado do $B N$ no nível de Fermi foi de $+4.0 \mathrm{~V} / \mathrm{nm}$, enquanto que para 18 camadas, o campo foi de $\approx+1.0 \mathrm{~V} / \mathrm{nm}$. Assim, a voltagem de quebra do dielétrico nesses sistemas, não só depende do número de camadas de h-BN, como também da direção do campo elétrico aplicado. Comparando esses resultados para o sistema $B G+h-B N$ com o sistema de h-BN pristino, observamos que a voltagem de quebra do dielétrico é reduzida. Isso ocorre principalmente devido à transferência de carga entre a bicamada de grafeno e o substrato de h-BN.

Para ilustrar o comportamento dos níveis de energia dos sistema, analisamos a densidade de estados projetada (PDOS) para o sistema com 5 camadas de h-BN. Na figura 6.10(a) mostramos a PDOS, para cada camada sem a presença de campo elétrico. Os níveis associados com os estados ocupados no h-BN aparecem ao redor de $-1.2 \mathrm{eV}$ abaixo do nível de Fermi (definido como zero) e os níveis associados com estados desocupados do BN estão ao redor de $+3.5 \mathrm{eV}$ (fora da escala da figura). Na figura 6.10(b,c) apresentamos a PDOS para cada camada do sistema, decomposta nos átomos de boro e nitrogênio, na presença de um campo elétrico aplicado. Considerando um campo positivo $(+4.0 \mathrm{~V} / \mathrm{nm})$ [Figura 6.10(b)], verificamos um levantamento dos níveis de energia do boro e nitrogênio com caráter $p$. Além disso, os níveis associados com as camadas mais profundas do h-BN são os mais afetados. Em particular, esses estados 


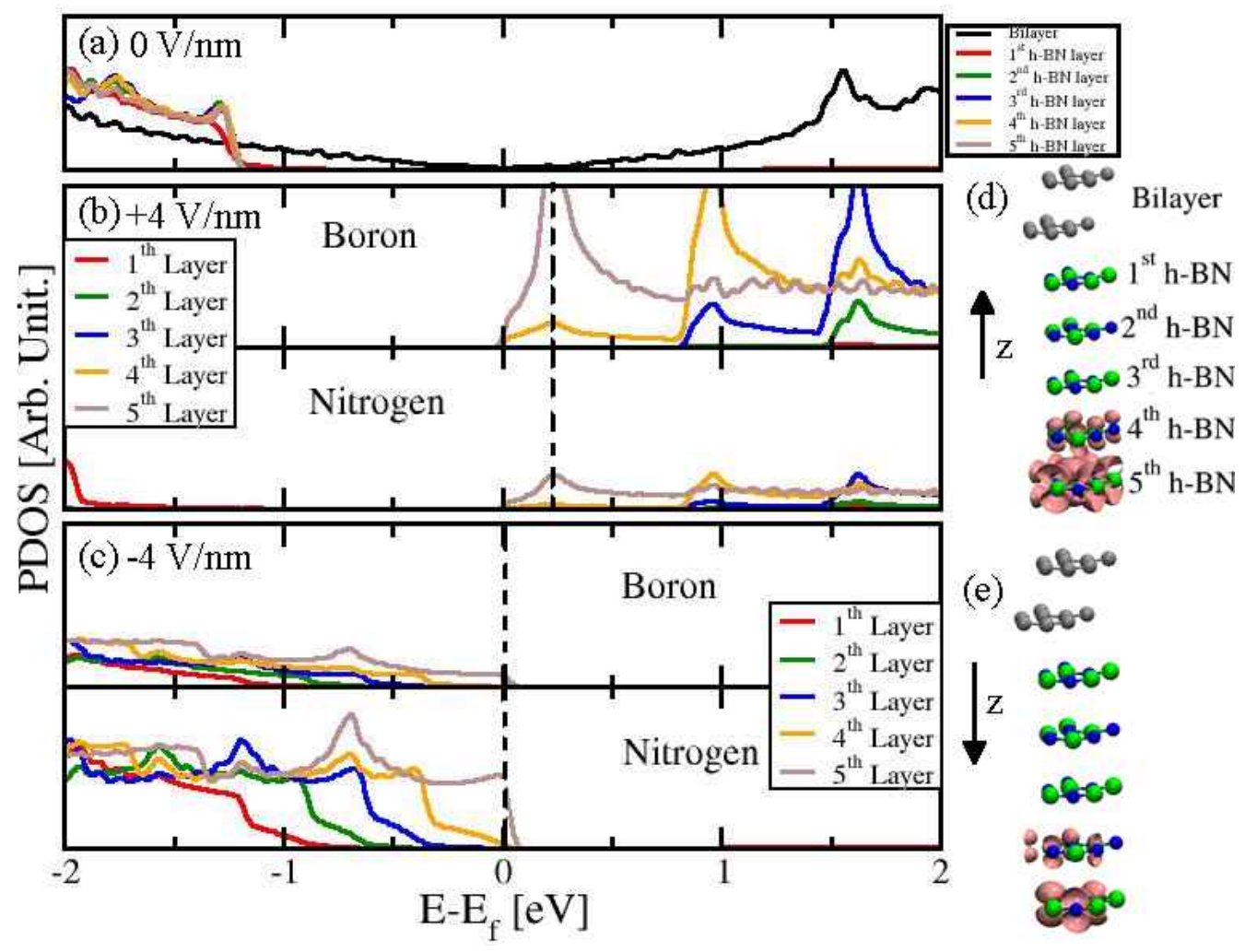

Figura 6.10: Densidade de estados projetada para uma bicamada de grafeno sobre 5 camadas de nitreto de boro hexagonal, (a) sem campo elétrico, (b) com um campo elétrico positivo de $+4.0 \mathrm{~V} / \mathrm{nm}$ e (c) com um campo elétrico negativo de $-4.0 \mathrm{~V} / \mathrm{nm}$. As linhas pontilhadas em (b) e (c) representam a região próxima ao nível de Fermi onde utilizamos para plotar a LDOS (d,e). O nível de Fermi do sistema foi definido como sendo zero na figura.

são levantados até o nível de Fermi, dentro do gap aberto na bicamada de grafeno. Podemos observar isso, através da densidade de estados local (LDOS), para a região pontilhada na figura 6.10(b). O mesmo comportamento é verificado para um campo elétrico negativo, 6.10(c,e). Entretanto, os níveis ocupados do $B N$ estão mais próximos do máximo da banda de valência, quando comparados com os desocupados em relação ao mínimo da banda de condução. Dessa maneira, aplicando-se o mesmo campo elétrico, na direção contrária, $-4.0 \mathrm{~V} / \mathrm{nm}$, níveis até da quarta camada chegam próximos ao nível de Fermi e o nível de quinta camada é deslocado para a banda de condução. A LDOS para essa região em torno do nível de Fermi ilustra esse comportamento, figura 6.10(e). Desse modo, canais de condução através do h-BN são criados. Entretanto, eles estão bem separados da bicamada de grafeno, implicando que estes interagem muito pouco com a BG, que pode ser considerada isolada.

Como mencionamos anteriormente, o empilhamento $A A^{\prime}$ é o inerente ao cristal natural de 
Tabela 6.1: Diferenças de energia total para os seis empilhamentos considerados.

\begin{tabular}{c|c|c}
\hline \hline Empilhamento & LDA (eV) & GGA+vdW (eV) \\
\hline \hline$A B / A B / A B$ & 0.0000 & 0.0000 \\
$A A^{\prime} / A B / A A^{\prime}$ & 0.0082 & 0.0333 \\
$A B / A A^{\prime} / A B$ & 0.0138 & 0.0356 \\
$A A^{\prime} / A A^{\prime} / A A^{\prime}$ & 0.0209 & 0.0634 \\
$A B / A A^{\prime} / A A^{\prime}$ & 0.0204 & 0.0495 \\
$A A^{\prime} / A A^{\prime} / A B$ & 0.0214 & 0.0508 \\
\hline \hline
\end{tabular}

h-BN. Entretanto, durante o processo de exfoliação do material, alguns outros empilhamentos podem ser obtidos [107]. Conseqüentemente, é importante verificarmos se os mesmos resultados obtidos anteriormente são independentes do empilhamento ou se existe alguma dependência do processo de abertura de gap na bicamada de grafeno e da voltagem de quebra do dielétrico para cada tipo de empilhamento do substrato de nitreto de boro hexagonal.

Investigamos tanto a energética desses sistemas como o efeito do campo elétrico aplicado em diversos tipos de empilhamentos do h-BN. A bicamada de grafeno continua na configuração mais estável obtida anteriormente. Estes empilhamentos estão representados na figura 6.11(sem a presença da bicamada de grafeno).

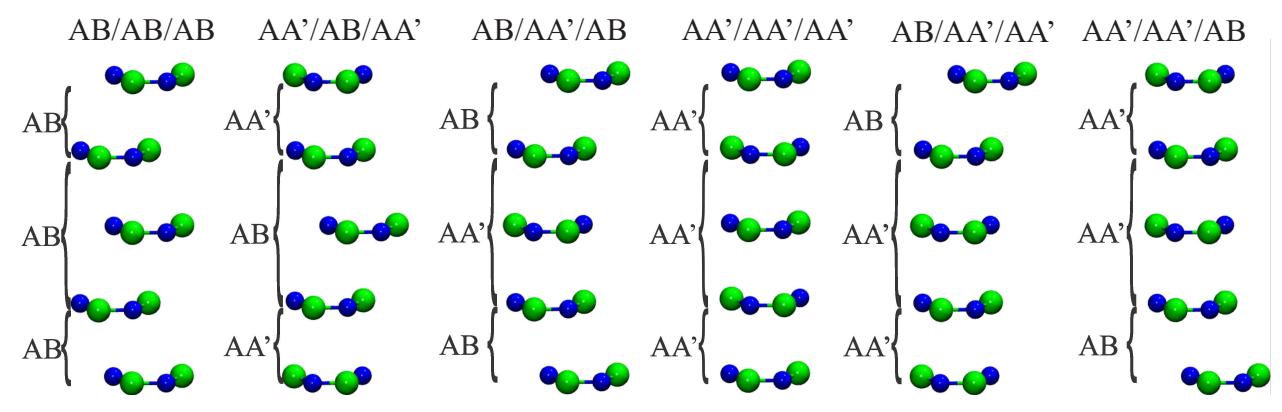

Figura 6.11: Tipos de empilhamentos possíveis para o cristal de h-BN

Do ponto de vista da energética do sistema, mesmo considerando correções para as interações de longo alcance (correções de van der Walls), obtemos que a configuração de mais baixa energia para o h-BN é $\mathrm{AB} / \mathrm{AB} / \mathrm{AB}$, que não é o empilhamento natural do h-BN. Porém, como já observado anteriormente na referência [108], a barreira para transformar do empilhamento $A A^{\prime}$ para o $A B$ é relativamente grande. As diferenças na energia total entre as conformações é pequena, como mostrado na tabela 6.1, confirmando que empilhamentos diferentes do $A A^{\prime}$ podem ocorrer nas camadas de h-BN.

Na figura 6.12, apresentamos as estruturas de bandas para os seis empilhamentos investiga- 
dos para um campo elétrico de $\pm 2 \mathrm{~V} / \mathrm{nm}$.
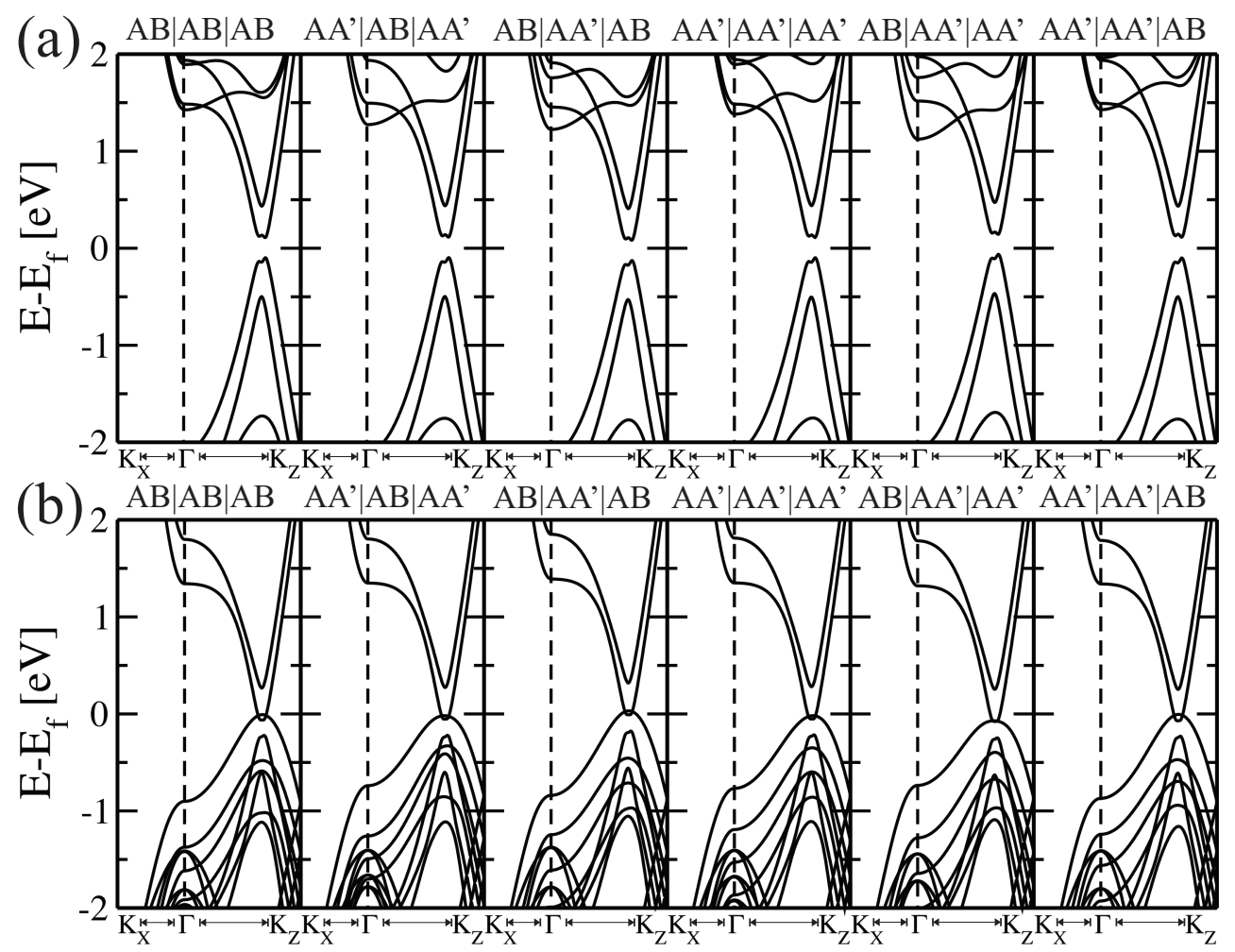

Figura 6.12: Estruturas de bandas para a bicamada de grafeno sobre h-BN. Apresentamos os seis possíveis empilhamentos na presença de um campo elétrico aplicado de (a) $+2 \mathrm{~V} / \mathrm{nm}$ e (b) $-2 \mathrm{~V} / \mathrm{nm}$.

Um ponto importante e notável, é que ao contrário das multicamadas de grafeno, para qualquer tipo de empilhamento do substrato, a forma geral das bandas de energia não muda em função do empilhamento e do campo elétrico aplicado. Na figura 6.13, apresentamos as estruturas de bandas para 5 e 12 camadas de h-BN, no empilhamento $A B / A B / A B$. Como podemos observar, o comportamento é o mesmo que para o $A A^{\prime}$. Dessa maneira, o comportamento da estrutura de bandas e as principais conclusões que obtemos para o empilhamento $A A^{\prime}$ são válidas para quaisquer outros tipos de empilhamentos do h-BN.

\subsection{Conclusões}

Em conclusão, a partir de cálculos de primeiros princípios de estrutura eletrônica baseados em DFT, mostramos que é possível criar um gap de energia controlável na bicamada de grafeno sobre um substrato de nitreto de boro hexagonal. Este gap fica ligeiramente maior quanto maior é o número de camadas de h-BN. Mostramos também que na presença de um campo elétrico, níveis do substrato de h-BN são inseridos no nível de Fermi do sistema, fazendo 


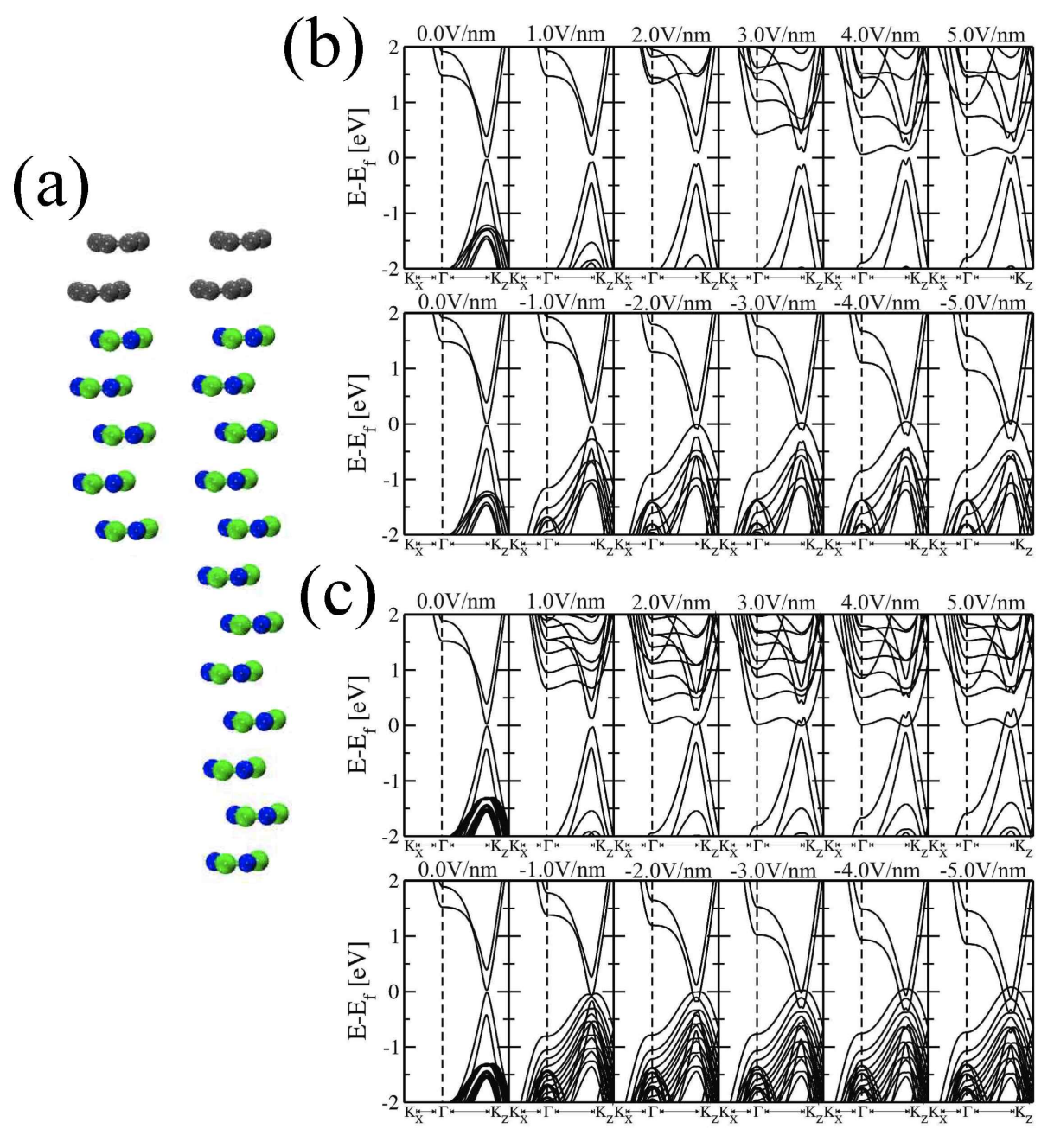

Figura 6.13: (a) Visão esquemática da bicamada de grafeno sobre h-BN no empilhamento $A B / A B / A B$, para 5 e 12 camadas; (b) Estrutura de bandas para os sistemas em (a), em função do campo elétrico aplicado, variando de $\pm 1 \mathrm{~V} / \mathrm{nm}$ até $\pm 5 \mathrm{~V} / \mathrm{nm}$. Como verificado para o empilhamento $A A^{\prime}$, quanto menos camadas presente, maior fica a intensidade do campo elétrico necessário para criar um canal de condução através do substrato.

com que este fique metálico. Além disso, o range de voltagens que podem ser aplicadas no dispositivo depende fortemente do número de camadas de h-BN e da direção do campo elétrico. Finalmente, a voltagem de quebra do dielétrico e a possibilidade da criação do gap na bicamada é independente do tipo de empilhamento do substrato de h-BN. 


\section{Capítulo 7}

\section{Interações entre canal e dielétrico com Cobre(111)}

Na nanoeletrônica, o grafeno pode ser utilizado como um dos componentes básicos de um dispositivo, principalmente na forma de eletrodo ou canal de um nanotransistor. Na maior parte destas aplicações, existe a necessidade da utilização de outro material que suporte o grafeno, criando uma interface entre o grafeno e este outro sistema. Isso influência a resposta global do dispositivo. Desse modo, é fundamental o entendimento da influência deste outro material nas propriedades eletrônicas do grafeno. No capítulo 6, realizamos um estudo das propriedades das bicamadas de grafeno sobre um substrato isolante de h-BN. Entretanto, além do substrato, existe ainda os contatos metálicos que são utilizados como eletrodos em um dispositivo.

Com o advento da produção do grafeno epitaxial em larga escala, principalmente sobre a superfície de cobre(111), por meio de um processo de fabricação relativamente simples, foi possível a obtenção de amostras de grafeno macroscópicas [118]. Facilitando, assim, os estudos das propriedades físicas e químicas deste material. Este avanço também destaca a importância da interação entre o grafeno e o cobre. Na figura 7.1, mostramos um exemplo da produção de grafeno em larga escala (com aproximadamente $1 \mathrm{~m}^{2}$ de área), utilizando técnicas de CVD. Neste trabalho, o grupo da SAMSUNG aplicou este grafeno em telas touch-screen [118].

Do mesmo modo que o grafeno, finas camadas de h-BN podem ser preparadas por exfoliação mecânica [109]. De maneira alternativa à exfoliação, camadas de nitreto de boro hexagonal podem também ser obtidas através de técnicas de CVD sobre metais de transição, como o níquel e o cobre, usando precursores como a borazina $\left(B_{3} N_{3} H_{6}\right)$ ou amônia borano $\left(\mathrm{NH}_{3}-\mathrm{BH}_{3}\right)[119,120]$. Com uma escolha apropriada das condições de crescimento, camadas ultrafinas e homogêneas de h-BN podem ser obtidas (1-5 camadas). Além disso, o 

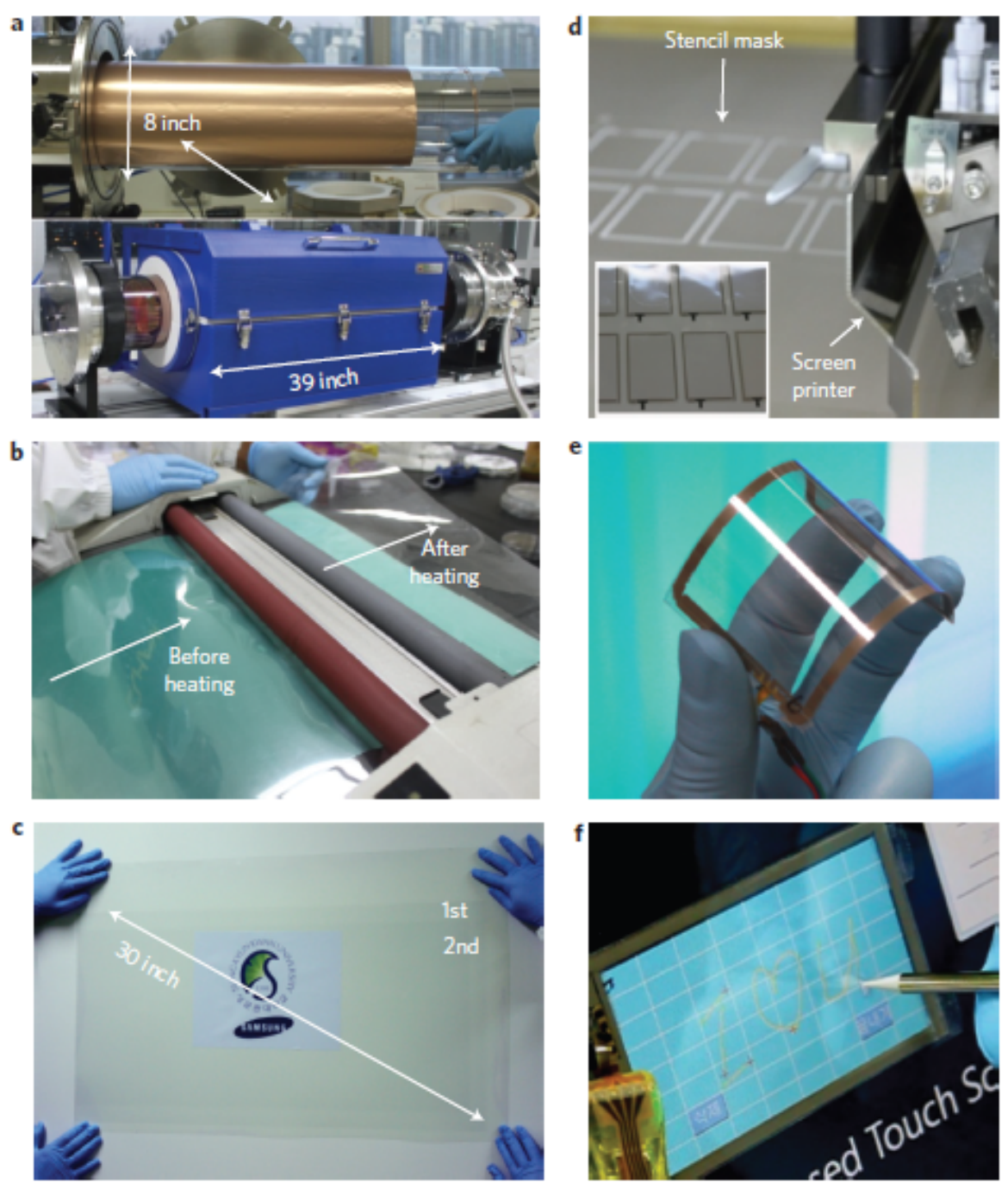

Figura 7.1: Fotografias do processo de fabricação de filmes de grafeno em larga escala. Imagem retirada da referência [118].

grafeno pode ser posteriormente crescido por CVD no topo dessas camadas de h-BN adsorvidas sobre o substrato metálico [121]. Esta é uma configuração ideal para dispositivos de efeito de campo.

Na figura 7.2(a), mostramos de forma esquemática a estrutura de um transistor de efeito de campo constituído de uma bicamada de grafeno como canal, suportada sobre um substrato de nitreto de boro hexagonal, sujeito a campos elétricos gerados pelos eletrodos de fonte e dreno e pelo top gate. Baseado nesse modelo simples de um FET, podemos destacar três seções fundamentais desse componente, que indicamos na figura 7.2(b,c,d): região onde o grafeno está suportada sobre o h-BN e em contato com os eletrodos de cobre, figura 7.2(c); h-BN servindo como dielétrico e substrato na presença de um top gate de cobre, figura 7.2(d); bicamada de 
(a)

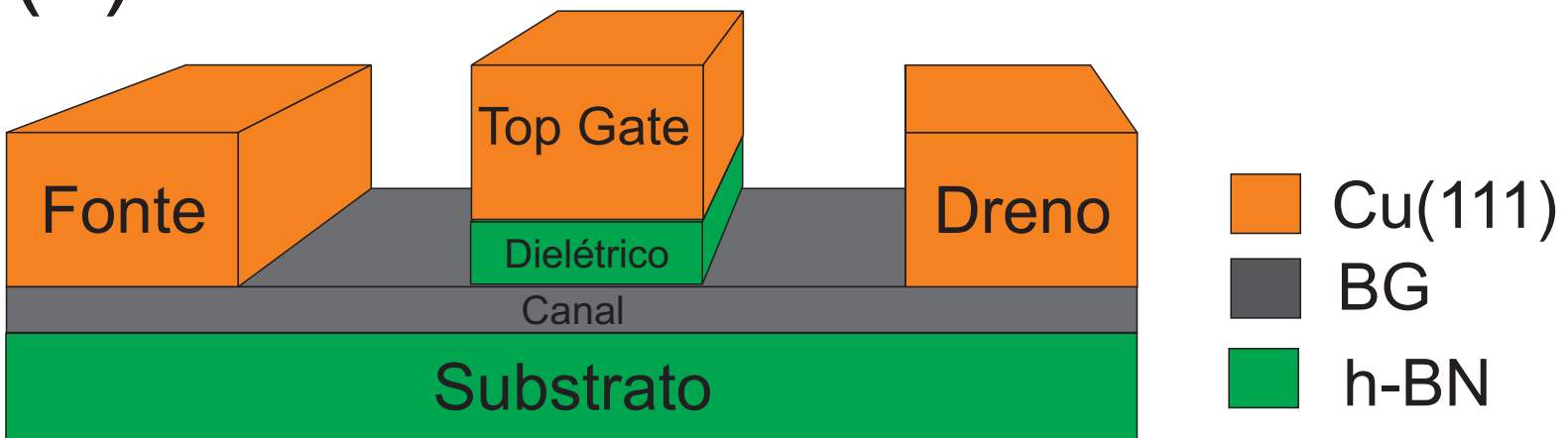

(b)

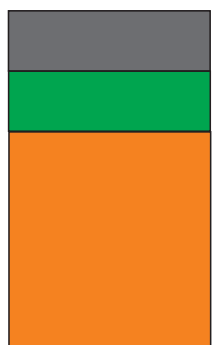

(c)

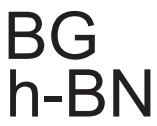

$\mathrm{Cu}(111)$
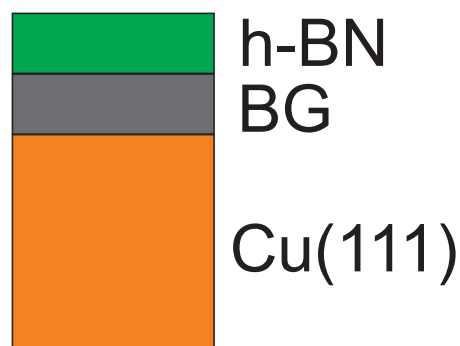

(d)
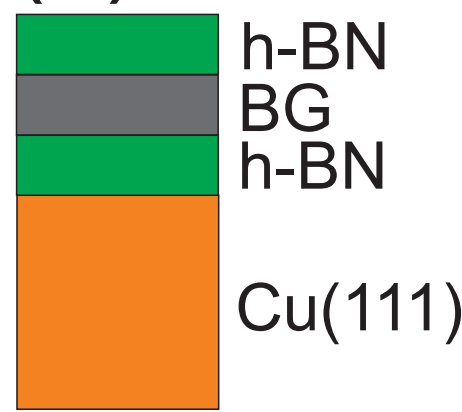

Figura 7.2: (a) Representação esquemática de um nanotransistor de bicamada de grafeno, onde são utilizados o nitreto de boro hexagonal como substrato e dielétrico e os eletrodos do sistema são constituídos por cobre. (b,c,d) Representação esquemática de possíveis configurações entre cobre/h-BN/BG que podem ser encontradas em um nanodispositivo. Estas seções serão estudadas separadamente.

grafeno sobre o h-BN e este sistema sobre o cobre, figura 7.2(b).

O objetivo deste capítulo é estudar separadamente cada componente que pode estar presente em um nanodispositivo de grafeno, onde são utilizados como substrato e dielétrico o h-BN e os contatos metálicos, neste caso, consistem de uma superfície de cobre(111). Na primeira seção estudaremos as propriedades eletrônicas e estruturais do contato bicamada de grafeno/cobre(111). Na seção seguinte, estudaremos o que ocorre com o h-BN quando depositado sobre a mesma superfície de cobre(111). Nas seções seguintes analisaremos as heteroestruturas indicadas na figura 7.2(b,c,d).

Nossas investigações foram baseadas em simulações $a b$ initio dentro do contexto da teoria do funcional da densidade (DFT) [37,40], utilizando o código SIESTA [122, 123]. Utilizamos a aproximação local da densidade (LDA) [66] para o termo de troca e correlação $\left(E_{x c}\right)$. A interação entre os elétrons de caroço e valência foram descritas utilizando pseudo potenciais de norma conservada de Troullier-Martins [124]. Utilizamos uma base dupla- $\zeta$ polarizada [125]. 
Para a integração no gride utilizamos 300Ry de mesh-cut-off. As estruturas foram consideradas relaxadas quando as forças residuais nos átomos fossem menores que $0.02 \mathrm{eV} / \AA$. Foi utilizado um total de 900 pontos- $k$ para a integração na zona de Brillouin da supercélula, utilizando o esquema de Monkhorst-Pack [71].

\subsection{Bicamada de grafeno sobre $\mathrm{Cu}(111)$}
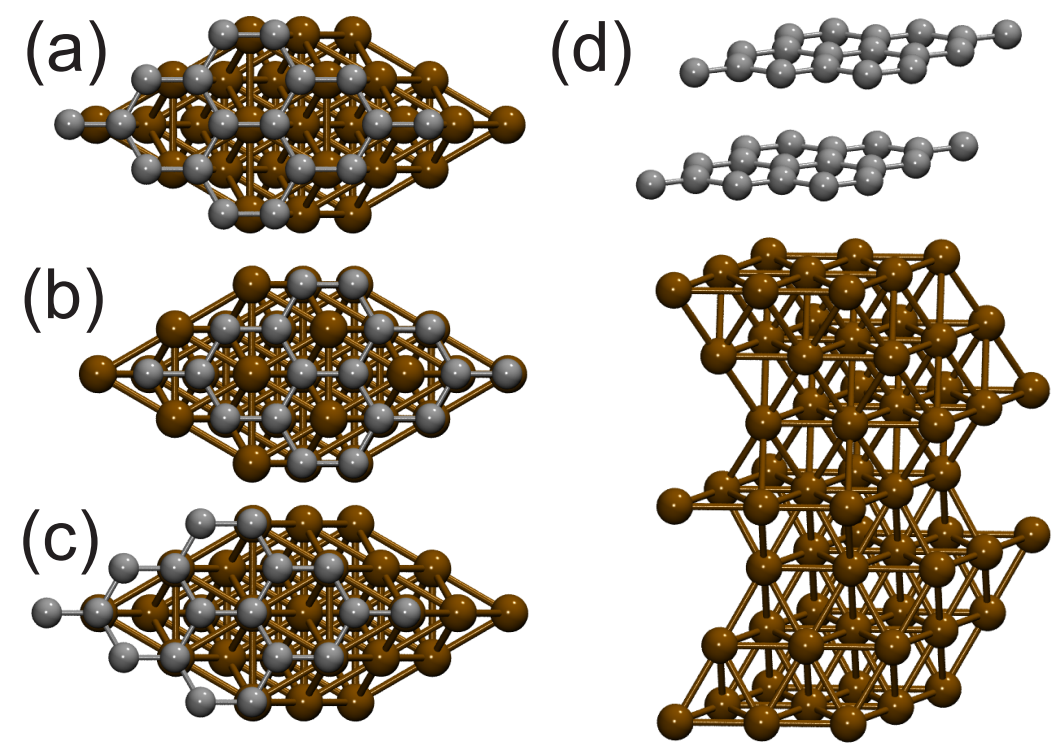

Figura 7.3: Empilhamentos utilizados nos cálculos das bicamadas de grafeno sobre a superfície (111) do Cobre. Para este sistema existem 3 possíveis configurações :(a) átomo de cobre no meio da ligação entre dois átomos de carbono; (b) átomo de cobre no meio do hexágono da camada de grafeno inferior; (c) átomo de cobre abaixo de um átomo de carbono da camada inferior.

Nesta seção, consideramos as propriedades estruturais e eletrônicas das bicamadas de grafeno depositadas sobre a superfície (111) do cobre. Na figura 7.3, mostramos as possíveis configurações da bicamada de grafeno depositada sobre a superfície de cobre. Em: (a) os átomos de cobre localizam-se no meio da ligação entre os dois átomos da rede do grafeno; (b) os átomos de cobre localizam-se no meio do hexágono do grafeno; (c) os átomos de cobre da superfície ficam localizados diretamente sobre um dos átomos de carbono da subrede do grafeno. A partir das geometrias da figura 7.3, realizamos cálculos de energia total, para determinar qual o tipo de empilhamento é o de mais baixa energia e qual a distância de equilíbrio entre a bicamada de grafeno e a superfície de cobre.

Na figura 7.4, mostramos os resultados da energética das bicamadas de grafeno suportadas sobre a superfície (111) do cobre. Observamos que a configuração de mais baixa energia é 


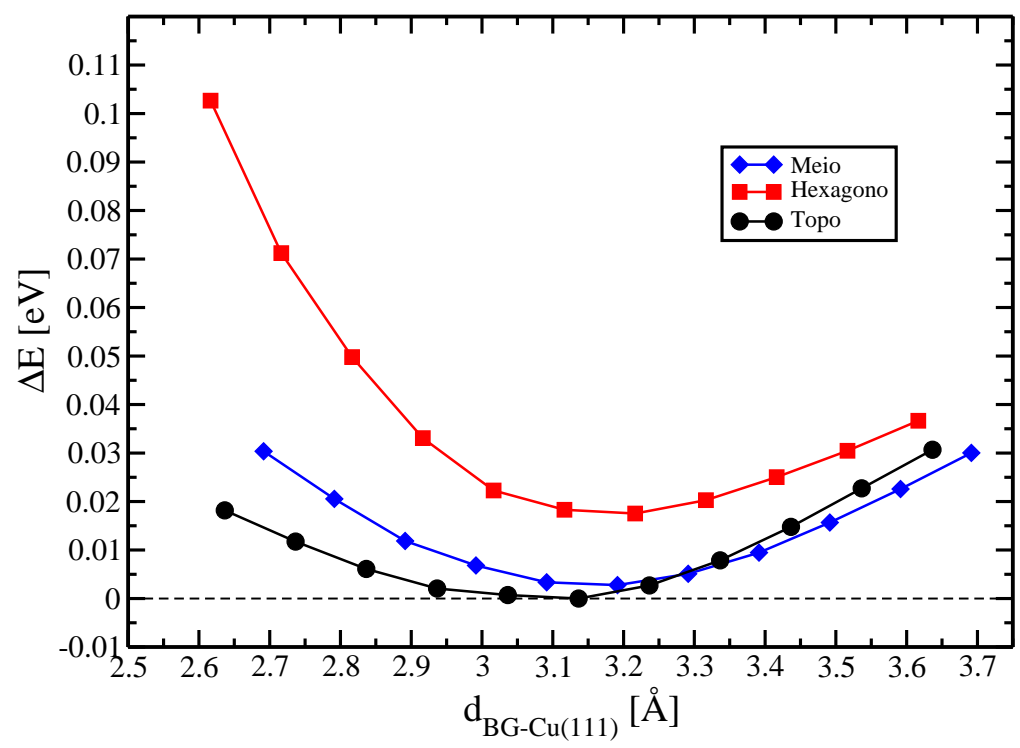

Figura 7.4: Curvas de energia potencial para os três possíveis empilhamentos das bicamadas de grafeno sobre a superfície (111) do cobre.

quando um átomo de carbono está localizado diretamente acima de um átomo de cobre da superfície, conforme mostrado na figura 7.3(c). A distância de equilíbrio é de $\approx 3.13 \AA$. Um ponto importante, é que a bicamada de grafeno fica adsorvida fisicamente sobre a superfície, não existindo nenhuma ligação química entre os dois sistema.

Partindo da configuração de mais baixa energia, realizamos cálculos de estrutura eletrônica para as bicamadas de grafeno sobre o $\mathrm{Cu}(111)$, na presença e ausência de campos elétricos externos aplicados. Na figura 7.5(a,d), mostramos a estrutura de bandas de uma bicamada de grafeno sobre a superfície de cobre(111) sem a presença de campo elétrico. As linhas em preto representam os estados híbridos do contato bicamada de grafeno/ $\mathrm{Cu}(111)$, enquanto as linhas em azul mostram as bandas pertencentes à bicamada de grafeno. Primeiramente, observamos que devido a pequena hibridização entre a bicamada e a superfície de cobre, ocorre um pequeno shift dos estados da bicamada para baixo do nível de Fermi de aproximadamente $-0.3 \mathrm{eV}$, resultando em uma dopagem tipo $n$ da bicamada. Esta dopagem ocorre também devido a diferença entre as funções trabalho da BG em relação à superfície de cobre, onde ocorre uma transferência de elétrons do cobre para a bicamada. Isso cria uma região de depleção entre os sistemas, gerando um campo elétrico efetivo dentro da bicamada, que é responsável pela abertura de um gap de energia de aproximadamente $72 \mathrm{meV}$, mesmo a campo externo zero.

Quando um campo elétrico positivo é aplicadoํㄱ figuras 7.5(b,c), os elétrons inicialmente

\footnotetext{
${ }^{1}$ Positivo significa um campo aplicado na direção do cobre para a bicamada e negativo da bicamada para o
} 

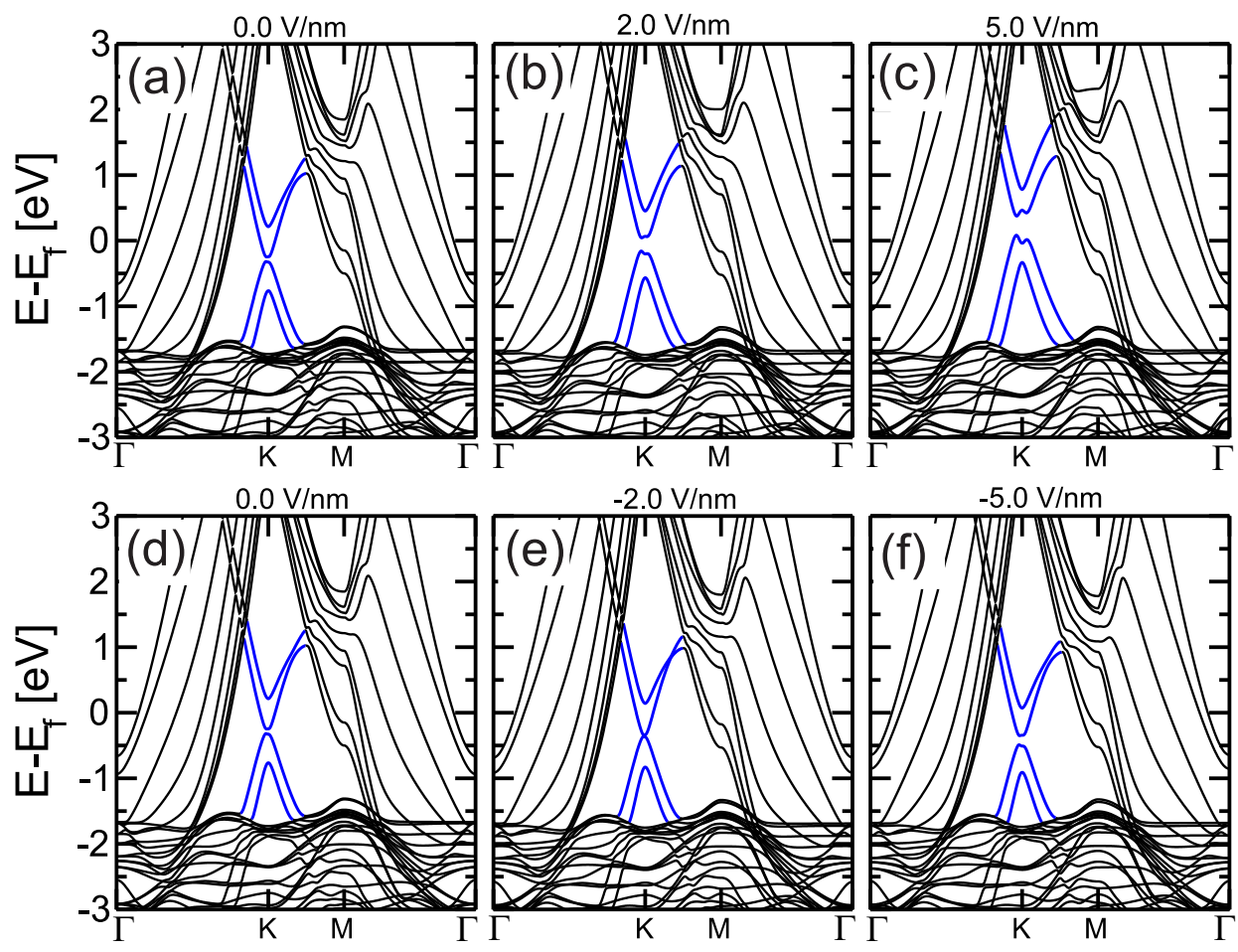

Figura 7.5: Evolução da estrutura de bandas de uma bicamada de grafeno sobre cobre(111). Estruturas de bandas para o sistema (a,d) sem campo elétrico externo e com campo elétrico externo aplicado de (b) $+2.0 \mathrm{~V} / \mathrm{nm}$, (c) $+5.0 \mathrm{~V} / \mathrm{nm}$, (e) $-2.0 \mathrm{~V} / \mathrm{nm}$ e (f) $-5.0 \mathrm{~V} / \mathrm{nm}$.

transferidos do metal para a bicamada retornam gradualmente para o cobre, levando a bicamada de uma dopagem tipo $n$ para uma dopagem tipo $p$, passando por um estado neutro. Ocorre também um aumento do valor do gap de energia conforme podemos observar na figura 7.6. Quando a direção do campo elétrico é invertida, observamos que a bicamada de grafeno irá sempre ficar dopada tipo $n$, figura 7.5(e,f). Algo importante a notar, é que o gap de energia neste sistema, primeiramente diminui, indo para um valor mínimo, e depois volta a aumentar, como mostramos na figura 7.6. Isto ocorre, pois o campo elétrico induzido na região de depleção criada entre os sistemas é anulado pelo campo externo aplicado.

\subsection{Nitreto de Boro Hexagonal sobre $\mathrm{Cu}(111)$}

Nesta seção investigamos as propriedades estruturais e eletrônicas do nitreto de boro hexagonal depositado sobre a superfície (111) do Cobre. Na figura 7.7, mostramos as possíveis configurações da camada inferior de h-BN depositado sobre a superfície de cobre. Em: (a) os átomos de cobre da superfície ficam localizados no meio dos hexágonos da camada inferior de cobre. 

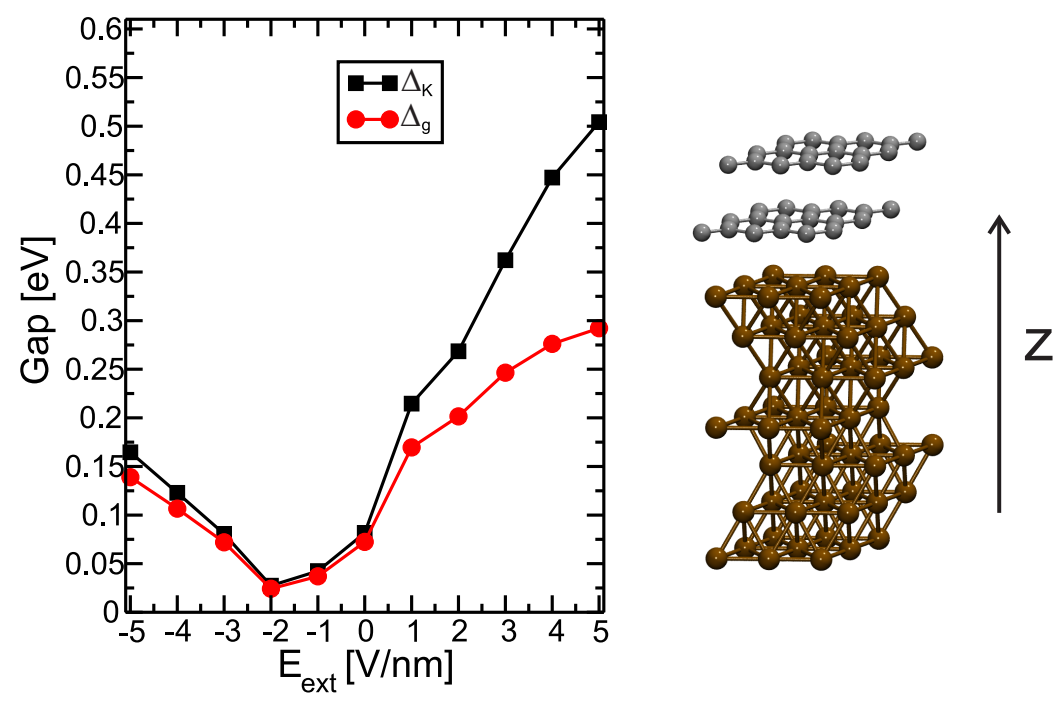

Figura 7.6: Gap de energia induzido na bicamada de grafeno. Em (a) $\Delta_{g}$, menor distância entre as bandas das bicamadas; (b) $\Delta_{K}$, gap no ponto de alta simetria $K$ do grafeno.

h-BN; (b) os átomos de cobre ficam abaixo dos átomos de nitrogênio do h-BN; (c) os átomos de cobre ficam abaixo dos átomos de boro do h-BN. A partir das geometrias da figura 7.7, realizamos cálculos de energia total, para determinar qual o tipo de empilhamento é o de mais baixa energia e qual a distância de equilíbrio entre as camadas de h-BN e a superfície de cobre.
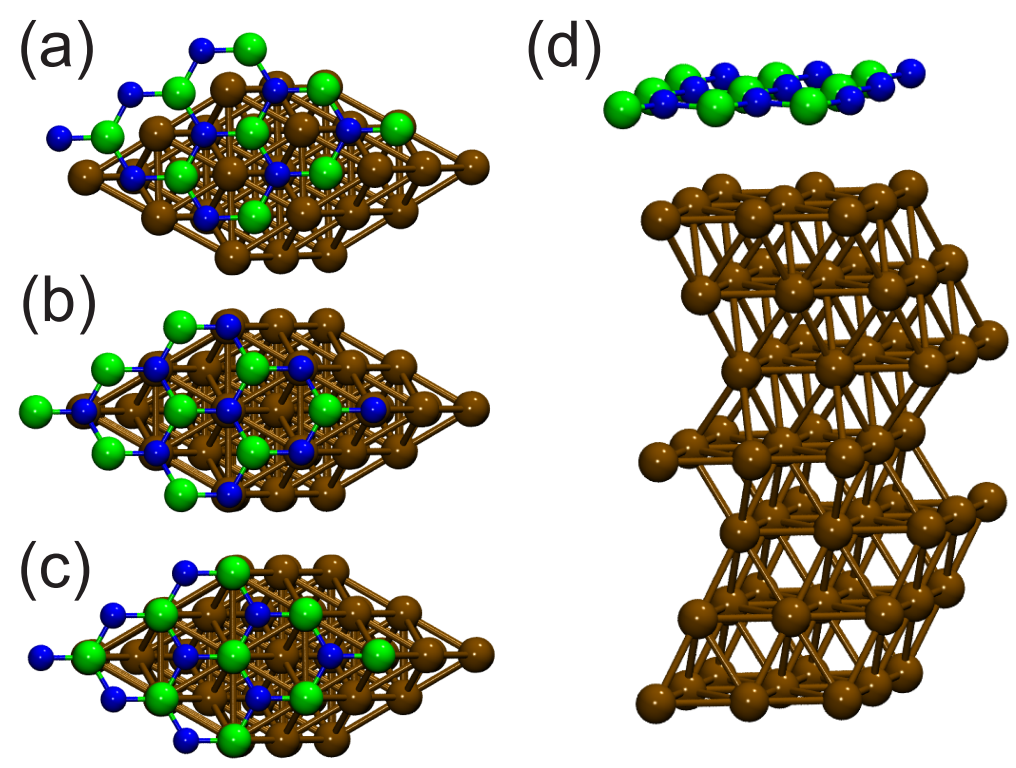

Figura 7.7: Empilhamentos utilizados nos cálculos do h-BN sobre a superfície (111) do Cobre. (a) Centro do hexágono do h-BN sobre um átomo de cobre; (b) átomo de nitrogênio sobre um átomo de cobre; (c) átomo de boro sobre um átomo de cobre; (d) vista lateral da estrutura contendo uma única camada de h-BN depositada sobre uma superfície de cobre com 7 planos atômicos de cobre. 
Na figura 7.8 mostramos os resultados dos cálculos de energia total para os sistemas da figura 7.7. A configuração de energia mais baixa foi obtida com o nitrogênio sobre os átomos de cobre. A distância de equilíbrio calculada foi de $\approx 2.93 \AA$.

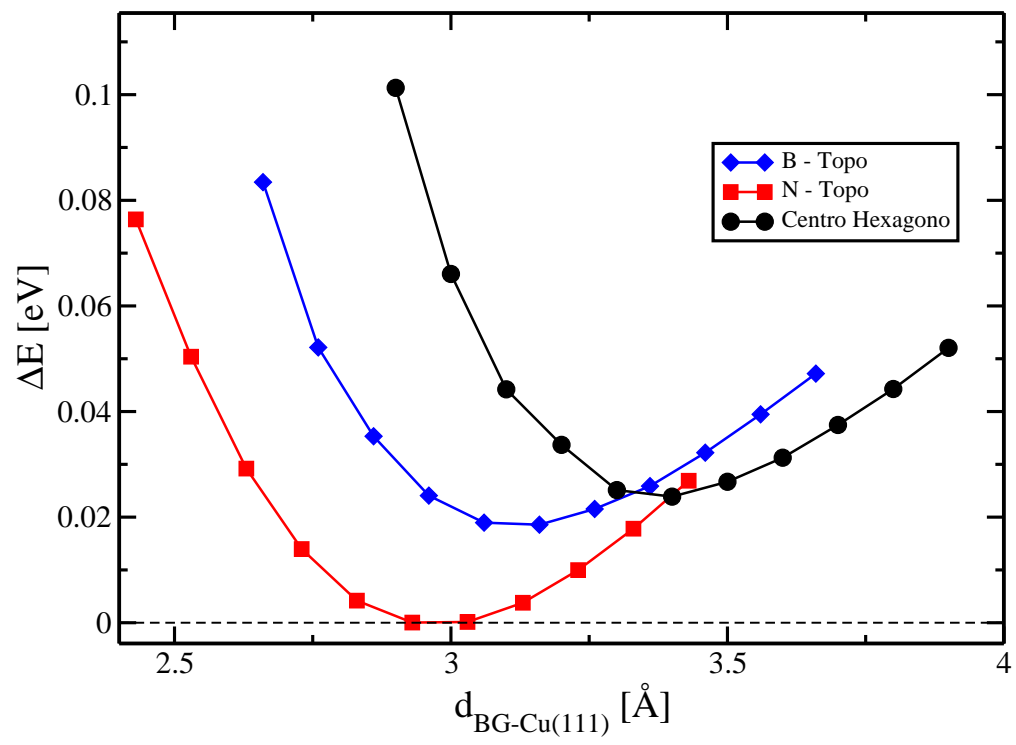

Figura 7.8: Curvas de energia potencial para os três possíveis empilhamentos do h-BN sobre a superfície (111) do cobre.

A partir da configuração de mais baixa energia, estudamos o comportamento da estrutura eletrônica para sistemas compostos de até quatro camadas de h-BN. Determinamos qual a influência de um campo elétrico externo na estrutura eletrônica desses sistemas. A evolução da estrutura eletrônica em função do número de camadas e do campo elétrico externo é apresentada na figura 7.9.

Podemos observar que o comportamento das bandas das camadas de nitreto de boro hexagonal é muito semelhante ao observado no capítulo 6, onde devido ao gradiente de potencial gerado perpendicularmente ao sistema, ocorre um desdobramento das bandas do h-BN. Os estados pertencentes ao isolante tendem a criar um canal de condução em função do campo elétrico, e quanto mais camadas de h-BN mais sensível é o sistema à influência do campo.

\subsection{Interações entre BG/Cobre(111)/h-BN}

Nesta seção, iremos estudar a estrutura eletrônica das componentes de um nanotransistor de bicamada de grafeno, descritos na figura 7.2. 
(a)
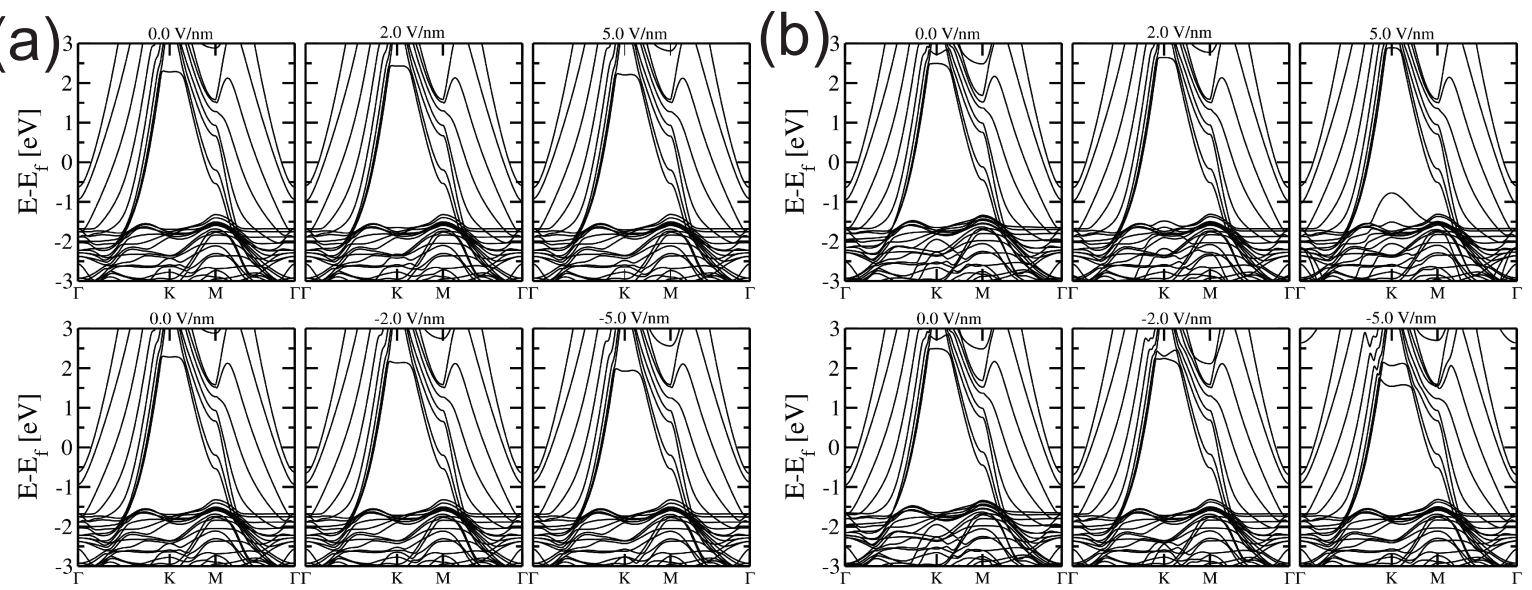

(c)
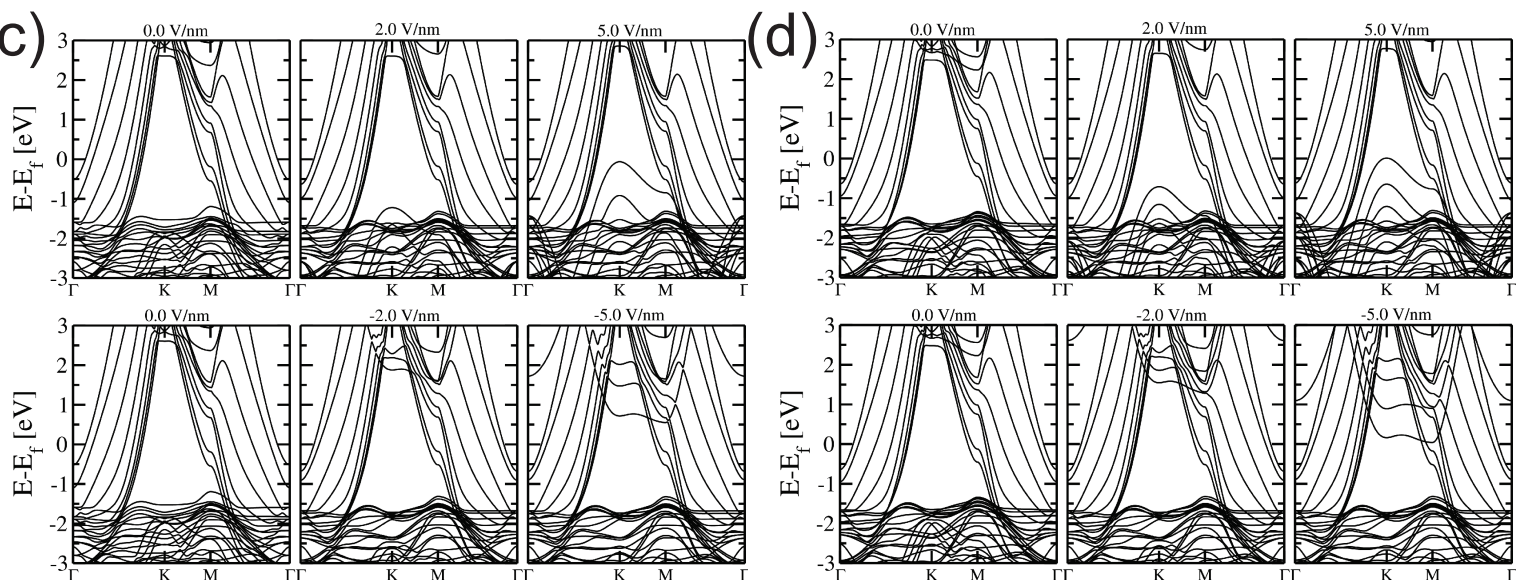

Figura 7.9: Evolução da estrutura de bandas para o h-BN sobre cobre(111) para (a) 1 camada de hBN; (b) 2 camadas de h-BN; (c) 3 camadas de h-BN; (d) 4 camadas de h-BN. Em todas as figuras apresentamos os resultados para o sistema na ausência de campo elétrico externo e na presença de campo - quadros superiores campos positivos e quadros inferiores campos negativos.

\subsection{1 $\mathrm{Cu}(111) / \mathrm{h}-\mathrm{BN} /$ Bicamada}

Agora, analisamos o sistema descrito em 7.2(b). Determinamos qual a influência do número de camadas de h-BN e do campo elétrico na estrutura eletrônica deste sistema composto. Os sistemas estudados consistem em uma bicamada de grafeno suportada sobre $N(N=1,2,3,4)$ camadas de h-BN e este sistema sobre uma superfície (111) de cobre, como mostrados na figura 7.10. O contato metálico é composto de 7 planos atômicos de cobre, onde a superfície exposta e em contato com o h-BN é a (111). Escolhemos o empilhamento de mais baixa energia para a interface entre o cobre e o nitreto de boro hexagonal, onde os átomos de nitrogênio estão sobre os átomos de cobre.

Na figura 7.11, apresentamos o comportamento da estrutura de bandas dos sistemas mostrados na figura 7.10. Primeiramente, podemos observar que a campo zero ocorre a abertura de 


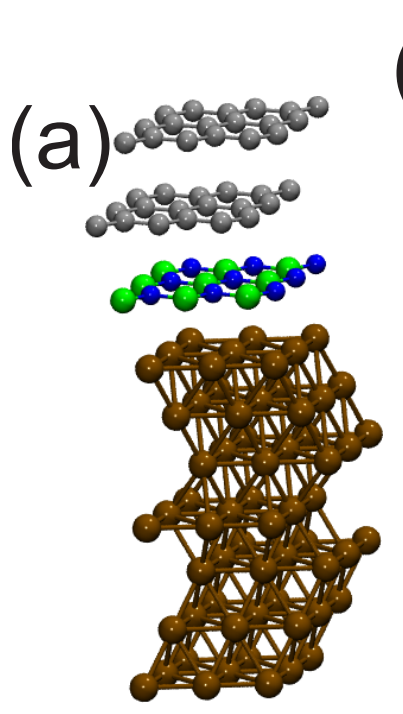

(b)

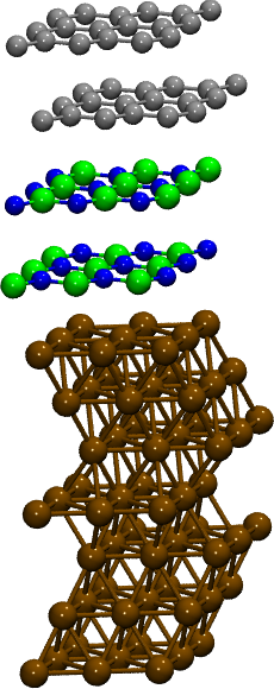

(c)

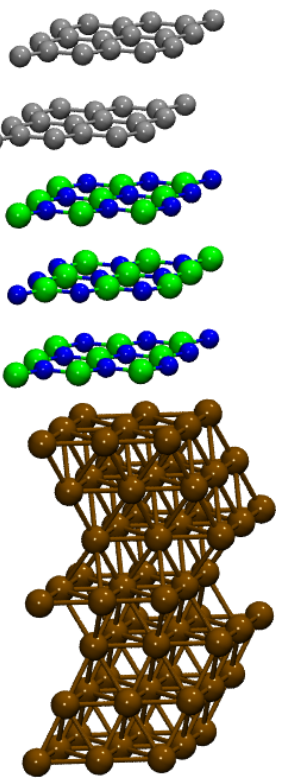

(d)

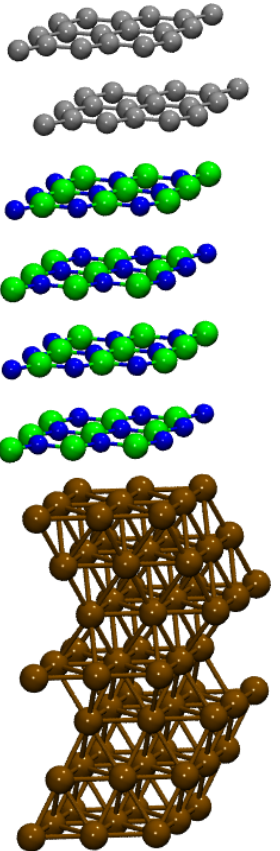

Figura 7.10: Representação das geometrias utilizadas. (a) 1 camada de h-BN; (b) 2 camadas de h-BN (c) 3 camadas de h-BN; (d) 4 camadas de h-BN.

um gap de energia na BG, como já havíamos observado anteriormente no sistema bicamada sobre cobre ou mesmo para as bicamadas sobre o h-BN.

Um segundo ponto importante é que o comportamento observado com respeito à variação da dopagem da BG de tipo $n$ para tipo $p$ somente para campos elétricos positivos também é observado e este comportamento é independente do número de camadas de h-BN. Finalmente, notamos que todas as estruturas de bandas são muito semelhantes em função do número de camadas de h-BN, não ocorrendo a adição de nenhum estado pertencente ao h-BN na região do nível de Fermi do sistema. Desta maneira, o h-BN comporta-se como um excelente isolante entre o cobre e a bicamada, onde independente do número de camadas de h-BN e do campo elétrico aplicado, não é criado um canal de condução pelo h-BN.

Na figura 7.12, mostramos o comportamento do gap de energia, $\Delta_{g}$, induzido na bicamada de grafeno em função do campo elétrico e do número de camadas de h-BN. Primeiramente, observamos que a variação do gap na bicamada de grafeno é muito semelhante ao observado na figura 7.6. Um outro ponto importante é que quanto mais camadas de h-BN menor é a intensidade do campo necessária para se obter o valor mínimo para o gap de energia na bicamada de grafeno. 
(a)
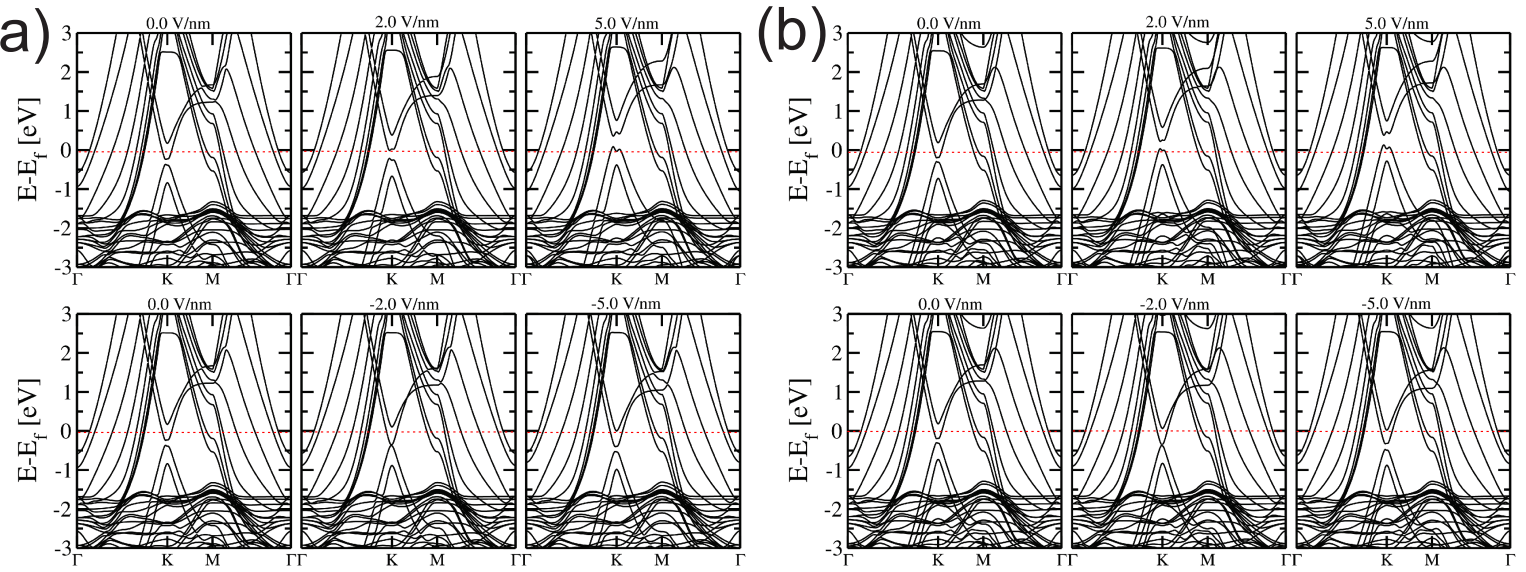

(c)

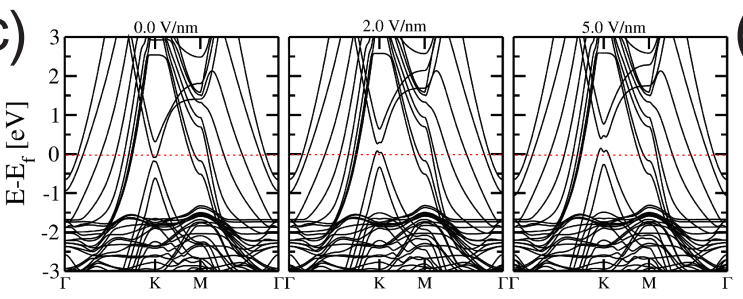

(d)
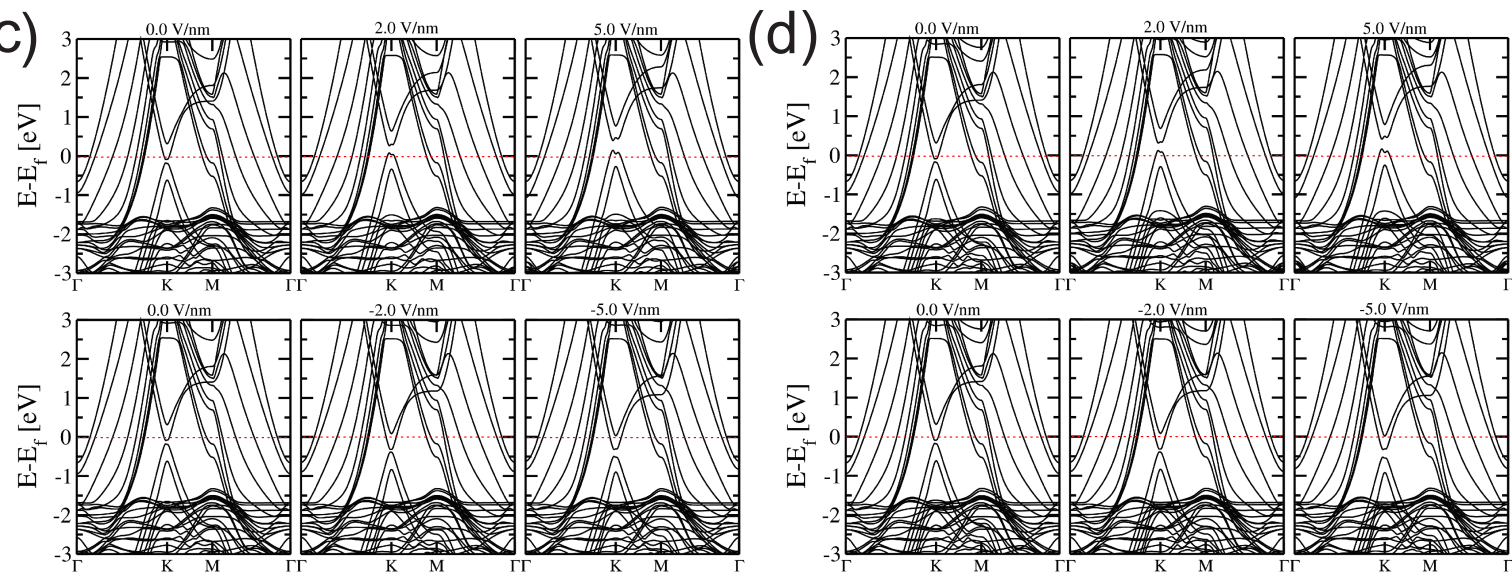

Figura 7.11: Evolução da estrutura de bandas para o sistema $\mathrm{Cu}(111) / \mathrm{h}-\mathrm{BN} / \mathrm{BG}$ para: (a) 1 camada de h-BN; (b) 2 camadas de h-BN; (c) 3 camadas de h-BN; (d) 4 camadas de h-BN. Em todas as figuras apresentamos os resultados para o sistema na ausência de campo elétrico externo e na presença de campo - quadros superiores campos positivos e quadros inferiores campos negativos.

\subsection{2 $\mathrm{Cu}(111) /$ Bicamada/h-BN}

Continuando com o estudo das componentes de um nanotransistor, iremos estudar agora o sistema composto de uma bicamada de grafeno suportada sobre uma superfície de cobre e coberta por h-BN. Este sistema foi descrito na figura 7.2(c). Assim como os outros casos, estudamos a influência da aplicação de um campo elétrico externo e do número de camadas de h-BN sobre a bicamada de grafeno. Na figura 7.13, mostramos as geometrias dos sistemas utilizados.

Na figura 7.14, apresentamos os resultados para a estrutura eletrônica na presença e ausência de campo externo. O comportamento da estrutura eletrônica deste sistema não apresenta mudanças significativas se comparados com os outros sistemas estudados anteriormente. $\mathrm{O}$ comportamento global deste sistema pode ser visualizado como sendo uma combinação dos 


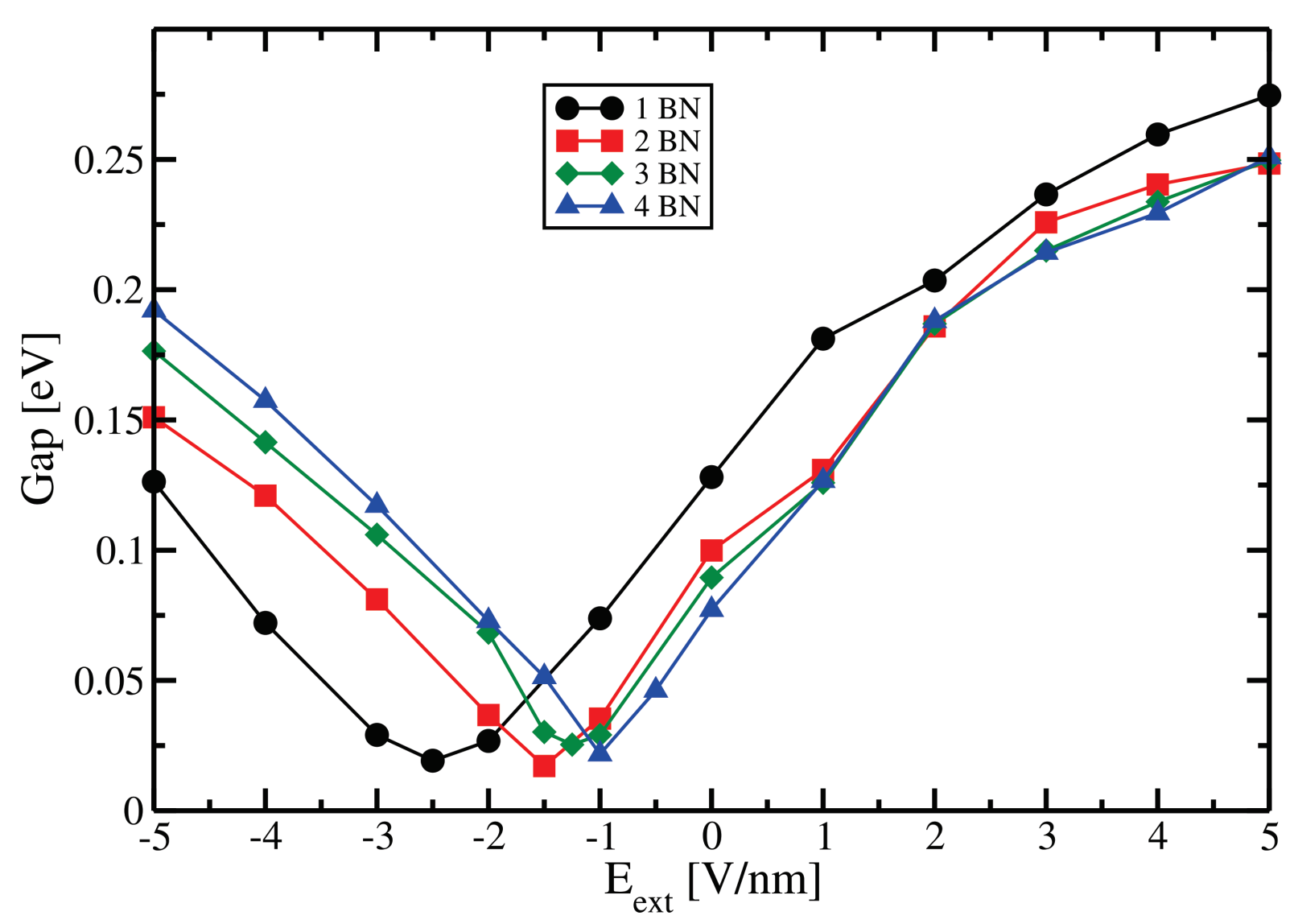

Figura 7.12: Gap de energia induzido na bicamada de grafeno em função do campo elétrico externo para: (a) $\mathrm{Cu}(111) / 1 \mathrm{~h}-\mathrm{BN} / \mathrm{BG}$; (b) $\mathrm{Cu}(111) / 2 \mathrm{~h}-\mathrm{BN} / \mathrm{BG}$; (c) $\mathrm{Cu}(111) / 3 \mathrm{~h}-\mathrm{BN} / \mathrm{BG}$; (d) $\mathrm{Cu}(111) / 4 \mathrm{~h}-\mathrm{BN} / \mathrm{BG}$. Este gap é calculado como sendo a menor distância entre as bandas das bicamadas de grafeno.

dois sistemas isolados: $\mathrm{Cu}(111) / \mathrm{BG}$ e $\mathrm{Cu}(111) / \mathrm{h}-\mathrm{BN}$. Ou seja, é possível tanto modificar a dopagem da bicamada de grafeno através da aplicação de campos elétricos externos como controlar o valor do gap de energia na bicamada. Um outro ponto que vale ser mencionado, é que quanto mais camadas de h-BN mais sensível fica o sistema na presença de campos elétricos. Nesta configuração ocorre a inserção de níveis do nitreto de boro na região do nível de Fermi do sistema, levando a abertura de um canal de condução através do dielétrico.

\subsection{3 $\mathrm{Cu}(111) / \mathrm{hBN} /$ Bicamada/hBN}

Para finalizarmos o estudos das componentes isoladas de um nanotransistor, estudamos a situação onde o nitreto de boro hexagonal é utilizado como substrato e dielétrico ao mesmo tempo e, e este sistema está em contato com uma superfície de cobre. Este sistema foi apresentado na figura 7.2(d). Utilizamos configurações simétricas em relação ao número de camadas de nitreto de boro, ou seja, o mesmo número de camadas utilizadas como substrato foi também 


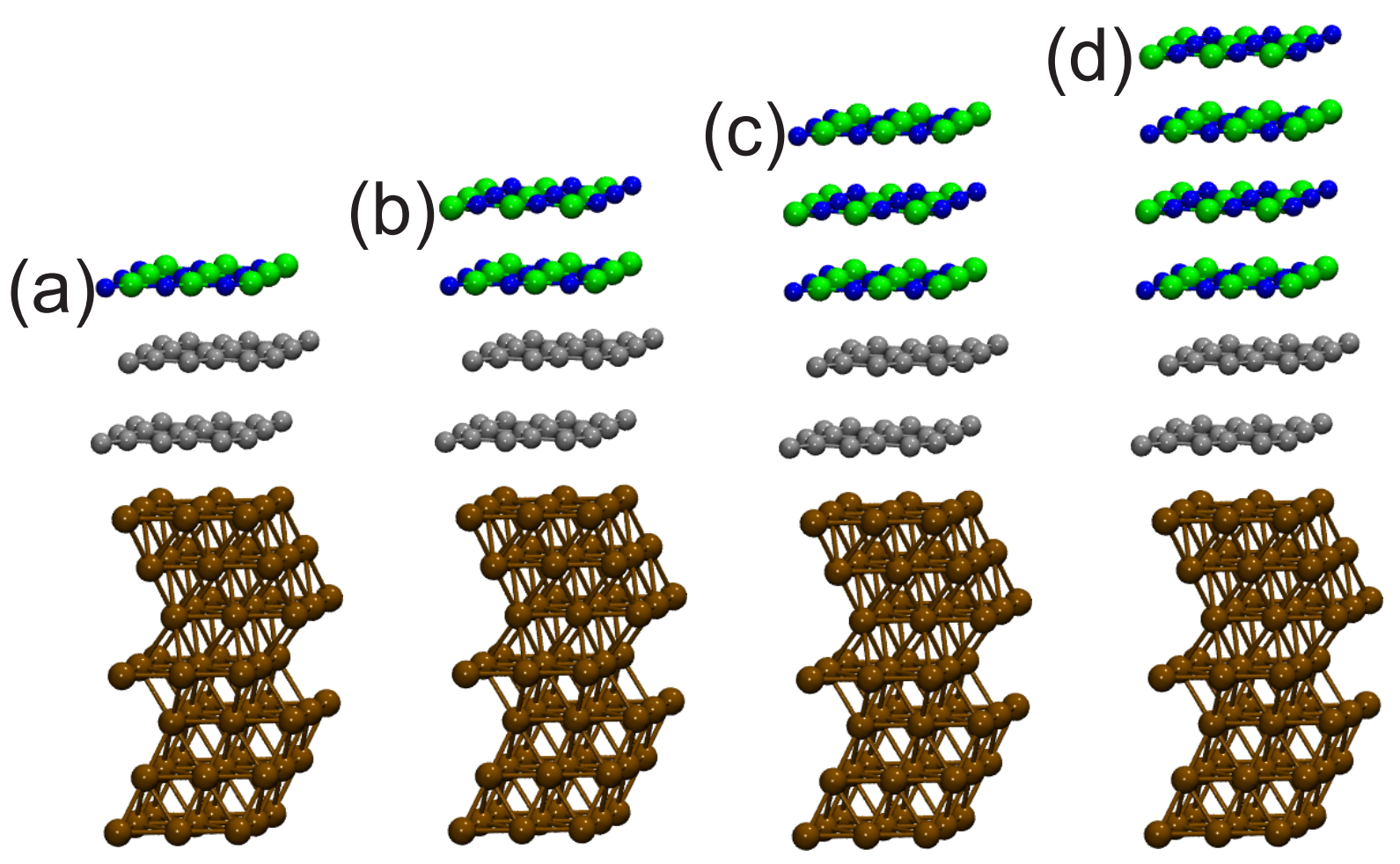

Figura 7.13: Representação das geometrias utilizadas. (a) 1 camada de h-BN; (b) 2 camadas de h-BN; (c) 3 camadas de h-BN; (d) 4 camadas de h-BN.

utilizada como dielétrico. Utilizamos uma bicamada de grafeno entre 1 e 3 camadas de h-BN e este sistema depositado sobre uma superfície de cobre(111). Na figura 7.15, apresentamos dois resultados representativos deste sistema.

Notamos que a dopagem intrínseca, tipo $n$, das bicamadas de grafeno continua ocorrendo. Observamos que o controle da dopagem e do valor do gap de energia através da variação dos campos elétricos externos só é possível para o sistema com uma única camada de h-BN, ou seja, com uma única camada é possível mudar o tipo de portadores na bicamada, indo de tipo $n$ para tipo $p$. Existe também a possibilidade do controle contínuo do gap de energia na bicamada de grafeno, como mostramos anteriormente. Entretanto, quando utilizamos 3 camadas de h-BN o gap de energia varia muito pouco quando aplicamos campos elétricos positivos, mantendose praticamente o mesmo com uma variação de $5 \mathrm{~V} / \mathrm{nm}$ para campos positivos. Um outro ponto, na presença de campos elétricos positivos, a partir de um campo elétrico de aproximadamente $+1.0 \mathrm{~V} / \mathrm{nm}$, as bicamadas de grafeno ficam neutras, independentemente da intensidade do campo elétrico aplicado. 
(a)
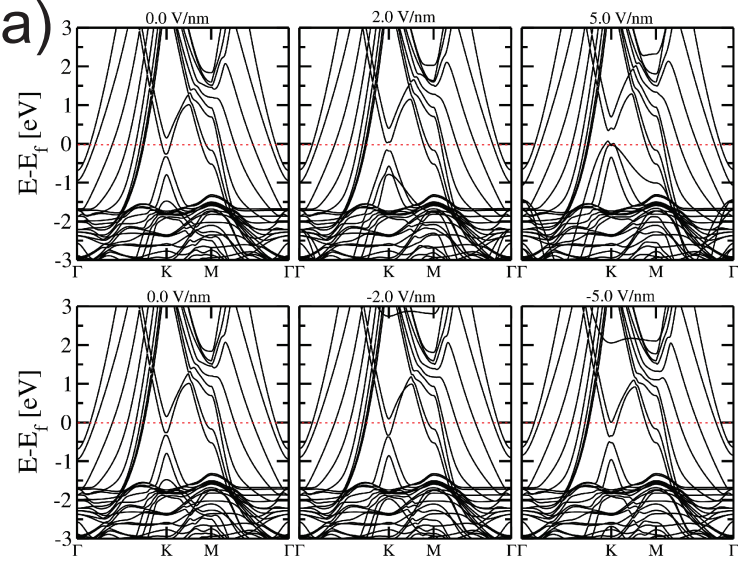

(c)
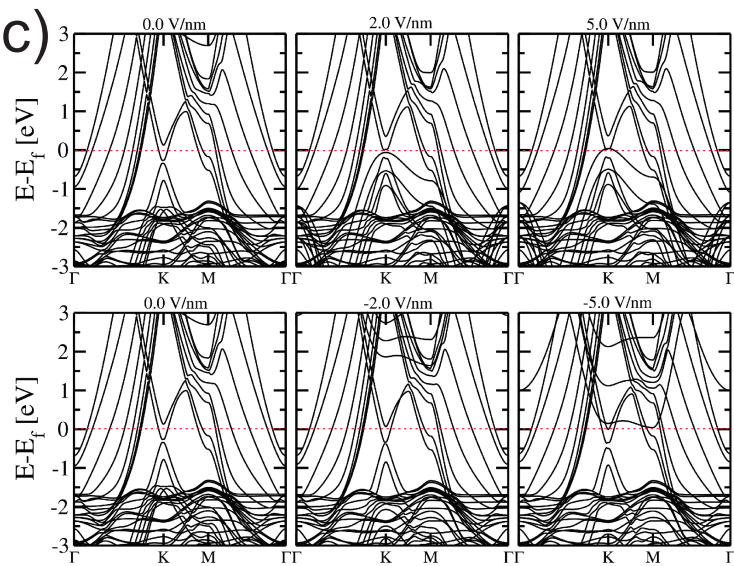

(b)
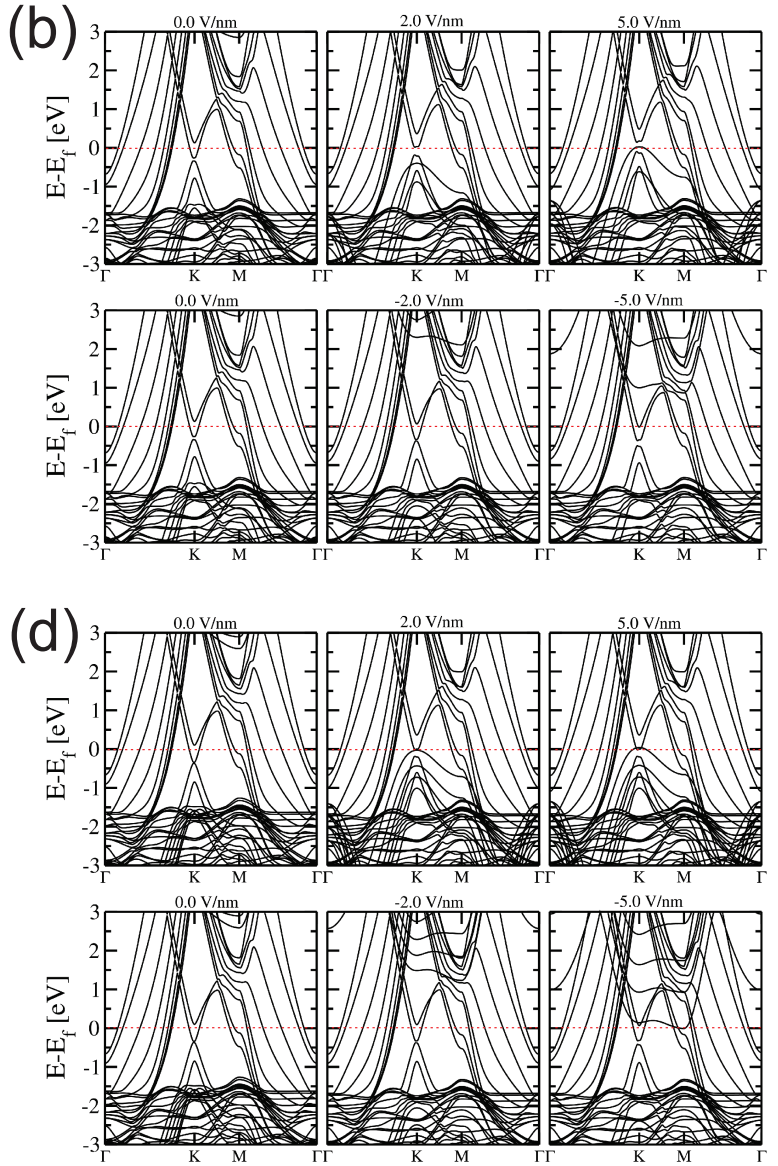

Figura 7.14: Evolução da estrutura de bandas para o sistema $\mathrm{Cu}(111) / \mathrm{BG} / \mathrm{h}-\mathrm{BN}$ para: (a) 1 camada de h-BN; (b) 2 camadas de h-BN; (c) 3 camadas de h-BN; (d) 4 camadas de h-BN. Em todas as figuras apresentamos os resultados para o sistema na ausência de campo elétrico externo e na presença de campo - quadros superiores campos positivos e quadros inferiores campos negativos.

\subsection{Conclusões}

Observamos que em todos os sistemas considerados em nossas investigações, independentemente do número de camadas de h-BN no sistema na ausência de campo elétrico, as bicamadas de grafeno ficam sempre dopadas tipo $n$. Mostramos que é possível controlar o tipo de dopagem das bicamadas, variando de tipo $n$ a tipo $p$, passando por um estado neutro, para todas as configurações, exceto nos sistemas $\mathrm{Cu}(111) / \mathrm{h}-\mathrm{BN} / \mathrm{BG} / \mathrm{h}-\mathrm{BN}$ com mais de 1 camada de h-BN.

Verificamos que é possível controlar o valor do gap de energia nas bicamadas de grafeno através de um campo elétrico externo, onde sintonizando a intensidade e a direção do campo conseguimos tanto zerar o gap induzido pelo campo elétrico gerado na região de depleção 

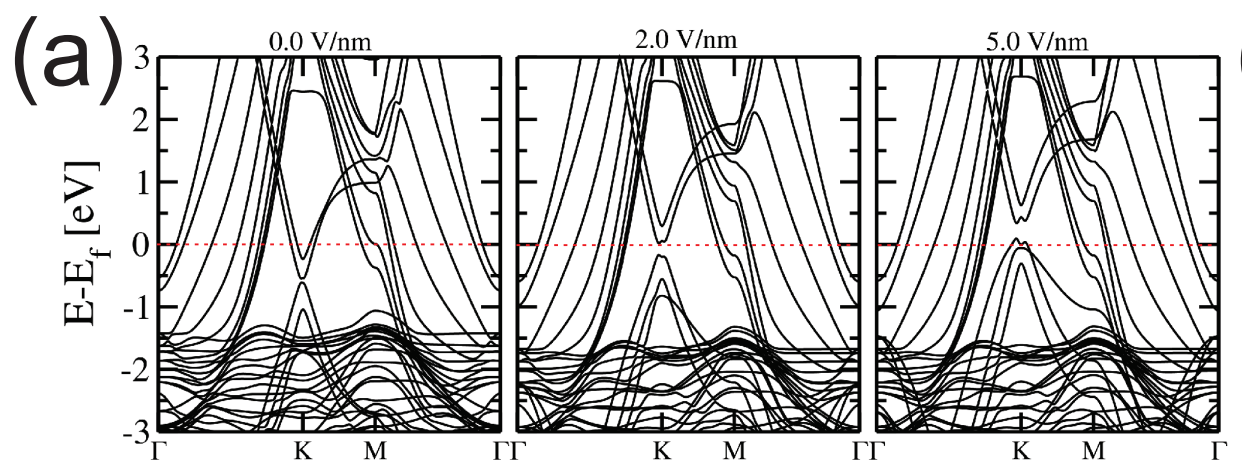

(b)
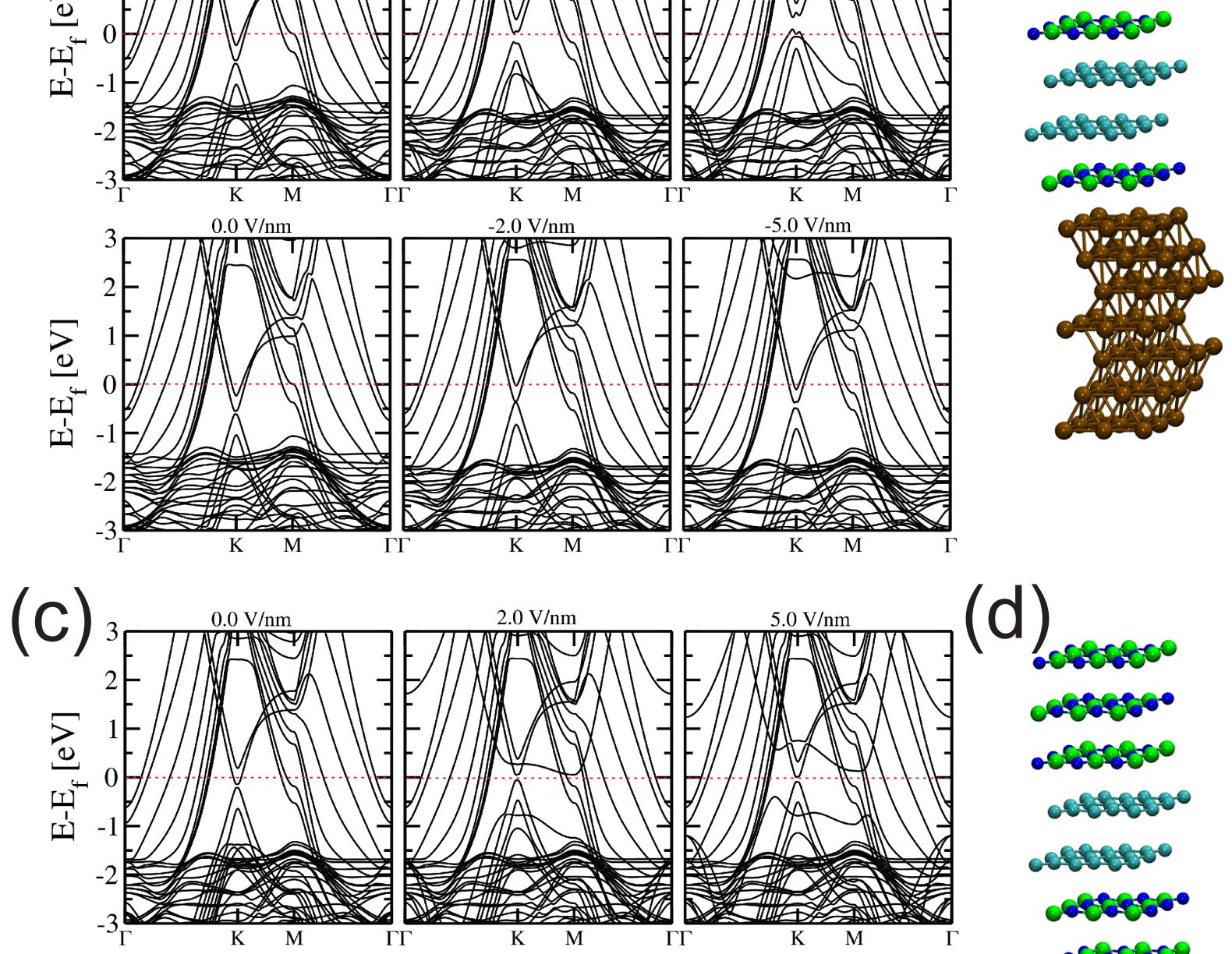

(d)
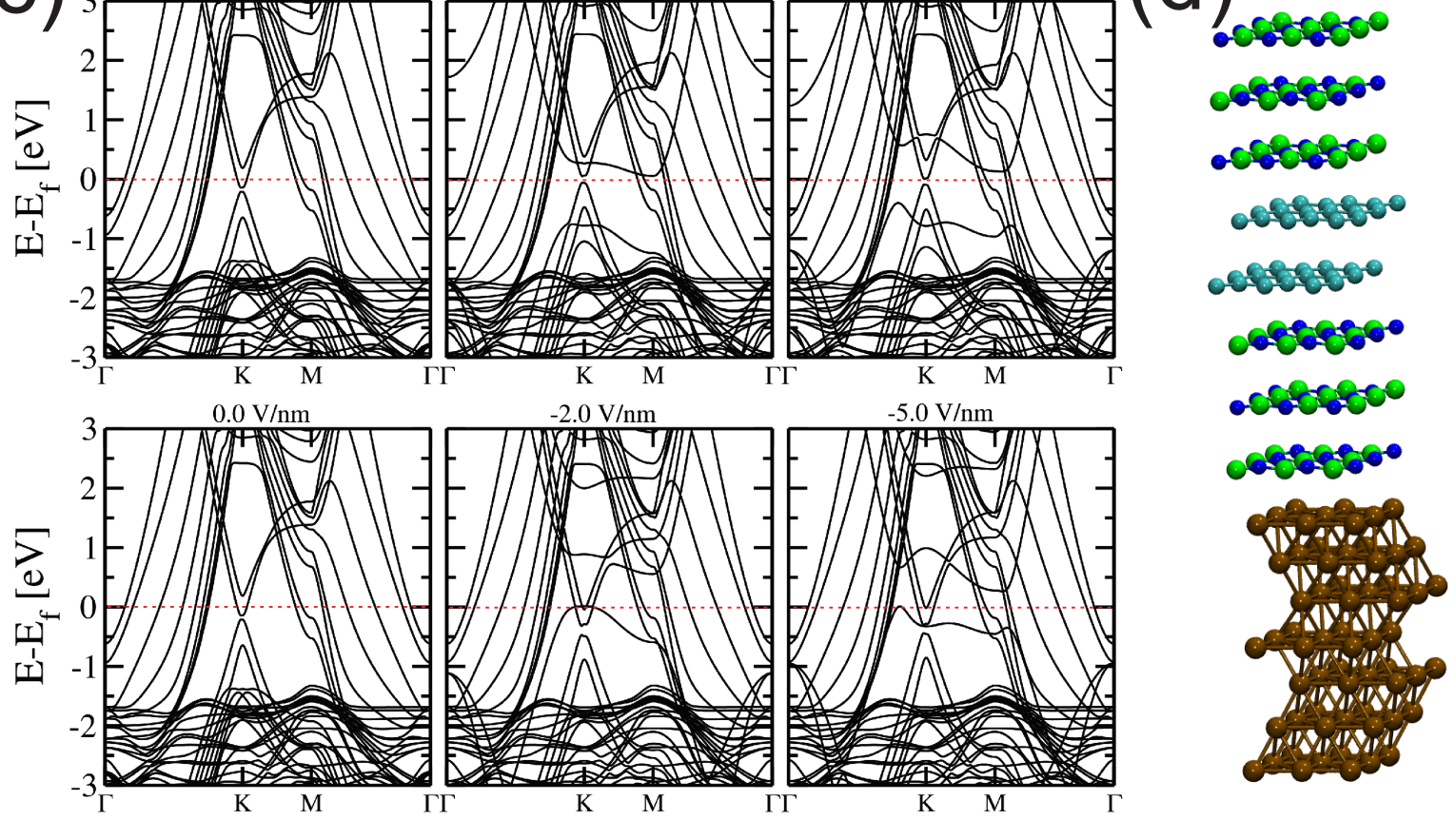

Figura 7.15: Evolução da estrutura de bandas para o sistema $\mathrm{Cu}(111) / \mathrm{h}-\mathrm{BN} / \mathrm{BG} / \mathrm{h}-\mathrm{BN}$ em função do campo elétrico aplicado para: (a) 1 camada de h-BN; (b) 3 camadas de h-BN. Em todas as figuras apresentamos os resultados para o sistema na ausência de campo elétrico externo e na presença de campo - quadros superiores campos positivos e quadros inferiores campos negativos. 
criada na interface entre o cobre e o restante do sistema, como seria possível aumentar o valor deste gap de energia.

Finalmente, dentre todos os sistemas estudados, no sentido de aplicação direta em um dispositivo, concluímos que a melhor configuração é a $\mathrm{Cu}(111) / \mathrm{h}-\mathrm{BN} / \mathrm{BG}$, representada na figura 7.2(c). Neste sistema, independente do número de camadas de h-BN, é possível controlar o valor do gap de energia e tipo de dopagem, sem a adição de estados vindos do h-BN, criando um canal de condução através do dielétrico. 


\section{Capítulo 8}

\section{Propriedades de transporte de nanofitas de grafeno: pristinas e dopadas com boro e nitrogênio}

As nanofitas de grafeno(GNR), que são finas tiras de grafeno [20,126,127], tem sido extensivamente estudadas, não somente devido ao interesse em suas propriedades físicas intrigantes e interessantes, mas também por apresentarem um grande potencial para aplicações tecnológicas $[128,129]$. Propriedades tais como: baixo ruído no transporte, altos valores de magnetoresistência e alta condutividade elétrica e térmica, fazem das nanofitas uma possível alternativa para dispositivos em geral $[29,54,130,131]$.

As propriedades eletrônicas das nanofitas dependem fortemente da estrutura cristalina de suas bordas (armchair ou zigzag). Nanofitas de grafeno com orientação das bordas bem controladas tem sido fabricadas utilizando litografia por feixe eletrônico, através de técnicas de STM [132]. Existem vários outros métodos de fabricação, como a síntese molecular, que fornece nanofitas com bordas altamente controladas e sem a presença de defeitos [33]. Nanofitas com bordas zigzag apresentam um ordenamento magnético devido a uma polarização de spin nas bordas. Nesse caso, existe um grande interesse para a utilização das GNR como componentes para spintrônica [133-135].

Um ponto importante no entendimento das propriedades de transporte das nanofitas de grafeno é o efeito da dopagem nesses sistemas. Em alguns trabalhos recentes [136], foi verificado que a dopagem de boro em GNR causa uma distorção local da rede, gerando um transporte distinto para cada canal de spin, além da polarização relacionada com as bordas, que estão localizados por volta de $1 \mathrm{eV}$ acima e abaixo do nível de Fermi. Essa polarização no transporte 
abriria a possibilidade da utilização dessas fitas como filtros de spin.

Neste capítulo, estudaremos as nanofitas de grafeno zigzag $(3,0)^{1}$. Primeiramente, iremos estudar o comportamento da corrente para nanofitas de grafeno pristinas com acoplamento ferromagnético entre as bordas do sistema. Iremos focar no transporte dependente do spin, onde estudaremos o comportamento da corrente quando os eletrodos estão em um alinhamento magnético paralelo (P) e anti-paralelo (AP). Em seguida, iremos estender os resultados para nanofitas dopadas com boro e nitrogênio. Finalmente, estudaremos um sistema que pode ser visto como uma junção túnel magnética, composta por nanofitas de grafeno e nanofitas de nitreto de boro hexagonal (h-BNR). Neste último caso, iremos focar em aplicações do tipo filtro de spin e elementos para dispositivos que utilizam o efeito da magnetoresistência.

\subsection{Metodologia}

Para o estudo das nanofitas de grafeno pristinas e dopadas com boro e nitrogênio, realizamos cálculos de primeiros princípios de energia total spin polarizadas baseadas na teoria do funcional da desidade [37,40], para determinar as propriedades estruturais e eletrônicas das nanofitas de grafeno $(3,0)$ zigzag pristinas e dopadas. Os cálculos foram realizados utilizando a aproximação generalizada do gradiente (GGA) para o termo de troca e correlação como proposto por Perdew et al. [91]. Para a descrição da interação entre os elétrons de caroço e valência, foram utilizados pseudo-potenciais de norma conservada, como proposto por Troullier-Martins [124]. Os cálculos das propriedades estruturais e eletrônicas foram realizados no código SIESTA. Foi utilizada uma base dupla- $\zeta$ mais uma função de polarização (DZP) com uma energia de corte de $300 R y$. As estruturas foram consideradas relaxadas quando as forças residuais nos átomos fossem menores que $0.03 \mathrm{eV} / \AA$.

As propriedades de transporte foram obtidas utilizando o formalismo de funções de Green fora do equilíbrio, conforme descrito na seção 3.2. A seguir, faremos uma breve recapitulação do formalismo de NEGF implementado no código TRANSAMPA. Isso é necessário para o desenvolvimento das modificações utilizadas para o transporte com contatos com magnetizações diferentes.

O sistema utilizado nos cálculos de transporte é dividido em três partes: eletrodo esquerdo

\footnotetext{
${ }^{1}$ Iremos utilizar no decorrer deste capítulo a notação de Ezawa para a classificação das nanofitas de grafeno [137].
} 
(LL), região central (CC) e eletrodo direito (RR). A região central, contém a região de espalhamento e duas regiões de buffer, que são regiões com características dos eletrodos pristinos, para que o acoplamento entre as partes do sistema sejam perfeitos. Esse sistema pode ser visto esquematicamente na figura 8.1 .

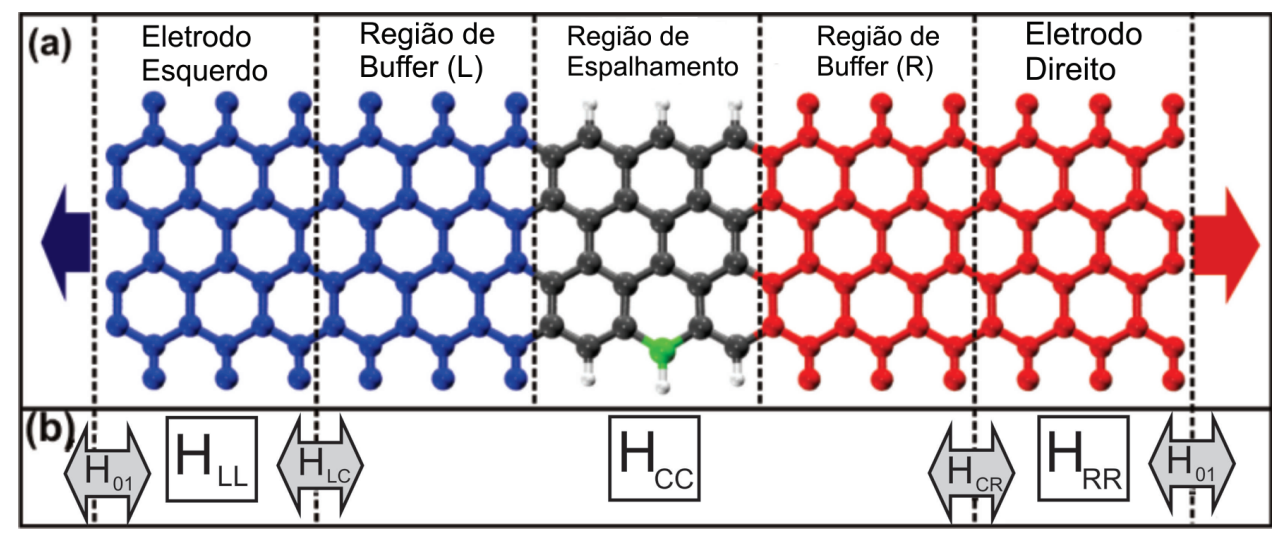

Figura 8.1: (a)Representação esquemática do sistema utilizado nos cálculos de transporte eletrônico. O dispositivo é composto pelos eletrodos direito(R) e esquerdo(L) e uma região de espalhamento (C), que é conectada aos eletrodos pelas regiões de buffer direita e esquerda. $\mathrm{O}$ átomo em verde na figura marca a configuração mais estável para os átomos de boro e nitrogênio utilizados na dopagem das nanofitas. (b) Represetação do sistema em termos das matrizes Hamiltonianas de cada bloco utilizado nos cálculos.

Uma vez as matrizes hamiltonianas (H) e de overlap (S) forem calculadas no código SIESTA, é possível escrever as equações abaixo para as funções de Green do sistema:

$$
\left.\begin{array}{ccc}
E \mathbf{S}_{L L}-\mathbf{H}_{L L} & E \mathbf{S}_{L C}-\mathbf{H}_{L C} & 0 \\
E \mathbf{S}_{C L}-\mathbf{H}_{C L} & E \mathbf{S}_{C C}-\mathbf{H}_{C C} & E \mathbf{S}_{C R}-\mathbf{H}_{C R} \\
0 & E \mathbf{S}_{R C}-\mathbf{H}_{R C} & E \mathbf{S}_{R R}-\mathbf{H}_{R R}
\end{array}\right) \times\left(\begin{array}{ccc}
\mathbf{g}_{L L} & \mathbf{g}_{L C} & \mathbf{g}_{L R} \\
\mathbf{g}_{C L} & \mathbf{g}_{C C} & \mathbf{g}_{C R} \\
\mathbf{g}_{R L} & \mathbf{g}_{R C} & \mathbf{g}_{R R}
\end{array}\right)=
$$

que pode ser resolvida, obtendo-se a função de Green para a região de espalhamento $\mathbf{g}_{C C}$,

$$
g_{C C}(E)=\left(E \mathbf{S}_{C C}-\mathbf{H}_{C C}-\Sigma_{L}(E)-\Sigma_{R}(E)\right)^{-1},
$$

onde introduzimos os termos conhecidos como "auto-energias"dos eletrodos $\Sigma_{L} / \Sigma_{R}$, que descrevem a influência dos eletrodos na estrutura eletrônica do sistema. Essas quantidades podem ser calculadas a partir das funções de Green de superfície dos eletrodos semi-infinitos isolados, 
dadas por:

$$
\begin{gathered}
\mathbf{g}_{L L}(E)=\left(E \mathbf{S}_{L L}-\mathbf{H}_{L L}\right)^{-1} \\
\mathbf{g}_{R R}(E)=\left(E \mathbf{S}_{R R}-\mathbf{H}_{R R}\right)^{-1}
\end{gathered}
$$

e as auto-energias são dadas por

$$
\begin{gathered}
\Sigma_{L}(E)=\left(E \mathbf{S}_{L C}-\mathbf{H}_{L C}\right) \mathbf{g}_{L L}(E)\left(E \mathbf{S}_{C L}-\mathbf{H}_{C L}\right) \\
\Sigma_{R}(E)=\left(E \mathbf{S}_{R C}-\mathbf{H}_{R C}\right) \mathbf{g}_{R R}(E)\left(E \mathbf{S}_{C R}-\mathbf{H}_{C R}\right)
\end{gathered}
$$

As funções de Green de superfície são obtidas utilizando-se um algoritmo recursivo como descrito em [61]. Finalmente, podemos definir as matrizes de acoplamento entre a região central e os eletrodos, que permitem o cálculo da função de transmissão, dadas por

$$
\begin{aligned}
& \Gamma_{L}(E)=i\left(\Sigma_{L}(E)-\Sigma_{L}^{\dagger}(E)\right) \\
& \Gamma_{R}(E)=i\left(\Sigma_{R}(E)-\Sigma_{R}^{\dagger}(E)\right)
\end{aligned}
$$

Assim, usando as relações descritas anteriormente, é possível calcular a transmitância do sistema para um processo balístico

$$
T(E)=\operatorname{Tr}\left[\Gamma_{L} \mathbf{g}_{C C}^{A} \Gamma_{R} \mathbf{g}_{C C}^{R}\right]
$$

Se não existir nenhuma tensão de bias aplicado ao sistema, este se encontra no regime de resposta linear, e as propriedades de transporte são todas obtidas como descrito anteriormente.

Quando um bias externo é aplicado, a densidade de carga na região de espalhamento será diferente da situação de equilíbrio. Nos eletrodos, a aplicação de um bias irá causar um shift rígido nos seus auto-valores. Entretanto, a hamiltoniana do sistema agora é escrita como:

$$
\mathbf{H}=\left(\begin{array}{ccc}
\mathbf{H}_{S S}+\mathbf{S}_{L} e V / 2 & \mathbf{H}_{L C} & \mathbf{0} \\
\mathbf{H}_{C L} & \mathbf{H}_{C C} & \mathbf{H}_{C R} \\
\mathbf{0} & \mathbf{H}_{R C} & \mathbf{H}_{S S}+\mathbf{S}_{L} e V / 2
\end{array}\right)
$$

Como a hamiltoniana do sistema depende da matriz densidade, é necessário que esta, agora, 
seja calculada de maneira auto-consistente levando-se em conta a presença do bias externo. Assim, a matriz densidade do problema pode ser calculada utilizando a função de Green Lesser do sistema $[44,138,139]$ :

$$
\rho=\frac{1}{2 \pi i} \int d E \mathbf{g}^{<}(E)
$$

onde $\mathbf{g}^{<}(E)$ é dada por:

$$
\mathbf{g}^{<}(E)=\mathbf{g}^{R}(E) \boldsymbol{\Sigma}^{<}(E) \mathbf{g}^{A}(E)
$$

e $\Sigma^{<}$é dada por

$$
\boldsymbol{\Sigma}^{<}=i\left[\Gamma_{L} f\left(E-\mu_{L}\right)+\Gamma_{R} f\left(E-\mu_{R}\right)\right]
$$

Assim, a função de Green lesser pode ser escrita como:

$$
\mathbf{g}^{<}(E)=i \mathbf{g}^{R}(E)\left[\Gamma_{L} f\left(E-\mu_{L}\right)+\Gamma_{R} f\left(E-\mu_{R}\right)\right] \mathbf{g}^{A}(E)
$$

O procedimento auto-consistente é realizado utilizando o seguinte algoritmo:

- Primeiro, calcula-se uma densidade de carga inicial - $\rho^{0}(\mathbf{r})=\left\langle\mathbf{r}\left|\rho_{C}^{0}\right| \mathbf{r}\right\rangle$ - que é utilizada para calcular os elementos da matriz hamiltoniana e de overlap;

- $\mathbf{g}_{C C}, \Gamma_{L}$ e $\Gamma_{R}$ são calculadas;

- Uma vez que as matrizes do item anterior foram calculadas, calcula-se a função de Green lesser, $\mathbf{g}^{<}$, que é integrada em todo o espectro, para a obtenção de uma nova matriz densidade;

- Este procedimento é repetido até que uma solução auto-consistente seja obtida, utilizandose um critério de convergência, $\delta$, onde $\operatorname{Max}\left|\rho^{i}-\rho^{i+1}\right|<\delta$. $\left(\delta \approx 10^{-6}\right.$.

Seguindo a fórmula de Landauer no procedimento de Meier-Wingreen [58], a corrente do sistema é obtida como:

$$
\begin{array}{r}
I=\frac{2 e^{2}}{h} \int d E T r\left[\Gamma_{L}(E, V) g_{C C}^{A}(E, V) \Gamma_{R}(E, V) g_{C C}^{R}(E, V)\right] \times \\
\left(f\left(E-\mu_{L}\right)-f\left(E-\mu_{R}\right)\right)
\end{array}
$$


onde $\mu_{L / R}=\mu \pm e V$, e $f\left(E-\mu_{L / R}\right.$ são as funções de distribuição de Fermi-Dirac para uma dada temperatura. A transmitância do sistema na presença de um bias externo é dada por:

$$
T(E, V)=\operatorname{Tr}\left[\Gamma_{L}(E, V) g_{C C}^{A}(E, V) \Gamma_{R}(E, V) g_{C C}^{R}(E, V)\right]
$$

Até agora, toda esta descrição serviu como uma recapitulação do formalismo descrito na seção 3.2. A seguir, iremos descrever as modificações realizadas para os cálculos com configurações magnéticas dos eletrodos diferentes.

\subsubsection{Transporte dependente do spin}

Para generalizar o formalismo descrito anteriormente de forma a tratarmos sistemas magnéticos, a hamiltoniana e a função de Green do sistema devem ser dependentes do spin, podendo serem escritas como:

$$
\begin{aligned}
\mathbf{H}_{C C} & =\left(\begin{array}{ll}
\mathbf{H}_{C C}^{\uparrow \uparrow} & \mathbf{H}_{C C}^{\uparrow \downarrow} \\
\mathbf{H}_{C C}^{\downarrow \uparrow} & \mathbf{H}_{C C}^{\uparrow \uparrow}
\end{array}\right) \\
\mathbf{g}_{C C} & =\left(\begin{array}{ll}
\mathbf{g}_{C C}^{\uparrow \uparrow} & \mathbf{g}_{C C}^{\uparrow \downarrow} \\
\mathbf{g}_{C C}^{\downarrow \uparrow} & \mathbf{g}_{C C}^{\downarrow \downarrow}
\end{array}\right)
\end{aligned}
$$

Assumindo que não exista nenhum mecanismo que misture as componentes de spin, os termos $\uparrow \downarrow$ e $\downarrow \uparrow$ são nulas. Assim, as auto-energias do sistema e matrizes de acoplamento são escritas como:

$$
\begin{gathered}
\boldsymbol{\Sigma}_{\mathbf{L} / \mathbf{R}}(E)=\left(\begin{array}{cc}
\boldsymbol{\Sigma}_{L / R}^{\uparrow} & 0 \\
0 & \boldsymbol{\Sigma}_{L / R}^{\downarrow}
\end{array}\right) \\
\boldsymbol{\Gamma}_{\mathbf{L} / \mathbf{R}}(E)=\left(\begin{array}{cc}
\boldsymbol{\Gamma}_{L / R}^{\uparrow} & 0 \\
0 & \Gamma_{L / R}^{\downarrow}
\end{array}\right)
\end{gathered}
$$

De posse dessas matrizes, a transmitância pode ser escrita em uma forma dependente do spin como:

$$
T^{\sigma_{1} \sigma_{2}}(E)=\operatorname{Tr}\left[\Gamma_{L}^{\sigma_{1}}(E) g_{C C}^{A}(E)^{\sigma_{1} \sigma_{2}} \Gamma_{R}^{\sigma_{2}}(E) g_{C C}^{R}(E)^{\sigma_{1} \sigma_{2}}\right]
$$

onde $\sigma_{1}=\sigma_{2}=\uparrow, \downarrow$. 


\subsection{Resultados e Discussões}

\subsection{Nanofita de grafeno $(3,0)$ pristina}

Inicialmente, consideramos o transporte de carga através de uma nanofita de grafeno $(3,0)$ pristina. As nanofitas de grafeno zigzag apresentam uma polarização de spin nas bordas, com um acoplamento ferromagnético (FM) ao longo da direção de crescimento da fita. Entre as bordas, esse acoplamento pode tanto ser ferromagnético (FM) como anti-ferromagnético (AFM). Iremos considerar somente o acoplamento ferromagnético entre as bordas, o que leva o sistema a possuir um caráter metálico. Para os eletrodos, estudamos duas possíveis configurações: (i) os eletrodos possuem o mesmo alinhamento de spin, que iremos chamar de configuração paralela (P) ou ferromagnética (FM), veja figura 8.2(a); (ii) os eletrodos esquerdo e direito possuem um alinhamento anti-paralelo(AP) ou configuração AFM, veja figura 8.2(b).
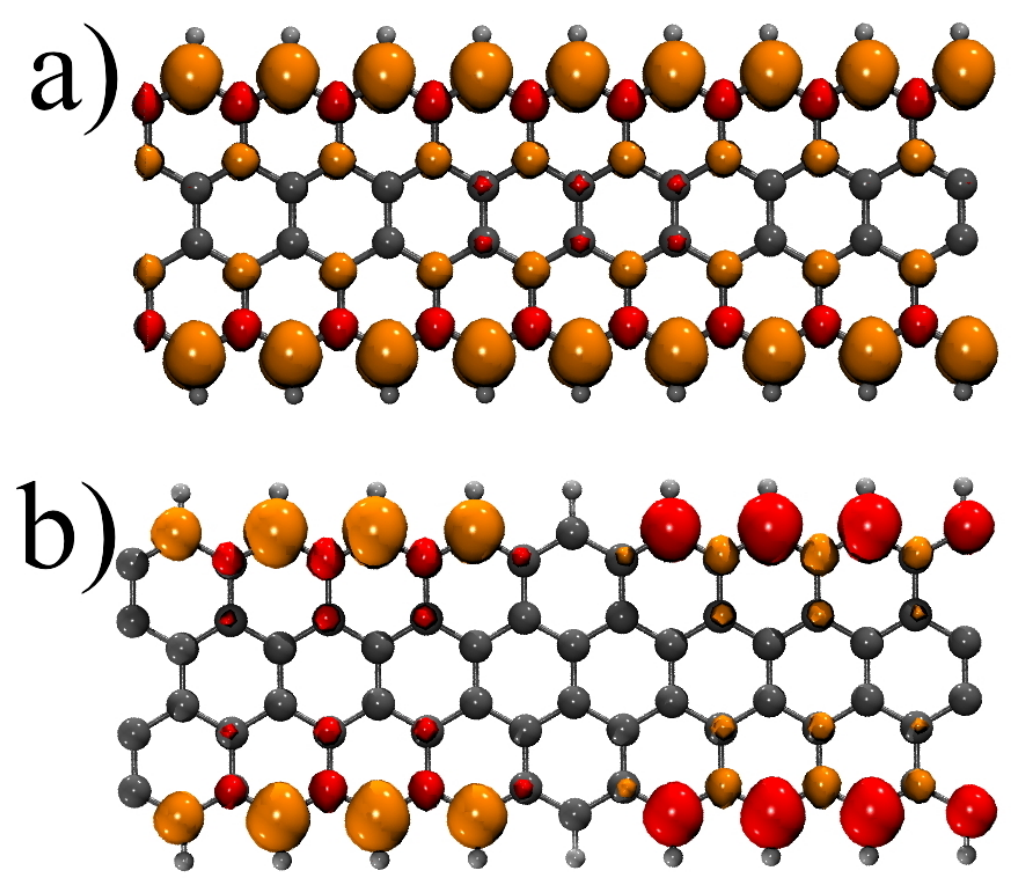

Figura 8.2: Diferença local entre as densidades de carga para os spins up e down, $\rho_{\text {up }}-\rho_{\text {down }}$, para a nanofita de grafeno $(3,0)$ nas configurações: (a) Ferromagnética (FM) e (b) Antiferromagnética (AFM). As cores laranja e vermelha correspondem a spin up e down, respectivamente. $\mathrm{O}$ valor da isosuperfície utilizada foi de $0.002 e / b o h r^{3}$.

Um plot na densidade de magnetização $\left(\rho_{u p}-\rho_{\text {down }}\right)$ para as configurações FM e AFM dos eletrodos é mostrado na figura 8.2(a) e (b), respectivamente. Quando as magnetizações das bordas dos dois eletrodos estão com o mesmo alinhamento, temos um comportamento ferro- 
magnético em todo o sistema, figura 8.2(a). Por outro lado, para um alinhamento antiparalelo entre os dois eletrodos, temos um comportamento antiferromagnético, como pode ser visto na figura 8.2(b). Na configuração AFM, a magnetização total vai a zero na região central do sistema. Isso ocorre devido a um overlap destrutivo entre os spins.

a)

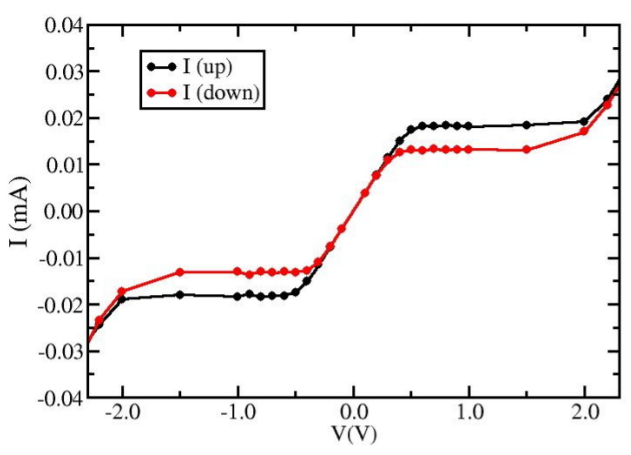

c)

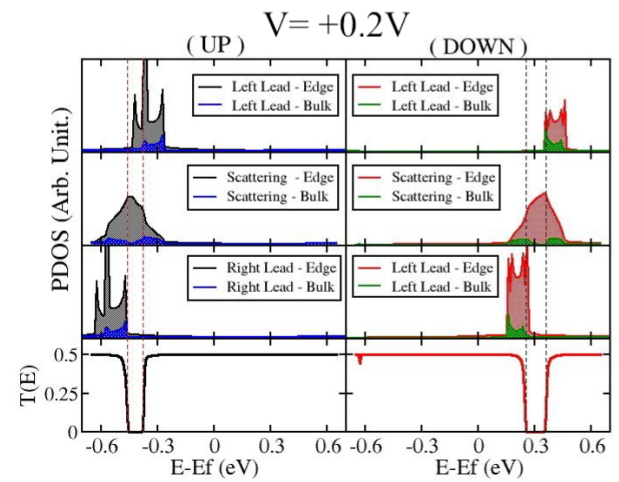

b)

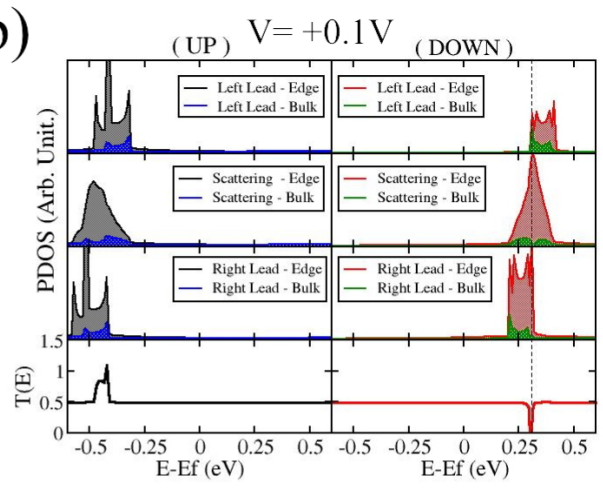

d)

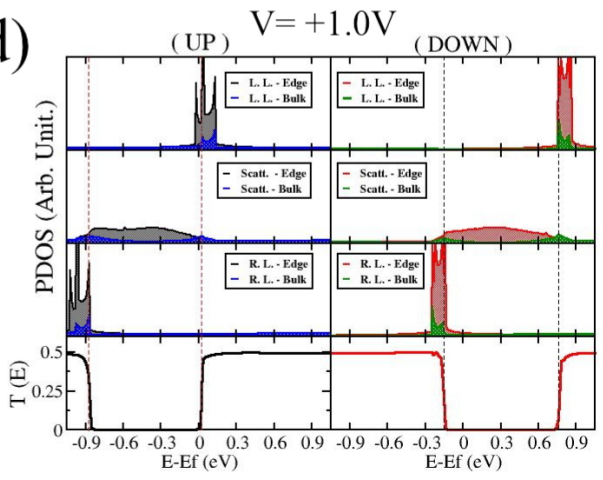

Figura 8.3: (a) Curvas $I \times V$ dependentes do spin para uma nanofita de grafeno $(3,0)$ com alinhamento FM dos eletrodos. A curva preta e vermelha correspondem aos spins up e down, respectivamente. (b), (c) e (d) Densidade de estados projetada nos átomos de carbono das bordas e na região de bulk das nanofitas $\mathrm{e}$, curvas de transmitância para diferentes voltages de bias: $+0.1,+0.2 \mathrm{e}+1.0 \mathrm{~V}$, respectivamente.

Na figura 8.3(a), apresentamos as curvas $I \times V$ para a configuração FM. Podemos observar que para voltagens entre $\pm 0.3 \mathrm{~V}$ a corrente para os spins up e down são degeneradas. Acima e abaixo desse intervalo, a corrente para spins up e down são desdobradas uma da outra, onde a corrente para o canal up é sempre maior que para o down. Para entender isso, mostramos na figura 8.3(b), (c) e (d) a evolução da densidade de estados projetada (PDOS) do sistema na presença do bias aplicado. Quando o sistema não está sujeito a uma tensão de bias, os estados de borda nos eletrodos esquerdo e direito, estão perfeitamente alinhados com os estados da região de espalhamento. Esse casamento construtivo entre os estados leva a uma transmissão perfeita. Se um bias é aplicado ao sistema, este acoplamento entre os estados é quebrado, 
diminuindo a probabilidade de transmissão na região.

Para entender porque o desdobramento ocorre entre os canais up e down, na corrente do sistema entre $\pm 0.5 \mathrm{~V}$ e $\pm 2.0 \mathrm{~V}$, primeiramente lembramos que a corrente é dada pela equação 8.15, onde a transmitância do sistema e a função de Fermi-Dirac dos eletrodos esquerdo e direito possuem um papel central. Na figura 8.4(a), apresentamos as transmitâncias up e down e as funções de Fermi-Dirac dos eletrodos, para um bias aplicado de $+1.0 \mathrm{~V}$. Analisando o gráfico para a componente up do spin, verificamos que acima e abaixo das linhas verticais, regiões (i) e (v), a diferença entre as funções de Fermi-Dirac é zero. Na região (ii), a transmitância do sistema é zero. Assim, as regiões (i), (ii) e (v) não contribuem para a corrente. Nas regiões (iii) e (iv), a transmitância a diferença das funções de FD são diferentes de zero, contribuindo para a corrente. Esta mesma análise pode ser feita para o canal down do spin, onde é observado o mesmo comportamento. Ainda na figura 8.4(a) painel inferior, apresentamos o produto da transmitância com a diferença das funções de Fermi-Dirac ${ }^{2}$. Assim, olhando para essa quantidade, observamos que a contribuição para o canal up é maior que para o down. Essa diferença é responsável pelo desdobramento observado na corrente. Para confirmar essa análise, na figura 8.4(b), mostramos os mesmos resultados da análise anterior para $+0.2 \mathrm{~V}$, onde as correntes são degeneradas. Podemos observar, agora, que a contribuição para a corrente é a mesma para ambos os canais de spin, levando a corrente ser degenerada no spin, como apresentado na figura 8.3(a).

Na figura 8.5(a), mostramos as curvas $I \times V$ para a configuração AFM dos eletrodos. Neste caso, observamos um comportamento de um filtro de spin, onde para bias positivos temos somente corrente up e para bias negativo, temos somente corrente down. Como era de se esperar, esse comportamento é simétrico ao redor da voltagem de bias nula.

Este comportamento de filtro de spin pode ser explicado, observando como a transmitância do sistema varia em função da tensão de bias aplicada, figura 8.6. Para voltagem de bias zero, verificamos que existe um gap de transmitância idêntico, para ambos os canais de spin ao redor do nível de Fermi. Entretanto, para um bias positivo, esse gap de transmitância diminui para o canal up, enquanto que para o down ele aumenta, levando ao comportamento de filtro de spin observado. De maneira similar, o mesmo comportamento ocorre para bias negativo, mas com os papéis invertidos para os canais de spin.

\footnotetext{
${ }^{2}$ Este produto integrado na janela de energia é que fornece a corrente, a menos de uma constante.
} 


\section{(a)}

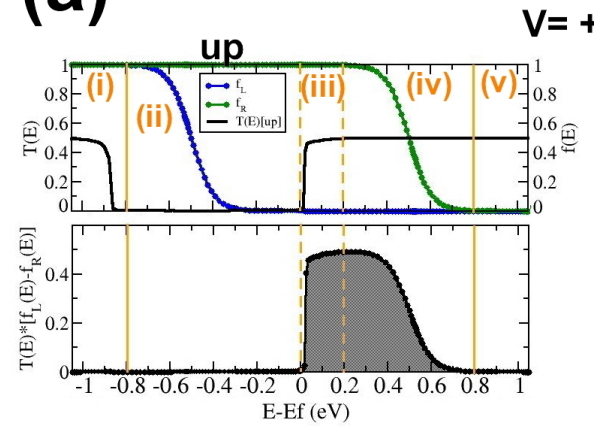

$$
V=+1.0 \mathrm{~V}
$$
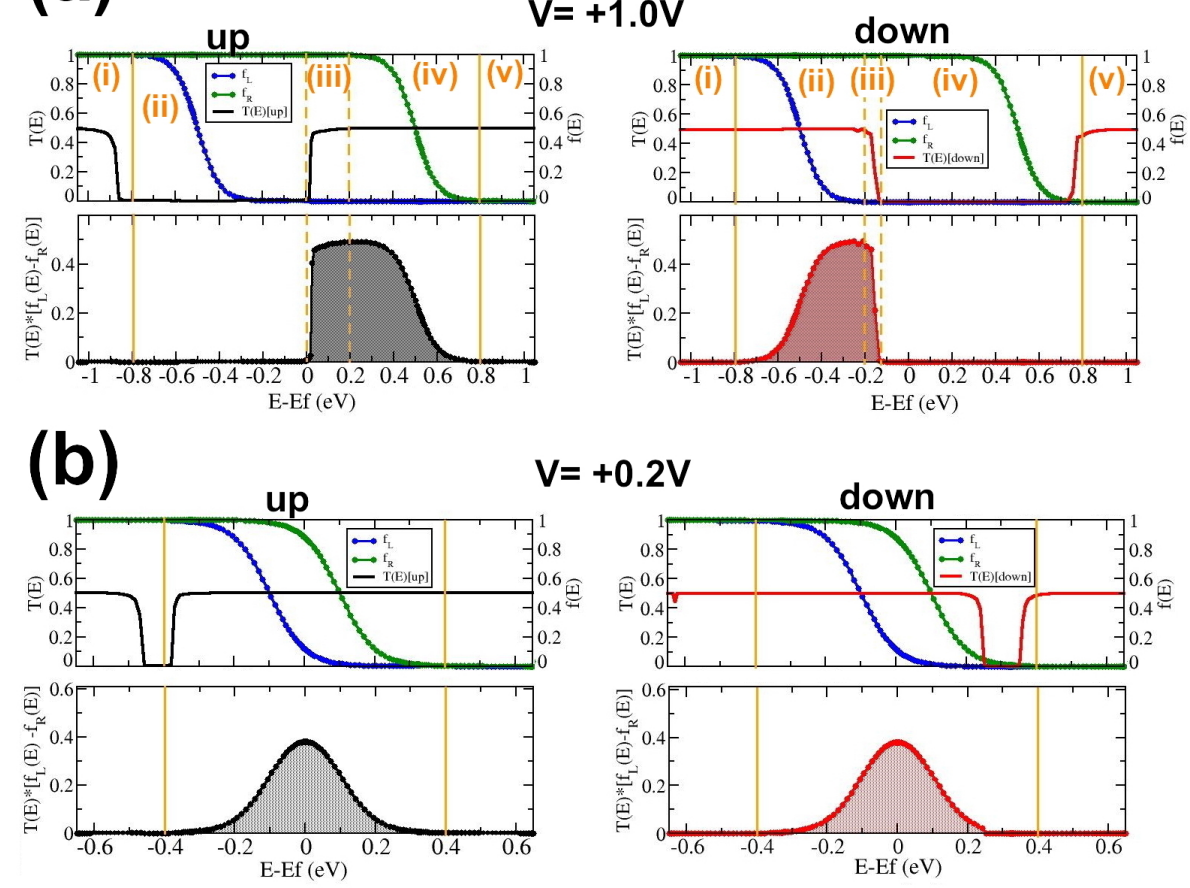

Figura 8.4: (a) Painel superior: Transmitância e função de distribuição de Fermi-Dirac em função da energia para os spins up e down. Painel inferior: Produto da transmitância com a diferença das funções de Fermi-Dirac para os eletrodos esquerdo e direto, para uma voltagem de bias de $+1.0 \mathrm{~V}$. (b) Painel superior: Transmitância e função de distribuição de Fermi-Dirac em função da energia para os spins up e down. Painel inferior: Produto da transmitância com a diferença das funções de Fermi-Dirac para os eletrodos esquerdo e direto, para uma voltagem de bias de $+0.2 \mathrm{~V}$.

\subsection{Dopagem da nanofita com boro e nitrogênio}

Os efeitos da dopagem das nanofitas de grafeno zigzag com boro e nitrogênio tem sido amplamente discutidos na literatura [135,136,140-142]. A configuração energeticamente mais estável é quando os átomos de boro e nitrogênio substituem um átomo de carbono nas bordas da nanofita, como mostrado na figura 8.7. Na tabela 8.1, mostramos as propriedades estruturais destes sistemas.

Baseados nos resultados da tabela 8.1, verificamos que a dopagem substitucional de boro e nitrogênio nas nanofitas, causam somente uma distorção local da rede da nanofita. Estes resultados estão em concordância com trabalhos anteriores [99]. Na figura 8.8, apresentamos a densidade de magnetização de spin, $\rho_{u p}-\rho_{\text {down }}$, para as dopagens de boro(a) e nitrogênio(b). Quando estes átomos estão dopando uma nanofita ferromagnética, eles geram uma supressão da magnetização local na borda na região onde se encontra a impureza, enquanto que a outra borda fica inalterada. 

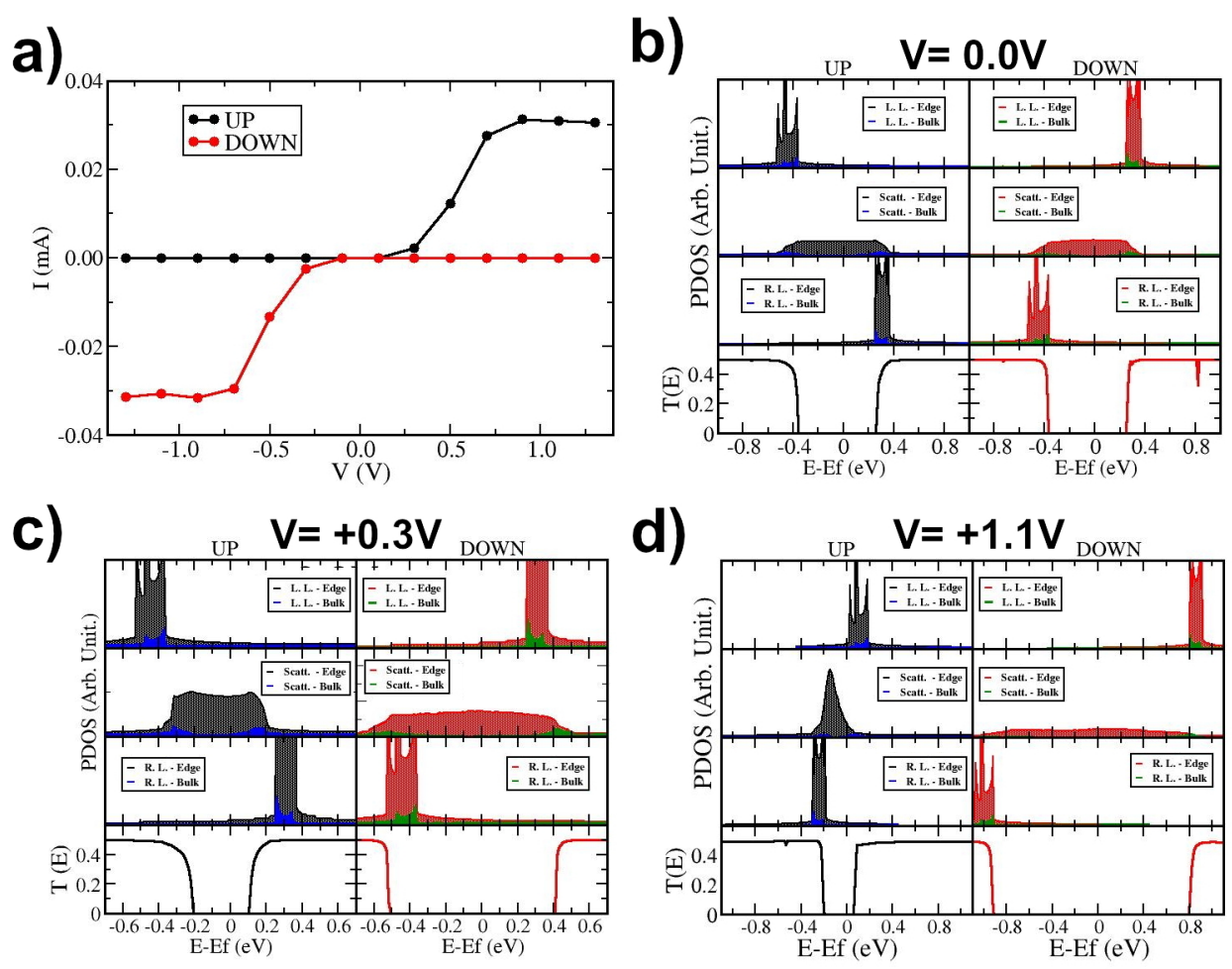

Figura 8.5: (a) Curvas $I \times V$ dependentes do spin para a nanofita de grafeno na configuração dos eletrodos AFM. As curvas em preto e azul são para as correntes up e down, respectivamente. Densidade de estados projetada nos átomos de carbono de borda e bulk, e transmitâncias para diferentes voltagens de bias: (b) $0.0 \mathrm{~V}$; (c) $+0.3 \mathrm{~V}$; e (d) $+1.1 \mathrm{~V}$.

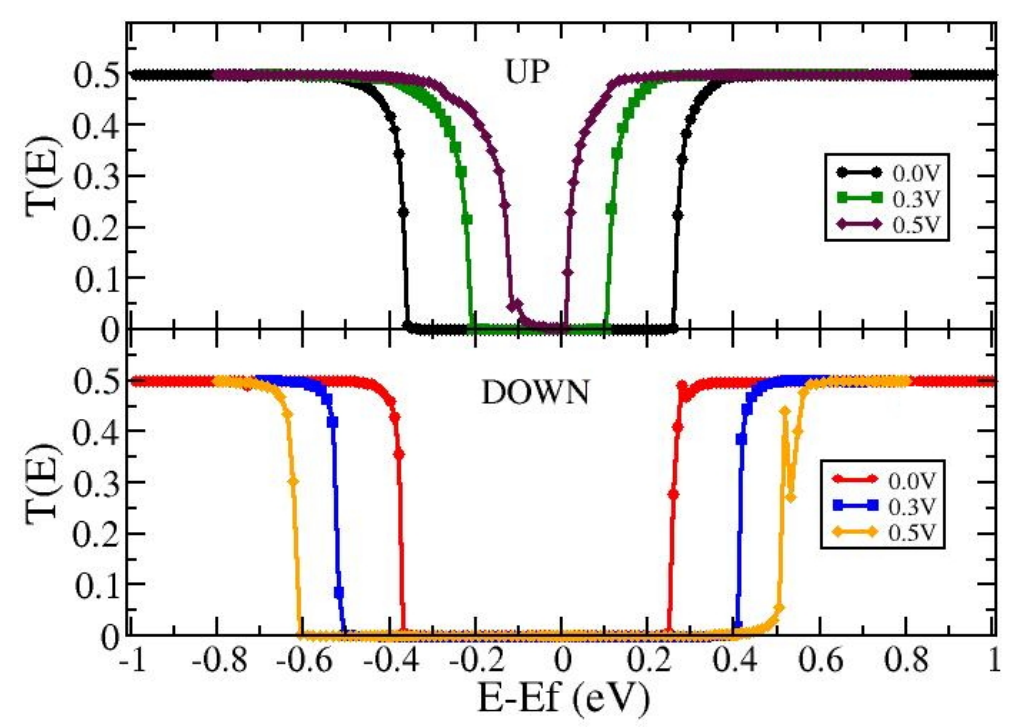

Figura 8.6: Transmitâncias para os canais de spin up e down, em função da voltagem de bias para a configuração AFM dos eletrodos.

Para as nanofitas dopadas com boro e nitrogênio, calculamos as curvas $I \times V$, com os eletrodos sempre na configuração ferromagnética. Na figura 8.9, apresentamos os resultados 

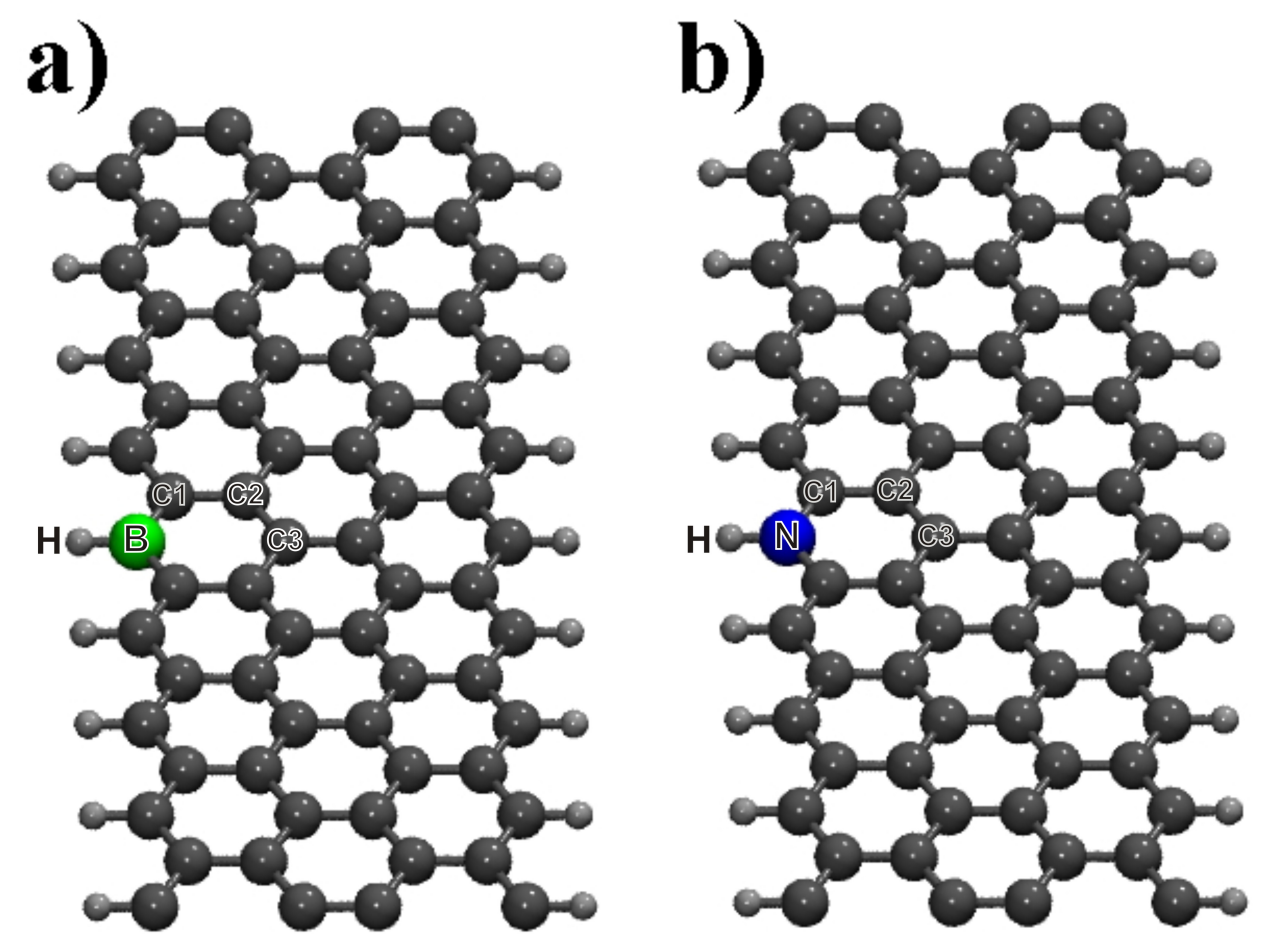

Figura 8.7: Representação esquemática das nanofitas dopadas com boro e nitrogênio.

Tabela 8.1: Propriedades estruturais das nanofitas de grafeno dopadas com boro e nitrogênio

\begin{tabular}{c|c|c}
\hline \hline Dopante & Ligação & Comprimento da ligação $(\AA)$ \\
\hline \hline \multirow{3}{*}{$\mathrm{B}$} & $\mathrm{B}-\mathrm{H}$ & 1.23 \\
& $\mathrm{~B}-\mathrm{C} 1$ & 1.53 \\
& $\mathrm{C} 1-\mathrm{C} 2$ & 1.46 \\
& $\mathrm{C} 2-\mathrm{C} 3$ & 1.45 \\
\hline \multirow{3}{*}{$\mathrm{N}$} & $\mathrm{N}-\mathrm{H}$ & 1.02 \\
& $\mathrm{~N}-\mathrm{C} 1$ & 1.39 \\
& $\mathrm{C} 1-\mathrm{C} 2$ & 1.45 \\
& $\mathrm{C} 2-\mathrm{C} 3$ & 1.44 \\
\hline \hline
\end{tabular}

para a dopagem de boro. Observamos que esse sistema apresenta uma pequena diferença entre as correntes up e down, entretanto, ele ainda pode ser utilizado como um filtro de spin. Além disso, existe uma inversão entre as correntes up e down ao redor de $\pm 1.0 \mathrm{~V}$.

Para entender este comportamento, a figura 8.9(b), (c) e (d) apresenta as transmitâncias e as densidades de estados projetadas (PDOS) nos átomos de carbono das bordas e bulk para três regiões do sistema - eletrodo esquerdo, região de espalhamento e eletrodo direito - e também para o átomo de boro. Na figura 8.9(b), podemos observar que os estados do boro, para spin down, são mais localizados que os para o spin up [136]. Como consequiência, em torno do 

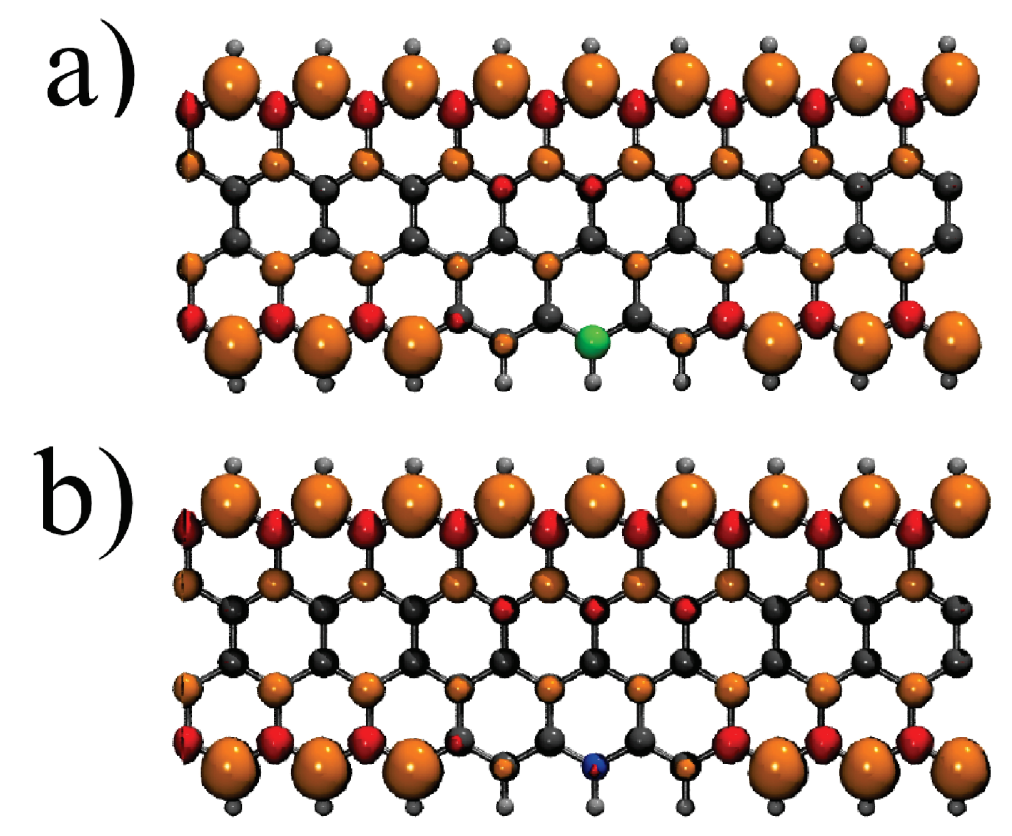

Figura 8.8: Diferença local entre as densidades de carga para os spins up e down, $\rho_{\text {up }}-\rho_{\text {down }}$, para a nanofita de grafeno $(3,0)$ dopadas com (a) boro e (b) nitrogênio. As cores laranja e vermelha correspondem a spin up e down, respectivamente. $\mathrm{O}$ valor utilizado para a isosuperfície de $0.002 e / b o h r^{3}$.

nível de Fermi, os spins up serão mais espalhados que os down. Assim, a transmitância para o canal down será maior que para o up. A polarização de spin calculada no nível de Fermi foi de $-0.16^{3}$.

Como pode ser visto na figura 8.9(c), esse comportamento permanece válido para bias abaixo de aproximadamente $+1.0 \mathrm{~V}$, com os estados down do boro sendo mais localizados que os up, tal que a transmitância para o canal down fica sempre maior que para o up. Conseqüentemente, como pode ser visto na figura 8.9(a), para um bias de $+0.5 \mathrm{~V}$, a corrente down é maior que para o canal up. Aumentando o bias até $+1.5 \mathrm{~V}$, observamos uma inversão desse comportamento, sendo agora os estados up mais localizados e por conseqüência o espalhamento no canal down é maior, fazendo com que a corrente up fique maior. O mesmo tipo análise pode ser utilizado para explicar o comportamento para tensões negativas.

Para a dopagem de nitrogênio na nanofita $(3,0)$ de grafeno zigzag, um elétron é transferido do nitrogênio para o sistema, ou seja, os estados localizados do nitrogênio estão agora abaixo do nível de Fermi. Na figura 8.10, apresentamos as curvas $I \times V$, as densidades de estados projetadas e transmitâncias para diferentes voltagens para o sistema dopado com nitrogênio.

\footnotetext{
${ }^{3}$ A polarização neste sistema é definida como: $P=\frac{T_{\uparrow}-T_{\downarrow}}{T_{\uparrow}+T_{\downarrow}}$, onde $T^{\uparrow}$ e $T^{\downarrow}$ são as transmitâncias no nível de Fermi para os canais up e down respectivamente.
} 
a)

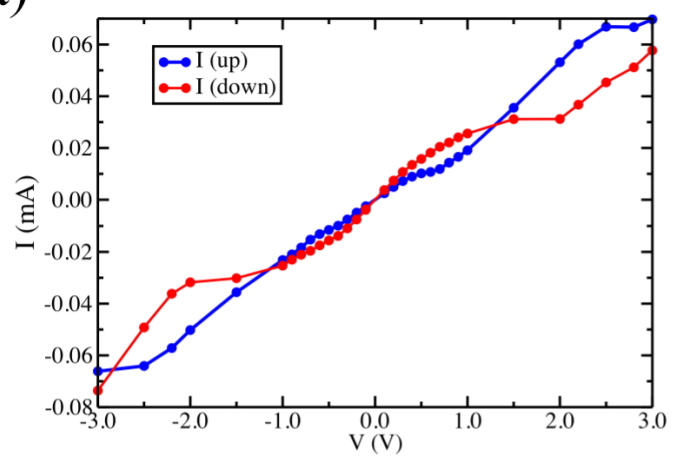

c)

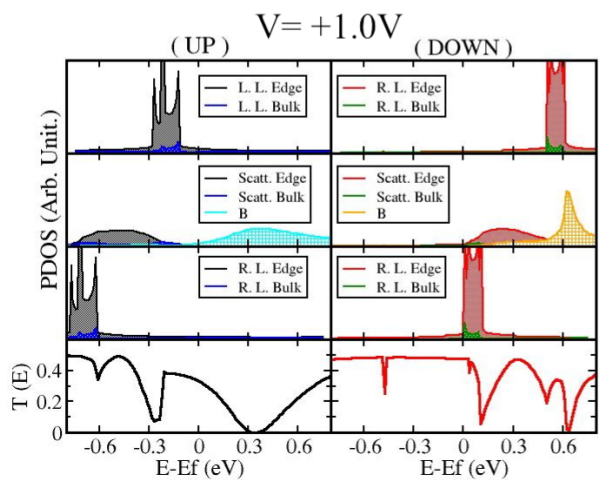

b)

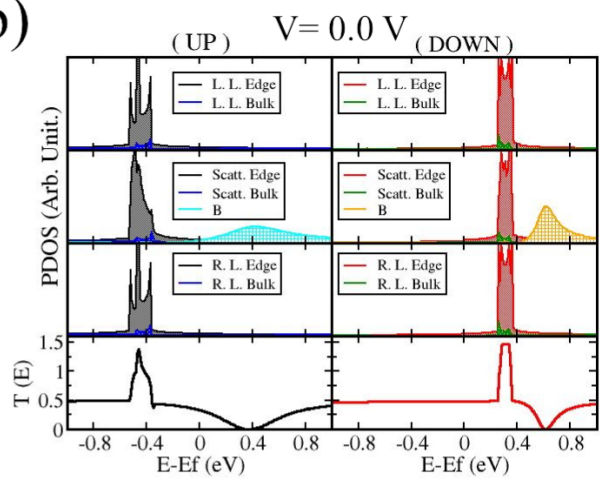

d)

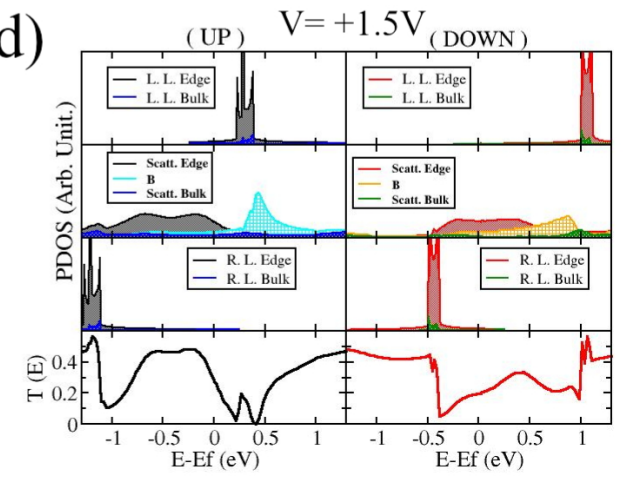

Figura 8.9: (a) Curvas $I \times V$ dependentes do spin para a nanofita de grafeno dopada com boro. As curvas em azul e vermelho são para as correntes com spin up e down, respectivamente. Densidade de estados projetada e curvas de transmitância para diferentes voltagens de bias: (b) $0.0 \mathrm{~V}$; (c) $+1.0 \mathrm{~V}$; e (d) $+1.5 \mathrm{~V}$.

Para zero bias, a polarização na transmitância calculada no nível de Fermi foi de 0.08 , sendo o canal up maior que o down. Como estamos tratando com uma impureza que doa um elétron ao sistema, o estados da impureza com spin up irão mostrar uma dispersão menor que para os spin down [140]. Essa característica pode ser verificada na PDOS nos átomos de nitrogênio, figura 8.10(b). Quando uma voltagem de bias de $+0.5 \mathrm{~V}$ é aplicada ao sistema, verificamos que a transmitância up ainda é maior que a down, levando a uma corrente maior para os spins up que os down. Entretanto, quando um bias de $+1.0 \mathrm{~V}$ é aplicado, ocorre uma inversão na corrente; a corrente down fica maior que a up. A mesma linha de raciocínio do sistema dopado com boro é valida para este sistema, ou seja, o que ocorre é uma combinação de uma variedade de processos de espalhamento e interferências relacionados com os estados de borda do sistema e com os estados localizados na impureza de nitrogênio. 
a)

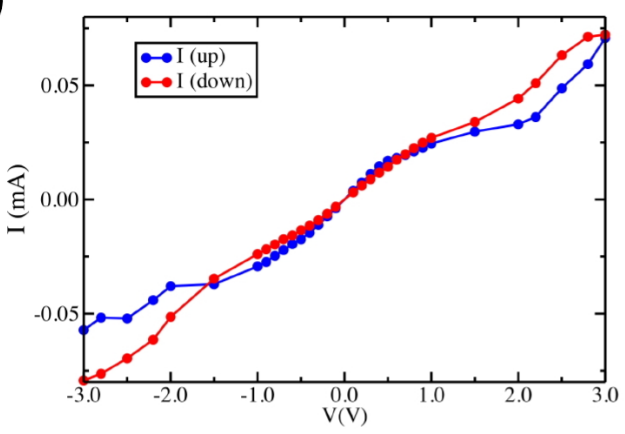

c)

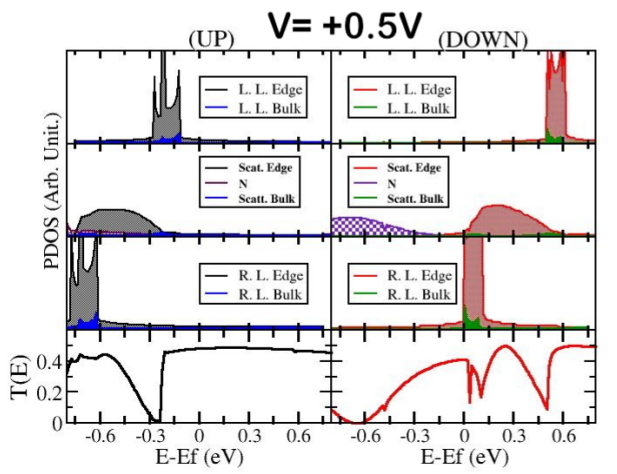

b)

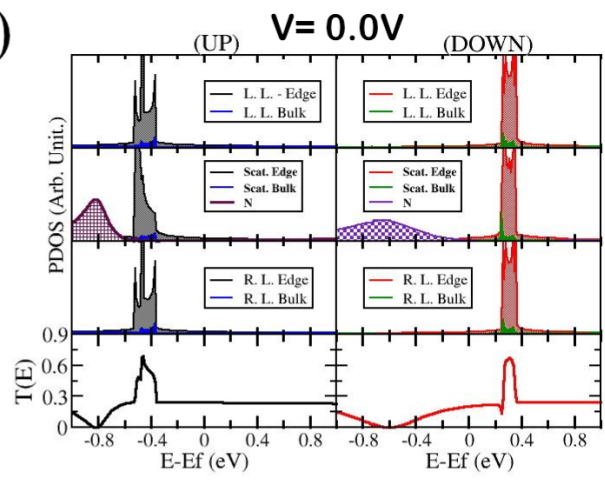

d)

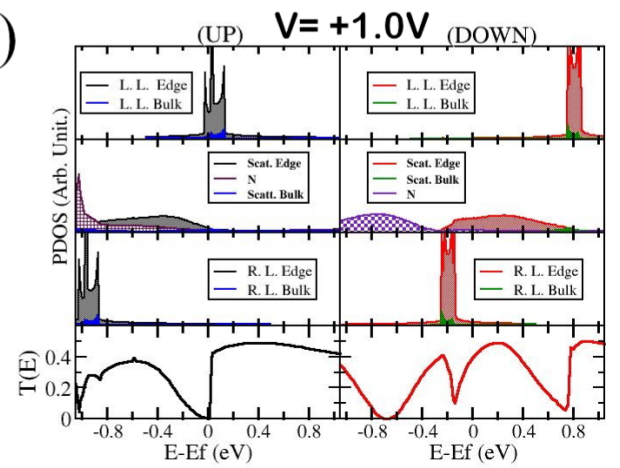

Figura 8.10: (a) Curvas $I \times V$ dependentes do spin para a nanofita de grafeno dopada com nitrogênio. As curvas em azul e vermelho são para as correntes com spin up e down, respectivamente. Densidade de estados projetada e curvas de transmitância para diferentes voltagens de bias: (b) $0.0 \mathrm{~V}$; (c) $+0.5 \mathrm{~V}$; e (d) $+1.5 \mathrm{~V}$.

\subsection{Junção túnel magnética}

Junções túnel magnéticas não são somente um dos candidatos mais promissores para aplicações em dispositivos de memórias, mas também combinam propriedades de transporte e fenômenos magnéticos de uma maneira desafiadora. Esses sistemas apresentam os fenômenos de tunelamento em uma junção de um material isolante entre dois contatos ferromagnéticos. Desta forma, podemos utilizar as nanofitas de grafeno como sendo os contatos ferromagnéticos e uma nanofita de nitreto de boro hexagonal como sendo o isolante.

Na figura 8.11(a), mostramos esquematicamente os sistemas estudados. Eles consistem de uma nanofita de grafeno $(3,0)$, onde intercalamos $N(N=1,2, \cdots, 6$, ) células unitárias de uma nanofita de $h-B N$. Na figura 8.11(b), apresentamos esquematicamente as possíveis configurações das magnetizações dos eletrodos utilizadas nos cálculos de transporte.

Na figura 8.12, mostramos um plot da densidade de magnetização, $\left(\rho_{u p}-\rho_{\text {down }}\right)$, para 3 

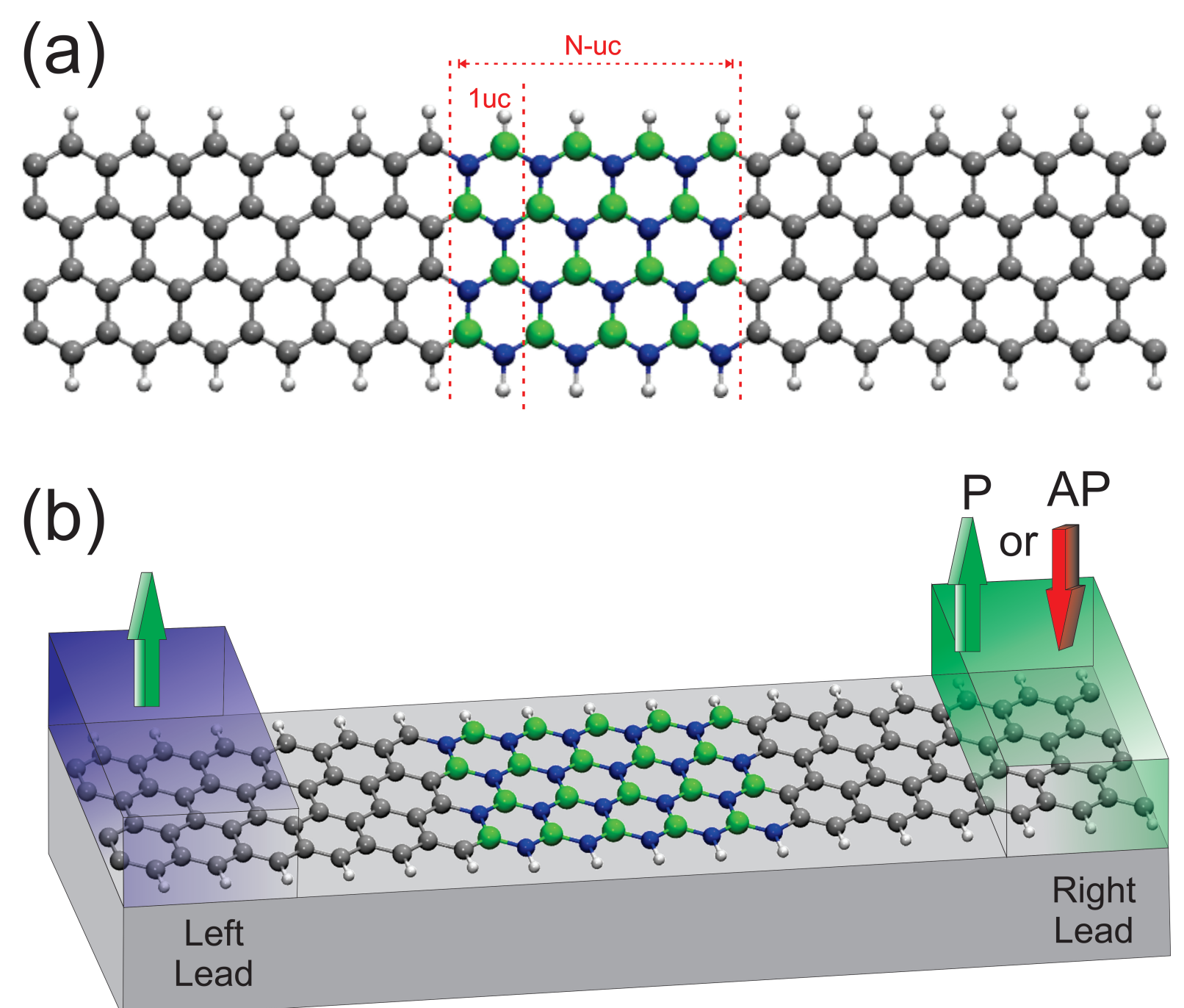

Figura 8.11: (a)Representação esquemática da geometria utilizada nos cálculos de transporte eletrônico. Os sistemas utilizados nos cálculos de transporte correspondem a $N$ uc (célula unitária), $\operatorname{com} N(N=$ $1,2, \cdots, 6)$. (b) Representação esquemática da configuração magnética dos eletrodos. Os cálculos foram realizados com um alinhamento paralelo (P) e antiparalelo (AP) dos eletrodos.

células unitárias de BN com as configurações paralela (P) e antiparalela (AP) dos eletrodos ${ }^{4}$. Primeiramente, podemos observar que as regiões compostas somente das nanofitas de grafeno estão em um acoplamento ferromagnético entre as bordas. Nesta configuração, as nanofitas são metálicas. Quando aproximamos para regiões próximas ao material isolante, ao contrário da dopagem de uma única impureza de boro ou nitrogênio, não é observada uma supressão da densidade de magnetização no sistema de grafeno vizinho ao h-BN. Um outro ponto, é que em nenhum dos casos estudados, o sistema de nitreto de boro apresenta magnetização na borda.

Dados os sistemas anteriores de 1 até 6 uc de BN, realizamos primeiramente cálculos de

\footnotetext{
${ }^{4}$ Escolhemos esses sistemas como representativos para todos os outros resultados.
} 

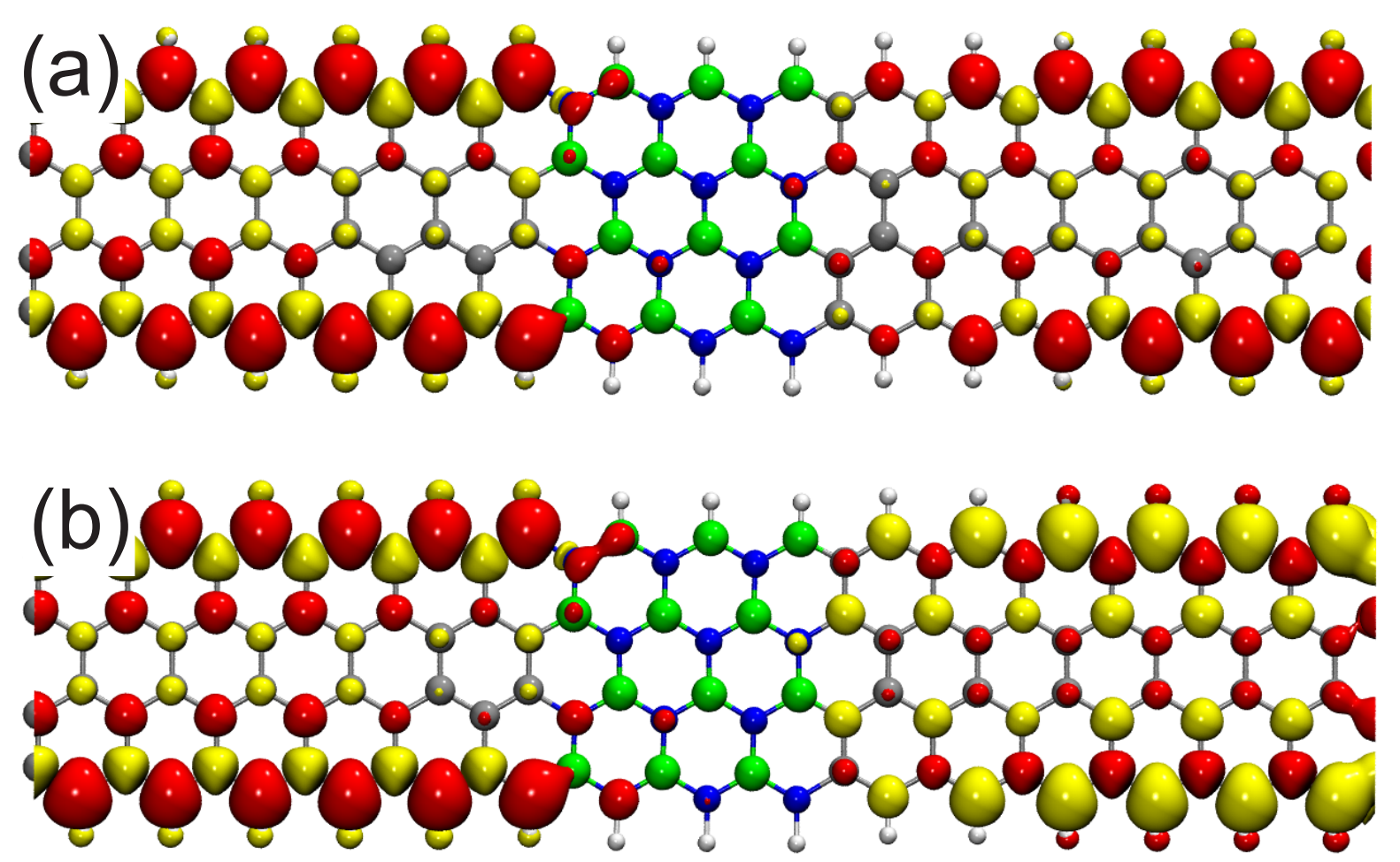

Figura 8.12: Diferença local entre as densidades de carga para os spins up e down, $\rho_{\text {up }}-\rho_{\text {down }}$, para a nanofita de grafeno $(3,0)$ para os sistemas com: (a) 3 uc de $B N$ com alinhamento paralelo dos eletrodos; (b) 3 uc de $B N$ com alinhamento antiparalelo dos eletrodos. As cores vermelha e amarela correspondem à spin up e down, respectivamente. O valor utilizado para a isosuperfície foi de $0.001 e / b o h r^{3}$.

transporte eletrônico sem a presença de um potencial de bias, para entender a influência da adição de camadas do material isolante na transmitância do sistema. Na figura 8.13, apresentamos as transmitâncias para os sistemas que consistem de 1 até 6 células unitárias de h-BN com alinhamento paralelo entre os eletrodos.

Na figura 8.13(a), apresentamos a transmitância para o sistema pristino e para o sistema com 1 célula unitária de h-BN. Observamos que a adição de uma célula unitária de BN gera uma queda no valor da transmitância em todo o intervalo de energia, apresentado na figura 8.13. Além disso, ocorre uma grande polarização dependente do spin nos estados de bulk do sistema, que ficam localizados entre $\pm 0.4 \mathrm{~V}$ ao redor do nível de Fermi. Vale ressaltar que, no caso pristino, neste intervalo de energia $( \pm 0.4 \mathrm{eV})$ o transporte é degenerado com relação ao spin. Quando aumentamos o número de células unitárias de h-BN, figuras 8.13(b,c,d,e,f) observamos claramente uma queda considerável no valor da transmitância. Entretanto, podemos observar que mesmo para 6 camadas de BN a probabilidade de transmissão eletrônica nunca vai para zero, como podemos ver nos insets em cada figura. Isso é uma clara evidência de tunela- 

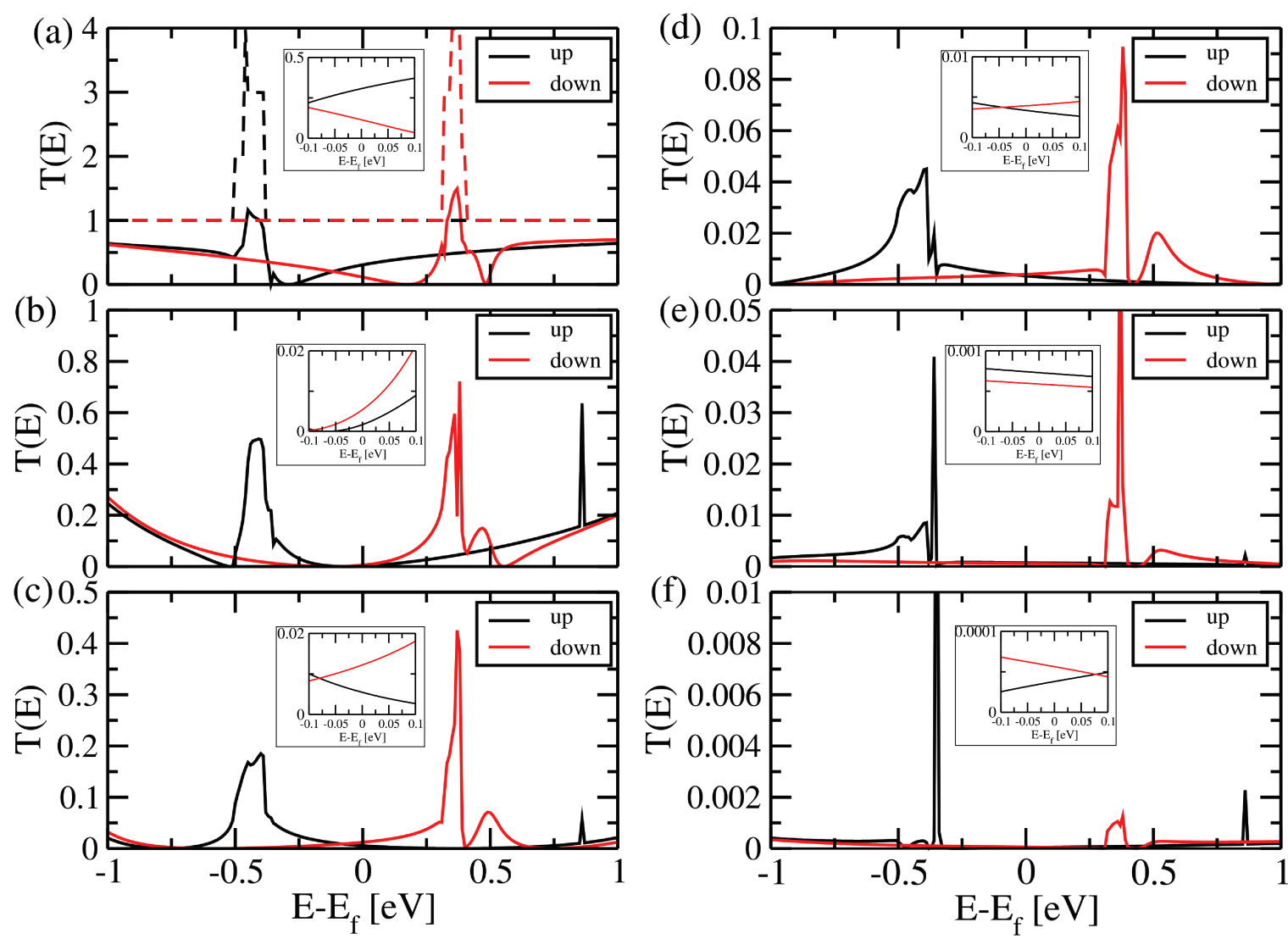

Figura 8.13: Transmitâncias para os sistemas considerados. (a)Curvas tracejadas correspondem ao sistema pristino e as curvas cheias ao sistema com 1uc de BN; (b) 2 uc de BN; (c) 3 uc de BN; (d) 4 uc de BN; (e) 5 uc de BN; (f) 6 uc de BN. Em cada gráfico apresentamos um inset, ampliando uma pequena região ao redor do nível de Fermi, para evidenciar o valor finito da transmitância.

mento através do sistema isolante. Algo importante a notar, é que em todos os casos, existe uma polarização dependente do spin para a transmitância. Baseados nos resultados apresentados, na figura 8.13 e, observando que existe uma polarização da transmitância nos sistemas mesmo utilizando uma grande quantidade de camadas isolante, por exemplo 6 células unitárias, realizamos cálculos de transporte aplicando uma tensão de bias.

Antes de entrarmos nos resultados para a junção túnel com BN, apresentamos na figura 8.14, os resultados da curva $I \times V_{d s}$ e da polarização da corrente $\left(P[\%]=\left(I_{u p}-I_{\text {down }}\right) /\left(I_{u p}+\right.\right.$ $\left.I_{\text {down }}\right)$ ) para a nanofita de grafeno pristina. Como podemos observar, somente acima de $0.3 \mathrm{~V}$ de bias é que começa a ocorrer uma polarização na corrente do sistema, as correntes up e down deixam de ser degeneradas. Como já estudamos na seção 8.3, isso ocorre devido ao desalinhamento dos estados de borda das nanofitas. Entretanto, como queremos estudar a influência da adição de BN na polarização do sistema, ou seja, o efeito que o isolante não magnético causa, 
iremos restringir os cálculos a tensões de bias menores ou igual a $0.3 \mathrm{~V}$, para evitar os efeitos gerados pelo desalinhamento dos estados de borda.
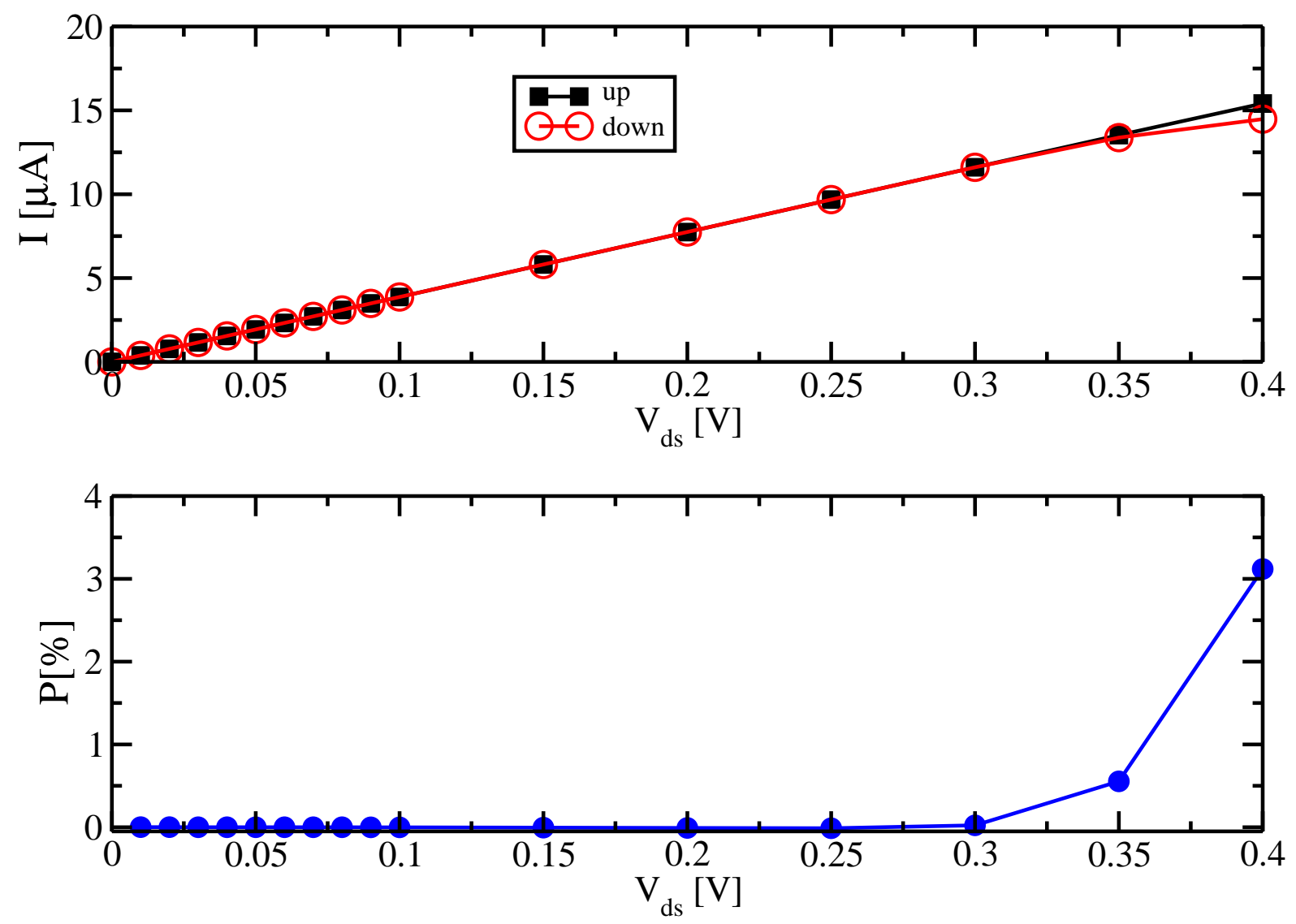

Figura 8.14: (a) Corrente em função da voltagem de bias para a nanofita de grafeno pristina com alinhamento paralelo dos eletrodos. (b) Polarização da corrente em função da voltagem de bias para a nanofita pristina.

Na figura 8.15, apresentamos as curvas de corrente em função da tensão de bias para todos os sistemas, considerando o alinhamento paralelo dos eletrodos. Observamos que o comportamento da curva em função do bias é muito semelhante em todos os casos apresentados. Observamos também nesses resultados que em alguns sistemas a corrente up é maior e em outros casos a corrente down é maior, porém não observamos um padrão para essa polarização. Além disso, um ponto importante é que mesmo a corrente caindo algumas ordens de grandeza com o aumento do número de camadas de nitreto de boro, indo de $\mu A$ até $n A$, sempre existe uma polarização da corrente.

Quando um sistema é utilizado como filtros de spin, uma grandeza muito importante é a chamada eficiência de filtragem de $\operatorname{spin}^{5}$. Esta grandeza está ligada diretamente com a polari-

\footnotetext{
${ }^{5}$ Comumente chamada de spin filter efficiency(eficiência do filtro de spin).
} 

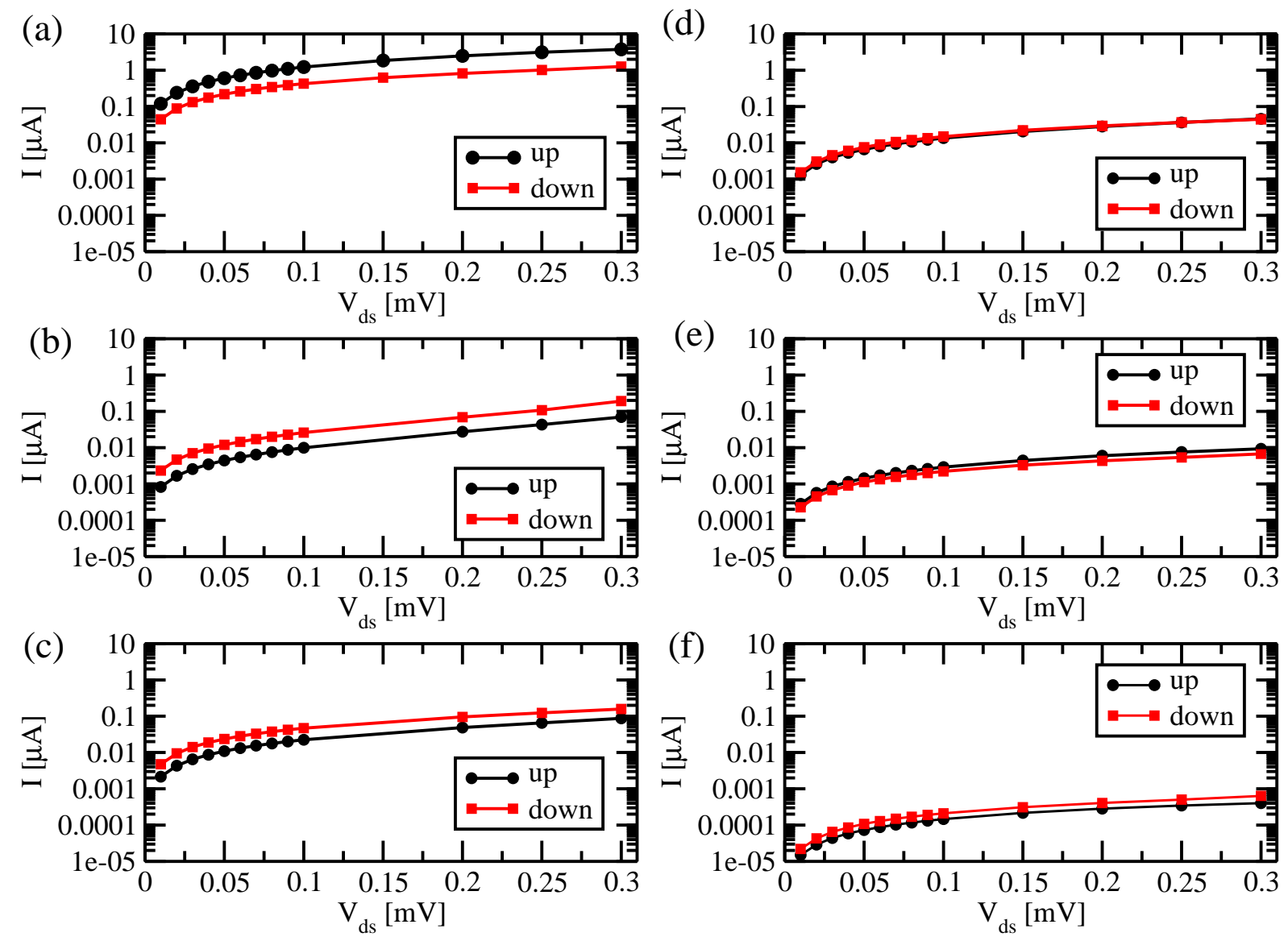

Figura 8.15: Corrente em função da tensão de bias para: (a) 1, (b) 2, (c) 3, (d) 4, (e) 5 e (f) 6 células unitárias de BN. O eixo da corrente está em escala log para uma melhor visualização.

zação da corrente. Na figura 8.16, apresentamos os resultados para a polarização da corrente em função da voltagem de bias para os sistemas com alinhamento paralelo dos eletrodos. Um ponto importante é que as maiores polarizações de corrente são observadas para 1 e 2 camadas de $B N$, onde para 1 camada a corrente up maior e para 2 camadas a corrente down é predominante. Como podemos observar através dos resultados, a polarização da corrente não é tão grande, da ordem de $50 \%$ para o maior valor. Mesmo assim, este dispositivo ainda pode ser um bom material para atuação em filtros de spin. Um outro ponto importante é que a polarização cai quando aumentamos o número de camadas de BN.

Uma outra aplicação importante de junções túnel magnéticas é em dispositivos que utilizam o efeito da magnetoresistência. Na figura 8.17, apresentamos os resultados para a magnetoresistência túnel, definida como:

$$
T M R[\%]=\frac{I_{P}-I_{A P}}{I_{A P}} \times 100
$$




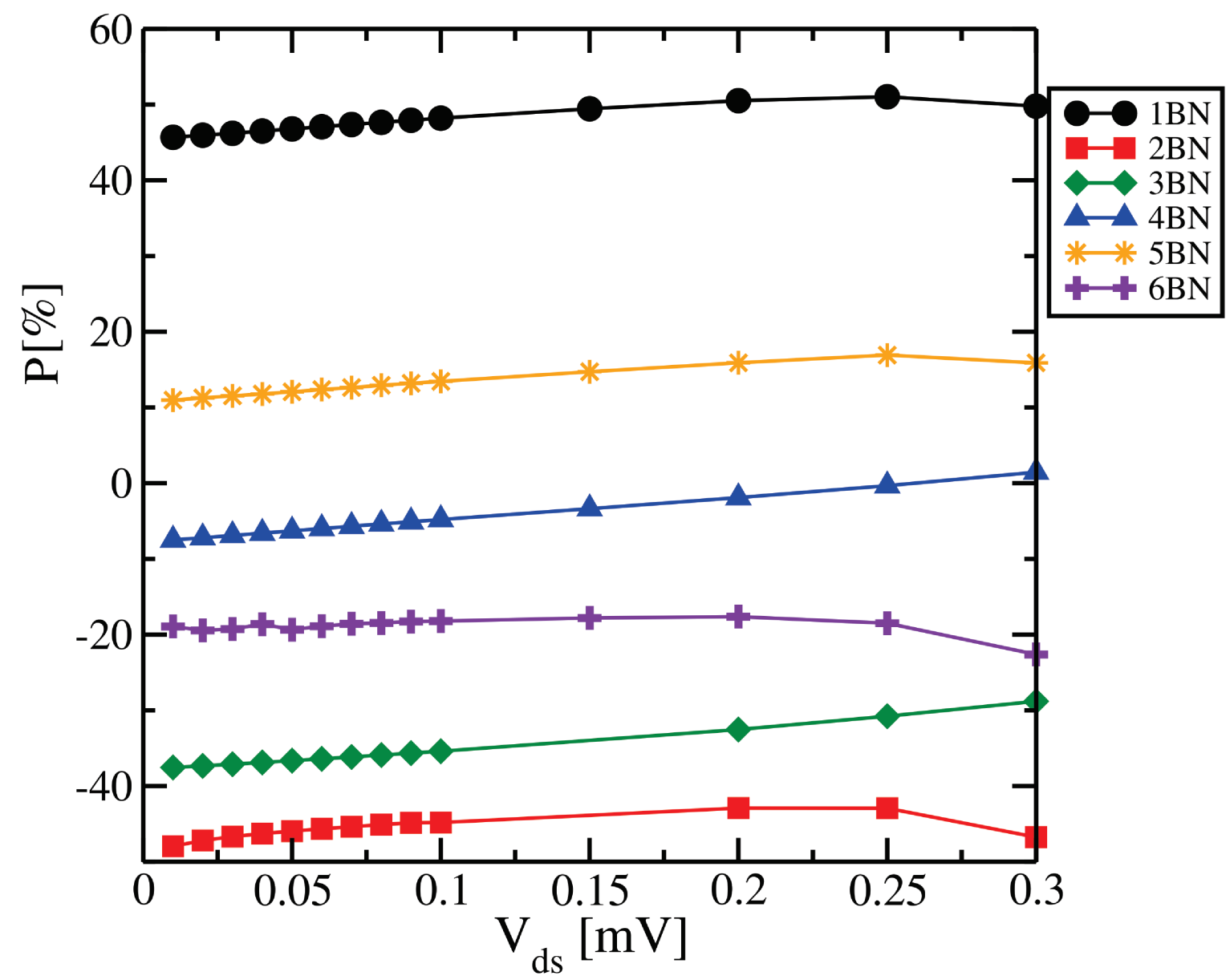

Figura 8.16: Polarização da corrente em função da voltagem de bias, $V_{d s}$, para $N$ uc de $\operatorname{BN}(N=$ $1,2, \cdots, 6)$. A polarização é definida como: $P[\%]=\frac{I_{\uparrow}-I_{\downarrow}}{I_{\uparrow}+I_{\downarrow}}$.

onde $I_{P}$ corresponde à corrente com alinhamento paralelo dos eletrodos e $I_{A P}$ alinhamento antiparalelo.

Em contraste com a polarização da corrente, que apresenta seu maior valor no caso de uma única célula unitária de $\mathrm{BN}$, o maior valor da TMR ocorre para 4 células unitárias, chegando a valores da ordem de $\approx 5 \times 10^{5 \%}$. Isto nos leva a concluir que os sistemas estudados podem não ser muito eficientes como filtros de spin, porém apresentam efeitos de magnetoresistência gigante. Dessa forma, seu uso pode ser promisor em tais dispositivos.

\subsection{Conclusões}

Em resumo, realizamos cálculos de primeiros princípios para a estrutura eletrônica e transporte eletrônico para as nanofitas de grafeno $(3,0)$ zigzag pristinas com eletrodos alinhados 


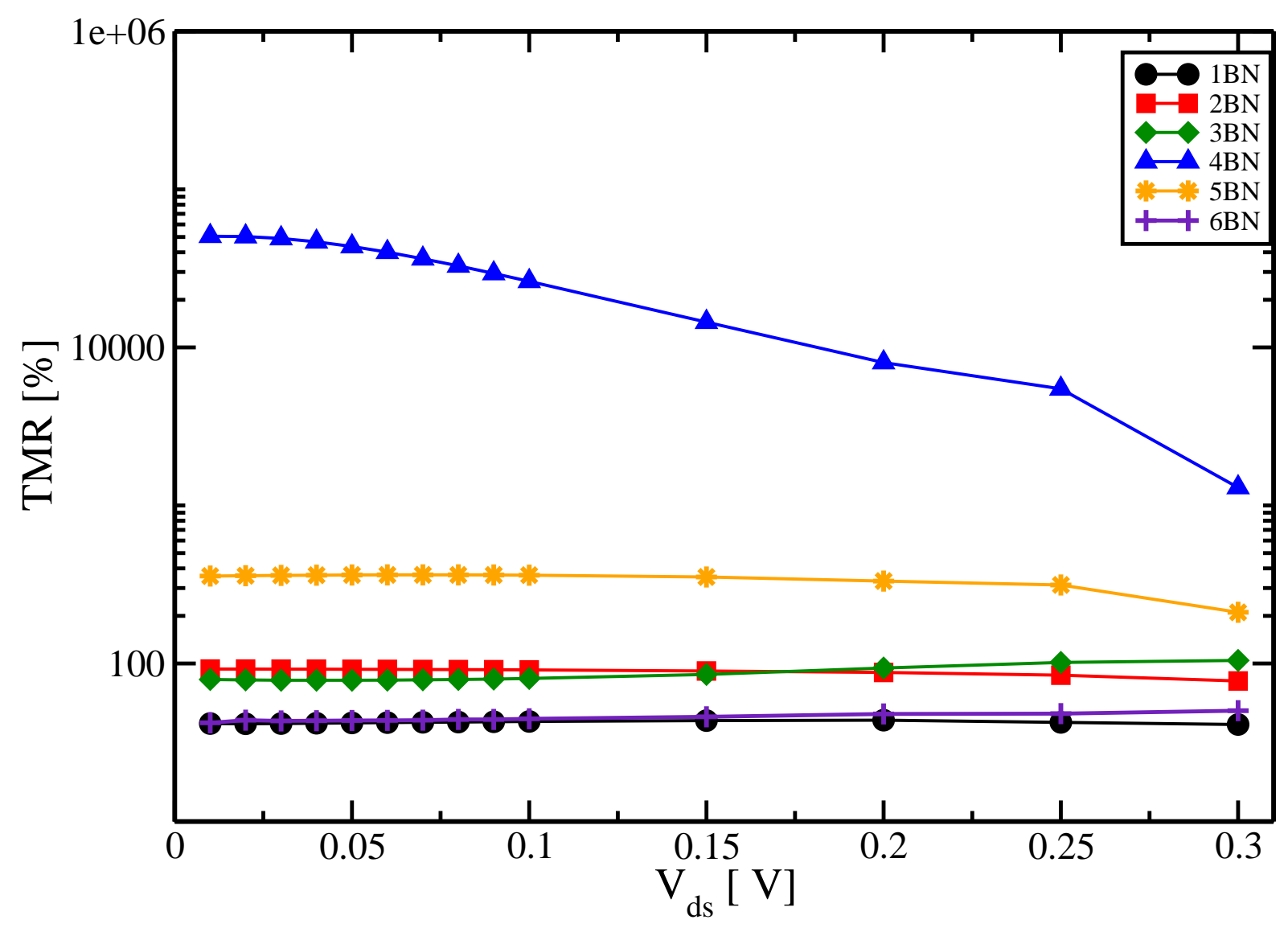

Figura 8.17: Magnetoresistência de tunelamento (TMR) em função da voltagem fonte dreno, $V_{d s}$, para $N$ uc de $\mathrm{BN}(N=1,2, \cdots, 6)$.

ferromagneticamente e antiferromagneticamente, tal como para as GNR's dopadas com boro e nitrogênio. Para o sistema pristino com eletrodos na configuração FM, devido a um valor constante da transmitância em torno do nível de Fermi, a corrente para tensões baixas de bias apresenta um comportamento linear e, além disso, ambas as correntes para os canais up e down são degeneradas. Entretanto, para bias entre $\pm 0.5 \mathrm{~V}$ e $\pm 2.0 \mathrm{~V}$, as correntes são praticamente constantes, sendo a corrente up sempre maior que a down. Analisando a configuração AFM dos eletrodos, verificamos a presença de um efeito de filtro de spin, com a corrente sendo transportada por somente um canal de spin, tanto para bias negativo quanto positivo. A dopagem do sistema com boro e nitrogênio, leva o sistema a apresentar correntes não degeneradas em todo o range de voltagens utilizada. Para a dopagem com boro, com bias positivo até $1.5 \mathrm{~V}$, a corrente down é maior que a up, enquanto que para o nitrogênio o comportamento é o oposto. Isso ocorre devido ao fato que o boro introduz um buraco no sistema e o nitrogênio um elétron. Além disso, para ambas as dopagens, nós observamos uma inversão na polarização da corrente 
acima e abaixo de alguma voltagem de bias, específica para cada tipo de dopagem.

Finalmente, através do estudo de uma junção túnel magnética, observamos que o sistema composto de nanofitas de grafeno e nanofitas de h-BN pode ser utilizada tanto como filtros de spin como elementos para dispositivos de magnetoresistência gigante, onde para este último a sua eficiência é muito mais pronunciada para um sistema com 4 camadas de $\mathrm{BN}$, apresentando valores de magnetoresistência de $10^{5 \%}$. 


\section{Capítulo 9}

\section{Conclusões}

Nesta tese de doutorado investigamos a partir de cálculos de primeiros princípios, dispositivos e componentes de dispositivos baseados em grafeno. Abordamos tanto os campos da nanoeletrônica como o da spintrônica.

No campo da nanoeletrônica investigamos:

(i) Nanotransistor de bicamada de grafeno: Mostramos que sobre a ação de um campo elétrico externo, mesmo para comprimentos de gate de $100 \AA$, à temperatura ambiente e a $4.5 \mathrm{~K}$, uma corrente não nula sempre é exibida. Os resultados podem ser explicados pela presença de um regime de tunelamento devido a estados remanescentes na região do gap. Obtemos também razões on/off para a corrente de 100 à $300 \mathrm{~K}$ e 1250 à $4.5 \mathrm{~K}$, que são valores próximos aos observados experimentalmente [72], que estudaram este mesmo tipo de sistema.

(ii) Multicamadas de grafeno: Para as propriedades eletrônicas das multicamadas de grafeno, mostramos que as estruturas de bandas das FLG são fortemente dependentes do empilhamento das camadas. Mostramos que na presença de um campo elétrico externo, as FLG com empilhamento de Bernal nunca apresentam a abertura de um gap de energia devido a uma sobreposição das bandas, enquanto que as FLG com o empilhamento romboédrico é possível a obtenção de um gap de energia controlável, porém limitado pelo número de camadas do sistema, onde quanto mais camadas menor é o gap induzido. Do mesmo modo que a estrutura eletrônica do sistema, as propriedades de transporte são fortemente dependentes do tipo de empilhamento nas camadas. No sistema Bernal, o transporte ocorre por todas as camadas, enquanto que no caso romboédrico o transporte é 
predominantemente de superfície. Um ponto importante é que é possível diferenciar um empilhamento do outro através da corrente ou resistência do sistema. Além disso, mostramos também que é possível utilizar a temperatura para diferenciar um empilhamento do outro.

(iii) Componentes mais realistas de um nanodispositivo: dentro das componentes de um nanotransistor mais realista, onde existem os substratos e os contatos metálicos, estudamos as propriedades eletrônicas e estruturais de bicamadas de grafeno depositadas sobre substratos de nitreto de boro hexagonal e também heteroestruturas compostas de bicamadas de grafeno, nitreto de boro hexagonal e uma superfície de cobre. As principais conclusões sobre esses sistemas são:

- (a) a partir de cálculos de primeiros princípios de estrutura eletrônica baseados em DFT, mostramos que é possível criar um gap de energia controlável na bicamada de grafeno sobre um substrato de nitreto de boro hexagonal, que este gap fica ligeiramente maior quanto maior é o número de camadas de h-BN. Mostramos também que na presença de um campo elétrico, níveis do substrato de h-BN são inseridos no nível de Fermi do sistema, fazendo com que este fique metálico. Além disso, o range de voltagens que podem ser aplicadas no dispositivo depende fortemente do número de camadas de hBN e da direção do campo elétrico. Finalmente, um comportamento fantástico, é que a voltagem de quebra do dielétrico e a possibilidade da criação do gap na bicamada é independente do tipo de empilhamento do substrato de h-BN.

- (b) Dentro das propriedades eletrônicas e estruturais de heteroestruturas compostas de bicamdas de grafeno, nitreto de boro hexagonal e cobre, primeiramente observamos que em todos os sistemas considerados em nossas investigações, independentemente do número de camadas de h-BN no sistema, as bicamadas de grafeno ficam sempre dopadas tipo $n$, nos sistemas na ausência de campo elétrico. Mostramos que é possível controlar o tipo de dopagem das bicamadas, indo de tipo $n$ até tipo $p$, passando por um estado neutro, para todas as configurações que estudamos, exceto nos sistemas $\mathrm{Cu}(111) / \mathrm{h}-\mathrm{BN} / \mathrm{BG} / \mathrm{h}-\mathrm{BN}$ com mais de 1 camada de h-BN. Segundo, observamos que é possível controlar o valor do gap de energia nas bicamadas de grafeno através de um campo elétrico externo, onde sintonizando a intensidade e a direção do campo conseguimos tanto zerar o gap induzido 
pelo campo elétrico gerado na região de depleção criada na interface entre o cobre e o restante do sistema, como é possível também aumentar o valor deste gap de energia. Finalmente, dentre todos os sistemas estudados, no sentido de aplicação direta em um dispositivo, concluímos que a melhor configuração é a $\mathrm{Cu}(111) / \mathrm{h}-\mathrm{BN} / \mathrm{BG}$, representada na figura 7.2(c). Neste sistema independentemente do número de camadas de h-BN, possuímos todo o controle sobre a bicamada, ou seja, valor do gap de energia e tipo de dopagem, sem a adição de estados vindos do h-BN, criando um canal de condução através do dielétrico.

No campo da spintrônica:

(i) Realizamos cálculos de primeiros princípios para a estrutura eletrônica e transporte eletrônico das nanofitas de grafeno $(3,0)$ zigzag pristinas com eletrodos alinhados ferromagneticamente e antiferromagneticamente, tal como para as GNR dopadas com boro e nitrogênio. Para o sistema pristino com eletrodos na configuração FM, devido a um valor constante da transmitância ao redor do nível de Fermi, a corrente para tensões de bias baixas apresenta um comportamento linear e além disso, ambas as correntes para os canais up e down são degeneradas. Entretanto, para bias entre $\pm 0.5 \mathrm{~V}$ e $\pm 2.0 \mathrm{~V}$, as correntes são praticamente constantes, sendo a corrente up sempre maior que a down. Analisando a configuração AFM dos eletrodos, nós verificamos a presença de um efeito de filtro de spin, com a corrente sendo transportada por somente um canal de spin, tanto para bias negativos quanto positivos. A dopagem do sistema com boro e nitrogênio, leva o sistema a apresentar correntes não degeneradas em todo o range de voltagens que utilizamos. Para a dopagem com boro, com bias positivo baixo, a corrente down é maior que a up, enquanto que para o nitrogênio o comportamento é o oposto. Isso ocorre devido ao fato que o boro introduz um buraco no sistema e o nitrogênio um elétron. Além disso, para ambas as dopagens, nós observamos uma inversão na polarização da corrente acima e abaixo de alguma voltagem de bias, específica para cada tipo de dopagem.

(ii) Através do estudo de uma junção túnel magnética, observamos que o sistema composto de nanofitas de grafeno e nanofitas de h-BN pode ser utilizada tanto como filtros de spin como elementos para dispositivos de magnetoresistência gigante, onde para este último a sua eficiência é muito mais pronunciada. 


\section{Apêndice A}

\section{Multigrid}

Os métodos de multigrid foram originalmente desenvolvidos para a obtenção da solução de problemas diferenciais de uma maneira simples e eficiente. As suas principais aplicações são: Equações diferenciais parciais elípticas (EDP); problemas puramente algébricos sem a necessidade de um grid com um significado físico; reconstrução de imagens em tomografias e ressonâncias magnéticas; otimização de processos; cromodinâmica quântica; mecânica estatística e problemas de Ising; FFT's generalizadas e problemas de quadraturas; equações integrais e muitos outros problemas relacionados com as diversas áreas da engenharia, física, etc. $[65,143,144]$.

Neste apêndice, introduziremos os conceitos básicos do método de multigrid que são utilizados para soluções de equações diferenciais parciais elípticas. Esta técnica foi implementada no código SIESTA, para a solução da equação de Poisson no espaço real.

\section{A.1 Discretização do Problema}

Nesta seção, utilizaremos dois problemas modelo para definirmos como é feita a discretização do problema diferencial.

\section{A.1.1 Problema Modelo 1 - Caso Unidimensional}

O métodos de multigrid foram originalmente aplicados a problemas de valores de contorno simples que surgiram em muitas aplicações físicas. Esses problemas fornecem uma introdução natural aos métodos de multigrid e servem como um ótimo laboratório didático para o entendi- 
mento da técnica ${ }^{1}$.

Na solução de um problema numérico um elemento básico e muito importante, é como a discretização do problema é feita. Aqui iremos expor como é realizada a discretização do problema em um grid cartesiano unidimensional. Para uma descrição mais geral, vamos considerar um problema de valor de contorno que é dado por uma equação diferencial de segunda ordem²:

$$
\begin{array}{r}
-u^{\prime \prime}(x)+\sigma u(x)=f(x), \quad 0<x<1, \quad \sigma \geq 0, \\
u(0)=u(1)=0 . \quad \text { Contornos do problema }
\end{array}
$$

Esta equação pode ser facilmente resolvida de forma analítica, mas iremos considerar aqui um método numérico. Existem vários tipos de abordagens numéricas para resolver problemas desse tipo, onde o mais simples é o de diferenças finitas. Para isso, o domínio do problema $\{x: 0 \leq x \leq 1\}$, é particionado em $n$ subintervalos introduzindo-se os pontos do grid $x_{j}=$ $j h$, onde $h=1 / n$ é a constante de comprimento dos subintervalos. Isso estabelece o grid apresentado na figura A.1, que denotaremos por grid $\Omega^{h}$.

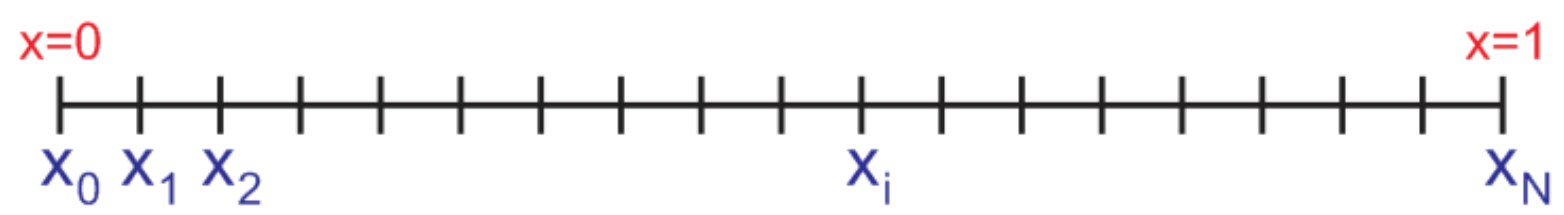

Figura A.1: Grid unidimensional.

Em cada um dos $n-1$ pontos do interioriores do grid, a equação diferencial original, (A.1), pode ser substituída por uma aproximação de diferenças finitas de segunda ordem. Para obtermos essa aproximação, utilizamos uma expansão em séries de Taylor para os pontos $x_{i+1}$ e $x_{i-1}$ :

$$
\begin{aligned}
& u\left(x_{i-1}\right)=u\left(x_{i}\right)-h u^{\prime}\left(x_{i}\right)+\frac{h^{2}}{2 !} u^{\prime \prime}\left(x_{i}\right)-\frac{h^{3}}{3 !} u^{\prime \prime \prime}\left(x_{i}\right)+O\left(h^{4}\right) \\
& u\left(x_{i+1}\right)=u\left(x_{i}\right)+h u^{\prime}\left(x_{i}\right)+\frac{h^{2}}{2 !} u^{\prime \prime}\left(x_{i}\right)+\frac{h^{3}}{3 !} u^{\prime \prime \prime}\left(x_{i}\right)+O\left(h^{4}\right)
\end{aligned}
$$

\footnotetext{
${ }^{1}$ Um exemplo de problema físico é a descrição da distribuição de temperatura no estado estacionário em uma haste uniforme.

${ }^{2}$ Iremos utilizar em todo o texto a notação $u^{\prime \prime}(x)=\frac{d^{2} u(x)}{d x^{2}}$ para as formas derivadas totais e $u_{x x}=\frac{\partial^{2} u}{\partial u^{2}}$ para as formas que envolvem derivadas parciais
} 
Somando as equações (A.3) e (A.4) e isolando $u^{\prime \prime}\left(x_{i}\right)$, obtemos:

$$
u^{\prime \prime}\left(x_{i}\right)=\frac{u\left(x_{i+1}\right)-2 u\left(x_{i}\right)+u\left(x_{i-1}\right)}{h^{2}}+O\left(h^{2}\right),
$$

que é a aproximação de diferença finita de segunda ordem para a segunda derivada.

Antes de substituir essa aproximação na equação original, introduzimos $v_{i}$ como sendo uma aproximação para a solução exata $u\left(x_{i}\right)$. Além disso, podemos impor as condições de contorno do problema, onde nesse caso, utilizaremos uma condição de contorno de Dirichlet, especificada nos extremos do grid:

$$
v_{0}=v_{N}=0
$$

De posse dessas condições de contorno, a equação do problema pode ser escrita no esquema de diferenças finitas como:

$$
\begin{array}{r}
\frac{-v_{i-1}+2 v_{i}-v_{i+1}}{h^{2}}+\sigma v_{i}=f_{i} \quad i=1,2, \cdots, N-1 \\
v_{0}=v_{N}=0
\end{array}
$$

Este problema, equação (A.7), pode ainda ser escrito em uma forma matricial. Para isso tomamos $\mathbf{v}=\left(v_{1}, v_{2}, \cdots, v_{N-1}\right)^{T} \mathbf{e} \mathbf{f}=\left(f_{1}, f_{2}, \cdots, f_{N-1}\right)^{T}$. Dessa forma temos uma equação matricial $\mathbf{A v}=\mathbf{f}$, onde $\mathbf{A}$ é uma matriz $(N-1) \times(N-1)$, simétrica e positiva definida, onde

$$
\mathrm{Av}=\mathbf{f}
$$

$$
\begin{aligned}
& \frac{1}{h^{2}}\left(\begin{array}{cccccc}
2+\sigma h^{2} & -1 & & & & \\
& 2+\sigma h^{2} & -1 & & & \\
& -1 & 2+\sigma h^{2} & -1 & & \\
& & & & & \\
& & & -1 & 2+\sigma h^{2} & -1 \\
& & & -1 & 2+\sigma h^{2}
\end{array}\right)\left(\begin{array}{c}
v_{1} \\
v_{2} \\
v_{3} \\
\\
v_{N-2} \\
v_{N-1}
\end{array}\right)= \\
& \left(\begin{array}{c}
f_{1} \\
f_{2} \\
f_{3} \\
f_{N-2} \\
f_{N-1}
\end{array}\right)
\end{aligned}
$$

Dada a discretização do problema unidimensional, podemos extender este problema ao caso bidimensional. 


\section{A.1.2 Problema Modelo 2 - Caso Bidimensional}

Nesta seção estudaremos como é feita a discretização do problema bidimensional. De certo modo, fazendo isso temos todos os ingredientes para tratar os problemas tridimensionais. Assim, da mesma maneira que descrevemos o problema na seção A.1.1, podemos definir o problema bidimensional como:

$$
\begin{array}{r}
-u_{x x}-u_{y y}+\sigma u=f(x, y), \quad 0<x<1, \quad 0<y<1 \\
u=0 \rightarrow x=0, x=1, y=0, y=1 ; \quad \sigma>0
\end{array}
$$

O grid bidimensional é ilustrado na figura A.2. sendo definido pelos parâmetros:

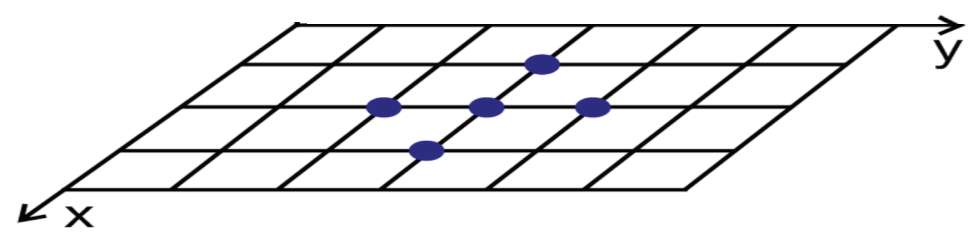

Figura A.2: Grid bidimensional.

$$
\begin{array}{r}
h_{x}=\frac{1}{M}, \quad h_{y}=\frac{1}{N} \quad\left(x_{i}, y_{j}\right)=\left(i h_{x}, j h_{y}\right) \\
0 \leq i \leq M \quad 0 \leq j \leq N
\end{array}
$$

A discretização da equação é realizada, utilizando-se o mesmo esquema de diferenças finitas do caso unidimensional. Seja $v_{i j} \approx u\left(x_{i}, y_{j}\right)$ e $f_{i j} \approx f\left(x_{i}, y_{j}\right)$ e a partir de uma expansão em séries de Taylor para $u_{x x}$ e $u_{y y}$ a equação na forma discreta fica:

$$
\begin{array}{r}
\frac{-v_{i-1, j}+2 v_{i j}-v_{i+1, j}}{h_{x}^{2}}+\frac{-v_{i, j-1}+2 v_{i j}-v_{i, j+1}}{h_{y}^{2}}+\sigma v_{i j}=f_{i j} \\
v_{i j}=0 \rightarrow i=0, i=M, j=0, j=N
\end{array}
$$

Novamente, escrevendo na forma matricial, ordenamos as incógnitas $v_{i j}$ na forma de um vetor como,

$$
\mathbf{v}=\left(v_{1,1}, v_{1,2}, \cdots, v_{1, N-1}, v_{2,1}, v_{2,2}, \cdots, v_{2, N-1, \cdots, v_{N-1, N-1}}\right)^{T}
$$


e da mesma maneira $f_{i j}$ o sistema resultante fica em uma forma bloco-tridiagonal:

$$
\left(\begin{array}{ccccc}
A_{1} & -I_{y} & & & \\
-I_{y} & A_{2} & -I_{y} & & \\
& -I_{y} & A_{3} & -I_{y} & \\
& & \ddots & \ddots & \ddots \\
& & -I_{y} & A_{N-2} & -I_{y} \\
& & & -I_{y} & A_{N-1}
\end{array}\right)\left(\begin{array}{c}
v_{1} \\
v_{2} \\
v_{3} \\
\vdots \\
v_{N-2} \\
v_{N-1}
\end{array}\right)=\left(\begin{array}{c}
f_{1} \\
f_{2} \\
f_{3} \\
\vdots \\
f_{N-2} \\
f_{N-1}
\end{array}\right)
$$

onde $I_{y}$ é uma matriz diagonal com $\frac{1}{h_{y}^{2}}$ na diagonal, e:

$$
A_{i}=\left(\begin{array}{ccc}
\frac{1}{h_{x}^{2}}+\frac{1}{h_{y}^{2}}+\sigma & -\frac{1}{h_{x}^{2}} & \\
-\frac{1}{h_{x}^{2}} & \frac{1}{h_{x}^{2}}+\frac{1}{h_{y}^{2}}+\sigma & -\frac{1}{h_{x}^{2}} \\
\ddots & \ddots & \ddots
\end{array}\right)
$$

Novamente, realizamos somente a descrição do problema, exibindo como fica a forma discreta dos operadores. Os métodos para a solução do problema serão descritos na seção A.2.

\section{A.2 Métodos Iterativos para Sistemas Lineares}

Antes de introduzirmos de fato os métodos iterativos ${ }^{3}$, apresentaremos duas grandezas que são importantes para o entendimento destes métodos e da técnica de multigrid.

\section{A.2.1 Erro e Resíduo}

Considere $\mathbf{A u}=\mathbf{f}$, com dimensão $(N \times N)$, e $\mathbf{v}$ como uma aproximação para a solução exata u. A primeira medida importante é o erro da aproximação, dado por:

$$
\mathbf{e}=\mathbf{u}-\mathbf{v}
$$

que possui norma $\|e\|_{2}$ dada por:

$$
\|e\|_{2}=\left[\sum_{i=1}^{n} e_{i}^{2}\right]^{1 / 2}
$$

A segunda medida importante é chamada de resíduo, dado por:

$$
\mathbf{r}=\mathbf{f}-\mathbf{A v} .
$$

\footnotetext{
${ }^{3}$ Comumente chamados de métodos de relaxação.
} 
Essas duas medidas nos levam ao esquema que chamamos de correção residual, ou seja,

$$
\mathbf{e}=\mathbf{u}-\mathbf{v}
$$

$\mathrm{e}$

$$
\mathbf{r}=\mathbf{f}-\mathbf{A v}
$$

A equação $\mathbf{A u}=\mathbf{f}$ pode ser escrita como

$$
\begin{array}{r}
\mathbf{e}=\mathbf{u}-\mathbf{v} \rightarrow \mathbf{u}=\mathbf{v}+\mathbf{e} \\
\mathbf{A} \mathbf{u}=\mathbf{f} \rightarrow \mathbf{A}(\mathbf{v}+\mathbf{e})=\mathbf{f} \\
\mathbf{A v}+\mathbf{A e}=f \rightarrow \mathbf{f}-\mathbf{r}+\mathbf{A e}=\mathbf{f} \\
\mathbf{A e}=\mathbf{r} \quad \text { Equação Residual }
\end{array}
$$

Dessa maneira, se resolvermos $\mathbf{A e}=\mathbf{r}$, obtemos o erro da aproximação, e de posse do erro, corrigimos a solução:

$$
\mathbf{u}=\mathbf{v}+\mathbf{e} \quad \text { Correção Residual }
$$

Dado essas medidas ${ }^{4}$, podemos entrar de fato nos métodos iterativos para a solução do problema.

Dentre os vários métodos que existem para a solução de problemas de valores de contorno, existem duas grandes classes: (i) Métodos diretos (Eliminação gaussiana, fatoriazação, etc.); (ii) Métodos iterativos (Jacobi, Gauss-Seidel, Gradiente Conjugado, etc.) [145]. Neste trabalho iremos realizar a descrição dos métodos iterativos de Jacobi e Gauss-Seidel, pois eles apresentam uma grande eficiência nas técnicas de multigrid e a sua implementação é relativamente simples.

\section{A.2.2 Método de Jacobi}

Para iniciarmos a descrição do método de Jacobi, vamos considerar o problema $1 D$, discreto, dado pela equação abaixo:

$$
\begin{array}{r}
\frac{-v_{i-1}+2 v_{i}-v_{i+1}}{h^{2}}+\sigma v_{i}=f_{i} \quad i=1,2, \cdots, N-1 \\
v_{1}=v_{N}=0
\end{array}
$$

\footnotetext{
${ }^{4}$ Erro, resíduo, equação residual e correção residual.
} 
Por simplicidade, vamos tomar $\sigma=0^{5}$. Assim, o problema exato fica:

$$
\begin{array}{r}
\frac{-u_{i-1}+2 u_{i}-u_{i+1}}{h^{2}}=f_{i} \\
-u_{i-1}+2 u_{i}-u_{i+1}=h^{2} f_{i}
\end{array}
$$

O método de Jacobi ${ }^{6}$ nos diz: "Resolva a $i$-ésima equação para $v_{i}$, mantendo todas as outras variáveis fixas, ou seja:

$$
\begin{array}{r}
-v_{i-1}+2 v_{i}-v_{i+1}=h^{2} f_{i} \\
v_{i}=\frac{1}{2}\left(h^{2} f_{i}+v_{i-1}+v_{i+1}\right) \\
v_{i}^{1}=\frac{1}{2}\left(h^{2} f_{i}+v_{i-1}^{0}+v_{i+1}^{0}\right) \quad 1 \leq i \leq N-1
\end{array}
$$

onde, $v^{1}$ representa a nova aproximação e $v^{0}$ a aproximação antiga.

O operador A pode ser escrito em uma forma matricial como:

$$
\mathbf{A}=(\mathbf{D}-\mathbf{L}-\mathbf{U})
$$

onde $\mathbf{D}$ é a diagonal da matriz e $\mathbf{L}$ e $\mathbf{U}$ são as partes inferior e superior, respectivamente. Assim, $\mathbf{A u}=\mathbf{f}$, pode ser escrito como:

$$
\begin{array}{r}
(\mathbf{D}-\mathbf{L}-\mathbf{U}) \mathbf{u}=\mathbf{f} \\
\mathbf{D} \mathbf{u}-(\mathbf{L}+\mathbf{U}) \mathbf{u}=\mathbf{f} \\
\mathbf{D} \mathbf{u}=\mathbf{f}+(\mathbf{L}+\mathbf{U}) \mathbf{u} \\
\mathbf{u}=\mathbf{D}^{-1}(\mathbf{L}+\mathbf{U}) \mathbf{u}+\mathbf{D}^{-1} \mathbf{f}
\end{array}
$$

e se compararmos essa equação com a equação (A.23), observamos que uma é equivalente à outra. Assim,

$$
\mathbf{v}^{1}=\mathbf{D}^{-1}(\mathbf{L}+\mathbf{U}) \mathbf{v}^{0}+\mathbf{D}^{-1} \mathbf{f}
$$

Definindo $\mathbf{R}_{J}=\mathbf{D}^{-1}(\mathbf{L}+\mathbf{U})$, onde $\mathbf{R}_{J}$ é o operador de Jacobi, a iteração de Jacobi na forma matricial pode ser escrita como:

$$
\mathbf{v}^{1}=\mathbf{R}_{J} \mathbf{v}^{0}+\mathbf{D}^{-1} \mathbf{f}
$$

\footnotetext{
${ }^{5}$ Esta aproximação, $\sigma=0$, deixa o problema muito parecido com a equação de Poisson.

${ }^{6}$ Também conhecido como método dos deslocamentos simultâneos.
} 
A partir dessa derivação, podemos como fica o erro nesta iteração. Da solução exata do problema:

$$
\begin{array}{r}
\mathbf{u}=\mathbf{D}^{-1}(\mathbf{L}+\mathbf{U}) \mathbf{u}+\mathbf{D}^{-1} \mathbf{f} \\
\mathbf{u}=\mathbf{R}_{J} \mathbf{u}+\mathbf{D}^{-1} \mathbf{f}
\end{array}
$$

e da aproximação:

$$
\mathbf{v}^{1}=\mathbf{R}_{J} \mathbf{v}^{0}+\mathbf{D}^{-1} \mathbf{f}
$$

O erro é dado por:

$$
\begin{array}{r}
\mathbf{e}=\mathbf{u}-\mathbf{v} \\
\mathbf{u}-\mathbf{v}^{1}=\mathbf{R}_{J} \mathbf{u}+\mathbf{D}^{-1} \mathbf{f}-\mathbf{R}_{J} \mathbf{v}^{0}-\mathbf{D}^{-1} \mathbf{f} \\
\mathbf{u}-\mathbf{v}^{1}=\mathbf{R}_{J} \mathbf{u}-\mathbf{R}_{J} \mathbf{v}^{0} \\
\mathbf{e}^{1}=\mathbf{R}_{J} \mathbf{e}^{0}
\end{array}
$$

Existe ainda uma modificação simples, porém muito importante, que pode ser feita na iteração de Jacobi. Como fizemos anteriormente, podemos calcular a iteração de Jacobi como:

$$
v_{i}^{*}=\frac{1}{2}\left(v_{i-1}^{0}+v_{i+1}^{0}+h^{2} f_{i}\right),
$$

onde $v_{i}^{*}$ não é exatamente a nova aproximação, mas somente um valor intermediário entre $v^{0}$ e $v^{1}$. Assim, a nova iteração pode ser escrita como uma combinação da iteração intermediária (*) com a iteração antiga $(0)$, usando um fator de peso apropriado. A iteração final pode ser escrita por:

$$
\begin{aligned}
v_{i}^{1}=v_{i}^{0}-\omega v_{i}^{0}+\omega v_{i}^{*} & =v_{i}^{0}-\omega v_{i}^{0}+\omega \frac{1}{2}\left(v_{i-1}^{0}+v_{i+1}^{0}+h^{2} f_{i}\right) \\
v_{i}^{1} & =(1-\omega) v_{i}^{0}+\frac{\omega}{2}\left(v_{i-1}^{0}+v_{i+1}^{0}+h^{2} f_{i}\right)
\end{aligned}
$$

que é conhecida como iteração de Jacobi Ponderada ${ }^{7}$.

\footnotetext{
${ }^{7}$ Mais conhecida por seu termo em inglês: Weighted Jacobi
} 
Na forma matricial esta iteração pode ser escrita como:

$$
\begin{array}{r}
v_{i}^{1}=(1-\omega) v_{i}^{0}+\frac{\omega}{2}\left(v_{i-1}^{0}+v_{i+1}^{0}\right) \frac{\omega}{2} h^{2} f_{i} \\
\mathbf{v}^{1}=(1-\omega) \mathbf{v}^{0}+\omega \mathbf{D}^{-1}(\mathbf{L}+\mathbf{U}) \mathbf{v}^{0}+\omega \mathbf{D}^{-1} \mathbf{f} \\
\mathbf{v}^{1}=\left[(1-\omega) \mathbf{I}+\omega \mathbf{R}_{J}\right] \mathbf{v}^{0}+\omega \mathbf{D}^{-1} \mathbf{f} \\
\mathbf{v}^{1}=\mathbf{R}_{\omega} \mathbf{v}^{0}+\omega \mathbf{D}^{-1} \mathbf{f}
\end{array}
$$

onde a matriz de iteração $\mathbf{R}_{\omega}$ é dada por

$$
\mathbf{R}_{\omega}(1-\omega) \mathbf{I}+\omega \mathbf{R}_{J}
$$

Sabendo que o resíduo associado com $\mathbf{v}^{0}$ é dado por:

$$
\mathbf{r}^{0}=\mathbf{f}-\mathbf{A} \mathbf{v}^{0}
$$

e usando $\mathbf{A}=\mathbf{D}-\mathbf{L}-\mathbf{U}$, temos

$$
\begin{array}{r}
\mathbf{r}^{0}=\mathbf{f}-(\mathbf{D}-\mathbf{L}-\mathbf{U}) \mathbf{v}^{0} \\
\mathbf{r}^{0}=\mathbf{f}-\mathbf{D} \mathbf{v}^{0}+(\mathbf{L}+\mathbf{U}) \mathbf{v}^{0} \\
\mathbf{D} \mathbf{v}^{0}=\mathbf{f}+(\mathbf{L}+\mathbf{U}) \mathbf{v}^{0}-\mathbf{r}^{0} \\
\mathbf{v}^{0}=\mathbf{D}^{-1} \mathbf{f}+\mathbf{D}^{-1}(\mathbf{L}+\mathbf{U}) \mathbf{v}^{0}-\mathbf{D}^{-1} \mathbf{r}^{0} \\
\mathbf{v}^{0}=\mathbf{D}^{-1} \mathbf{f}-\mathbf{D}^{-1} \mathbf{r}^{0}+\mathbf{R}_{J} \mathbf{v}^{0}
\end{array}
$$

Assim da iteração de Jacobi ponderada dada pela equação (A.32), a nova aproximação $\mathbf{v}^{1}$ pode ser escrita como:

$$
\begin{array}{r}
\mathbf{v}^{1}=\left[(1-\omega) \mathbf{I}+\omega \mathbf{R}_{J}\right] \mathbf{v}^{0}+\omega \mathbf{D}^{-1} \mathbf{f} \\
\mathbf{v}^{1}=\mathbf{v}^{0}-\omega \mathbf{v}^{0}+\omega \mathbf{R}_{J} \mathbf{v}^{0}+\omega \mathbf{D}^{-1} \mathbf{f} \\
\mathbf{v}^{1}=\mathbf{v}^{0}+\omega \mathbf{D}^{-1} \mathbf{r}^{0}
\end{array}
$$

Esta equação nos diz: A nova aproximação, é obtida a partir da aproximação antiga, mais uma quantidade apropriada do seu resíduo. 
O erro desta aproximação é dado por:

$$
\mathbf{e}^{1}=\mathbf{R}_{\omega} \mathbf{e}^{0}
$$

\section{A.2.3 Iteração de Gauss-Seidel}

O método de Gauss-Seidel, é muito semelhante ao de Jacobi, porém incorpora uma modificação simples que faz toda a diferença: As componentes da nova aproximação são utilizadas assim que são calculadas. Isso significa que as componentes da nova aproximação $\mathbf{v}$ são sobrescritas assim que são calculadas. Isso reduz muito a quantidade de armazenamento de informação, pois precisamos ter somente o vetor $\mathbf{v}$ na memória.

A iteração de Gauss-Seidel é equivalente a definirmos sucessivamente cada componente de resíduo para zero, e resolver a correspondente componente da solução. Se aplicarmos o método de Gauss-Seidel ao problema $1 D$ que tratamos anteriormente, temos o seguinte: Resolve-se a $i$-ésima equação para $v_{i}$ e atualiza o seu valor imediatamente em $\mathbf{v}^{8}$. Na forma de componentes, isso fica:

$$
v_{i}^{1} \leftarrow \frac{1}{2}\left(v_{i-1}^{1}+v_{i+1}^{0}+h^{2} f_{i}\right)
$$

Na forma matricial, fica mais claro como é o funcionamento do método de Gauss-Seidel:

$$
\begin{array}{r}
\mathbf{A}=\mathbf{D}-\mathbf{L}-\mathbf{U} \rightarrow \mathbf{A}=(\mathbf{D}-\mathbf{L})-\mathbf{U} \rightarrow \mathbf{A} \mathbf{v}=\mathbf{f} \\
(\mathbf{D}-\mathbf{L}) v-\mathbf{U} \mathbf{v}=\mathbf{f} \rightarrow(\mathbf{D}-\mathbf{L}) \mathbf{u}=\mathbf{f}+\mathbf{U} \mathbf{v} \\
\mathbf{v}=(\mathbf{D}-\mathbf{L})^{-1} \mathbf{U} \mathbf{v}+(\mathbf{D}-\mathbf{L})^{-1} \mathbf{f}
\end{array}
$$

Tomando o operador da iteração de Gauss-Seidel $\mathbf{R}_{G}$ como

$$
\mathbf{R}_{G}=(\mathbf{D}-\mathbf{L})^{-1} \mathbf{U}
$$

a iteração acima fica:

$$
\mathbf{v}=\mathbf{R}_{G} \mathbf{v}+(\mathbf{D}-\mathbf{L})^{-1} \mathbf{f}
$$

O erro desta iteração é dado por:

$$
\mathbf{e}^{1}=\mathbf{R}_{G} \mathbf{e}^{0}
$$

\footnotetext{
${ }^{8}$ Isso é equivalente a definir cada componente do resíduo como zero.
} 
Finalmente, podemos agora olhar para uma variante muito importante da iteração de GaussSeidel ${ }^{9}$, onde a única mudança é no ordenamento dos pontos do grid. Neste caso, os pontos são ordenados em RED (pontos com índices pares) e BLACK (pontos com índices ímpares) ${ }^{10}$. Esta iteração é chamada comumente de Red-Black Gauss-Seidel.

Neste caso, primeiramente são atualizadas as componentes pares pela expressão:

$$
v_{2 i} \rightarrow \frac{1}{2}\left(v_{2 i-1}+v_{2 i+1}+h^{2} f_{2 i}\right)
$$

e depois as ímpares usando:

$$
v_{2 i+1} \rightarrow \frac{1}{2}\left(v_{2 i}+v_{2 i+2}+h^{2} f_{2 i+1}\right)
$$

Na figura A.3, mostramos como fica o ordenamento dos pontos do grid, no caso $1 D$ e $2 D$ para o método Red-Black Gauss-Seidel.

(a)

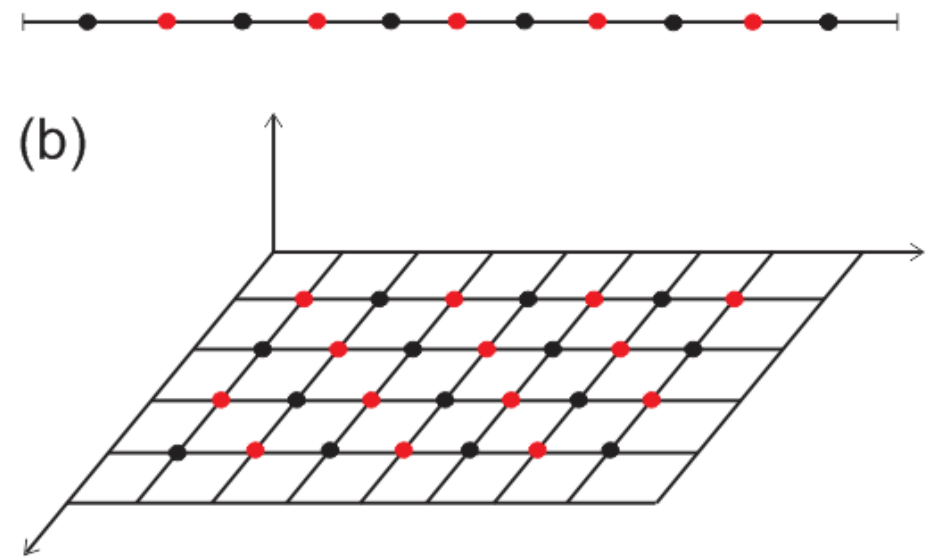

Figura A.3: Ordenamento dos pontos do grid no método Red-Black Gauss-Seidel. (a) Grid unidimensional. (b) Grid bidimensional.

\footnotetext{
${ }^{9}$ Esta variante foi a que implementamos no código SIESTA para resolução da equação de Poisson.

${ }^{10}$ Por par dizemos que a soma dos índices dos pontos do grid é par e por ímpar dizemos que a soma dos índices dos pontos do grid é ímpar.
} 


\section{A.3 Exemplos da atuação dos métodos iterativos - Jacobi e Gauss-Seidel}

Para ilustrar a atuação dos métodos iterativos vamos considerar o problema $1 D$, dado abaixo:

$$
\mathrm{Au}=0
$$

que na forma discreta fica:

$$
-u_{i-1}+2 u_{i}-u_{i+1}=0
$$

Sabemos que essa equação possui uma solução trivial $u=0$ e qualquer chute inicial que fizermos para a solução do problema, o erro da aproximação será igual ao negativo do valor do chute inicial.

Iremos utilizar os modos de Fourier do seno como uma primeira aproximação, que são dados por:

$$
\mathbf{v}_{k}=\left(v_{i}\right)_{k}=\operatorname{sen}\left(\frac{i k \pi}{N}\right)
$$

onde $i$ representa as componentes da solução em cada ponto do grid, $k$ representa o modo (ou vetor de onda) do seno e $N$ o número de pontos do grid.

Tomaremos 3 modos $k$ da aproximação $(k=1,5,10)$, pois aumentando o valor de $k$ tornamos os erros cada vez mais oscilatórios, como podemos observar na figura A.4

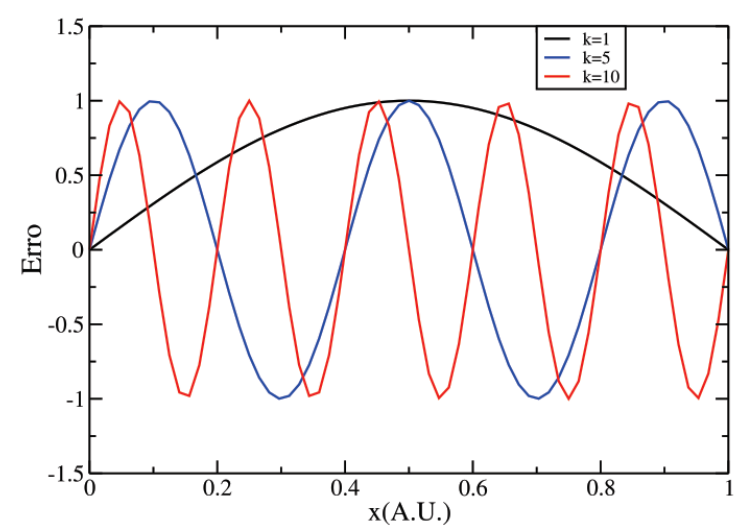

Figura A.4: Erros gerados a partir da aproximação $v_{i}=\sin \left(\frac{i k \pi}{N}\right)$ para os $\operatorname{modos} k=1,5,10$ para um grid com $N=64$ pontos. 
Essas escolhas para as aproximações (erros) irão nos ajudar a entender como é a atuação dos métodos iterativos para a solução do problema nas componentes do erro, quando esses são suaves $(k=1)$ e mais oscilatórios $(k=5,10)$. Na figura A.5, mostramos um plot da norma do erro $\left(\|e\|_{2}\right)$ em função do número de iterações, para o método de Jacobi e Gauss-Seidel.
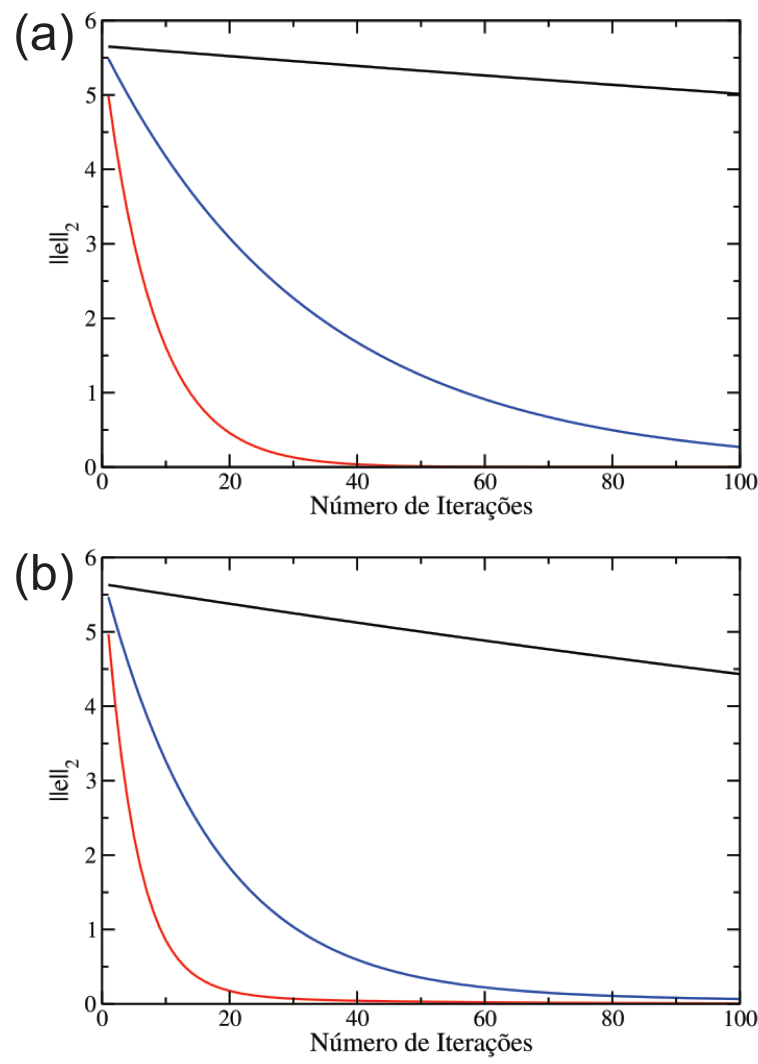

Figura A.5: Norma dos erros $\left(\|e\|_{2}\right)$ para a aproximação $v_{i}=\operatorname{sen}\left(\frac{i k \pi}{N}\right)$ para os $\operatorname{modos} k=1,5,10$ para um grid com $N=64$ pontos em função do número de iterações. (a) Método de Jacobi e (b) Método de Gauss-Seidel

Primeiramente, podemos observar que o erro fica menor a cada iteração, mostrando que ambos os métodos (Jacobi e Gauss-Seidel) servem para relaxar (reduzir) o erro da aproximação onde o método de Gauss-Seidel mostra ser mais eficiente. Agora o mais importante a ser observado é que quanto mais oscilatório for o erro, mais eficiente fica o método de relaxação, ou seja, a taxa de relaxação do erro é maior quanto mais oscilatório ele for.

Para observarmos melhor o efeito dos métodos de relaxação nas componentes do erro, vamos considerar uma nova aproximação, onde o erro seja composto de modos altamente oscilatórios e um modo mais suave. Essa nova aproximação pode ser dada por:

$$
v_{i}=\operatorname{sen}\left(\frac{2 i \pi}{N}\right)+\frac{1}{2} \operatorname{sen}\left(\frac{16 i \pi}{N}\right)+\frac{1}{2} \operatorname{sen}\left(\frac{32 i \pi}{N}\right) .
$$


Na figura A.6, mostramos qual o comportamento do erro, ao aplicarmos a iteração de Jacobi nessa aproximação: Através da observação da figura A.6, podemos concluir que os esquemas

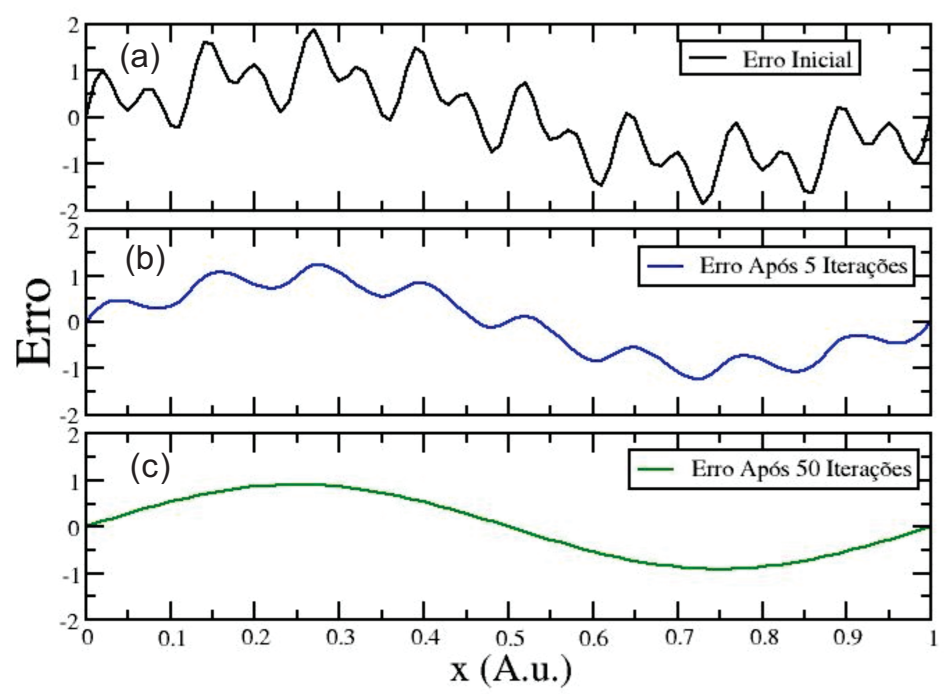

Figura A.6: (a) Erro inicial dado pela equação A.49. (b) Erro após 5 iterações. (c) Erro após 50 iterações.

iterativos de relaxação, possuem a propriedade de suavizar (eliminar) o erro, onde os modos oscilatórios são eliminados efetivamente, restando somente os mais suaves.

Existem muitos métodos de relaxação que podem ser utilizados para a construção de um algoritmo multigrid, mas usualmente, os que apresentam melhor custo benefício, são aqueles construídos a partir dos métodos de relaxação mais simples como o de Gauss-Seidel e de Jacobi.

\section{A.4 Observações antes de entrar no método de multigrid}

Antes de entrarmos de fato na descrição da técnica de multigrid, iremos fazer algumas observações, que irão nos levar de maneira intuitiva ao método de multigrid.

\section{Observação 1 Utilização de grids mais grossos - coarse grids;}

Como vimos anteriormente, os métodos de relaxação possuem a propriedade de suavização do erro, onde os modos mais oscilatórios são efetivamente eliminados, enquanto que os mais suaves são reduzidos bem vagarosamente. Isso, aparentemente, parece ser uma limitação, porém, se utilizarmos grids um pouco mais grossos ${ }^{11}$, poderemos novamente ter a vantagem da

\footnotetext{
${ }^{11}$ Esses grids ruins chamaremos de coarse grids.
} 
suavização. Para ficar mais claro, temos que responder a seguinte pergunta: Porque usar coarse grids?

\section{Razão 1 Iterações Agrupadas:}

Como o coarse grid possui menos pontos que o fine grid, podemos utilizar o primeiro para calcular um bom chute inicial para a solução no fine grid de uma maneira computacionalmente barata. Essa idéia de iterações agrupadas pode ser descrita utilizando o seguinte esquema:

- Relaxar $A u=f$ em um grid $\Omega^{4 h}$ com o chute inicial $v^{8 h}$, para obter o chute inicial $v^{2 h}$;

- Relaxar $A u=f$ em um grid $\Omega^{2 h}$ com o chute inicial $v^{4 h}$, para obter o chute inicial $v^{h}$;

- Relaxar $A u=f$ em um grid $\Omega^{h}$ com o chute inicial $v^{h}$, para obter: SOLUÇÃO FINAL!!!;

Esse esquema parece ser uma boa idéia, mas disso surgem duas questões que serão respondidas mais a frente: (1) O que significa $A u=f$ em $\Omega^{2 h}, \Omega^{4 h}, \cdots$ ? (2) E se ainda existirem componentes suaves quando chegarmos ao fine grid $\Omega^{h}$ ?

\section{Razão 2 O Erro é (relativamente) mais oscilatório no coarse grid;}

Quando estamos em um coarse grid, o erro que é suave em um fine grid aparenta ser mais oscilatório no coarse grid. Para ficar mais claro, na figura A.7(a), mostramos uma função suave, que pode ser representada através de uma interpolação linear em um coarse grid, como mostrado na figura A.7(b).

No coarse grid, o erro aparenta ser menos suave que no fine grid. Dessa maneira, a relaxação será mais efetiva se realizada no coarse grid.

\section{Observação 2 Correção do coarse grid;}

A idéia básica da correção residual, como vimos anteriormente, é: seja v uma aproximação da solução de $\mathbf{A u}=\mathbf{f}$, onde o resíduo é dado por $\mathbf{r}=\mathbf{f}-\mathbf{A v}$. O erro $\mathbf{e}=\mathbf{u}-\mathbf{v}$ também satisfaz a equação $\mathbf{A e}=\mathbf{r}$. 


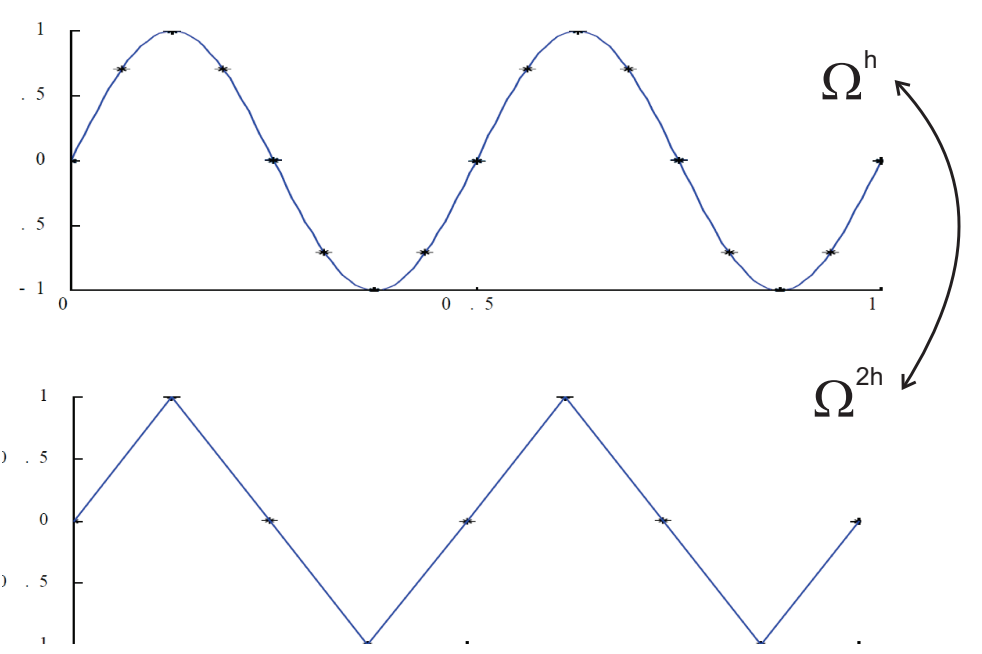

Figura A.7: Pontos do fine grid $\Omega^{h}$ mapeados para o coarse grid $\Omega^{2 h}$.

Quando relaxamos $\mathbf{A u}=\mathbf{f}$ no fine grid, o erro ficará suave. Entretanto, no coarse grid, esse erro irá parecer mais oscilatório e a sua relaxação será mais efetiva. Dessa maneira, mapeamos o problema em um coarse grid e relaxamos a equação residual $\mathbf{A e}=\mathbf{r}$, com um chute inicial para o erro de $\mathbf{e}=0$. Essa idéia pode ser resumida no esquema da correção do coarse grid.

- Relaxar $\mathbf{A u}=\mathbf{f}$ em $\Omega^{h}$ para obter a aproximação $\mathbf{v}^{h}$;

- Calcular o resíduo $\mathbf{r}=\mathbf{f}-\mathbf{A} \mathbf{v}^{h}$;

- $\operatorname{Relaxar} \mathbf{A e}=\mathbf{r}$ em $\Omega^{2 h}$ para obter uma aproximação para o erro $\mathbf{e}^{2 h}$;

- Corrigir a aproximação $\mathbf{v}^{h}$ utilizando o erro $\mathbf{e}^{2 h}: \mathbf{v}^{h} \leftarrow \mathbf{v}^{h}+\mathbf{e}^{2 h}$

Este procedimento é a base do que chamamos de esquema de correção do coarse grid. Resumidamente, o que é feito é relaxar o sistema no fine grid até a convergência deteriorar e depois é relaxar a equação residual em um coarse grid para a obtenção de uma boa aproximação para o erro. Depois disso, retorna-se para o fine grid a fim de corrigir a primeira aproximação obtida neste. O uso desses esquemas de correções envolvendo diferentes grids leva a necessidade da existência de mecanismos eficientes de transferência de informações entre esses grids, que será o objetivo da próxima seção. 


\section{A.5 Operadores de transferência entre grids}

Nesta seção iremos definir os métodos para a realização do mapeamento de informação entre os grids:

$$
\begin{aligned}
& \Omega^{h} \Rightarrow \Omega^{2 h} \\
& \Omega^{2 h} \Rightarrow \Omega^{h}
\end{aligned}
$$

Iremos considerar somente o caso em que o coarse grid possui metade dos pontos que o fine grid, ou seja, o espaçamento entre os pontos do grid é duas vezes maior no coarse grid que no fine grid. Isso é basicamente uma prática universal nos métodos de multigrid geométricos, pois não existe nenhuma vantagem aparente na utilização de grids com razões diferentes de 2 .

\section{A.5.1 Operadores de interpolação}

Operadores que levam informação do coarse grid para o fine grid são comumente chamados de operadores de interpolação ou prolongação. Iremos considerar somente o operador de interpolação linear, pois no caso de um multigrid geométrico este mostra ser mais eficiente.

\section{Operador de interpolação linear}

Os operadores de interpolação linear em $d$ dimensões podem facilmente ser obtidos através de um procedimento recursivo (sobre as $d$ dimensões) a partir do operador de interpolação $1 D$. Dessa maneira, iremos tratar primeiramente o caso $1 D$ para depois obtermos em outras dimensões.

Seja o seguinte mapeamento:

$$
\mathbf{I}_{2 h}^{h}: \Omega^{2 h} \rightarrow \Omega^{h}
$$

onde $\mathbf{v}^{h}$ e $\mathbf{v}^{2 h}$ são definidos em $\Omega^{h}$ e $\Omega^{2 h}$, respectivamente. Assim, o mapeamento pode ser escrito como:

$$
\mathbf{I}_{2 h}^{h} \mathbf{v}^{2 h}=\mathbf{v}^{h}
$$


onde na forma de componentes esse processo pode ser visto como:

$$
\begin{array}{r}
v_{2 i}^{h}=v_{i}^{2 h} \\
v_{2 i+1}^{h}=\frac{1}{2}\left(v_{i}^{2 h}+v_{i+1}^{2 h}\right)
\end{array}
$$

A figura A.8 mostra graficamente a atuação de $\mathbf{I}_{2 h}^{h}$. Uma vez definido o processo de interpo-

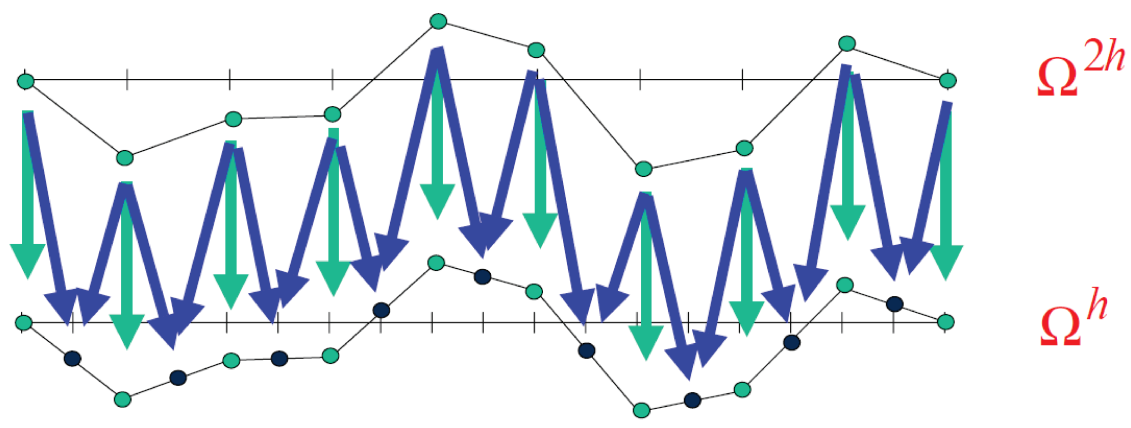

Figura A.8: Os valores em pontos do coarse grid, são mapeados para o fine grid sem nenhuma modificação. Os demais valores dos pontos no fine grid que não são pontos do coarse grid, são obtidos através de médias entre os vizinhos dos pontos no coarse grid.

lação, temos que saber o seguinte: Esse processo de interpolação é eficiente e funciona bem?

Para responder isso, primeiramente vamos assumir que o erro real ${ }^{12}$ é um vetor suave no fine grid. Assumiremos também que a aproximação do coarse grid para o erro foi determinada em $\Omega^{2 h}$ e que ela é exata nos pontos do coarse grid. Quando essa aproximação no coarse grid é interpolada para o fine grid, o resultado final também será suave. Consequentemente, é esperado uma aproximação relativamente boa para o erro no fine grid, como ilustrado na figura A.9.

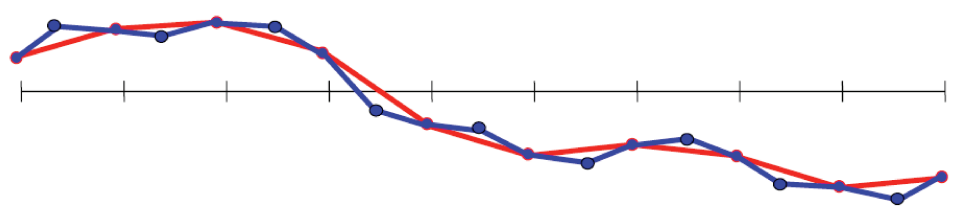

Figura A.9: Se o erro exato em $\Omega^{h}$ (Representado pela linha vermelha) é suave, o erro obtido da interpolação do coarse grid $e^{2 h}$ (linha azul) deve fornecer uma boa representação do erro exato no fine grid.

Em contraste, se o erro real for oscilatório, mesmo uma boa aproximação no coarse grid,

\footnotetext{
${ }^{12}$ Que não é conhecido exatamente.
} 
irá produzir um resultado que não é muito preciso. Essa situação é mostrada na figura A.10. Assim, a interpolação é mais eficiente quando o erro é suave tanto no coarse grid quanto no

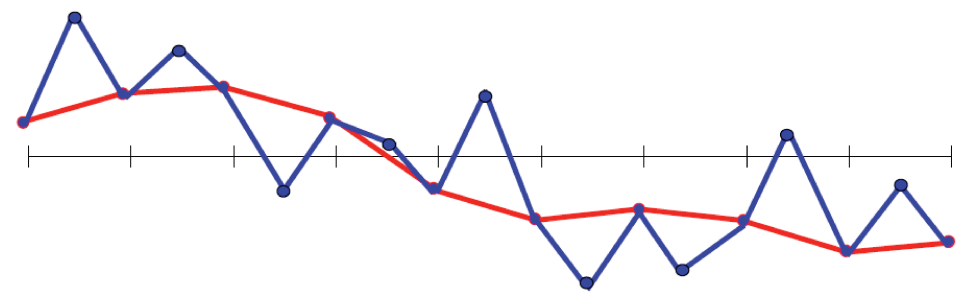

Figura A.10: Se o erro exato em $\Omega^{h}$ (indicado pela linha vermelha) for oscilatório, o resultado do erro obtido a partir da interpolação no coarse grid ( indicado por azul) não irá fornecer uma boa aproximação para o erro no fine grid.

fine grid.

Devido o processo de interpolação ser necessário nos processos das iterações agrupadas e no esquema de correção do coarse grid, podemos concluir que esses dois processos, são mais eficientes quando o erro da aproximação é suave. Como veremos em breve, esses processos fornecem um complemento aos processos de relaxação, que como já vimos, são mais efetivos quando o erro é oscilatório.

\section{Operador de interpolação linear em $2 D$}

Para o problema $2 D$, o operador de interpolação pode ser definido de uma maneira similar que o problema $1 D$. Tomando o problema do mapeamento $2 D$ como $\mathbf{I}_{2 h}^{h} \mathbf{v}^{2 h}=\mathbf{v}^{h}$, na forma de componentes isso pode ser escrito como:

$$
\begin{array}{r}
v_{2 i, 2 j}^{h}=v_{i j}^{2 h} \\
v_{2 i+1,2 j}^{h}=\frac{1}{2}\left(v_{i j}^{2 h}+v_{i+1, j}^{2 h}\right) \\
v_{2 i, 2 j+1}^{h}=\frac{1}{2}\left(v_{i j}^{2 h}+v_{i, j+1}^{2 h}\right) \\
v_{2 i+1,2 j+1}^{h}=\frac{1}{4}\left(v_{i j}^{2 h}+v_{i+1, j}^{2 h}+v_{i, j+1}^{2 h}+v_{i+1, j+1}^{2 h}\right)
\end{array}
$$

\section{A.5.2 Operadores de restrição}

A segunda classe de operadores de transferência entre grids, envolvem a movimentação de vetores no fine grid para o coarse grid. Eles são geralmente chamados de operadores de restrição, e são denotados por $\mathbf{I}_{h}^{2 h}$. O operador mais óbvio é o de injeção. Ele é definido como 
$\mathbf{I}_{h}^{2 h} \mathbf{v}^{h}=\mathbf{v}^{2 h}$, onde na forma de componentes pode ser escrito como

$$
v_{j}^{2 h}=v_{2 j}^{h}
$$

Em outras palavras, com a injeção, o vetor no coarse grid simplesmente toma os valores diretamente do ponto correspondente no fine grid.

Um operador de restrição alternativo é o chamado full weighting, definido como $\mathbf{I}_{h}^{2 h} \mathbf{v}^{h}=$ $\mathbf{v}^{2 h}$, onde na forma de componentes para o caso $1 D$ é dado por:

$$
v_{j}^{2 h}=\frac{1}{4}\left(v_{2 j-1}^{h}+2 v_{j}^{h}+v_{2 j+1}^{h}\right) .
$$

Neste caso, os valores no vetor do coarse grid são médias ponderadas dos valores vizinhos no fine grid.

O operador de full weighting em duas dimensões é dado na forma de componentes por:

$$
\begin{aligned}
v_{i j}^{2 h} & =\frac{1}{16}\left[v_{2 i-1,2 j-1}^{h}+v_{2 i+1,2 j-1}^{h}+v_{2 i-1,2 j+1}^{h}+v_{2 i+1,2 j+1}^{h}\right. \\
& +2\left(v_{2 i, 2 j-1}^{h}+v_{2 i, 2 j+1}^{h}+v_{2 i+1,2 j}^{h}+v_{2 i-1,2 j}^{h}\right) \\
& \left.+4 v_{2 i, 2 j}^{h}\right]
\end{aligned}
$$

Agora já temos de maneira bem definida as maneiras de como são feitas as transferências entre vetores no fine grid com o coarse grid. Consequentemente, podemos retornar ao esquema de correção e descrever este de maneira precisa. Para fazer isso, primeiramente iremos descrever um esquema de correção de dois grid.

\section{A.6 Esquema de correção de dois grids - $\mathbf{v}^{h} \leftarrow M G\left(\mathbf{v}^{h}, \mathbf{f}^{h}\right)$.}

O esquema de correção de dois grids pode ser descrito a partir da seguinte seqüencia de operações:

- Relaxa-se $\nu_{1}$ vezes o problema $\mathbf{A}^{h} \mathbf{u}^{h}=\mathbf{f}^{h}$ em $\Omega^{h}$ com o chute inicial $\mathbf{v}^{h}$;

- Calcula-se o resíduo no fine grid $\mathbf{r}^{h}=\mathbf{f}^{h}-\mathbf{A}^{h} \mathbf{v}^{h}$;

- Restringe o resíduo do fine grid para o coarse grid: $\mathbf{r}^{2 h}=I_{h}^{2 h} \mathbf{r}^{2 h}$;

- Resolver-se $\mathbf{A}^{2 h} \mathbf{e}^{2 h}=\mathbf{r}^{2 h}$ em $\Omega^{2 h}$; 
- Interpola-se o erro no coarse grid para o fine grid por $\mathbf{e}^{2 h}=I_{2 h}^{h} \mathbf{e}^{2 h}$;

- Corrige-se a aproximação no fine grid por $\mathbf{v}^{h} \leftarrow \mathbf{v}^{h}+\mathbf{e}^{h}$;

- Relaxa-se $\nu_{2}$ vezes o problema $\mathbf{A}^{h} \mathbf{u}^{h}=\mathbf{f}^{h}$ em $\Omega^{h}$ com o novo chute inicial $\mathbf{v}^{h}$;

O procedimento descrito anteriormente é simplesmente o esquema de correção original refinado pelo uso dos operadores de transferência entre grids. Existem alguns comentários importantes a ser feito sobre esse esquema. Primeiramente, note que os índices $h$ e $2 h$ são essencialmente para indicar o grid em que uma aproximação em particular está sendo definida. Segundo, todas as quantidades no procedimento anterior são bem definidas exceto por $\mathbf{A}^{2 h}$. Para o nosso problema, esse operador é definido a partir da discretização do problema no grid $\Omega^{2 h}$. Finalmente, os inteiros $\nu_{1}$ e $\nu_{2}$ são parâmetros no esquema que controlam o número de passos de relaxação antes e depois da visitação do coarse grid, onde usualmente são parâmetros de entrada e se mantém fixos durante o multigrid.

É importante observarmos a complementaridade dos intens que estão relacionados no processo acima. A relaxação no fine grid elimina as componentes oscilatórias do erro, deixando relativamente somente as componentes suaves do erro. Assumindo que a equação residual pode ser resolvida de maneira acurada em $\Omega^{2 h}$, ainda é importante de transferir o erro de maneira acurada de volta para o fine grid. Como o erro é suave, a interpolação irá funcionar muito bem e a correção no fine grid da solução deverá ser muito efetiva.

O esquema de correção de dois grids, como descrito anteriormente, deixa uma questão ainda em aberto com respeito ao procedimento: Qual é a melhor maneira de resolver o problema do coarse grid $\mathbf{A}^{2 h} \mathbf{e}^{2 h}=\mathbf{r}^{2 h}$ ? A resposta pode ser algo aparente, particularmente quando se pensa recursivamente. O problema no coarse grid, não é muito diferente do problema original no fine grid. Consequentialness, podemos aplicar novamente o esquema de correção de dois grids para a equação residual em $\Omega^{2 h}$, que significa que iremos relaxar o erro lá, e então mover esse para um outro coarse grid $\Omega^{4 h}$, para o próximo passo do esquema de correção. Esse processo pode ser repetido em sucessivos coarse grids até uma solução direta da equação residual ser possível $^{13}$.

\footnotetext{
${ }^{13}$ Por exemplo, se reduzirmos o problema a um coarse grid de 3 pontos onde 2 desses são contornos fixos, o outro ponto restante pode ser resolvido exatamente.
} 
Para facilitar a descrição desse procedimento, utilizaremos a seguinte notação. $O$ vetor que define o lado direito da equação da equação residual será agora $\mathbf{f}^{2 h}$ no lugar de $\mathbf{r}^{2 h}$, pois nesse nível ele representa novamente o lado direito de uma equação. No lugar de chamar a solução da equação residual de $\mathbf{e}^{2 h}$, iremos usar $\mathbf{u}^{2 h}$, pois simplesmente ele representa um novo vetor de solução. Podemos utilizar também $\mathbf{v}^{2 h}$ para identificar uma aproximação para $\mathbf{u}^{2 h}$. Essas mudanças simplificam muito a notação facilitando a visualização do processo recursivo, mas é importante ainda ter em mente o significado de cada uma dessas variáveis em cada nível do grid.

\section{A.7 Ciclos Multigrid}

Vários ciclos podem ser construídos utilizando-se o esquema de correção de dois grids de maneira sucessiva. Dentre esses ciclos, existem os $V, W, \mu$, ciclos full-multigrid(FMG), dentre muitos outros. Assim, dedicaremos a próxima seção aos ciclos que foram implementados no código SIESTA - Ciclos $V$ e $F M G^{14}$.

\section{A.7.1 Ciclo $V-\mathbf{v}^{h} \leftarrow V^{h}\left(\mathbf{v}^{h}, \mathbf{f}^{h}\right)$.}

O algoritmo do ciclo $V$ pode ser descrito seguindo os seguintes passos:

- Relaxa-se $\mathbf{A}^{h} \mathbf{u}^{h}=\mathbf{f}^{h} \nu_{1}$ vezes com o chute inicial $\mathbf{v}^{h}$;

- Calcula-se $\mathbf{f}^{2 h}=I_{h}^{2 h} \mathbf{r}^{h}$;

- Relaxa-se $\mathbf{A}^{2 h} \mathbf{u}^{2 h}=\mathbf{f}^{2 h} \nu_{1}$ vezes com o chute inicial $\mathbf{v}^{2 h}=0$;

- Calcula-se $\mathbf{f}^{4 h}=I_{2 h}^{4 h} \mathbf{r}^{2 h}$

* Relaxa-se $\mathbf{A}^{4 h} \mathbf{u}^{4 h}=\mathbf{f}^{4 h} \nu_{1}$ vezes com o chute inicial $\mathbf{v}^{4 h}=0$;

* Calcula-se $\mathbf{f}^{8 h}=I_{4 h}^{8 h} \mathbf{r}^{4 h}$;

- Resolve-se $\mathbf{A}^{L h} \mathbf{u}^{L h}=\mathbf{f}^{L h}$;

. :

$*$ Corrige-se $\mathbf{v}^{4 h} \leftarrow \mathbf{v}^{4 h}+I_{8 h}^{4 h} \mathbf{v}^{8 h} ;$

\footnotetext{
${ }^{14}$ Foi implementado uma outra variação do ciclo $V$ conhecido como ciclo $W$.
} 
* Relaxa-se $\mathbf{A}^{4 h} \mathbf{u}^{4 h}=\mathbf{f}^{4 h} \nu_{2}$ vezes com o chute inicial $\mathbf{v}^{4 h}$;

- Corrige-se $\mathbf{v}^{2 h} \leftarrow \mathbf{v}^{2 h}+I_{4 h}^{2 h} \mathbf{v}^{4 h}$

- Relaxa-se $\mathbf{A}^{2 h} \mathbf{u}^{2 h}=\mathbf{f}^{2 h} \nu_{2}$ vezes com o chute inicial $\mathbf{v}^{2 h}$;

- Corrige-se $\mathbf{v}^{h} \leftarrow \mathbf{v}^{h}+I_{2 h}^{h} \mathbf{v}^{2 h}$;

- Relaxa-se $\mathbf{A}^{h} \mathbf{u}^{h}=\mathbf{f}^{h} \nu_{2}$ vezes com o chute inicial $\mathbf{v}^{h}$;

O ciclo $V$ pode ser escrito de uma forma mais compacta na forma de diagramas, como na figura A.11.

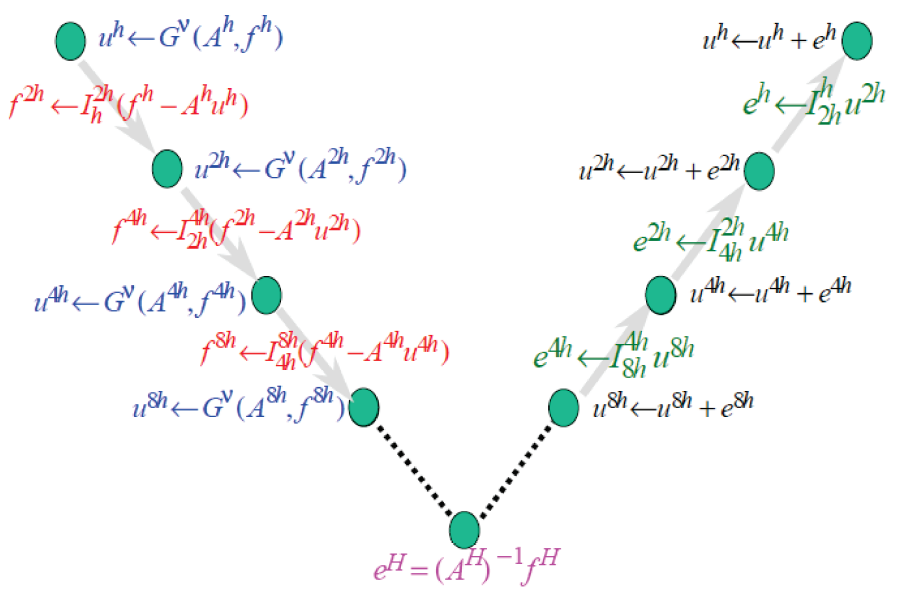

Figura A.11: Esquema de um ciclo $V$.

Como observamos na figura, esse algoritmo desce até o coarse grid, que pode consistir de um ou mais pontos interiores do grid, e depois sobe de volta ao fine grid. A figuraA.11 mostra a ordem em que os grids são visitados. Devido a forma do diagrama, esse algoritmo é chamado de ciclo $V .^{15}$

\section{A.7.2 Ciclo full-multigrid $-\mathbf{v}^{h} \leftarrow F M G^{h}\left(f^{h}\right)$}

O algoritmo do ciclo $F M G$ pode ser descrito seguindo os seguintes passos:

- Inicializa-se $\mathbf{f}^{2 h} \leftarrow I_{h}^{2 h} \mathbf{f}^{h}, \mathbf{f}^{4 h} \leftarrow I_{2 h}^{4 h} \mathbf{f}^{2 h}, \cdots$

- $\quad * \quad$ Resolve-se ou relaxa-se no menor coarse grid, $\Omega^{L h}$;

:

\footnotetext{
${ }^{15}$ Esse ciclo é uma das possíveis escolhas para a solução da equação de Poisson em nossa implementação.
} 
$* \mathbf{v}^{4 h} \leftarrow I_{8 h}^{4 h} \mathbf{v}^{8 h}$

$* \mathbf{v}^{4 h} \leftarrow V^{4 h}\left(\mathbf{v}^{4 h}, \mathbf{v}^{4 h}\right), \mu_{0}$ vezes;

$-\mathbf{v}^{2 h} \leftarrow I_{4 h}^{2 h} \mathbf{v}^{4 h}$

$\mathbf{-} \mathbf{v}^{2 h} \leftarrow V^{2 h}\left(\mathbf{v}^{2 h}, \mathbf{v}^{2 h}\right), \mu_{0}$ vezes;

- $\mathbf{v}^{h} \leftarrow I_{2 h}^{h} \mathbf{v}^{2 h}$

- $\mathbf{v}^{h} \leftarrow V^{h}\left(\mathbf{v}^{h}, \mathbf{v}^{h}\right), \mu_{0}$ vezes;

O ciclo FMG pode ser escrito de uma forma mais compacta utilizando diagramas, como na figura A.12.

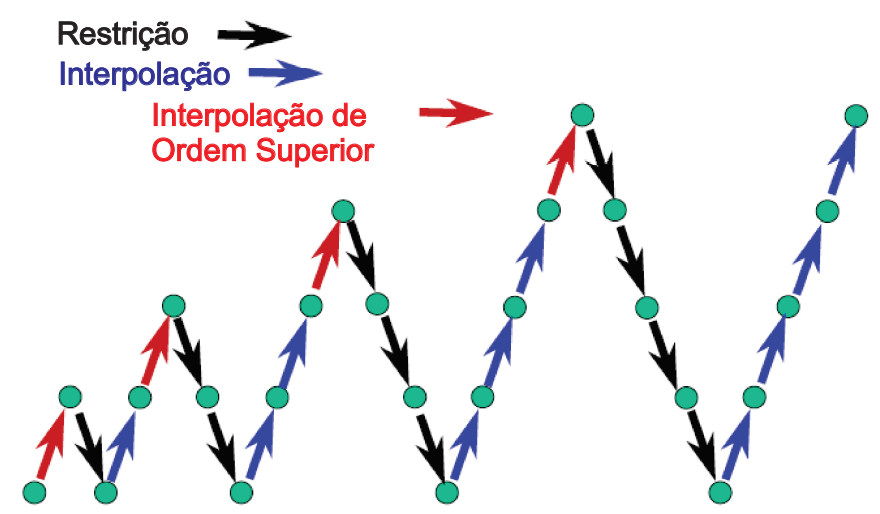

Figura A.12: Esquema de um ciclo $F M G$.

Este algoritmo inicia-se definindo o lado direito da equação para todos os coarse grids, transferindo-se $\mathbf{f}^{h}$ a partir do fine grid. Uma outra opção é usar o lado direito original da equação $f$ para os pontos correspondentes. O parâmetro $\mu_{0}$ determina o número de ciclos $V$ que são feitos em cada nível dos coarse grids.

O ciclo FMG é uma síntese notável das idéias e técnicas que, individualmente são bem conhecidas e vem sendo utilizadas por um longo tempo. Tais técnicas tomadas isoladamente, apresentam alguns defeitos. O FMG é a técnica que serve para integrá-las para que possam trabalhar em conjunto de uma maneira que supera todas essas limitações. O resultado final é um algoritmo muito poderoso. 


\section{Apêndice B}

\section{Lista de Publicações}

1- Padilha, J. E.; Pontes, R. B. ; da Silva, A. J. R. ; Fazzio, A.

IxV curves of boron and nitrogen doping zigzag graphene nanoribbons.

International Journal of Quantum Chemistry, v. 111, p. 1379-1386, 2011.

2- Padilha, J. E. ; Lima, M. P. ; da Silva, A. J. R. ; Fazzio, A.

Bilayer graphene dual-gate nanodevice: An ab initio simulation.

Physical Review. B, Condensed Matter and Materials Physics, v. 84, p. 113412, 2011.

3- Padilha, J. E. ; Pontes, R. B.; Fazzio, A.

Bilayer graphene on h-BN substrate: investigating the breakdown voltage and tuning the bandgap by electric field.

Journal of Physics: Condensed Matter, v. 24, p. 075301, 2012. 


\section{Referências Bibliográficas}

[1] Geim, A. K. and Novoselov, K. S. Nature Materials 6, 183 (2007).

[2] Avouris, P. Nano Lett. 10 (2010).

[3] Farmer, D. B., Chiu, H.-Y., Lin, U.-M., Jenkins, K., Xia, F., and Avouris, P. Nano Lett. 9, 4474 (2009).

[4] Zhu, W., Perebeinos, V., Freitag, M., and Avouris, P. Phys. Rev. B 80, 235402 (2009).

[5] Neto, A. H. C., Guinea, F., Peres, N. M. R., Novoselov, K. S., and Geim, A. K. Rev. Mod. Phys. 81, 109 (2009).

[6] Matsubara, T. Prog. Teor. Phys. 14, 351 (1955).

[7] Weitz, R. T., Allen, M. T., Feldman, B. E., Martin, J., and Yacoby, A. Science 330, 812 (2010).

[8] Zhang, Y., Tang, T.-T., Girit, C., Hao, Z., Martin, M. C., Zettl, A., Crommie, M. F., Shen, Y. R., and Wang, F. Nature 459, 820 (2009).

[9] Decker, R., Wang, Y., Brar, V. W., Regan, W., Tsai, H.-Z., Wu, Q., Gannett, W., Zettl, A., and Crommie, M. F. Nano Lett. 11, 2291 (2011).

[10] Bae, S., Kim, H., Lee, Y., Xu, X., Park, J.-S., Zheng, Y., Balakrishnan, J., Lei, T., Kim, H. R., Song, Y. I., Kim, Y.-J., Kim, K. S., Özyilmaz, B., Ahn, J.-H., Hong, B. H., and Iijima, S. Nat. Nanotech. 5, 574 (2010).

[11] Ohta, T., Bostwick, A., Seyller, T., Horn, K., and Rotenberg, E. Science 313, 951 (2010).

[12] Wolf, S. A., Awschalom, D. D., Buhrman, R. A., Daughton, J. M., von Molnár, S., Roukes, M. L., Chtchelkanova, A. Y., and Treger, D. M. Science 294, 1488 (2001). 
[13] Parkina, S. S. P., Roche, K. P., Samant, M. G., Rice, P. M., Beyers, R. B., Scheuerlein, R. E., O’Sullivan, E. J., Brown, S. L., Bucchigano, J., Abraham, D. W., Lu, Y., Rooks, M., Trouilloud, P. L., Wanner, R. A., and Gallagher, W. J. J. Appl. Phys. 85, 5828 (1999).

[14] M. N. Baibich, J. M. Broto, A. F. F. N. V. D., F. Petroff P. Etienne, G. Creuzet, A. F., and Chazelas, J. Phys. Rev. Lett. 61, 2472 (1988).

[15] Kroto, H. W., Heath, J. R., O’Brien, S. C., Curl, R. F., and Smalley, R. E. Nature 318, $162(1985)$.

[16] Iijima, S. Nature 56, 354 (1991).

[17] Wallace, P. R. Phys. Rev. 71, 622 (1947).

[18] Semenoff, G. W. Phys. Rev. Lett. 53, 2449 (1984).

[19] Haldane, F. D. M. Phys. Rev. Lett. 61, 2015 (1988).

[20] Ajiki, H. and Ando, T. J. Phys. Soc. Japan 62, 1255 (1993).

[21] Mermin, N. D. and Wagner, H. Phys. Rev. Lett. 17, 1133 (1966).

[22] Fasolino, A., Los, J. H., and Katsnelson, M. I. Nat. Mater. 6, 858 (2007).

[23] Novoselov, K. S., Geim, A. K., Morozov, S. V., Jiang, D., Zhang, Y., Dubonos, S. V., Grigorieva, I. V., and Firsov, A. A. Science 306, 666 (2004).

[24] Zhang, Y., Tan, Y. W., Stormer, H. L., and Kim, P. Nature 438, 201 (2005).

[25] Bolotin, K. I., Sikes, K. J., Jiang, Z., Klima, M., Fudenberg, G., Hone, J., Kima, P., and Stormer, H. Solid State Commun. 149, 1080 (2009).

[26] Du, X., Skachko, I., Barker, A., and Andrei, E. Y. Nat. Nanotech. 3, 491 (2008).

[27] Zhu, W., Perebeinos, V., Freitag, M., and Avouris, P. Phys. Rev. B 80, 235420 (2009).

[28] Chen, J.-H., Jang, C., Xiao, S., Ishigami, M., and Fuhrer, M. S. Nat. Nanotech. 3, 206 (2008). 
[29] Dean, C. R., Young, A. F., Meric, I., Lee, C., Wang, L., Sorgenfrei, S., Watanabe, K., Taniguchi, T., Kim, P., Shepard, K. L., and Hone, J. Nat. Nanotech. 5, 722 (2010).

[30] Dimitrakopoulos, C. J. Vac. Sci. Technol. B 28, 985 (2010).

[31] Lin, Y.-M., Dimitrakopoulos, C., Jenkins, K. A., Farmer, D. B., Chiu, H.-Y., Grill, A., and Avouris, P. Science 327, 662 (2010).

[32] Chen, Z., Lin, Y.-M., and Avouris, P. Physica E 40, 228 (2007).

[33] Han, M. Y., Özyilmaz, B., and amd P. Kim, Y. Z. Phys. Rev. Lett. 98, 206805 (2007).

[34] Lin, Y.-M., Perebeinos, V., Chen, Z., and Avouris, P. Phys. Rev. B 8, 161409 (2008).

[35] Li, X. L., Wang, X. R., Zhang, L., Lee, S. W., and Dai, H. J. Science 319, 1229 (2008).

[36] Kosynkin, D. V., Higginbotham, A. L., Sinitskii, A., Lomeda, J. R., Dimiev, A., Price, B. K., and Tour, J. M. Nature 458, 872 (2009).

[37] Cai, J., Ruffieux, P., Jaafar, R., Bieri, M., Braun, T., Blankenburg, S., Muoth, M., Seitsonen, A. P., Saleh, M., Feng, X., Müllen, K., and Fasel, R. Nature 466, 470 (2010).

[38] Koshino, M. Phys. Rev. B 81, 125304 (2010).

[39] Koshino, M. and McCann, E. Phys. Rev. B 81, 12544 (2010).

[40] McCann, E. Phys. Rev. B 74, 161403(R) (2006).

[41] Hohenberg, P. and Kohn, W. Phys. Rev. 136, B864 (1964).

[42] Parr, R. and Yang, W. Density Functional Theory of Atoms and Molecules. Oxford University Press, (1994).

[43] Viana, J. D. M., Fazzio, A., and Canuto, S. Teoria Quântica de Moléculas e Sólidos. Simulação Computacional. Editora Livraria da Física, São Paulo, (2004).

[44] Kohn, W. and Sham, L. J. Phys. Rev. 140, A1133 (1965).

[45] Schwinger, J. J. Math. Phys. 2, 407 (1961). 
[46] Keldysh, L. V. Soviet Phys. JETP 20, 1018 (1965).

[47] Caroli, C., Combescot, R., Nozieres, P., and Saint-James, D. J. Phys. C 4, 916 (1971).

[48] Datta, S. Quantum Transport: Atom to Transistor. Cambridge University Press, Cambridge, (2005).

[49] Ventra, M. D., Pantelides, S. T., and Lang, N. D. Phys. Rev. Lett. 84, 979 (2000).

[50] Ventra, M. D., Kim, S. G., Pantelides, S. T., and Lang, N. D. Phys Rev. B 86, 288 (2001).

[51] Ventra, M. D., Pantelides, S. T., and Lang, N. D. Appl. Phys. Lett 76, 3448 (2000).

[52] Haug, H. and Jauho, A. P. Quantum Kinetics in Transport and Optics of Semiconductors. Springer-Verlag, Berlin, (1996).

[53] Derosa, P. A. and Seminario, J. M. J. Phys. Chem. B 105, 471 (2001).

[54] Kondo, H., Kino, H., Nara, J., Ozaki, T., and Ohno, T. Phys. Rev. B 73, 235323 (2006).

[55] Wei, Y., Xu, Y., Wang, J., and Guo, H. Phys. Rev. B 70, 193406 (2004).

[56] Grigoriev, A., Skorodumova, N., Simak, S. I., Wendin, G., Johansson, B., and Ahuja, R. Phys. Rev. Lett. 97, 236807 (2006).

[57] Z. Li, H. Qian, J. W. B.-L. G. and Duan, W. Phys. Rev. Lett. 100, 408 (2008).

[58] Kim, W. Y. and Kim, K. S. Nature Nanotechnology 3, 408 (2008).

[59] Novaes, F. D., da Silva, A. J. R., and Fazzio, A. Braz. J. Phys. 35, 799 (2001).

[60] Sancho, M. P. L., Sancho, J. M. L., Sancho, J. M. L., , and Rubio, J. J. Phys. F 15, 851 (1985).

[61] Meir, Y. and Wingreen, N. S. Phys. Rev. Lett 68, 2512 (1992).

[62] Ostinaga, J. B., Heersche, H. B., Liu, X., Morpurgo, A. F., and Vandersypen, L. M. K. Nature Mat. 7, 151 (2007).

[63] Mak, K. F., Lui, C. H., Shan, J., and Heinz, T. F. Phys. Rev. Lett. 102, 256405 (2009). 
[64] Capelle, K. Braz. J. Phys. 36, 1318 (2006).

[65] Landauer, R. L. Philos. Mag. 21, 863 (1970).

[66] Büttiker, M., Ymry, Y., Landauer, R., and Pinhas, S. Phys. Rev. B 31, 6207 (1985).

[67] Büttiker, M. Phys. Rev. Lett. 57, 1761 (1986).

[68] Merrick, M. P., Yuer, K. A., and Beck, T. L. J. Phys. Chem. 99, 12478 (1995).

[69] Ceperley, D. M. and Alder, B. J. Phys. Rev. Lett. 45, 566 (1980).

[70] Grimme, S. J. Comp. Chem. 27 (2006).

[71] Min, H. K., Sahu, B., Banerjee, S. K., and MacDonald, A. H. Phys. Rev. B 75, 155115 (2007).

[72] Lima, M. P., Fazzio, A., and da Silva, A. J. R. Phys. Rev. B 79, 153401 (2009).

[73] Monkhorst, H. J. and Pack, J. D. Phys. Rev. B 13, 5188 (1976).

[74] Xia, F., Farmer, D. B., Lin, Y. M., and Avouris, P. Nano Lett. 10(2), 715 (2010).

[75] Taychatanapat, T. and Jarillo-Herrero, P. Phys. Rev. Lett. 105, 166601 (2010).

[76] Kuzmenko, A. B., Crassee, I., van der Marel, D., Blake, P., and Novoselov, K. S. Phys. Rev. B 80, 165406 (2009).

[77] Morozov, S. V., Novoselov, K. S., Katsnelson, M. I., Schedin, F., Elias, D. C., Jaszczak, J. A., and Geim, A. K. Phys. Rev. Lett. 100, 016602 (2008).

[78] Latil, S. and Henrard, L. Phys. Rev. Lett. 97, 036803 (2006).

[79] Aoki, M. and Amawashi, H. Solid State Commun. 142, 123 (2007).

[80] Min, H. and MacDonald, A. H. Phys. Rev. B 77, 155416 (2008).

[81] Guinea, F., Neto, A. H. C., and Peres, N. M. R. Phys. Rev. B 73, 245426 (2006).

[82] Lipson, H. and Stokes, A. R. Proc. R. Soc. A 181, 101 (1942). 
[83] Partoens, B. and Peeters, F. M. Phys. Rev. B 75, 193402 (2007).

[84] Zhang, F., Sahu, B., Min, H., and MacDonald, A. H. Phys. Rev. B 82, 035409 (2010).

[85] Mak, K. F., Shan, J., and Heinz, T. F. Phys. Rev. Lett. 104, 176404 (2010).

[86] Taychatanapat, T., Watanabe, K., Taniguchi, T., and J.-Herrero, P. Nature Physics 7, 621 (2011).

[87] Liu, Y., Lew, W. S., Goolaup, S., Liew, H. F., Wong, S. K., and Zhou, T. ACS Nano 5(7), $5490(2011)$

[88] Lui, C. H., Li, Z., Chen, Z., Klimov, P. V., Brus, L. E., and Heinz, T. F. Nano Lett. 11, $164(2011)$.

[89] Craciun, M. F., Russo, S., Yamamoto, M., Oostinga, J. B., Morpurgo, A. F., and Tarucha, S. Nat. Nanotech. 4, 383 (2009).

[90] Cousins, C. S. G. Phys. Rev. B 67, 024110 (2003).

[91] Charlier, J.-C., Gonze, X., and Michenaud, J.-P. Carbon 32, 289 (1994).

[92] Soler, J. M., Artacho, E., Gale, J. D., García, A., Junquera, J., Ordejón, P., and SanchézPortal, D. J. Phys. Condens. Matter. 14, 2745 (2002).

[93] Perdew, J. P., Burke, K., and M. Phys. Rev. Lett. 77, 3865 (1996).

[94] Schabel, M. C. and Martins, J. L. Phys. Rev. B 46, 7185 (1992).

[95] Hao, Y., Wang, Y., Wang, L., Ni, Z., Wang, Z., Wang, R., Koo, C. K., Shen, Z., and Thong, J. T. L. Small 6, 195 (2010).

[96] Ando, T. J. Phys. Soc. Jpn. 75, 074716 (2006).

[97] Nomura, K. and MacDonald, A. H. Phys. Rev. Lett. 98, 076602 (2007).

[98] Hwang, E. H., Adams, S., and Sarma, S. D. Phys. Rev. Lett. 98, 186806 (2007). 
[99] Ponomarenko, L. A., Yang, R., Mohiuddin, T. M., Katsnelson, M. I., Novoselov, K. S., Morozov, S. V., Zhukov, A. A., Schedin, F., Hill, E. W., and Geim, A. K. Phys. Rev. Lett. 102, 206603 (2009).

[100] Lafkioti, M., Krauss, B., Lohmann, T., Zschieschang, T., Klauk, H., v. Klitzing, K., and Smet, J. H. Nano Lett. 10, 1149 (2010).

[101] Liao, L., Bai, J., Qu, Y., Huang, Y., and Duan, X. Nanotechnology 21, 015805 (2010).

[102] Watanabe, K., Tanigushi, T., and Kanda, H. Nat. Mater. 3, 404 (2004).

[103] Giovannetti, G., Khomyakov, P., Brocks, G., Kelly, P., and Brink, J. V. D. Phys. Rev. B 76, 073103 (2007).

[104] Lui, C. H., Liu, L., Mak, K. F., Flynn, G. W., and Heinz, T. F. Nature 462, 339 (2009).

[105] Young, A. F., Dean, C. R., Meric, I., Sorgenfrei, S., Ren, H., Watanabe, K., Taniguchi, T., Hone, J., Shepard, K. L., and Kim, P. preprint at arxiv, 1004.5556v2 (2010).

[106] Meric, I., Han, M. Y., Young, A. F., Ozyilmaz, B., Kim, P., and Shepard, K. L. Nat. Nanotech. (2008).

[107] Schwierz, F. Nature Nanotech. 5, 487 (2010).

[108] Warner, J. H., Rümmeli, M. H., Bachmatiuk, A., and Büchner, B. ACS Nano 4, 1299 (2010).

[109] Marom, N., Bernstein, J., Garel, J., Tkatchenko, A., Joselevich, E., Kronik, L., and Hod, O. Phys. Rev. Lett. 105, 046801 (2010).

[110] Novoselov, K. S., Jiang, D., Schedin, F., Booth, T. J., Khotkevich, V. V., Morozov, S. V., and Geim, A. K. Proc. Natl. Acad. Sci. USA 102, 10451 (2005).

[111] Han, W. Q., Wu, L., Zhu, Y., Watanabe, K., and Taniguchi, T. Appl. Phys. Lett. 93, 223103 (2008).

[112] Jin, C., Lin, F., Suenaga, K., and Iijima, S. Phys. Rev. Lett. 102, 195505 (2009). 
[113] Meyer, J. C., Chivilin, A., Algara-Siller, G., Biskupek, J., and Kaiser, U. Nano Lett. 9, 2683 (2009).

[114] Alem, N., Erni, R. R., Kisielowski, C., Rossell, M. D., Gannett, W., and Zettl, A. Phys. Rev. B 80, 155425 (2009).

[115] Lee, C., Li, Q., Kalb, W., Liu, X., Berger, H., Carpick, R. W., and Hone, J. Science 328, 76 (2010).

[116] Otani, M. and Okada, S. Phys. Rev. B 83, 073405 (2011).

[117] Xiao, R., Tasnádi, F., Koepernik, K., Venderbos, J. W. F., Richter, M., and Taut, M. ARXIV-cond-mat.mtr-sci, 1105.4988v1 (2011).

[118] Castro, E. V., Novoselov, K. S., Morozov, S. V., Peres, N. M. R., dos Santos, J. M. B. L., Nilsson, J., Guinea, F., Geim, A. K., and Neto, A. H. C. Phys. Rev. Lett. 99, 216802 (2007).

[119] Shi, Y., Hamsem, C., Xia, X., Kim, K. K., Reina, A., Hofmann, M., Hsu, A. L., Zhang, K., li, H., Juang, Z.-Y., Dresselhaus, M. S., Li, L.-J., and Kong, J. Nano Lett. 10, 4134 (2010).

[120] Song, L., Ci, L., Lu, H., Sorokin, P. B., Jin, C., Ni, J., Kvashnin, A. G., Kvashnin, D. G., Lou, L., Yakobson, B. I., and Ajayan, P. M. Nano Lett. 10, 3209 (2010).

[121] Usachov, D., Adamchuk, V. K., Haberer, D., Grueneis, A., Sachdev, H., Preobrajenski, A. B., Laubschat, C., and Vyalikh, D. V. Phys. Rev. B 82, 075415 (2010).

[122] Artacho, E., Sánchez-Portal, D., Ordejón, P., García, A., and Soler, J. M. Phys. Status Solid B 215, 809 (1999).

[123] et al., J. M. S. J. Phys.: Condens. Matter 14, 2745 (2002).

[124] Troullier, N. and Martins, J. L. Phys. Rev. B 43, 1993 (1991).

[125] Junquera, J., Paz, ., Sánchez-Portal, D., and Artacho, E. Phys. Rev. B 64, 235111 (2001).

[126] Novoselov, K. S., Geim, A. K., Morozov, V. S., Jiang, D., Katsnelson, M. I., Grigoreva, V., Dubonos, S. V., and Firsov, A. A. Nature 438, 197 (2005). 
[127] Stankovich, S., Dikin, D. A., Dommett, G. H. B., Kohlhaas, K. M., Zimney, E. J., Stach, E. A., Piner, R. D., Nguyen, S. T., and Ruoff, R. S. Nature 442, 282 (2006).

[128] Fiori, G. and Iannaccone, G. IEEE Electron Device Lett. 28, 760 (2007).

[129] Liang, G., Neophytou, N., Lundstron, M. S., and Nikonov, D. E. J. Appl. Phys. 102, 054307 (2007).

[130] Son, Y. W., Cohen, M. L., and Louie, S. G. Nature 444, 347 (2006).

[131] Pisani, L., Chan, J. A., Montanari, B., and Harrison, N. M. Phys. Rev. B 75, 064418 (2007).

[132] Tapaszto, L., Dobrik, G., Lambin, P., and Biro, L. P. Nature Nanotechnology 3, 397 (2008).

[133] Soriano, D., Leconte, N., Ordejón, P., Charlier, J.-C., Palacios, J.-J., and Roche, S. Phys. Rev. Lett. 107, 016602 (2011).

[134] Soriano, D., Muñoz-Rojas, F., Fernández-Rossier, J., and Palacios, J. J. Phys. Rev. B 81, 165409 (2010).

[135] Rocha, A. R., Martins, T. B., Fazzio, A., and da Silva, A. J. R. Nanotechnology 21, 34 (2010).

[136] Martins, T. B., Miwa, R. H., da Silva, A. J. R., and Fazzio, A. Phys. Rev. Lett. 98, 196803 (2007).

[137] Ezawa, M. Phys. Rev. B 73, 045432 (2006).

[138] Brandbyge, M., Stokbro, K., Taylor, J., Mozos, J. L., and Ordejón, P. Mat. Res. Soc. Symp. Proc. 636, D9.25 (2000).

[139] di Ventra, M. Electrical Transport in Nanoscale Systems. Cambridge University Press, Cambridge, (2008).

[140] Martins, T. B., da Silva, A. J. R., Miwa, R. H., and Fazzio, A. Nano Lett. 8, 2293 (2008).

[141] Zheng, X. H., Rungger, I., Zeng, Z., and Sanvito, S. Phys. Rev. B 80, 235426 (2009). 
[142] Dutta, S., Manna, A. K., and Pati, S. K. Phys. Rev. Lett. 102, 096601 (2009).

[143] Mulder, W. A. J. Comp. Phys. 83, 303 (1989).

[144] Mittelmann, H. D. and Weber, H. SIAM J. Sci. Stat. Comput. 6, 49 (1985).

[145] Thole, C.-A. and Trottenberg, U. Appl. Math. Comp. 19, 333 (1986). 